Infections, including fracture-related infections and device-related infections, multiple biomate appor the treatment and prev orthopaedic infections are investigated. Enhanced antibiotic delivery by a bone targeting microparticle platform and a hydrogel delivery system for bacteriophages as alternative antimicrobials are introduced in this doctoral thesis. In order to reduce infection risk of orthopeadic devices, an anti-foulin and bactericidal coating was developed. These biomaterial approaches are shown to be efficient and feasible strategies for reducing the burden that infection places on our healthcare system.

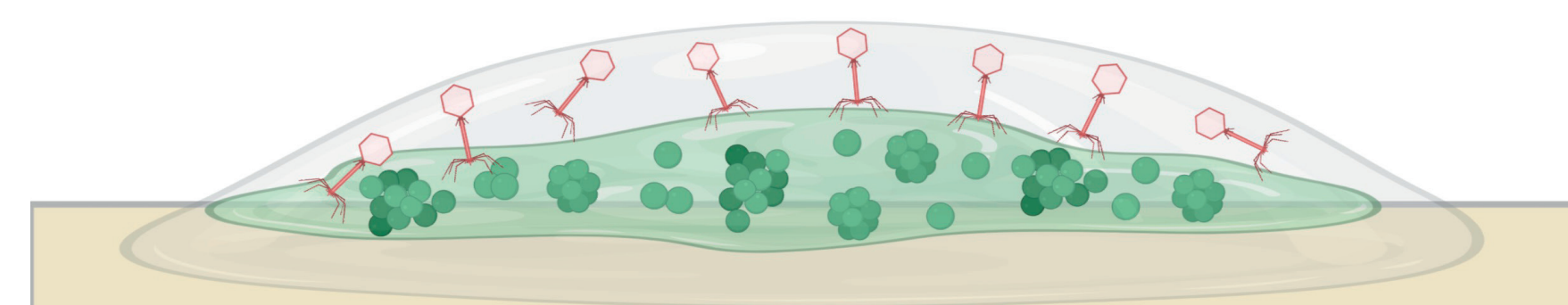

AO) unwessivor rwerre

\section{Invitation}

For attending the public defense of my $\mathrm{PhD}$ thesis
Biomaterial Approaches for the Treatment and Prevention of Orthopaedic Infections
Biomaterial Approaches for the Treatment and Prevention of Orthopaedic Infections

Stijn Gerard Rotman

on Thursday,

17th of December 2020

The defense can be followed live on the online platform of the University of Twente
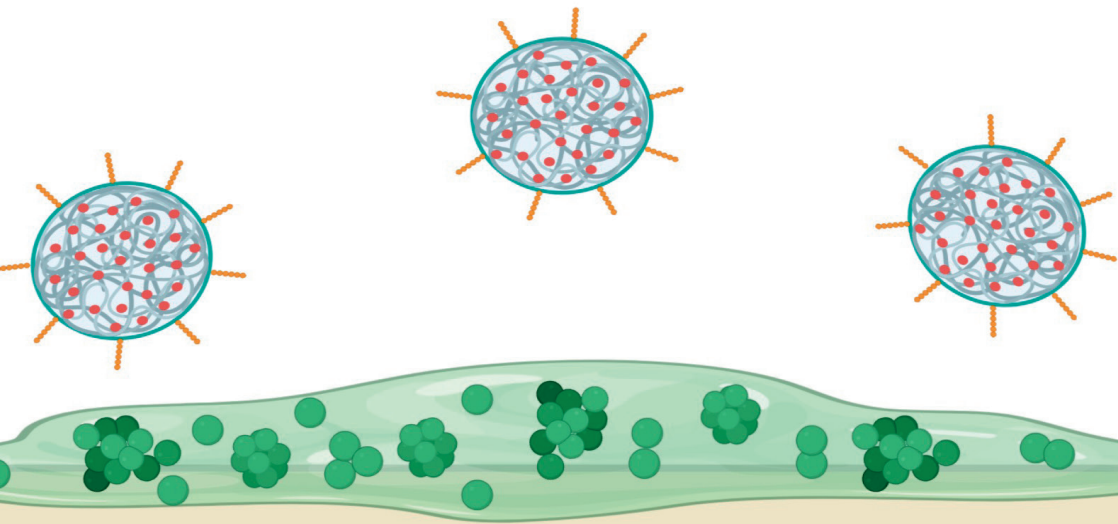

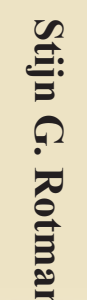




\section{BIOMATERIAL APPROACHES FOR THE TREATMENT AND PREVENTION OF ORTHOPAEDIC INFECTIONS}

Stijn Gerard Rotman 
Printed by: Ipskamp Printing, Enschede, the Netherlands

ISBN: 978-90-365-5092-5

DOI: $10.3990 / 1.9789036550925$

(C) 2020 Stijn Gerard Rotman, The Netherlands. All rights reserved. No parts of this thesis may be reproduced, stored in a retrieval system or transmitted in any form or by any means without permission of the author. Alle rechten voorbehouden. Niets uit deze uitgave mag worden vermenigvuldigd, in enige vorm of op enige wijze, zonder voorafgaande schriftelijke toestemming van de auteur. 


\title{
BIOMATERIAL APPROACHES FOR THE TREATMENT AND PREVENTION OF ORTHOPAEDIC INFECTIONS
}

\author{
DISSERTATION \\ to obtain \\ the degree of doctor at the Universiteit Twente, \\ on the authority of the rector magnificus, \\ Prof. dr. ir. A. Veldkamp, \\ on account of the decision of the Doctorate Board \\ to be publicly defended \\ on Thursday 17 December 2020 at 14.45 hours
}

by

Stijn Gerard Rotman

born on the 27th of July, 1992 in Hengelo, The Netherlands 
This dissertation has been approved by:

\author{
Supervisor \\ prof. dr. D.W. Grijpma \\ Co-supervisors \\ prof. dr. D.O.J. Eglin \\ dr. O. Guillaume
}




\section{Graduation Committee:}

Chair / secretary:

Supervisor:

Co-supervisors:

Committee Members: prof.dr. J.L. Herek

prof.dr. D.W. Grijpma

prof. dr. D.O.J. Eglin

dr. O. Guillaume

prof. dr. H.B.J. Karperien

prof. dr. J. Prakash

prof. dr. B. Nottelet

prof. dr. T. Tang

dr. F. Moriarty 



\section{Table of contents}

Chapter 1
Chapter 2

Chapter 3

Chapter 3 -

Appendix

Chapter 4

Chapter 5

Chapter 6

Chapter 7

Chapter 8

Chapter 9

Summary

Acknowledgements

General introduction

Drug delivery systems functionalized with bone mineral seeking agents for bone targeted therapeutics

Development of bone seeker-functionalized microspheres as a targeted local antibiotic delivery system for bone infections

Evaluation of bone targeting and antimicrobial properties of functionalized PCL microspheres in rat femoral defects

Bone targeted antibiotic delivery by poly(aspartic acid) functionalized poly $(\epsilon-$ caprolactone) microspheres

Antibiotic loaded PCL-PEG nanoparticles

Local bacteriophage delivery for treatment and prevention of bacterial infections thermoresponsive HA-pNIPAM hydrogels and alginate-chitosan microbeads as a treatment for infection

Synergistic anti-fouling and bactericidal poly(ether ether ketone) surfaces via a onestep photomodification

General conclusion and future perspectives 



\section{Chapter 1}

General introduction 


\section{General introduction}

Infection remains as one of the most frequent complications of orthopaedic fracture repair surgeries [1] and is associated with high socioeconomic burden and medical costs [2,3]. After internal fixation, closed bone fractures show a $1-2 \%$ infection rate while open bone fractures are much more susceptible to infection with incidence rates approaching 30\% [4,5]. In open bone defects, bacteria are in almost all cases introduced during or directly after the moment of trauma, making prophylactic antimicrobial treatment important in order to reduce the risk of onset of infection [6]. When prophylactic antimicrobials are not successful in preventing the onset of infection, acute and eventually chronic osteomyelitis (OM) develops which necessitates revision surgeries. Surgical revision includes the removal of infected implants and the debridement of surrounding contaminated tissues. The revision surgery is complemented with antibiotic regimens. It is generally accepted that both systemic and local antimicrobials are highly beneficial in treatment of OM, as systemic antibiotics have limited efficacy due to impaired vascularization at the bone fraction and implant site [5].

Local antibiotic medications have been implemented in orthopaedic surgeries for several decades and have improved outcome of infection treatments or lowered the incidence rate of OM [7]. Antibiotic loaded biomaterials (ALB) such as poly(methylmethacrylate) (PMMA) bone cements and collagen sponges have been implanted at the site of OM in order to establish a local antibiotic reservoir that releases antibiotics in a sustained manner [8]. The currently available ALB come with certain drawbacks such as incomplete or burst antibiotic release kinetics and, in the case of PMMA bone cements, non-biodegradability [9]. Incomplete drug 
release and non-biodegradability both require the ALB to be surgically extracted after antibiotic release is reduced to non-effective quantities. Release of left-over antibiotic below the minimal inhibitory concentration (MIC) contributes to the antibiotic resistances [10] and non-degraded ALB can act as a foreign body susceptible to bacterial colonization after the majority of antibiotic load has been released [11]. Additionally, the bulky dimensions of PMMA and collagen sponges also prevent close contact within the confinements of a complex bone fracture or debridement site. These shortcomings underline the necessity for the development of new ALB to improve patient care.

In the first chapters of the thesis, we hypothesized that an ALB with the ability to release a high dose of antibiotics at the interface of infected bone would improve the efficacy of local antibiotic therapies. Microspheres of poly( $\epsilon$-caprolactone) (PCL), a biodegradable polyester can be utilized as an antibiotic carrier and could be endowed with bone binding properties though surface incorporation of chelators such as Alendronate (ALN) or Aspartic acid oligomers (ASP). Such micron-sized ALB are able to penetrate small confinements at the fraction or debridement site and remain there due to their affinity to calcified tissues. With the release of antibiotic load at the interface of the contaminated bone tissue, a high local antibiotic concentration can be established, expected to exceed the MIC of bacteria frequently associated with OM.

As resistances to antibiotics are a growing concern, there are benefits in the development of delivery systems for alternative antimicrobials. Therefore, the second part of this dissertation focusses on local delivery of bacteriophages (phages) at the site of OM. Phages are viruses that infect and 
lyse their host bacteria, while simultaneously undergoing a self-amplifying cycle, resulting in an amplified local phage presence. Without host bacteria present, the phage virus would decay or be cleared by phagocytosis and cytokine response of the patient's immune system [12]. This makes bacteriophages, to a certain degree, a self-dosing antimicrobial. The advantages of such a self-regulating antimicrobial virus are evident, but phage amplification is impaired when insufficient bacterial host is present. Together with the adverse interactions of phages with the immune system, successful therapy generally requires either repeated phage administrations or sustained phage release from a biomaterial phage carrier to achieve long term antimicrobial activity [13]. In contrast to the solid PCL microspheres as antibiotic carriers, our approach towards phage delivery consists of a hyaluronic acid-poly( $N$-isopropylacrylamide) (HA-pNIPAM) copolymer hydrogel. Hydrogels are considered highly suitable phage carriers due to their tailorability and water-rich environment which limits adverse matrix-phage interactions. Additionally, HA-pNIPAM hydrogels show thermo-responsive behavior by undergoing a semi-rapid sol-gel transition at approximately $30^{\circ} \mathrm{C}$, allowing the gels to initially flow into the confinements of the bone fracture or debridement site after which gelation occurs, fixing the phage loaded gel in place.

While antibiotic or phage delivery systems can be implemented for both infection treatment and prevention, the development of antimicrobial implants is an additional method of reducing bacterial burdens in orthopedic surgery. Orthopaedic implants are commonly used to fill bone defects, act as fracture support structures or restore function to load bearing joints and are susceptible to bacterial colonization. This is because the patient's immune 
response [14] and effects of systemic drugs are severely diminished at the surface of the implant [5].

Polyether ether ketone (PEEK) is a polymer frequently used in orthopaedic surgery as an implant material to fill bone defects or as a fixation device [15]. Aiming to reduce bacterial adhesion to such medical implants, technologies such as bioactive nano-topographies (e.g. nano-pillars) [16] and anti-fouling surfaces [17] have emerged. However, conflicting reports indicate that surface roughness assists in bacterial adhesion and biofilm formation [18,19], resulting in the anti-fouling functionalization approach being the investigated strategy in this work. While anti-fouling surfaces are often only reducing or slowing down bacterial adhesion, an active bactericidal surface feature is desired to prevent the onset of bacterial colonization and subsequent biofilm formation on the implant. Coatings of cationic polymers as chitosan derivatives [20] or polyethyleneimine [21] have exhibited antimicrobial properties against a wide variety of bacterial strains. Combined coatings of anti-fouling polymers and cationic bactericidal polymers is expected to synergistically reduce bacterial contamination of PEEK implants.

\section{Aim of the dissertation}

The aim of the research presented in this dissertation is to develop biomaterials to treat or to prevent the onset of orthopaedic infections. Several approaches have been investigated, including a bone targeting antibiotic delivery system and a thermo-responsive hydrogel delivery system for bacteriophages. To prevent the onset of implant related orthopaedic infections, an anti-fouling and bactericidal photo-inserted coating for PEEK implants has been developed and characterized. In the following outline, the topics of the individual chapters are listed. 


\section{Outline of the dissertation}

Chapter 2 consists of an introduction to bone targeted drug delivery. Biological targets in the bone are identified. Different bone targeting groups and the drug delivery systems in which they are incorporated are reviewed.

In Chapter 3 a PCL microsphere system with hydrophobic gentamicin (GM-AOT) load is introduced. Surface-functionalization with Alendronate (ALN) was performed to give the drug delivery system affinity to calcified tissues. The effect of surface grafted ALN on osteoclasts was assessed, identifying the advantages of other bone targeting moieties.

Chapter 3 - Appendix shows in vitro bone binding assays in a rat femoral defect model with PCL-ALN and PCL-ASP microspheres and investigates antimicrobial properties of GM-AOT loaded microspheres.

Chapter 4 shows improvements made on the drug delivery system from chapter 3, replacing Alendronate with aspartic acid oligomers and introducing a different functionalization strategy so that harsh $\mathrm{NaOH}$ surface treatment can be omitted.

Chapter 5 introduces and characterizes PCL-PEG nanoparticles for GMAOT delivery.

Chapter 6 introduces bacteriophages as antimicrobials that can be an attractive alternative to antibiotics. The potential of local bacteriophage delivery by carrier systems to treat infections is revealed and compatible processing methods are discussed. Literature related to local bacteriophage delivery with a wide range of biomaterials is outlined. 
In Chapter 7 a HA-pNIPAM hydrogel facilitated for bacteriophage delivery is presented. Addition of Alginate/chitosan beads incorporated into the hydrogel are investigated for their tailored release kinetics of the phage load.

Chapter $\mathbf{8}$ focusses on a post fabrication functionalization of antifouling and bactericidal polymers on the surface of PEEK implants. PEEK implants endowed with anti-fouling and bactericidal polymers are physio-chemically characterized and their in vitro anti-fouling and bactericidal properties are demonstrated. An infectious subdermal in vivo murine model is introduced, and the functional PEEK implants were investigated.

In Chapter 9 the work presented in this thesis is subjected to a general discussion and future perspectives in orthopedic infection prophylaxis or treatment are considered.

\section{References}

1 Leonardo, F., Domenico, F. \& Michele Attilio, R. Surgical Site Infection In Orthopaedic Surgery: Correlation Between Age, Diabetes, Smoke And Surgical Risk. Folia Medica 56, 259-263, doi:https://doi.org/10.1515/folmed-2015-0005 (2014).

2 Poultsides, L., Liaropoulos, L. \& Malizos, K. The socioeconomic impact of musculoskeletal infections. J Bone Joint Surg Am 92, e13, doi:10.2106/JBJS.I.01131 (2010).

3 Ankit, B., Gupta, S. \& Noble, B. Oral Sustained Release Dosage Form: An Opporturnity to Prolong the Release of Drug. IJARPB (2013).

4 Trampuz, A. \& Zimmerli, W. Diagnosis and treatment of infections associated with fracture-fixation devices. Injury 37 Suppl 2, S59-66, doi:10.1016/j.injury.2006.04.010 (2006).

5 Metsemakers, W. J. et al. Infection after fracture fixation: Current surgical and microbiological concepts. Injury 49, 511-522, doi:10.1016/j.injury.2016.09.019 (2018).

6 Chang, Y. et al. Antibiotic Prophylaxis in the Management of Open Fractures: A Systematic Survey of Current Practice and Recommendations. JBJS Reviews 7, e1, doi:10.2106/jbjs.rvw.17.00197 (2019).

7 van Vugt, T. A. G., Arts, J. J. \& Geurts, J. A. P. Antibiotic-Loaded Polymethylmethacrylate Beads and Spacers in Treatment of Orthopedic Infections 
and the Role of Biofilm Formation. Front Microbiol 10, 1626-1626, doi:10.3389/fmicb.2019.01626 (2019).

8 ter Boo, G.-J. A., Grijpma, D. W., Moriarty, T. F., Richards, R. G. \& Eglin, D. Antimicrobial delivery systems for local infection prophylaxis in orthopedic- and trauma surgery. Biomaterials doi:https://doi.org/10.1016/j.biomaterials.2015.02.020 (2015).

9 Kluin, O. S., van der Mei, H. C., Busscher, H. J. \& Neut, D. Biodegradable vs nonbiodegradable antibiotic delivery devices in the treatment of osteomyelitis. Expert Opinion on Drug Delivery 10, 341-351, doi:10.1517/17425247.2013.751371 (2013).

10 Walenkamp, G. H., Vree, T. B. \& van Rens, T. J. Gentamicin-PMMA beads. Pharmacokinetic and nephrotoxicological study. Clinical orthopaedics and related research, 171-183 (1986).

11 Bertazzoni Minelli, E., Della Bora, T. \& Benini, A. Different microbial biofilm formation on polymethylmethacrylate (PMMA) bone cement loaded with gentamicin and vancomycin. Anaerobe 17, 380-383, doi:https://doi.org/10.1016/j.anaerobe.2011.03.013 (2011).

12 Van Belleghem, J. D., Dąbrowska, K., Vaneechoutte, M., Barr, J. J. \& Bollyky, P. L. Interactions between Bacteriophage, Bacteria, and the Mammalian Immune System. Viruses 11, 10, doi:10.3390/v11010010 (2018).

13 Malik, D. J. et al. Formulation, stabilisation and encapsulation of bacteriophage for phage therapy. Advances in Colloid and Interface Science 249, 100-133, doi:https://doi.org/10.1016/j.cis.2017.05.014 (2017).

14 Rochford, E. T. J., Richards, R. G. \& Moriarty, T. F. Influence of material on the development of device-associated infections. Clinical Microbiology and Infection 18, 1162-1167, doi:https://doi.org/10.1111/j.1469-0691.2012.04002.x (2012).

15 Kurtz, S. M. \& Devine, J. N. PEEK biomaterials in trauma, orthopedic, and spinal implants. Biomaterials 28, 4845-4869, doi:10.1016/j.biomaterials.2007.07.013 (2007).

16 Liu, W. et al. Bioinspired polyethylene terephthalate nanocone arrays with underwater superoleophobicity and anti-bioadhesion properties. Nanoscale 6 , 13845-13853, doi:10.1039/C4NR04471A (2014).

17 Lowe, S., O'Brien-Simpson, N. M. \& Connal, L. A. Antibiofouling polymer interfaces: poly(ethylene glycol) and other promising candidates. Polymer Chemistry 6, 198-212, doi:10.1039/C4PY01356E (2015).

18 Teughels, W., Van Assche, N., Sliepen, I. \& Quirynen, M. Effect of material characteristics and/or surface topography on biofilm development. Clinical oral implants research 17 Suppl 2, 68-81, doi:10.1111/j.1600-0501.2006.01353.x (2006).

19 Dantas, L. C. d. M. et al. Bacterial Adhesion and Surface Roughness for Different Clinical Techniques for Acrylic Polymethyl Methacrylate. Int J Dent 2016, 86857968685796, doi:10.1155/2016/8685796 (2016).

20 Pranantyo, D., Xu, L. Q., Kang, E.-T. \& Chan-Park, M. B. Chitosan-Based Peptidopolysaccharides as Cationic Antimicrobial Agents and Antibacterial Coatings. Biomacromolecules 19, 2156-2165, doi:10.1021/acs.biomac.8b00270 (2018).

21 Gibney, K. A. et al. Poly(ethylene imine)s as antimicrobial agents with selective activity. Macromol Biosci 12, 1279-1289, doi:10.1002/mabi.201200052 (2012). 


\section{Chapter 2}

\section{Drug delivery systems functionalized with bone mineral seeking agents for bone targeted therapeutics}

S.G. Rotman ${ }^{1,2}$, D.W. Grijpma ${ }^{2}$, R.G. Richards ${ }^{1}$, T.F. Moriarty ${ }^{1}$, D. Eglin ${ }^{1}$ and O. Guillaume ${ }^{1}$

${ }^{1}$ AO Research Institute Davos, Davos Platz, Switzerland

${ }^{2}$ Department of Biomaterials Science and Technology, Faculty of Science and Technology, University of Twente, Enschede, The Netherlands

Published in Journal of Controlled Release 269 (2018) 88-99 


\section{Abstract}

The systemic administration of drugs with a target site of action in the bone is often associated with poor uptake of the drug in the targeted tissue, potential systemic toxicity, and suboptimal efficacy. To overcome these limitations, many micro- and nano-sized drug carriers have been developed for the treatment of bone pathologies that exhibit specific affinity for bone. Drug carriers can be functionalized with bone mineral seekers (BMS), creating a targeted drug delivery system (DDS) which is able to bind to bone and release therapeutics directly at the site of interest. This class of advanced DDS is of tremendous interest due to their strong affinity to bone, with great expectation to treat life-threatening bone disorders such as osteomyelitis, osteosarcoma or even osteoporosis. In this review, we first explain the mechanisms behind the affinity of several well-known BMS to bone, and then we present several effective approaches allowing the incorporation BMS into advanced DDS. Finally, we report the therapeutic applications of BMS based DDS under development or already established. Understanding the mechanisms behind the biological activity of recently developed BMS and their integration into advanced therapeutic delivery systems are essential prerequisites for further development of bone-targeting therapies with optimal efficacy.

\section{Introduction}

For any drug to achieve its optimal therapeutic effect, it is important that the compound reaches, and is retained, at the intended site of action (tissue, receptor, or molecules) without losing its chemical integrity or biological function. The most frequently applied method to deliver drugs has 
traditionally been systemic administration. However, this is associated with certain drawbacks, most important being the limited penetration of drugs to their sites of action and the associated systemic side effects of the resulting high dosages. Drug delivery systems (DDS) have emerged to improve drug concentrations in tissues while preventing structural changes of the incorporated drugs. In addition, DDS offer the possibilities to increase the range of applications of hydrophobic compounds (by enhancing their solubility [1]), prolong efficacy of drugs with short biological half-life (by sustained drug release mechanisms [2]), and limit non-specific cellular uptake of drugs (by reducing opsonization by macrophages) [3]. However, conventional DDS that increase biological circulation time are not necessarily designed to actively reach, penetrate, and concentrate at the intended site of action. Targeting strategies that can be used by DDS include not only exploitation of the passive enhanced permeability and retention (EPR) effect, but also active binding to specific tissues when combined with biologically affine moieties [4].

Systemic DDS are usually nanoscale constructs that can be injected intravenously, administered orally or even can be introduced in vivo by pulmonary inhalation. Their small size allows them to reach even the smallest capillaries and the limited clearance of such nanoscale constructs from the blood by macrophages gives them stealth-like properties, resulting in longer circulation times.

In the orthopedic field, bone related diseases such as osteoporosis, osteosarcoma and osteomyelitis are regularly treated via conventional systemic drug administrations. Nevertheless, inefficient uptake of drugs by 
bone can limit the utility of these drugs or severely compromise treatment outcome.

For example, bone infections are routinely treated with systemically administered antibiotic agents, for extended periods of time. However, penetration of antibiotics into the affected bone compartment has been reported to be inefficient, with low local drug concentration at the site of infection [5], which can further increase the risk of the development of drugresistant infections [6]. Additionally, the prolonged antibiotic regimens required to successfully treat these infections raise healthcare costs and can lead to toxic hepatic side effects and nephrotoxicity [6]. In consequence, systemic antibiotherapies are regularly combined with DDS applied locally in infected bones or bone fractures. The most commonly used local DDS used in these circumstances are antibiotic-loaded bead cements of poly(methyl methacrylate) (PMMA) [7]. Limitations of PMMA implants include a lack of biodegradable properties, the need for invasive implantation and retrieval surgeries and an incomplete release of the loaded antibiotic [8].

In order to establish a high and sustained local concentration of a drug in the proximity of bone, it is desired to have a DDS which can interact intimately with bone tissue on a physical and chemical level. There is a wide array of molecules available that have affinity to bone tissue, called bone mineral seekers (BMS). These compounds can be implemented in a DDS design and would result in DDS exhibiting preferential affinity at bony sites where they can locally release their drug load. This would then lead to high drug concentrations at the therapeutic target site and to a better efficacy of the treatment. 
The aim of this review is to provide an overview regarding the features of recent DDS that actively target bone tissue via the utilization of bone seekers. Then, in a second part, we report the different fabrication strategies and the bone targeting efficiency of BMS-based DDS. The review concludes with an overview of some of the most promising pre-clinical and clinically applied DDS making successful use of BMS.

\section{Composition of bone: possible biological targets for bone- seeking agents}

The organic matrix of bone represents roughly $30 \%$ of total dry bone mass (see Table 1). This organic matrix includes $90 \mathrm{wt} \%$ collagen fibrils in dry weight. The remaining components consist of glycoproteins, proteoglycans and other proteins [9]. The inorganic matrix (65-70\% of dry bone mass) consists of calcium-deficient hydroxyapatite (dHAP) nanocrystals which are embedded in the organic matrix $[9,10]$. Bone cells represent only $1-2 \%$ of the total dry bone mass and mostly consist of osteocytes present in the bone matrix. Osteoblast and osteoclast cells regulate bone homeostasis by promoting the synthesis of bone matrix or resorbing bone matrix respectively. Harversian and Volkmann's channels provide space for blood vessels to transport nutrients and oxygen to the organic bone components. These channels of approximately $70 \mu \mathrm{m}$ of diameter provide accessibility to bone tissue for therapeutic agents [9]. 
Table 2.1 - Overview of bone components, their approximate dry weight percentage in healthy bone and examples of potential targeting moieties for DDS.

\begin{tabular}{|c|c|c|c|}
\hline \multicolumn{2}{|c|}{ Bone component } & \multirow{2}{*}{$\begin{array}{c}\begin{array}{c}\text { Presence in } \\
\text { bone }\end{array} \\
\text { (dry weight) } \\
27-32 \%\end{array}$} & \multirow{2}{*}{$\begin{array}{l}\begin{array}{l}\text { Targeting } \\
\text { moieties }\end{array} \\
\text { Fibronectin, } \\
\text { Entactin }\end{array}$} \\
\hline \multirow[t]{2}{*}{$\begin{array}{l}\text { Organic } \\
\text { matrix }\end{array}$} & Collagen (type I) & & \\
\hline & $\begin{array}{l}\text { Non-collagenous } \\
\text { proteins (e.g. } \\
\text { Osteocalcin, bone } \\
\text { morphogenetic } \\
\text { proteins (BMPs) and } \\
\text { fibronectin) }\end{array}$ & $<1 \%$ & $\begin{array}{l}\text { Multitude of } \\
\text { commercially } \\
\text { available protein } \\
\text { specific antibodies } \\
\text { and their } \\
\text { fluorophore } \\
\text { conjugates }\end{array}$ \\
\hline $\begin{array}{l}\text { Cellular } \\
\text { content }\end{array}$ & $\begin{array}{l}\text { Osteocytes, } \\
\text { osteoblasts and } \\
\text { osteoclasts }\end{array}$ & $1-2 \%$ & $\begin{array}{l}\text { DMP1, sclerostin, } \\
\text { MEPE }\end{array}$ \\
\hline $\begin{array}{l}\text { Inorganic } \\
\text { matrix }\end{array}$ & $\begin{array}{l}\text { Ca-deficient } \\
\text { Hydroxyapatite }\end{array}$ & $65-70 \%$ & $\begin{array}{l}\text { Bisphosphonates, } \\
\text { Tetracyclines, } \\
\text { Poly (aspartic acid), } \\
\text { Poly(glutamic acid) }\end{array}$ \\
\hline
\end{tabular}

DMP1: Dentin matrix acidic phosphoprotein 1, MEPE: Matrix extracellular phosphoglycoprotein

Being the major organic macromolecule present in the bone, collagen could make an attractive target for bone seeker modified DDS. Fibronectin, entactin as well as some glycoproteins have been reported to bind with high affinity to collagen [11]. However, collagen is also the body's most abundant protein [12], highly present in cartilage and connective tissues, meaning that a DDS with specific affinity to collagen will be extremely non-specific to bone tissue. Organic matrix proteins (i.e. osteocalcin, osteonectin and osteopontin) could be targeted by a broad range of antibodies [13]. Even though such antibodies would display a high specificity, the relatively low 
amount of these bone-associated proteins $(<1 \%$ of total dry bone mass) could compromise the effectiveness of such binding strategy. Osteocytes could potentially represent a very specific target for bone tissue. Some osteocyte markers like dentin matrix protein 1 (DMP1), sclerostin and matrix extracellular phosphoglycoprotein (MEPE) are reported in literature and could be used in targeting these cells [14]. Nevertheless, bone cells do not represent attractive targets for DDS as they are embedded in dense inorganic matrix, making them poorly accessible for DDS constructs. The inorganic matrix of bone, consisting nearly entirely of dHAP, is the major component of bone tissue and it offers an excellent target for BMS functionalized DDS due to its exclusive location in bones and developing teeth.

\section{Bone affinity of bone mineral seeking agents}

There are many different types of BMS, ranging from small molecules (< $1000 \mathrm{Da}$ ) to large macromolecular proteins. When considering the use of such molecules or macromolecules for the functionalization of a DDS, several factors are essential for a successful targeted DDS [15,16]. First, the BMS units should have great affinity toward bone mineral and its incorporation in a DDS should not impair its ability to interact with dHAP. Secondly, the BMS-DDS construct should neither trigger any toxic or adverse side effects, nor interfere with healthy bone homeostasis upon administration. Finally, the DDS must not hinder the therapeutic capacities of the delivered drug. Depicted in Table 2 are compounds belonging to the different classes of BMS that are discussed throughout this review, with a summary of their main advantages and disadvantages. 
Table 2.2 - Overview of the main classes of BMS and their advantages and disadvantages for utilization in bone seeking DDS. General structure of Bisphosphonates (A), the multi-phosphonate containing molecule EDTMP (B), Tetracycline (C) and the bone seeking peptide poly ( $\alpha$-D-aspartic acid) (D).

A<smiles>[R2]C([R2])(P(=O)([O-])[O-])P(=O)([O-])[O-]</smiles>

Generic BP structure

C

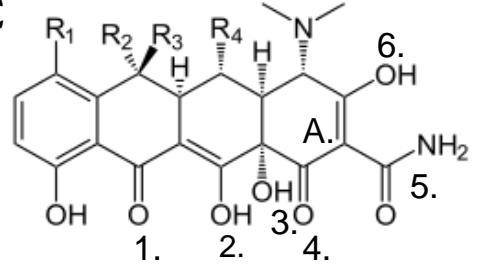

Tetracycline
B<smiles>O=P(O)(O)CN(CCN(CP(=O)(O)O)CP(=O)(O)O)CP(=O)(O)O</smiles>

EDTMP

D<smiles>CC(C)(C)NC(=O)[C@H](CC(=O)O)C(C)(C)C</smiles>

poly $(\alpha-\mathrm{D}$-aspartic acid $)$

\begin{tabular}{|c|c|c|}
\hline Group & Advantages & Disadvantages \\
\hline $\begin{array}{l}\text { A. } \\
\text { Bisphosphonates }\end{array}$ & $\begin{array}{l}\text { - Strong and rapid affinity to bone } \\
\text { mineral } \\
\text { - Easily conjugated into DDS by } \\
\text { using } \mathrm{R}_{1} \text { and } \mathrm{R}_{2} \text { side chains }\end{array}$ & $\begin{array}{l}\text { - Potency to inhibit } \\
\text { osteoclasts and bone } \\
\text { homeostasis } \\
\text { - Very long presence at bone } \\
\text { sites }\end{array}$ \\
\hline $\begin{array}{l}\text { B. Multi- } \\
\text { phosphonate- } \\
\text { containing } \\
\text { molecules }\end{array}$ & $\begin{array}{l}\text { Level of bone affinity is scalable } \\
\text { with incorporation of more or } \\
\text { less phosphonate groups } \\
\text { - Ability to facilitate the transport } \\
\text { of radiopharmaceuticals }\end{array}$ & $\begin{array}{l}\text { - Suboptimal distance } \\
\text { between oxygen groups } \\
\text { involved in chelation to } \\
\text { bone }\end{array}$ \\
\hline C. Tetracyclines & $\begin{array}{l}\text { - High affinity and specificity to } \\
\text { developing bone sites } \\
\text { - Intrinsic antibiotic and bone } \\
\text { targeting properties }\end{array}$ & $\begin{array}{l}\text { - Low affinity to bone sites } \\
\text { with low bone turn-over } \\
\text { - Stains developing teeth and } \\
\text { impairs bone development }\end{array}$ \\
\hline $\begin{array}{l}\text { D. Bone affine } \\
\text { peptides }\end{array}$ & $\begin{array}{l}\text { - Biodegradable properties allow } \\
\text { the clearance of the DDS within } \\
\text { the therapeutic timeframe } \\
\text { - Highly tunable bone affinity due } \\
\text { to custom peptide synthesis }\end{array}$ & $\begin{array}{l}\text { - Peptide bonds linking } \\
\text { individual amino acids are } \\
\text { prone to hydrolysis before } \\
\text { target site is reached }\end{array}$ \\
\hline
\end{tabular}

EDTMP: ethylenediamine tetra (methylene phosphonic acid) 
Comparative studies of the mineral affinities of different classes of BMS are rare, and bone affinity is often reported in relation to control groups (usually non-targeting DDS analogues), which hinders any absolute assessment in terms of bone affinity of different classes of BMS. Among the limited literature available, the reports by Ross et al. included comparative studies for bisphosphonate-, L-glutamic acid- and 2-amino-ethylphosphonic acidfunctionalized gold nanoparticles (Au-NP), and their interaction to dHAP crystals [17] and bone [18]. Among those Au-NP delivery systems, bisphosphonates showed the highest affinity to dHAP and bone.

\section{Bisphosphonates}

Bisphosphonate (BP) molecules have been studied extensively since the 1960's [19] and a multitude of BP-based products are commercially available. BPs contain two phosphonate groups $\left(\mathrm{PO}\left(\mathrm{O}^{-}\right)_{2}\right)$ sharing a common carbon atom, also known as a P-C-P backbone (a generic BP is depicted in Table 2, compound A). BPs are the analogues of naturally occurring pyrophosphate, which is an anhydride and a regulator of bone mineralization, characterized by its P-O-P bond. The nature of this bond makes pyrophosphates prone to fast enzymatic hydrolysis as part of the normal bone physiology and are therefore not suitable as a therapeutic agent or BMS [10]. The P-C-P backbone of BPs is far more stable, while preserving its affinity to bone mineral. Thus, BPs exhibit a prolonged residence in the bone tissue, up to many years [20]. These properties (along with their action on osteoclast inhibition) are the reason for the utilization of BPs as bone antiresorptive drugs in osteoporotic patients. Mechanistically, BPs exhibit affinity toward bone by chelating with divalent calcium ions $\left(\mathrm{Ca}^{2+}\right)$ present in dHAP. The deprotonated hydroxyl ( $\left(\mathrm{P}_{-} \mathrm{O}^{-}\right)$of the two phosphonates in BPs are separated 
approximately by 2.9 to $3.1 \AA$. This is similar to the distance in $\mathrm{Ca}^{2+}$-chelating oxygen atoms naturally present in dHAP crystals, leading to competitive affinity to $\mathrm{Ca}^{2+}$ ions [21]. In BPs, the distance between the two deprotonated hydroxyl groups increases when the P-C-P bond is replaced with P-N-P or P-C-C-P bonds, leading to reduced affinity toward dHAP of such compounds [22]. The two remaining groups on the P-C-P carbon atom, $\mathrm{R}_{1}$ and $\mathrm{R}_{2}$, can further modulate affinity to dHAP. For instance, the presence of a hydroxyl or an amine group at $\mathrm{R}_{1}$ leads to additional interaction with the calcium ions and these BPs indeed show a higher affinity toward dHAP compared to other BPs [23,24]. Changing the $\mathrm{R}_{2}$ group with moieties containing nitrogen atoms leads to a significant change in osteogenic antiresorption potency, making those BPs not only suitable as bone seekers for DDS but also potent anti-osteoporotic drugs [24,25]. Nitrogen containing BPs inhibit the synthesis of farnesyl pyrophosphate, which controls osteoclast activity [26]. Reduced osteoclast activity shifts the bone homeostasis toward bone formation as osteoblast bone formation remains unaffected. With the affinity of those BPs toward calcium mineral and farnesyl pyrophosphate synthase being highly specific, they preferentially accumulate in bone tissue. Nancollas et al. conducted analysis of 6 different BPs commonly used in clinics [24], and presented BPs ranking as followed on in vitro dHAP affinity: Chlodronate $<<$ Etidronate $<$ Risedronate $<$ Ibandronate $<$ Alendronate $<$ Zoledronate (Table 3). 
Table 2.3 - Overview of common BPs with their constitutive side chains. BPs are ranked by potency toward osteoclast inhibition relative to etidronate (due to the presence of nitrogen $(N)$ in the $R^{2}$ chain), determined by dHAP crystal growth rates analysis [26]. The kinetic affinity constant $\left(\mathrm{K}_{\mathrm{L}}\right)$ is an indication of the measure of affinity between dHAP and the different BPs [24].

\begin{tabular}{|c|c|c|c|c|c|}
\hline Class & Compound & $\begin{array}{l}\text { Osteoclast } \\
\text { inhibition } \\
\text { potency } \\
{[26]}\end{array}$ & 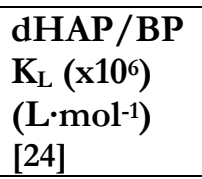 & $\mathbf{R}_{1}$ & $\mathbf{R}_{2}$ \\
\hline \multirow{2}{*}{$\begin{array}{l}\text { Non-N } \\
\text { containin } \\
\text { g BPs }\end{array}$} & Etidronate & $1 \mathrm{x}$ & 1.19 & $\overline{\mathrm{OH}}$ & $-\mathrm{CH}_{3}$ \\
\hline & Chlodronate & $10 \mathrm{x}$ & 0.72 & $-\mathrm{Cl}$ & $-\mathrm{Cl}$ \\
\hline \multirow{4}{*}{$\begin{array}{l}\mathrm{N}- \\
\text { containin } \\
\mathrm{g} \mathrm{BPs}\end{array}$} & Alendronate & $500 x$ & 2.94 & $\overline{-}-\overline{\mathrm{OH}}$ & $-\left(\mathrm{CH}_{2}\right)_{3}-\mathrm{NH}_{2}$ \\
\hline & Ibandronate & $1000 x$ & 2.36 & - & $\begin{array}{l}-\left(\mathrm{CH}_{2}\right)_{2-} \\
\mathrm{N}\left(\mathrm{CH}_{3}\right)^{-} \\
\left(\mathrm{CH}_{2}\right)_{4}{ }^{-} \mathrm{CH}_{3}\end{array}$ \\
\hline & Risendronate & $2000 x$ & 2.19 & - & $\begin{array}{l}-\mathrm{CH}_{2}-\left(\mathrm{NC}_{5} \mathrm{H}_{4}\right) \\
\text { (ring) }\end{array}$ \\
\hline & Zoledronate & $10000 x$ & 3.47 & $\overline{\mathrm{OH}}$ & $\begin{array}{l}-\mathrm{CH}_{2^{-}} \\
\left(\mathrm{N}_{2} \mathrm{C}_{3} \mathrm{H}_{3}\right) \\
\text { (ring) }\end{array}$ \\
\hline
\end{tabular}

The involvement of phosphonate groups in the dHAP binding mechanism was further evaluated by Puljula et al. [27], who investigated the effect of phospho-esters on the ability of the BP to bind to calcium sufficient dHAP. The BPs with low affinity to bone (e.g. Chlodronate) was not able to bind to bone when one of the four chelating oxygen groups was used to form methoxy esters or phenol esters. The more potent BPs with hydroxyl groups on the $\mathrm{R}_{1}$ side chain were able to chelate with $\mathrm{dHAP}$ after the esterification of two oxygen groups, but in significantly reduced quantity compared to their non-modified analogues [27]. This research emphasizes the fact that the hydroxyl $\mathrm{R}_{1}$ group is involved in $\mathrm{dHAP}$ binding and that the amount of $\mathrm{BP}$ esterification is negatively correlated to the ability of the BP to bind to dHAP. 
Importantly for the bone seeking DSS, the hydroxyl- and amine groups positioned at $\mathrm{R}_{1}$ and $\mathrm{R}_{2}$ can be used for chemical conjugation with a drug (to create a prodrug conjugate) [28] or to the surface of a particulate polymer carrier [29] without altering affinity to bone. Interestingly, it is not reported to our knowledge, if using the nitrogen $\mathrm{R}_{2}$ group for conjugation could decrease the binding affinity of BPs to farnesyl pyrophosphate synthetase and have an impact on its ability to reduce bone mineral resorption. It could be hypothesized that Alendronate tethered at the $\mathrm{R}_{2}$ position to DDS should exhibit a strong affinity to mineral but with a reduced osteoclast inhibition (so reduced potential side effects), but this has still to be demonstrated.

Nevertheless, it must be emphasized that bisphosphonates are able to display some side effects. A study by Brown et al. listed several potential complications that could be associated with long term ( $>5$ years) bisphosphonate administration [30]. Bisphosphonate related osteonecrosis of the jaw (BRONJ), atypical sub-trochanteric fractures in the femur and esophageal cancer are some of the reported secondary effects. However, most of these complications are reported in small studies or clinical cases and it remains difficult to establish causative evidence. It is recognized that BP treatment becomes a significant risk factor for the development of BRONJ after invasive dental procedures, like teeth extractions, with incidence up to $27.5 \%$ reported after 1 to 4 years of Zoledronate treatment [31]. It is worth pointing out that these risk assessment studies of BPs [30,31] have been carried out to evaluate the side effects of systemic administration of BPs as a stand-alone therapy over a prolonged duration of administration. When BPs are incorporated in local DDS, the BP associated side effects like BRONJ might be reduced due to the negligible systemic diffusion of the BP and the relatively short duration of the therapy (perhaps even single 
administration). For comparison, typical dosages of BP therapy for osteoporosis treatment are in the range of 5 to $70 \mathrm{mg}$ per week [32] while a typical BP functionalized DDS would only expose the patient to $\pm 1 \mathrm{mg}$ of Alendronate for $400 \mathrm{mg}$ of DDS construct [33].

\section{Other Phosphonate-containing molecules}

BPs are not the only type of molecules with phosphonate groups that exhibit affinity to dHAP. Ethylenediamine tetra(methylene phosphonic acid) (EDTMP, Table 2 compound B) and tetraazacyclotetradecane-1,4,8,11tetramethylene phosphonic acid (DOTMP), both with 4 phosphonate groups, are known to chelate to $\mathrm{Ca}^{2+}$ ions and have primarily been used to transport radiopharmaceuticals [34] and also proteins to bone [35]. In contrast to BPs, no physiological effects of such phosphonate containing molecules on bone homeostasis have been reported.

To increase the amount of phosphonate groups available to chelate $\mathrm{Ca}^{2+}$ ions, multiple BPs can be associated together to form dendritic structure, using the $\mathrm{R}_{2}$ group of the $\mathrm{BP}$ and a spacer (e.g. 3,5-di(ethylamino-2,2bisphosphono)benzoic acid) to create prodrug branched structures [35]. Bansal et al. prepared compounds with incorporated bisphosphonate groups and covalently attached bovine serum albumin or nonspecific bovine immunoglobulin-G as model drugs (Figure 2.1A). Mineral affinity was significantly enhanced (compared to non-modified proteins, Figure 2.1B), and was proportional to the number of BP moieties (Figure 2.1C) [35]. 


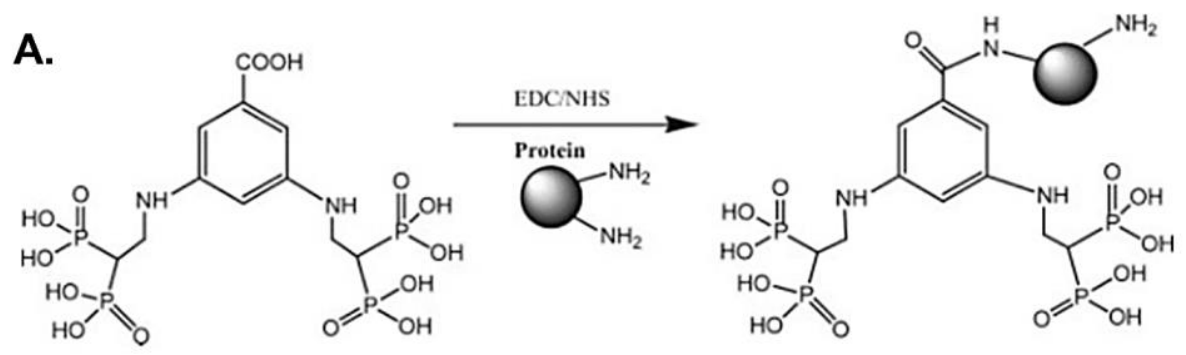

B.

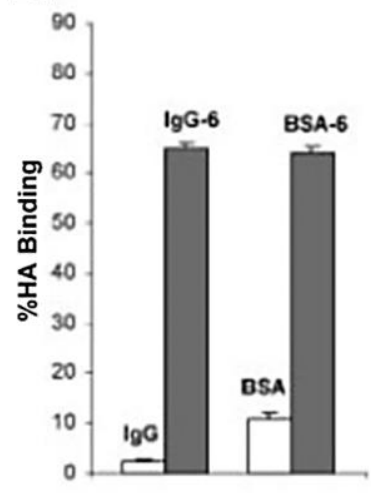

C.

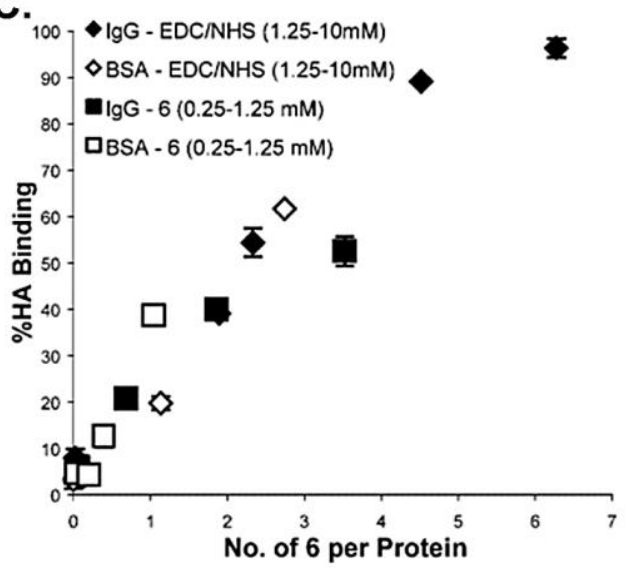

Figure 2.1 - DDS based on the assembly of multiple BPs. Images are republished with permission of Elsevier [35].

A. After activation of the carboxylic acid by carbodiimide chemistry, the di(bisphosphonate) (named here compound 6) can be covalently bound with the amine groups of proteins (represented by the grey sphere, being either bovine serum albumin or IgG).

B. Di(bisphosphonate)/protein conjugates resulted in strong dHAP affinity compared to pristine proteins (analyzed in vitro)

C. Degree of affinity was proportional to the amount of di(bisphosphonate) units bound to the protein.

Compared to BP compounds, (multi)-phosphonate-containing molecules are often designed as a targeting group for their conjugated drug load and not as standalone therapeutics, but some of them have been extensively employed for the transportation of radionuclides, which will be discussed in later sections of this review. 


\section{Tetracycline}

Tetracycline (TC, Table 2 compound $\mathrm{C}$ ) is an antibiotic produced by the actinobacterial genus Streptomyces and has been used as a therapeutic agent for decades. In addition to its antimicrobial properties, TC has also affinity to divalent cations such as $\mathrm{Ca}^{2+}$ present in dHAP. More specifically, TC accumulates on bone tissues where biological turnover is high, providing a tool to analyze bone propagation fronts as it also emits fluorescence under excitation at $390 \mathrm{~nm}[36]$. The $\beta$-diketone system at position 1 and 2 , the enol system at position 4 and 6 and the carboxamide group at position 5 are responsible for the chelating behavior of TC (Table 2, compound C) [37].

Research has focused on remodeling the tricarbonylmethane grouping in the A ring of TC [38], which is partly responsible for the molecule's affinity toward dHAP. The resulting 3-amino-2,6-dihydroxybenzamide ring structure exhibits a binding affinity increased of up to 50\% for dHAP compared to native TC [38]. Besides chelation between TC and dHAP, other interactions might contribute to their association. Van der Waals attractions and hydrogen bonding between the hydroxyl group of dHAP and TC molecules are likely to cause additional surface complexation [39].

As mentioned previously, TC staining is commonly used as a method to image and to quantify new bone formation, as it stains the surface of propagating bone formation front and has fluorescent properties [40,41]. For TC-functionalized DDS, this could result in reduced affinity to pathologic bone sites characterized by low bone turn-over. These factors could make TC a suboptimal candidate as BMS for DDS directed to bone-related diseases like osteomyelitis [42]. In addition, the chelation of TC is permanent, which can result in unwanted side effects such as staining of the teeth. 
Hence, TC is rarely used anymore for antibacterial purposes and prescribed with care to children still undergoing dental development [43].

\section{Bone-targeting peptides}

Oligopeptides of Aspartic acid (Asp) or Glutamic acid (Glu) have affinity toward dHAP [44], even though the exact mechanism behind is currently under debate [45]. It is known that a peptides affinity to dHAP increases when repeating units of Asp or Glu are present in the amino acid sequence, as it is naturally the case in osteopontin and osteocalcin bone-proteins [46] The utilization of acidic oligopeptides of Asp or Glu as bone seeking agents is an attractive option due to the fact that they have no apparent adverse effects and a shorter half-life in vivo compared to BPs [47].

Ishizakia et al. reported on the application of these acidic oligopeptides to transport various drugs: i.e. estradiol, quinolone antibiotics and tissue-nonspecific alkaline phosphatase (ALP) [44]. These compounds were conjugated by means of succinate esterification (estradiol and quinolones) or by changing the peptide sequence of ALP at the C-terminus. Interestingly, the authors stated that the measure of affinity between the oligopeptide and dHAP was not influenced by the choice of amino acid (Glu or Asp) or its optical isomer forms (D or L), but that dHAP affinity plateaued at six or more amino acids per oligomer [48]. Due to the non-hydrolysable nature of D-Glu and/or D-Asp rich-oligopeptides, its residence time at bone sites was reported to be longer compared to peptides in L configuration [48]. The structure of Asp can be further classified into $\alpha$ - and $\beta$-linkages between the monomers. Nakato et al. have analyzed the difference in chelating properties of polymeric Asp structures including $\alpha$-L-Asp, $\alpha$-D-Asp, $\beta$-L-Asp and $\alpha, \beta$ L-Asp [49]. They discovered that the $\operatorname{poly}(\alpha$-Asp) configuration had the 
highest chelation properties to $\mathrm{Ca}^{2+}$ ions due to the spatial location and configuration of the carboxyl groups on the polymer backbone. It was also confirmed that the chirality of the Asp had no effect on the chelation properties. It can be extrapolated that $\operatorname{poly}(\alpha-\mathrm{L} / \mathrm{D}-\mathrm{Asp})$ must have equal affinity toward dHAP, with desirable degradation properties from the poly $(\alpha-\mathrm{D}-\mathrm{Asp})$ configuration (see Table 2, compound $\mathrm{D})$. However, most of the literature does not report in the methodology the nature of the linkage present in the poly(Asp), making a comparison between the studies difficult to conduct.

Keeping in mind the vast possibilities in peptide combinations, other peptide sequences might have enhanced affinity to bone as well. In 2009, using phage display techniques, three peptides with the sequences VTKHLNQISQSY (VTK), STLPIPHEFSRE and APWHLSSQYSRT were identified as having strong and specific affinity toward dHAP and bone like material [50,51]. Additionally, follow-up studies have shown that biomaterials modified with VTK peptides favored osteogenic differentiation of human mesenchymal stem cells (hMSC) and biomineral deposition [52,53]. However, conflicting reports state that VTK peptides also have the ability to inhibit osteoblast mineralization [54], which could be a potential adverse effect. The adsorption mechanism of VTK peptides to dHAP has not been properly described. Surprisingly, the amino acids that are known to have affinity to dHAP (as mentioned above, i.e. Asp (D) and Glu (E)), are not present in this peptide sequence, and the net charge of the peptide sequence is in fact positive. Addison et al. emphasized the importance of the phosphorylation of the serine amino acids in the VTK peptide sequence on their binding energy required to interact to HAP, as phosphorous groups lower the molecular net charge which is beneficial for interactions with calcium [50]. 
This was further confirmed by computational modeling, which permitted to identify the amino acids responsible for binding to HAP crystals. This approach revealed as well that phosphorylated serine was almost always involved in dHAP binding, and that the hydroxyl side group of tyrosine also interacted with the crystalline surface. To the best of the authors' knowledge, no publications about VTK peptide conjugates to drug delivery systems or direct comparisons with other BMS have been published to date.

\section{Drug delivery systems using bone-seeking agents for targeting therapeutics}

\section{Prodrugs with bone affinity}

A prodrug is defined as a chemically modified drug that can be metabolized in the body into an active drug. Bone targeting prodrugs based on BMS have been developed to treat bone infection by grafting with antibiotics [55-57], or to treat osteoporosis by grafting with estrogen compounds like estradiol [38,58]. In 2008, Houghton et al. modified fluoroquinolones with BP groups, by linking the BP with the piperazine group of the fluoroquinolones [59]. The obtained chimeric bisphosphonated drugs are hydrophilic and highly water soluble due to the acidic nature of the BP moiety at physiological $\mathrm{pH}$ [60]. An in vivo investigation using a rat bone infection model revealed that bisphosphonated fluoroquinolones have a higher infection prevention rate compared to the systemically administered parent drug control [59]. One limitation of this conjugate system is that not all the prodrugs could dissociate to form the active antibiotic in clinically relevant quantities after its binding to HAP, due to slow hydrolysis of the antibiotic-DDS ester conjugation. While Houghton et al. utilized the piperazine group to link 
fluoroquinolones to BPs, Tanaka et al. used the carboxylic acid group of moxifloxacin, gatifloxacin and ciprofloxacin to generate their respective prodrug forms with BPs [56]. The same authors report on the production of bisphosphonated glycopetide antibiotic, (i.e. vancomycin and oritavancin) with a potential application for osteomyelitis [57]. In vitro experiments showed a high affinity toward bone for all prodrugs ( $>96.5 \%$ bone binding), but once more, only a small fraction of the prodrug was able to be converted into the active parent drug $(<3.5 \%$ in phosphate-buffered saline (PBS) after $24 \mathrm{hrs}$ [57], restricting the possibility to reach high local drug release. In rat serum, conversion to active antibiotic was higher (up to $26.4 \%$ ) due to enzymatic ester cleavage, which was presented as sufficient the for treatment purpose [57].

Bone seeking peptides linked to estradiol, an effective drug to stop or even to reverse osteoporotic phenomena $[61,62]$, have been the focus of extensive researches. Tokogawa et al. linked estradiol with L-Asp hexapeptide via succinate esterification, resulting in estradiol-17b-succinate-(L-aspartate) 6 $\left(\mathrm{E}_{2} \cdot 17 \mathrm{D}_{6}\right)$, for an intranasal administration application [62]. In addition, to enhance nasal uptake, conjugation of $\mathrm{E}_{2} \cdot 17 \mathrm{D}_{6}$ to absorption enhancers (e.g. $\beta$-cyclodextrin and hydroxypropyl cellulose) was performed. The results showed that 6 hours post-administration, the amount of estradiol increased in the bone due to the developed $\mathrm{E}_{2} \cdot 17 \mathrm{D}_{6}$ formulation, and that intranasal was a viable and attractive method of administration.

The fabrication of tetracycline-estradiol conjugates was reported by Orme et al. [58]. To link the bone seeker to the drug, a succinic anhydride linkage was made in presence of 4-dimethylaminopyridine (4-DMAP) catalyst during esterification reaction. The conjugate showed similar bone affinity compared 
to tetracycline, with over $99 \%$ of the compound bound to HAP in $60 \mathrm{~min}$. The authors assume the ester linkage between tetracycline and estradiol being degradable, essential to regenerate the parent drug; nonetheless, no further studies were found to validate parent drug recovery of tetracyclineestradiol conjugates.

\section{Modified polymer drug carriers}

Polymers, either natural or synthetic, are extensively studied materials as carriers to deliver drug to target tissues. With a broad range of biodegradable and biocompatible polymers, the physical, chemical and biological properties of polymer DDS can be highly tunable. In terms of drug release mechanism, polymeric DDS can be separated in four classes: diffusion controlled [63], solvent activated (swelling or osmotic regulated) [64], chemically controlled (degradation regulated) [63] or externally triggered systems (regulated by $\mathrm{pH}$ or temperature change) [65]; with some DDS being able to release their drug load in a synergetic manner $[63,66]$. Unfortunately, most polymers lack the intrinsic ability to target the desired tissue, but are subjectable to chemical functionalization with targeting moieties.

One of the main concerns with polymer/BMS-conjugates is the chemical alteration of the two components, which might alter the bone affinity of the BMS and/or change the desired properties of the polymer. For instance, for BPs to maintain bone affinity, the two phosphonate groups should not be sterically hindered during the binding of the molecule to the surface of polymer particle.

It is important to note that the second-generation BP Alendronate (ALN) is often used as a bone seeker covalently bound to polymer structures, due to 
its reactivity, sterically free primary amine group on the $\mathrm{R}_{2}$ side chain which is not involved in chelation processes $[67,68]$. The drug loaded polymer structures are commonly micro or nano-size particles or micellar structures functionalized with certain BPs.

\section{Solid micro- and nanospheres}

During the production of bioactive solid drug delivery particles, the drug is usually incorporated in the polymer matrix by dissolving the polymer and the pharmaceuticals in a common solvent or a co-solvent system before particles fabrication. Nanoprecipitation [69], emulsion [70], solvent displacement [71] or electrospraying techniques [72] are among the conventional methods, which ideally result in particles containing the drug homogeneously distributed throughout the bulk of the particles. The polymers can be functionalized by bioactive molecules (e.g. BP [68], peptides [73] or TC [74]) either before formulation of particles (e.g. chain ends modification [68]) or after the fabrication of particles by surface grafting $[75,76]$.

Choi et al. incorporated estrogen in nanospheres made of polylactic-coglycolic acid (PLGA) and monomethoxy polyethylene glycol (mPEG) copolymers (PLGA-mPEG) and PLGA with ALN grafted on the carboxylic end group. The rationale behind this dual-copolymer strategy was that the hydrophilic surface mPEG can increase the circulation time of the DDS due to the increased hydrodynamic diameter, and that the ALN would increase the site specificity of the particles to bone [68]. The fabrication of such particles required first the covalent grafting of ALN to PLGA using carbodiimide chemistry and secondly the synthesis of PLGA-mPEG. Subsequently, particles were fabricated using both polymers in a dialysis method without the addition of surfactants. The cumulative in vitro estrogen 
release in PBS over 60 hours was $80 \%$ of the initial loaded drug. The increase in $\mathrm{MPEG}$ chain length did not have a significant effect on the release profile of estrogen, but did result in a lower dHAP affinity. The authors hypothesized that long PEG chains could sterically hinder the ALN moiety to chelate to calcium in dHAP, but further optimization on MPEG chain length to have optimal systemic retention and conservation of strong dHAP binding is still needed [68].

A study by Chaudarhi et al. used zoledronate as a BMS for targeted delivery of docetaxel loaded PLGA nanoparticles (PLGA-NP) [77]. Solid PLGA docetaxel loaded particles were fabricated using nanoprecipitation after which the surface of the particles was functionalized with PEG chains and zoledronate moieties by NHS-dicyclohexylcarbodiimide (DCC) and N,N'Carbonyldiimidazole chemistry respectively. Using ${ }^{99 \mathrm{~m}} \mathrm{TC}$ labeling, they determined the blood/liver, bone/blood and tumor containing bone/healthy bone ratio of PLGA-NP accumulation. As expected, the PEGylated particles showed a decrease in liver uptake, while the particles functionalized with Zoledronate had a 7.5-times increase for bone/blood ratio 1 hour after intravenous administration. After 24 hours, a 504\% increase of Zoledronate functionalized particles was detected in bone tumor compared to bare PLGA particles, illustrating the increased retention of the DDS in cancerous bone.

Poly(Asp) can also be used to endow the surface of solid polymer particles with bone affine properties. Jiang et al. used mPEG-PLGA and maleimidemPEG-PLGA particles in a 9:1 ratio as a potential drug carrier. After particle formation, the maleimide end groups were tagged with Fluorescein isothiocyanate (FITC) labeled oligomer (FITC-Asp 7 Cys) by means of an 
alkylation reaction between the sulfhydryl terminal groups of the peptide and the ring opened maleimide, resulting in thioether bonds [45]. The affinity of these synthesized FITC-Asp 7 Cys conjugated nanoparticles (NP) to dHAP was tested by exposing a gel containing dHAP (Figure 2.2A) to the particles in dispersion. The resulting diminution of the absorbance intensity of the supernatant (from 100\% to 20\%) indicated a strong and specific interaction to dHAP (Figure 2.2A). In vitro exposure of the FITC-Asp 7 Cys conjugated NPs to matrix produced by human mesenchymal stem cells (hMSC) cultivated under osteogenic condition, indicated that the particles had a higher affinity to mineralized matrix, compared to matrix produced by hMSC in normal basic media (Figure 2.2B) [45]. The FITC-Asp 7 Cys conjugated NPs did not interact with C2C12 (myoblast cell-line) and Sw10 (immortalized Schwann cell cell-line) cell cultures (Figure 2.2C), suggesting again specificity of the DDS toward mineralized matrix. This was confirmed by in vivo experiments showing specificity to bone tissue (Figure 2.2D). 
A
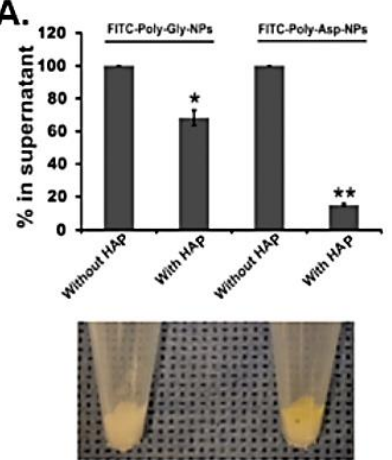

FITC-Poly-Gly-NPS FITC-Poly-Asp-NP

C.

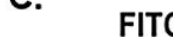

B.
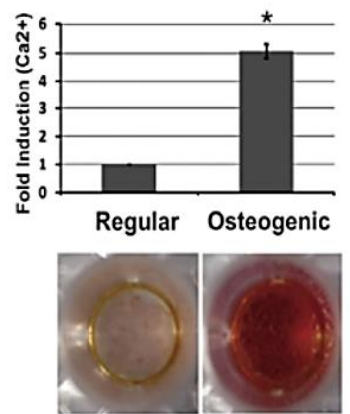

PI
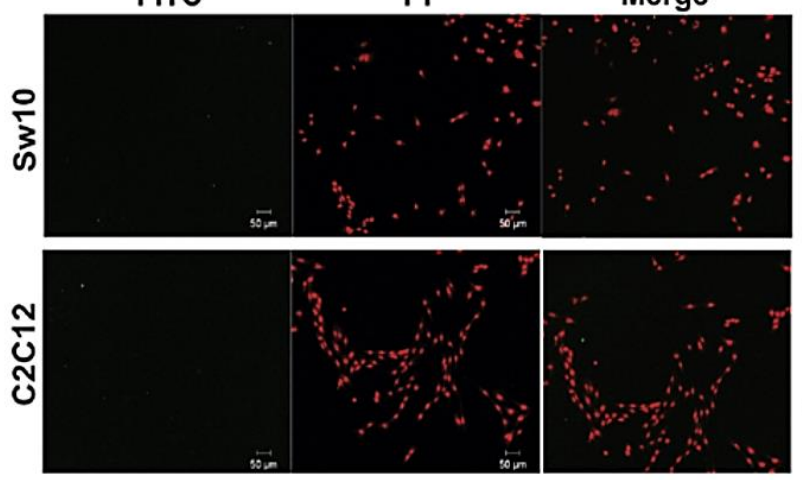
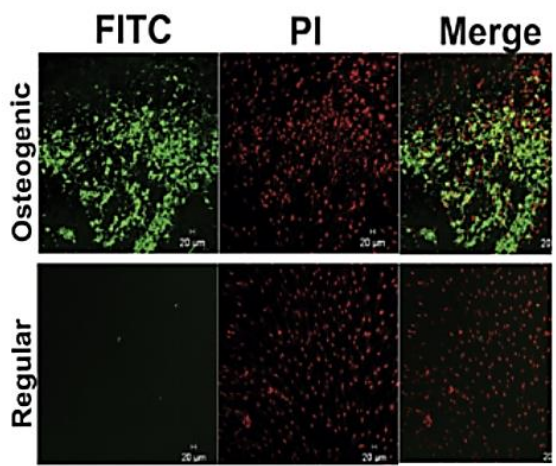

D.
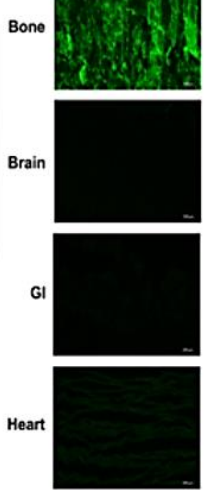

DIC
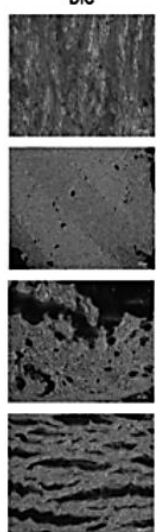

Merge
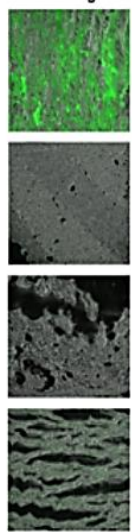

Figure 2.2 - DDS functionalized with poly(Asp) as therapeutic carrier for specific bone binding. All figures were modified and adapted with permission from [45].

A. The affinity of the FITC-Asp ${ }_{7}$ Cys conjugated NPs to HAP represented as the decrease in supernatant fluorescence of FITC-tagged particles, and illustration of the high HAP affinity of FITC-Asp ${ }_{7}$ Cys conjugated NPs (yellow color) and low affinity of FITC-Gly ${ }_{7}$ Cys conjugated NPs (whitish color).

B. FITC-Asp ${ }_{7}$ Cys conjugated NPs bind to mineralized matrix deposited by hMSCs cultured in osteogenic medium for 21 days (mineralized matrix was visualized by Alizarin red staining) (B).

C. On monolayer culture, no affinity was shown between FITC-Asp7Cys conjugated NPs and Sw10 or $\mathrm{C} 2 \mathrm{C} 12$ cell lines.

D. Histological samples of organ tissues from mice after systemic administration of FITC-Asp7Cys conjugated NPs, indicating that the NPs accumulated preferentially in the bone, while its presence was limited in other tissues. 


\section{Micro- and nanocapsules}

Micro- and nanocapsules can be defined as vesicular constructs with a typical core/shell structure [78]. The core can contain therapeutics in liquid, solid or dispersed form and the outer (polymer) hard shell provides protection from the biological environment and can provide targeting properties. There are several fabrication methods to produce capsules including nanoprecipitation [79], emulsion diffusion $[80,81]$ and double emulsification [81].

A

B.
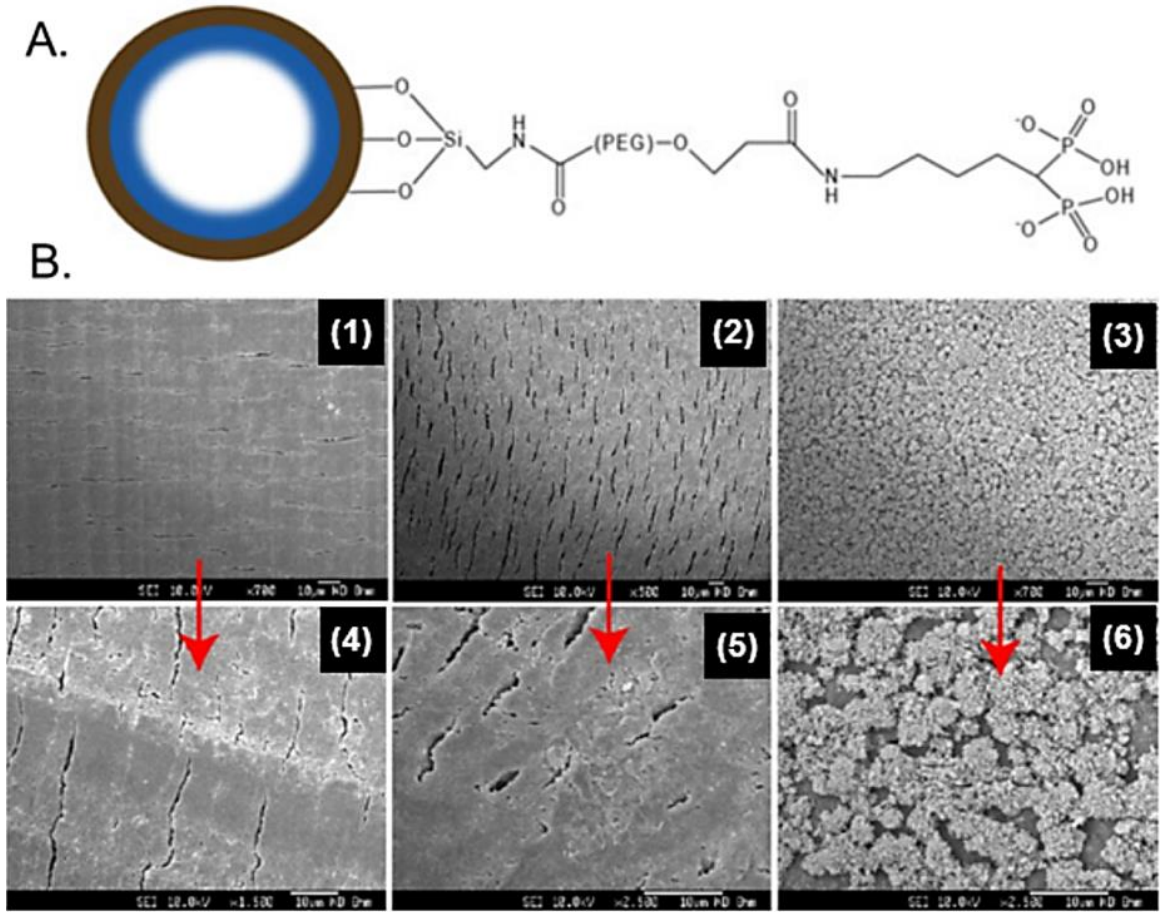

Figure 2.3 - Calcium phosphate (CaP) based DDS using BP for bone targeting, as described by Khung et al. [82].

A. CaP - sodium didicyl sulfate (SDS) nanocapsules were functionalized with silanePEG-NHS by silanisation processing, followed by esterification with an amine terminated BP.

B. Field emission scanning electron microscopy images of dentin discs with the addition of: (1) unmodified CaP nanocapsules, (2) PEG functionalized CaP nanocapsules, (3) BP functionalized CaP nanocapsules (with the images (4), (5) and (6) representing higher magnifications at 2500x). 
A limited amount of work has been published so far on BMS-nanocapsule conjugates. Khung et al. reported on the fabrication of calcium phosphate (CaP) nanocapsules with sodium dodecyl sulfate (SDS) coating (Figure 2.3) [82]. The silane group of silane-PEG-N-hydroxysuccinimide ester was conjugated with the outer SDS layer in a silanisation process. A coupling reaction occurred between an amino terminated $\mathrm{BP}$ and the $\mathrm{N}$ hydroxysuccinimide ester terminal group resulting in a CaP core/SDS shell nanocapsule with a silane-PEG-NHS linker that was BP functionalized (Figure 2.3A). An in vitro affinity study was performed (by incubating the nanocapsules with dentin discs), revealing an evident adsorption of only the BP functionalized nanocapsules (Figure 2.3B).

Very recently, Wang et al. have reported the fabrication and characterization of bone targeting Zeolitic imidazolate framework (ZIF) nanocapsules with a catechol modified gelatin as a wall material [83]. The authors could load the nanocapsules with hydrophobic Simvastatin with high encapsulation efficiency. The catechol groups allow for ALN to be implemented as a BMS after surface conjugation. In vitro experiments showed that the nanocapsules could be internalized by osteoblasts and exhibited affinity to dHAP. Compared to constructs without ALN, a 2.5-fold increase in nanocapsule accumulation in bone was shown after intravenous injections in rats.

\section{Dendrimers}

Dendrimers are spherical, branched molecular structures that can act as a carrier for drugs by entrapment of the pharmaceutical molecule in the void internal spaces or by association with the surface groups on the periphery of the dendrimer [84]. Dendrimers are often described in terms of generations (e.g. a $2^{\text {nd }}$ generation dendrimer consists of a core with branches whose end- 
groups also have further branched structures). Ouyang et al. presented the synthesis and in vitro bone binding characterization of various $2^{\text {nd }}$ and $3^{\text {rd }}$ generation poly(Asp) functionalized naproxen (anti-inflammatory drug) dendrimers [85]. It was hypothesized that the labile peptide bonds can be readily hydrolyzed, resulting in the release of parent drug at the site of bone infection. In a dHAP binding assay, the dendrimers showed an affinity greater than $60 \%$ within 2 hours.

Similarly, Cavero et al. reported on the synthesis of a $2^{\text {nd }}$ generation azabisphosphonate terminated dendrimers [86], with the prefix aza-indicating that the characteristic P-C-P backbone of the BP group was replaced with a P-N-P backbone. To create such multi-branched macromolecular structures, hexachlorophosphazene (HCP) was used as core (and as $1^{\text {st }}$ generation terminus) for further branching. The chlorine on HCP was substituted for the next generation of branched structures, nonetheless no in vitro or in vivo affinities to bone materials were reported in this study. For optimal BP chelation to calcium, regular BP end-groups are preferred over $a$ abisphosphonate derivatives, as the distance between the polar oxygen of the BPs might differ significantly and thus reduce the ability of the compound to effectively bind to calcium ions [21].

\section{Biomedical uses of bone mineral-seeking agent-modified drug delivery systems}

With the development of the previously mentioned drug delivery systems, many varieties of therapeutic agents that are administered systemically can now be accumulated at the region of interest. Several biomedical applications (either for diagnostic or for pathology treatments) have already benefited 
from such advanced bone-seeking drug delivery systems, and some of the FDA approved therapies, clinical trials under evaluation and the most promising developments still under pre-clinical evaluation will be later reported.

\section{Cancer: bone metastasis and osteosarcoma}

Bone is one of the most frequently affected tissues for cancers to metastasize [87]. Seventy percent of breast cancer metastasis occurs in bone tissue and prostate cancer mainly metastasizes in bone tissue $[88,89]$. Osteosarcoma (OS) (malignant cancer of the bone), is the most common primary tumor of bone tissue and affects mostly young people between the ages of 10 to 25 [90]. The most evident symptom characterizing OS is the unrestricted production of mineralized bone by tumor cells. The current gold standard for OS and bone metastasis treatment is a combination of surgical removal of the tumor and/or chemotherapy combining doxorubicin, methotrexate with leucovorin, cisplatin and ifosfimide. [91]. Despite the effectiveness of these compounds to stop the tumor cell replication, the drugs do not discriminate between tumor cells and healthy cells, resulting in severe systemic side effects. This makes the search for alternative treatments very attractive [92]. Radio-therapeutic treatment is another option to treat metastases in the body and can be done with external beam therapy or radionuclide drugs. Radioisotopes were first used for medical applications in 1940s and are considered one of the greatest medical advances of the $20^{\text {th }}$ century [93] and can be implemented in diagnostics, imaging purposes or radiotherapies. These wide range of applications makes transport of radiopharmaceuticals to bone sites clinically relevant. To prevent long term systemic damage to tissue surrounding the cancerous area, therapeutic bone 
targeting radiopharmaceuticals have a relatively short half-life ranging from several hours to multiple days [34]. Still, the systemic damage to healthy tissues is substantial and bone marrow toxicity (myelosuppression) is a general concern associated with radiotherapies [94].

Tomblyn et al. reports on seven radionuclides that are effective and safe for pain palliation in bone metastases, three of which are already approved for general clinical use [95]. The calcimimetic ${ }^{32} \mathrm{P}$ and ${ }^{89} \mathrm{Sr}$ radionuclides do not need to be conjugated to a bone seeker due to intrinsic affinity to bone. Calcimimetic ${ }^{223} \mathrm{Ra}$ is currently commercially available under the tradename Alpharadin ${ }^{\mathrm{TM}} .{ }^{153} \mathrm{Sm}$-EDTMP is an approved bone seeking conjugate for clinical applications is commercially available under several tradenames, including Lexidronam ${ }^{\mathrm{TM}}$. Some other radio-pharmaceuticals $\left(\right.$ e.g. ${ }^{223} \mathrm{Ra},{ }^{177} \mathrm{Lu}-$ EDTMP ${ }^{153} \mathrm{Sm}$-EDTMP) have also been used for palliative treatment of OS in order to decrease pain caused by bone metastases [96,97]. The mechanism behind the pain relief is currently not fully understood, however Lange et al. hypothesized that it can be attributed to the inhibition and killing of malignant cells [34]. For a comprehensive overview of recent developments in the field of radiopharmaceuticals and their delivery to bone, the authors recommend the review by Lange et al. [34]

When targeting tumors, including OS, one can take advantage of the ERP effect which allows larger molecules or constructs to cross the blood vessel membrane in cancer tissue. ${ }^{99 \mathrm{~m}} \mathrm{TC}$ carrying macromolecules consisting of a polymer backbone with polyphosphonate side chains were developed and tested in a canine OS model [98]. The difference in DDS uptake of osseous carcinomas and non-osseous carcinomas suggests that the passive EPR effect is not the only factor that plays a role in targeting OS. In fact, 
phosphonate groups present on the polymer can play an active role in targeting of OS due to their affinity with the calcium in dHAP that is highly present in these bone tumors [98].

Segal et al. reported on ALN and O-Chloracetyl-carbamoyl fumagillol (TNP470) conjugated to $\mathrm{N}$-(2-hydroxypropyl)methacrylamide (HPMA) designed for OS drug delivery [99] (Figure 2.4). TNP-470 is a potent anti-angiogenic agent aiming to reduce vascularization of OS induced tumors. ALN and TNP-470 were attached to the polymer backbone by cleavable peptide linkers that released the ALN and TNP-470 in vivo. It was hypothesized that the anti-angiogenesis properties of the TNP-470 and the tumor regressive properties of ALN could both contribute to treat OS. As a xenogeneic model of human OS, balb/c mice bearing K7M2 murine OS in the tibia were chosen. Bio-distribution study conducted after subcutaneous injection of the targeting conjugate in the mentioned pathogenic mice model indicated that this DDS can effectively target OS sites (Figure 2.4A and 2.4C). In comparison, kidneys and liver tissue expressed high uptake of non-targeting control conjugates due to blood filtering and the presence of highly permeable sinusoidal blood vessels in the liver (Figure 2.4B and 2.4C). 

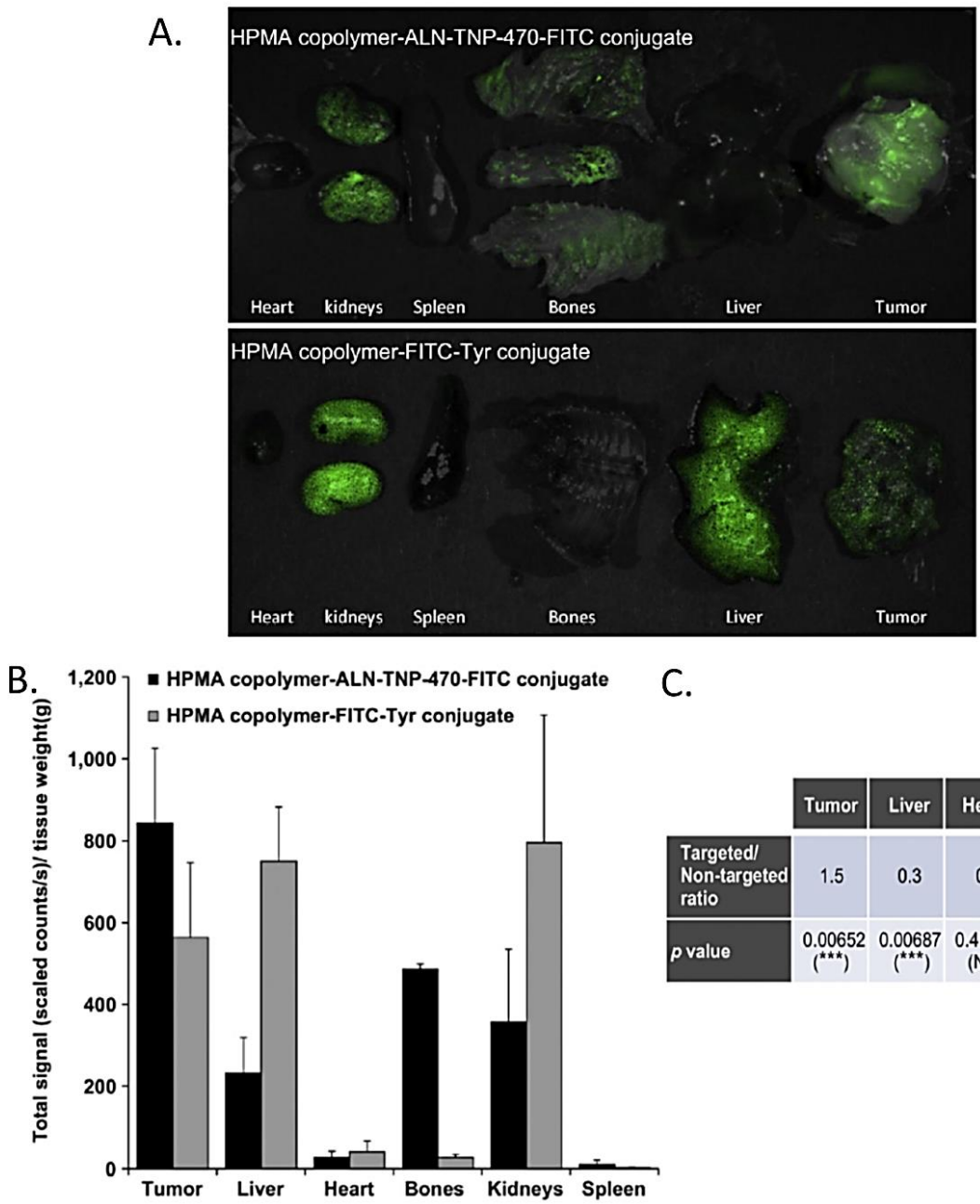

C.

\begin{tabular}{|c|c|c|c|c|c|c|}
\hline & Tumor & Liver & Heart & Bones & Kidneys & Spleen \\
\hline $\begin{array}{l}\text { Targeted/ } \\
\text { Non-targeted } \\
\text { ratio }\end{array}$ & 1.5 & 0.3 & 0.6 & 19 & 0.4 & 3.4 \\
\hline$p$ value & $\begin{array}{c}0.00652 \\
\left({ }^{* k \hbar}\right)\end{array}$ & $\begin{array}{c}0.00687 \\
\left({ }^{\star \star \star}\right)\end{array}$ & $\begin{array}{c}0.41891 \\
\text { (NS) }\end{array}$ & $\frac{0.00017}{\left(^{\star \star k}\right)}$ & $\begin{array}{c}0.04609 \\
\left({ }^{*}\right)\end{array}$ & $\begin{array}{c}0.49431 \\
\text { (NS) }\end{array}$ \\
\hline
\end{tabular}

Figure 2.4 - Delivery systems with bone affinity for OS treatment. All figures were modified and adapted with permission from [99]

A. Bio-distribution of ALN-copolymer-TNP 470 revealed preferential accumulation in bones and tumor in a mice model. The targeting ability of the FITC-ALNconjugate was evident by the increased fluorescence in the bone sample compared to the non-targeting copolymers.

B \& C. The non-targeting conjugate was cleared by the liver in significantly greater amount than the targeted conjugate, whereas the FITC-ALN-conjugate bound preferentially to bone tissues. 
The work of Qiao et al. describes the development of mesoporous silica covered Gadolinium(III) up-conversion nanoparticles (UCZP) which combine both diagnostic and therapeutic applications for early (breast cancer) metastases into bone [100]. The surface of these UCZP are functionalized with the BP Zolendronate and poly(acrylic acid), a $\mathrm{pH}$ responsive polymer. The diagnostic properties of UCZP were tested in an in vivo mouse model where malignant cells were deposited in the heart of the mice, mimicking bone metastasis of breast cancer. Two weeks after intracardial injection, the sites of metastasis could be identified by noninvasive in vivo bioluminescence imaging and magnetic resonance imaging of the UCZP. With X-ray imaging bone metastasis could identified significantly later, after three weeks. The therapeutic capabilities of the UCZP in the form of a DDS for Plumbagin were showed by high encapsulation efficiencies of the drug and by increased Plumbagin release in acidic environments, like the site of bone metastases. Plumbagin acts as a suppressor of osteocyte-related osteoclastogenesis by inhibiting expression of nuclear $x \mathrm{~B}$ ligand (RANKL) and Sclerostin. A reduced recruiting of Osteoclasts was observed, suggesting the effectiveness of the reported UCZP DDS to diminish the processes that enable breast cancer related bone metastases [100].

\section{Osteomyelitis}

Osteomyelitis (OM), infection of bone and bone marrow, can be categorized in chronic- and acute OM. Despite the nomenclature, the defining characteristic of $\mathrm{OM}$ classification does not include the duration of the infection, but rather the histopathologic features [42]. Chronic OM includes the presence of necrotic bone and is generally a consequence of an open fracture, bacteremia or contiguous soft tissue infection that has not been 
treated or treated unsuccessfully [42]. The incidence of chronic OM is increasing due to higher prevalence of predisposing factors like diabetes mellitus and peripheral vascular diseases [42]. The gold standard of treatment of chronic OM is a surgical debridement of necrotic bone and surrounding infected tissue, followed by several weeks of systemic antibiotic treatment [42,101-103]. It has been stated repeatedly that there are no convincing clinical studies showing that long-term duration of systemic antibiotic treatment is more effective than shorter therapies, however 4 to 6 weeks of therapy is used based on empirical findings [102,104].

As was introduced earlier, a local DDS that releases antibiotics over time might be preferable over systemic therapies. For such purposes, PMMA bone cements have been used in clinics for decades to keep prostheses in place and to prevent or treat infections on a local level [7,105]. Unfortunately, PMMA is non-biodegradable, can only be loaded with a limited number of heat stable antibiotics due to its exothermic polymerization properties and has an incomplete release of its loaded drug content $[7,105]$.

As previously mentioned, prodrugs based on bisphosphonate modified antibiotics have been developed with the goal to reduce the systemic dosage and increase the presence of the parent antibiotic at bony sites [28,55,59]. Moxifloxacin, gatifloxacin, and ciprofloxacin have a free amino position in which tethering with a BP group is a possible approach. Indeed, in vivo studies showed that a single dose of BP-gatifloxacin conjugates could prevent $\mathrm{OM}$ in a prophylactic OM rat model due to a longer presence of the antibiotic at the bone site [59].

Acidic oligopeptides have also been linked to quinolone antibiotics to treat osteomyelitis [106]. For instance, levofloxacin (LVFX) was linked to 
oligopeptide (L-Asp ${ }_{6}$ ) resulting in LVFX-D ${ }_{6}$ conjugates. A second antibiotic, Norfloxacin (NFLX), was conjugated in similar fashion (NFLX-D $)_{6}$, which showed increased in vitro affinity toward dHAP compared to the free quinolones, however LVFX-D 6 did not show similar properties [106]. The authors stated that the 3-carboxylate and 4-carbonyl groups in quinolones are also responsible for the chelating properties to divalent metals in bone, such as calcium. This explains their main findings as during the conjugation of LVFX-D $\mathrm{D}_{6}$ these groups are compromised while they remain unaltered during the formation of NFLX-D. In a mouse with OM, colony forming unit (CFU) count decreased of approximately 3-fold compared to untreated control mice and the LVFX-D 6 possessed antimicrobial activity up for 6 days. In contrast, the unconjugated LVFX group could not slow down bacteria proliferation and an increase of CFU after only 4 days was observed. Although LVFX-D 6 induced a significant CFU decrease, it might hardly be sufficient as a monotherapy agent and future applications as co-therapy would most likely to be envisioned.

To summarize, prodrugs with bone-affinity are still the main focus of newly marketed DDS to treat OM. Advances in the field of polymer based DDS with bone-targeting moieties, other than prodrugs, could bring clear benefits, but to the best of our knowledge, such advanced therapeutics have neither been FDA-approved, nor reported as being in advanced stage in the literature.

\section{Osteoporosis}

Osteoporosis (OP) is a bone pathology with a prevalence of 10 million people in the US alone. Over 2 million of bone fractures per year are a direct cause of the low bone density of patients affected by OP, and associated 
medical costs are expected to be as high as $\$ 25$ billion US dollar annually in 2025 [107,108]. General strategies to treat OP are either to decrease osteoclast activity, to stimulate the formation of new bone, or a combination of the both. The BP bone seeker class (e.g. ALN) is often used as a treatment to inhibit the dissolution of dHAP crystals by osteoclasts during the disturbed bone homeostasis in osteoporosis patients [21,30]. Currently, the only FDA approved anabolic drug for new bone formation is parathyroid hormone $(\mathrm{PTH})$, commercially available under the tradename Teriparatide ${ }^{\mathrm{TM}}$ [109]. Nonetheless, BP therapy is still the norm when treating OP and only a limited amount of advanced DDS combined to BMS have been developed.

As a treatment for Pagets disease and a second line therapy for postmenopausal OP, salmon calcitonin (sCT) is used to inhibit osteoclast bone resorbtion. With a 40 fold increase in osteoclast inhibition potency and a longer biological half-life compared to human calcitonin, sCT is a shortchain peptide with promising properties [110,111]. Due to low oral bioavailability, intravenous injections have been the prevered method of administration [111]. To increase the biological half-life of sCT, PEGylation of the protein can be performed [112], and to specifically improve the bone targeting property, Bhandari et al.conjugated such sCTwith a BP moiety, which resulted in an prolonged presence (on an in vitro HAP binding assay) [113].

As novel OP treatment, Wang et al. functionalized PLGA NP nanoparticles with TC [74]. TC-PLGA NP had a significantly higher affinity toward dHAP compared to PLGA NP, which revealed that grafting TC on macromolecules does not inhibit TC chelating activity. As a pro-osteogenic drug model, simvastatin (SIM) was loaded in the TC-PLGA. Following incubation with 
osteoblast precursor cell line (MC3T3-E1) those particles underwent lower cellular compared to free-TC controls NP, which had a direct influence on the degree of osteogenic differentiation of the cell line. The authors argued that the increasing hydrophilicity of the TC grafted NP resulted in a decrease of cellular internalization of the NP. Around $80 \%$ of the loaded SIM was released in 72 hours, leading to high concentrations of drug in the bone. SIM loaded TC-PLGA NP efficiently deliver SIM to the bone where it partially restores the bone mineral density (BMD) of ovariectomized rats. Of interest, they observed that femur and femoral head had a different response to the delivered SIM, which was explained by the fact that the cancellous bone in the femoral head is more susceptible to the drug's influence on osteoblast differentiation due to its greater surface area, compared to the denser cortical bone more prevalent in the femur [74].

\section{Conclusions}

Bone mineral seekers (BMS) are powerful (macro)-molecular tools to improve the delivery of therapeutic compounds to hydroxyapatite in bones. Among the described BMS, bisphosphonates, tetracycline and the oligopeptides poly(Asp) and poly(Glu) are the most frequently reported. The latter providing a promising alternative to un-reversible BP chelation. DrugBMS conjugates show significant increased accumulation and retention at bony sites but need to effectively release the parent drug in an appropriate time frame. For this reason, a significant amount of research has been dedicated to drug delivery systems implementing BMS, which have the benefits to protect the encapsulated therapeutic compounds and to guide the conjugate to bone for an efficient local drug release. Bone pathologies like osteosarcoma, osteomyelitis and osteoporosis can benefit from the 
mentioned functionalized delivery vehicles to optimize therapies and to limit plaguing side effects. Although many studies have shown promising in vivo results, few BMS modified DDS have successfully made translation to the clinics yet. Nevertheless, with the great amount of promising pre-clinical studies, with the main ones challenged in this review, we can envision an increase in BMS modified DDS to enter the clinics in the following years to alleviate current limitations of bone therapies and to relieve affected patients.

\section{Acknowledgements}

This work was supported by the AOTrauma Clinical Priority Program Bone Infection.

\section{References}

1 Gu, L. et al. Polyamidomine dendrimers: an excellent drug carrier for improving the solubility and bioavailability of puerarin. Pharm Dev Technol 18, 1051-1057, doi:10.3109/10837450.2011.653822 (2013).

2 Ankit, B., Gupta, S. \& Noble, B. Oral Sustained Release Dosage Form: An Opporturnity to Prolong the Release of Drug. IJARPB (2013).

3 Twibanire, J. D. K. \& Grindley, T. B. Polyester Dendrimers: Smart Carriers for Drug Delivery. Polymers-Basel 6, 179-213, doi:10.3390/polym6010179 (2014).

4 Fahmy, T. M., Fong, P. M., Goyal, A. \& Saltzman, W. M. Targeted for drug delivery. Materials Today 8, 18-26, doi:https://doi.org/10.1016/S13697021(05)71033-6 (2005).

5 Hughes, S. P. \& Anderson, F. M. Penetration of antibiotics into bone. J Antimicrob Chemother 15, 517-519 (1985).

6 Nandi, S. K. et al. Understanding osteomyelitis and its treatment through local drug delivery system. Biotechnology Advances, doi:http://dx.doi.org/10.1016/j.biotechadv.2016.09.005.

7 ter Boo, G.-J. A., Grijpma, D. W., Moriarty, T. F., Richards, R. G. \& Eglin, D. Antimicrobial delivery systems for local infection prophylaxis in orthopedic- and trauma surgery. Biomaterials $\quad \mathbf{5 2}$ 113-125, doi:https://doi.org/10.1016/j.biomaterials.2015.02.020 (2015).

8 Kuehn, K.-D., Ege, W. \& Gopp, U. Acrylic bone cements: Composition and properties. Vol. 36 (2005).

9 Landersdorfer, C. B., Bulitta, J. B. \& Sörgel, F. in Bone and Joint Infections 21-37 (John Wiley \& Sons, Ltd, 2015).

10 Fleisch, H. Bisphosphonates in bone disease: From laboatory to the patient. 2nd edn, (The Parthenon publishing group Ltd., 1995). 
11 Affinity of fibronectin to collagens of different genetic types and to fibrinogen. The Journal of Experimental Medicine 147, 1584-1595 (1978).

12 Di Lullo, G. A., Sweeney, S. M., Körkkö, J., Ala-Kokko, L. \& San Antonio, J. D. Mapping the Ligand-binding Sites and Disease-associated Mutations on the Most Abundant Protein in the Human, Type I Collagen. Journal of Biological Chemistry 277, 4223-4231, doi:10.1074/jbc.M110709200 (2002).

13 Metavarayuth, K., Sitasuwan, P., Luckanagul, J. A., Feng, S. \& Wang, Q. Virus Nanoparticles Mediated Osteogenic Differentiation of Bone Derived Mesenchymal Stem Cells. Advanced Science 2, 1500026-n/a, doi:10.1002/advs.201500026 (2015).

14 Torreggiani, E. et al. Preosteocytes/Osteocytes Have the Potential to Dedifferentiate Becoming a Source of Osteoblasts. Plos One 8, e75204, doi:10.1371/journal.pone.0075204 (2013).

15 Carbone, E. J. et al. Osteotropic nanoscale drug delivery systems based on small molecule bone-targeting moieties. Nanomedicine, doi:10.1016/j.nano.2016.08.015 (2016).

16 Wang, D., Miller, S. C., Kopečková, P. \& Kopeček, J. Bone-targeting macromolecular therapeutics. Advanced Drug Delivery Reviews 57, 1049-1076, doi:http://dx.doi.org/10.1016/j.addr.2004.12.011 (2005).

17 Ross, R. D. \& Roeder, R. K. Binding affinity of surface functionalized gold nanoparticles to hydroxyapatite. Journal of Biomedical Materials Research Part A 99A, 58-66, doi:10.1002/jbm.a.33165 (2011).

18 Ross, R. D., Cole, L. E. \& Roeder, R. K. Relative binding affinity of carboxylate-, phosphonate-, and bisphosphonate-functionalized gold nanoparticles targeted to damaged bone tissue. J Nanopart Res 14, 1175, doi:10.1007/s11051-012-1175-z (2012).

19 Fleisch, H., Russell, R. G., Bisaz, S., Casey, P. A. \& Muhlbauer, R. C. The influence of pyrophosphate analogues (diphosphonates) on the precipitation and dissolution. Calcif Tissue Res, Suppl:10-10a (1968).

20 Brown, J. P. et al. Bisphosphonates for treatment of osteoporosis: Expected benefits, potential harms, and drug holidays. Canadian Family Physician 60, 324-333 (2014).

21 Yewle, J. N. BIFUNCTIONAL BISPHOSPHONATES FOR DELIVERING BIOMOLECULES TO BONE PhD thesis, (2012).

22 Zhang, S., Gangal, G. \& Uludag, H. 'Magic bullets' for bone diseases: progress in rational design of bone-seeking medicinal agents. Chemical Society Reviews 36, $507-$ 531, doi:10.1039/B512310K (2007).

23 Lawson, M. A. et al. Differences between bisphosphonates in binding affinities for hydroxyapatite. J Biomed Mater Res B Appl Biomater 92, 149-155, doi:10.1002/jbm.b.31500 (2010).

24 Nancollas, G. H. et al. Novel insights into actions of bisphosphonates on bone: differences in interactions with hydroxyapatite. Bone 38, 617-627, doi:10.1016/j.bone.2005.05.003 (2006).

25 Chen, J. S. \& Sambrook, P. N. Antiresorptive therapies for osteoporosis: a clinical overview. Nat Rev Endocrinol 8, 81-91 (2012).

26 Drake, M. T., Clarke, B. L. \& Khosla, S. Bisphosphonates: Mechanism of Action and Role in Clinical Practice. Mayo Clinic proceedings. Mayo Clinic 83, 1032-1045 (2008). 
Puljula, E. et al. Structural requirements for bisphosphonate binding on hydroxyapatite: NMR study of bisphosphonate partial esters. ACS Med Chem Lett 6, 397-401, doi:10.1021/ml5004603 (2015).

Monteil, M., Migianu-Griffoni, E., Sainte-Catherine, O., Di Benedetto, M. \& Lecouvey, M. Bisphosphonate prodrugs: synthesis and biological evaluation in HuH7 hepatocarcinoma cells. Eur J Med Chem 77, 56-64, doi:10.1016/j.ejmech.2014.02.054 (2014).

Morton, S. W. et al. Osteotropic therapy via targeted layer-by-layer nanoparticles. Adv Healthc Mater 3, 867-875, doi:10.1002/adhm.201300465 (2014).

30 Brown, J. P. et al. Bisphosphonates for treatment of osteoporosis: expected benefits, potential harms, and drug holidays. Can Fam Physician 60, 324-333 (2014). Kuhl, S., Walter, C., Acham, S., Pfeffer, R. \& Lambrecht, J. T. Bisphosphonaterelated osteonecrosis of the jaws--a review. Oral Oncol 48, 938-947, doi:10.1016/j.oraloncology.2012.03.028 (2012).

Alendronate - FDA prescribing information, side-effects and usages, $<$ https://www.drugs.com/pro/alendronate.html> (2017).

Yin, Q. et al. Pamidronate functionalized nanoconjugates for targeted therapy of focal skeletal malignant osteolysis. Proceedings of the National Academy of Sciences of the United States of America 113, E4601-4609, doi:10.1073/pnas.1603316113 (2016).

4 Lange, R. et al. Pharmaceutical and clinical development of phosphonate-based radiopharmaceuticals for the targeted treatment of bone metastases. Bone 91, 159179, doi:10.1016/j.bone.2016.08.002 (2016).

5 Bansal, G., Gittens, S. A. \& Uludağ, H. A di(bisphosphonic acid) for protein coupling and targeting to bone. Journal of Pharmaceutical Sciences 93, 2788-2799, doi:10.1002/jps.20186 (2004).

6 Tam, C. S. \& Anderson, W. Tetracycline labeling of bone in vivo. Calcif Tissue Int 30, 121-125 (1980).

7 Chopra, I. \& Roberts, M. Tetracycline antibiotics: mode of action, applications, molecular biology, and epidemiology of bacterial resistance. Microbiol Mol Biol Rev 65, 232-260 ; second page, table of contents, doi:10.1128/MMBR.65.2.232260.2001 (2001).

8 Neale, J. R. et al. Bone selective effect of an estradiol conjugate with a novel tetracycline-derived bone-targeting agent. Bioorg Med Chem Lett 19, 680-683, doi:10.1016/j.bmcl.2008.12.051 (2009).

Wang, J. T., Hu, J. \& Zhang, S. W. Studies on the sorption of tetracycline onto clays and marine sediment from seawater. J Colloid Interf Sci 349, 578-582, doi:10.1016/j.jcis.2010.04.081 (2010).

Cochran, M., Neville, A. \& Marshall, E. A. Comparison of bone formation rates measured by radiocalcium kinetics and double-tetracycline labeling in maintenance dialysis patients. Calcified Tissue International 54, 392-398, doi:10.1007/bf00305526 (1994).

Frost, H. M., Villanueva, A. R., Roth, H. \& Stanisavljevic, S. Tetracycline Bone Labeling. The Journal of New Drugs 1, 206-216, doi:10.1177/009127006100100503 (1961).

2 Hatzenbuehler, J. \& Pulling, T. J. Diagnosis and management of osteomyelitis. American family physician 84, 1027-1033 (2011).

Cutbirth, S. T. A Restorative Challenge: Tetracycline-Stained Teeth. Dent Today 34, 126, 128-130 (2015). 
44 Ishizaki, J., Waki, Y., Takahashi-Nishioka, T., Yokogawa, K. \& Miyamoto, K. Selective drug delivery to bone using acidic oligopeptides. Journal of Bone and Mineral Metabolism 27, 1-8, doi:10.1007/s00774-008-0004-z (2009). Jiang, T. et al. Poly aspartic acid peptide-linked PLGA based nanoscale particles: Potential for bone-targeting drug delivery applications. International Journal of Pharmaceutics 475, 547-557, doi:https://doi.org/10.1016/j.ijpharm.2014.08.067 (2014).

46 Tavafoghi, M. \& Cerruti, M. The role of amino acids in hydroxyapatite mineralization. J R Soc Interface 13, doi:10.1098/rsif.2016.0462 (2016).

47 Kasugai, S., Fujisawa, R., Waki, Y., Miyamoto, K. \& Ohya, K. Selective drug delivery system to bone: small peptide (Asp)6 conjugation. J Bone Miner Res 15, 936943, doi:10.1359/jbmr.2000.15.5.936 (2000).

48 Sekido, T. et al. Novel drug delivery system to bone using acidic oligopeptide: pharmacokinetic characteristics and pharmacological potential. J Drug Target 9, 111-121 (2001).

49 Nakato, T., Yoshitake, M., Matsubara, K., Tomida, M. \& Kakuchi, T. Relationships between Structure and Properties of Poly(aspartic acid)s. Macromolecules 31, 2107 2113, doi:10.1021/ma971629y (1998).

50 Addison, W. N. et al. Phosphorylation-dependent mineral-type specificity for apatite-binding peptide sequences. Biomaterials 31, 9422-9430, doi:10.1016/j.biomaterials.2010.08.064 (2010).

51 Segvich, S. J., Smith, H. C. \& Kohn, D. H. The adsorption of preferential binding peptides to apatite-based materials. Biomaterials 30, 1287-1298, doi:http://dx.doi.org/10.1016/i.biomaterials.2008.11.008 (2009).

52 Ramaraju, H. \& Kohn, D. H. Phage display derived peptides increase human MSC mediated bone formation in vivo. Frontiers in Bioengineering and Biotechnology, doi:10.3389/conf.FBIOE.2016.01.00452.

53 Dinjaski, N. et al. Osteoinductive recombinant silk fusion proteins for bone regeneration. Acta Biomaterialia, doi:http://dx.doi.org/10.1016/i.actbio.2016.12.002.

54 Ramaswamy, J., Nam, H. K., Ramaraju, H., Hatch, N. E. \& Kohn, D. H. Inhibition of osteoblast mineralization by phosphorylated phage-derived apatite-specific peptide. Biomaterials 73, 120-130, doi:10.1016/j.biomaterials.2015.09.021 (2015).

55 Reddy, R. et al. Bisphosphonated Benzoxazinorifamycin Prodrugs for the Prevention and Treatment of Osteomyelitis. ChemMedChem 3, 1863-1868, doi:10.1002/cmdc.200800255 (2008).

56 Tanaka, K. S. E. et al. Bisphosphonated fluoroquinolone esters as osteotropic prodrugs for the prevention of osteomyelitis. Bioorganic \& Medicinal Chemistry 16, 9217-9229, doi:http://dx.doi.org/10.1016/j.bmc.2008.09.010 (2008).

57 Tanaka, K. S. E. et al. Synthesis and in vitro evaluation of bisphosphonated glycopeptide prodrugs for the treatment of osteomyelitis. Bioorganic \& Medicinal Chemistry Letters 20, 1355-1359, doi:http://dx.doi.org/10.1016/j.bmcl.2010.01.006 (2010).

58 Orme, M. W. \& Labroo, V. M. Synthesis of $\beta$-estradiol-3-benzoate-17-(succinyl12A-tetracycline): A potential bone-seeking estrogen. Bioorganic \& Medicinal Chemistry Letters 4, 1375-1380, doi:http://dx.doi.org/10.1016/S0960$\underline{894 X(01) 80365-6}$ (1994). 
Houghton, T. J. et al. Linking Bisphosphonates to the Free Amino Groups in Fluoroquinolones: Preparation of Osteotropic Prodrugs for the Prevention of Osteomyelitis. Journal of Medicinal Chemistry 51, 6955-6969, doi:10.1021/jm801007z (2008).

60 Hirabayashi, H. \& Fujisaki, J. Bone-specific drug delivery systems: approaches via chemical modification of bone-seeking agents. Clin Pharmacokinet 42, 1319-1330, doi:10.2165/00003088-200342150-00002 (2003).

61 Komm, B. et al. Estrogen binding, receptor mRNA, and biologic response in osteoblast-like osteosarcoma cells. Science 241, 81-84, doi:10.1126/science.3164526 (1988).

62 Yokogawa, K. et al. Pharmacokinetic advantage of an intranasal preparation of a novel anti-osteoporosis drug, L-Asp-hexapeptide-conjugated estradiol. Biol Pharm Bull 29, 1229-1233 (2006).

63 Posadowska, U., Brzychczy-Wloch, M. \& Pamula, E. Gentamicin loaded PLGA nanoparticles as local drug delivery system for the osteomyelitis treatment. Acta of Bioengineering and Biomechanics 17, 41-48, doi:10.5277/Abb-00188-2014-02 (2015).

64 Verma, R. K., Mishra, B. \& Garg, S. Osmotically controlled oral drug delivery. Drug Dev Ind Pharm 26, 695-708, doi:10.1081/ddc-100101287 (2000).

65 Liechty, W. B., Kryscio, D. R., Slaughter, B. V. \& Peppas, N. A. Polymers for drug delivery systems. Annu Rev Chem Biomol Eng 1, 149-173, doi:10.1146/annurevchembioeng-073009-100847 (2010).

66 Asghar, W. et al. PLGA Micro- and Nanoparticles Loaded Into Gelatin Scaffold for Controlled Drug Release. Ieee Transactions on Nanotechnology 11, 546-553, doi:10.1109/Tnano.2012.2183004 (2012).

67 de Miguel, L., Noiray, M., Surpateanu, G., Iorga, B. I. \& Ponchel, G. Poly( $\gamma$-benzyll-glutamate)-PEG-alendronate multivalent nanoparticles for bone targeting. International Journal of Pharmaceutics 460, 73-82, doi:http://dx.doi.org/10.1016/j.ijpharm.2013.10.048 (2014).

68 Choi, S. W. \& Kim, J. H. Design of surface-modified poly(D,L-lactide-coglycolide) nanoparticles for targeted drug delivery to bone. J Control Release 122, 24 30, doi:10.1016/j.jconrel.2007.06.003 (2007).

69 Govender, T., Stolnik, S., Garnett, M. C., Illum, L. \& Davis, S. S. PLGA nanoparticles prepared by nanoprecipitation: drug loading and release studies of a water soluble drug. J Control Release 57, 171-185 (1999).

70 Posadowska, U., Brzychczy-Wloch, M. \& Pamula, E. Gentamicin loaded PLGA nanoparticles as local drug delivery system for the osteomyelitis treatment. Acta Bioeng Biomech 17, 41-48 (2015).

71 Jia, W. et al. Preparation of biodegradable polycaprolactone/poly (ethylene glycol)/polycaprolactone (PCEC) nanoparticles. Drug Deliv 15, 409-416, doi:10.1080/10717540802321727 (2008).

72 Bock, N., Woodruff, M. A., Hutmacher, D. W. \& Dargaville, T. R. Electrospraying, a Reproducible Method for Production of Polymeric Microspheres for Biomedical Applications. Polymers-Basel 3, 131-149, doi:10.3390/polym3010131 (2011).

73 Tudorachi, N., Lipsa, R. D., Vasile, C. \& Mustata, F. Poly(Lactic Acid)-co-Aspartic Acid Copolymers: Possible Utilization in Drug Delivery Systems. Vol. 21 (2014).

74 Wang, H. et al. Tetracycline-grafted PLGA nanoparticles as bone-targeting drug delivery system. Int J Nanomed 10, 5671-5685, doi:10.2147/Ijn.S88798 (2015). 
75 Li, N., Song, J., Zhu, G., Shi, X. \& Wang, Y. Alendronate conjugated nanoparticles for calcification targeting. Colloids and Surfaces B: Biointerfaces 142, 344-350, doi:https://doi.org/10.1016/i.colsurfb.2016.03.015 (2016).

76 Thamake, S. I., Raut, S. L., Gryczynski, Z., Ranjan, A. P. \& Vishwanatha, J. K. Alendronate coated poly-lactic-co-glycolic acid (PLGA) nanoparticles for active targeting of metastatic breast cancer. Biomaterials 33, 7164-7173, doi:http://dx.doi.org/10.1016/i.biomaterials.2012.06.026 (2012).

77 Chaudhari, K. R. et al. Bone metastasis targeting: A novel approach to reach bone using Zoledronate anchored PLGA nanoparticle as carrier system loaded with Docetaxel. Journal of Controlled Release 158, 470-478, doi:10.1016/j.jconrel.2011.11.020 (2012).

78 Mora-Huertas, C. E., Fessi, H. \& Elaissari, A. Polymer-based nanocapsules for drug delivery. Int J Pharm 385, 113-142, doi:10.1016/j.ijpharm.2009.10.018 (2010).

79 Fessi, H., Puisieux, F., Devissaguet, J. P., Ammoury, N. \& Benita, S. Nanocapsule formation by interfacial polymer deposition following solvent displacement. International Journal of Pharmaceutics 55, R1-R4, doi:https://doi.org/10.1016/03785173(89)90281-0 (1989).

80 Quintanar-Guerrero, D., Allémann, E., Doelker, E. \& Fessi, H. Preparation and Characterization of Nanocapsules from Preformed Polymers by a New Process Based on Emulsification-Diffusion Technique. Pharmacentical Research 15, 10561062, doi:10.1023/A:1011934328471 (1998).

81 Perez, C. et al. Poly(lactic acid)-poly(ethylene glycol) nanoparticles as new carriers for the delivery of plasmid DNA. Journal of Controlled Release 75, 211-224, doi:https://doi.org/10.1016/S0168-3659(01)00397-2 (2001).

82 Khung, Y. L., Bastari, K., Cho, X. L., Yee, W. A. \& Loo, S. C. J. Designing calcium phosphate-based bifunctional nanocapsules with bone-targeting properties. $J$ Nanopart Res 14, doi:ARTN 911 10.1007/s11051-012-0911-8 (2012).

83 Wang, X. et al. Nanocapsules engineered from polyhedral ZIF-8 templates for bone-targeted hydrophobic drug delivery. Biomater Sci 5, 658-662, doi:10.1039/c6bm00915h (2017).

84 Svenson, S. Dendrimers as versatile platform in drug delivery applications. European Journal of Pharmaceutics and Biopharmaceutics 71, 445-462, doi:http://dx.doi.org/10.1016/i.ejpb.2008.09.023 (2009).

85 Ouyang, L., Pan, J. Z., Zhang, Y. \& Guo, L. Synthesis of Second- and ThirdGeneration Asp Oligopeptide Conjugated Dendrimers for Bone-Targeting Drug Delivery. Synthetic Commun 39, 4039-4052, doi:Pii 91593012910.1080/00397910902883660 (2009).

86 Cavero, E., Zablocka, M., Caminade, A. M. \& Majoral, J. P. Design of Bisphosphonate-Terminated Dendrimers. Eur J Org Chem, 2759-2767, doi:10.1002/ ejoc.200901291 (2010).

87 Gu, W. Y., Wu, C. T., Chen, J. Z. \& Xiao, Y. Nanotechnology in the targeted drug delivery for bone diseases and bone regeneration. Int $J$ Nanomed 8, doi:10.2147/Ijn.S44393 (2013).

88 Jimenez-Andrade, J. M. et al. Bone cancer pain. Ann Ny Acad Sci 1198, 173-181, doi:10.1111/j.1749-6632.2009.05429.x (2010).

89 Guise, T. Examining the Metastatic Niche: Targeting the Microenvironment. Semin Oncol 37, S2-S14, doi:10.1053/j.seminoncol.2010.10.007 (2010). 
90 Osasan, S. et al. Osteogenic Sarcoma: A 21st Century Review. Anticancer Res 36, 4391-4398 (2016).

91 Luetke, A., Meyers, P. A., Lewis, I. \& Juergens, H. Osteosarcoma treatment Where do we stand? A state of the art review. Cancer Treatment Reviews 40, 523-532, doi:http://dx.doi.org/10.1016/i.ctrv.2013.11.006 (2014).

92 Low, S. A., Yang, J. \& Kopeček, J. Bone-Targeted Acid-Sensitive Doxorubicin Conjugate Micelles as Potential Osteosarcoma Therapeutics. Bioconjugate Chemistry 25, 2012-2020, doi:10.1021/bc500392x (2014).

93 Seidlin, S. M., Marinelli, L. D. \& Oshry, E. Radioactive iodine therapy; effect on functioning metastases of adenocarcinoma of the thyroid. Journal of the American Medical Association 132, 838-847 (1946).

94 Lewington, V. J. Bone-seeking radionuclides for therapy. J Nucl Med 46 Suppl 1, 38S-47S (2005).

95 Tomblyn, M. The Role of Bone-Seeking Radionuclides in the Palliative Treatment of Patients With Painful Osteoblastic Skeletal Metastases. Cancer Control 19, 137144 (2012).

96 CHAKRABORTY, S. et al. 177Lu labelled polyaminophosphonates as potential agents for bone pain palliation. Nuclear Medicine Communications 23, 67-74 (2002).

97 Zolghadri, S. et al. Production, Quality Control and Biological Evaluation of (166)Ho-PDTMP as a Possible Bone Palliation Agent. Iranian Journal of Basic Medical Sciences 16, 719-725 (2013).

98 Popwell, S. J. et al. Synthesis of polymeric phosphonates for selective delivery of radionuclides to osteosarcoma. Cancer Biother Radiopharm 29, 273-282, doi:10.1089/cbr.2014.1615 (2014).

99 Segal, E. et al. Enhanced anti-tumor activity and safety profile of targeted nanoscaled HPMA copolymer-alendronate-TNP-470 conjugate in the treatment of bone malignances. Biomaterials 32, 4450-4463, doi:10.1016/j.biomaterials.2011.02.059 (2011).

100 Qiao, H. et al. Targeting Osteocytes to Attenuate Early Breast Cancer Bone Metastasis by Theranostic Upconversion Nanoparticles with Responsive Plumbagin Release. ACS Nano 11, 7259-7273, doi:10.1021/acsnano.7b03197 (2017).

101 Lazzarini, L., Lipsky, B. A. \& Mader, J. T. Antibiotic treatment of osteomyelitis: what have we learned from 30 years of clinical trials? Int J Infect Dis 9, 127-138, doi:10.1016/j.ijid.2004.09.009 (2005).

102 Spellberg, B. \& Lipsky, B. A. Systemic antibiotic therapy for chronic osteomyelitis in adults. Clin Infect Dis 54, 393-407, doi:10.1093/cid/cir842 (2012).

103 Gitelis, S. \& Brebach, G. T. The treatment of chronic osteomyelitis with a biodegradable antibiotic-impregnated implant. J Orthop Surg (Hong Kong) 10, 53-60 (2002).

104 Haidar, R., Der Boghossian, A. \& Atiyeh, B. Duration of post-surgical antibiotics in chronic osteomyelitis: empiric or evidence-based? Int J Infect Dis 14, e752-758, doi:10.1016/j.ijid.2010.01.005 (2010).

105 Inzana, J. A., Schwarz, E. M., Kates, S. L. \& Awad, H. A. Biomaterials approaches to treating implant-associated osteomyelitis. Biomaterials 81, 58-71, doi:http://dx.doi.org/10.1016/j.biomaterials.2015.12.012 (2016).

106 Takahashi, T. et al. Bone-targeting of quinolones conjugated with an acidic oligopeptide. Pharm Res 25, 2881-2888, doi:10.1007/s11095-008-9605-4 (2008). 
107 Burge, R. et al. Incidence and economic burden of osteoporosis-related fractures in the United States, 2005-2025. J Bone Miner Res 22, 465-475, doi:10.1359/jbmr.061113 (2007).

108 Jeremiah, M. P., Unwin, B. K., Greenawald, M. H. \& Casiano, V. E. Diagnosis and Management of Osteoporosis. Am Fam Physician 92, 261-268 (2015).

109 Riek, A. E. \& Towler, D. A. The Pharmacological Management of Osteoporosis. Missouri medicine 108, 118-123 (2011).

110 Andreassen, K. V. et al. Prolonged calcitonin receptor signaling by salmon, but not human calcitonin, reveals ligand bias. Plos One 9, e92042, doi:10.1371/journal.pone.0092042 (2014).

111 Cawthray, J., Wasan, E. \& Wasan, K. Bone-seeking agents for the treatment of bone disorders. Drug Deliv Transl Res 7, 466-481, doi:10.1007/s13346-017-0394-3 (2017).

112 Lee, K. C. et al. Intranasal delivery of PEGylated salmon calcitonins: hypocalcemic effects in rats. Calcif Tissue Int 73, 545-549, doi:10.1007/s00223-002-0034-9 (2003).

113 Bhandari, K. H., Newa, M., Uludag, H. \& Doschak, M. R. Synthesis, characterization and in vitro evaluation of a bone targeting delivery system for salmon Calcitonin. International Journal of Pharmaceutics 394, 26-34, doi:https://doi.org/10.1016/i.ijpharm.2010.04.015 (2010). 


\section{Chapter 3}

\section{Development of bone seeker-functionalized microspheres as a targeted local antibiotic delivery system for bone infections}

S.G. Rotman ${ }^{1,2}$, K. Thompson ${ }^{1}$, D.W. Grijpma ${ }^{2}$, R.G. Richards ${ }^{1}$, T.F. Moriarty $^{1}$, D. Eglin ${ }^{1}$ and O. Guillaume ${ }^{1}$

${ }^{1}$ AO Research Institute Davos, Davos Platz, Switzerland

${ }^{2}$ Department of Biomaterials Science and Technology, Faculty of Science and Technology, University of Twente, Enschede, The Netherlands 


\section{Abstract}

Bone infections are challenging to treat due to limited capability of systemic antibiotics to accumulate at the bone site. To enhance therapeutic action, systemic treatments are commonly combined with local antibiotic-loaded materials. Nevertheless, such drug carriers have undesirable properties, including inappropriate antibiotic release profiles and non-biodegradability. To alleviate such limitations, we aim to develop a drug delivery system for local administration that can interact strongly with bone mineral, releasing antibiotics at the infected bone site. Biodegradable polyesters (poly $(\epsilon-$ caprolactone) or poly(D,L-lactic acid)) were selected to fabricate antibiotic loaded microspheres by oil in water emulsion. Antibiotic release and antimicrobial effects on S. aureus were of a sustained nature. Microsphere bone affinity was increased by functionalizing the bisphosphonate drug alendronate to the microsphere surface using carbodiimide chemistry. Effect of bone targeting microspheres on bone homeostasis was tested by looking at the resorption potential of osteoclasts exposed to the developed microspheres. In vitro, the antibiotic release profile from the microspheres was shown to be dependent on the polymer used and the microsphere preparation method. Mineral binding assays revealed that microsphere surface modification with alendronate significantly enhanced interaction with bone-like materials. Additionally, alendronate functionalized microspheres did not differentially affect osteoclast mineral resorption in vitro, compared to non-functionalized microspheres. We report the development and characterization of a drug delivery system which can release antibiotics in a sustained manner. Surface-grafted alendronate groups enhanced bone affinity of the microsphere construct, resulting in a bone 
targeting drug delivery system. The drug delivery system presented can be loaded with hydrophobic antibiotics, representing a potential, versatile and biodegradable candidate to locally treat bone infection.

\section{Introduction}

Infection of bone, also known as osteomyelitis $(\mathrm{OM})$, is challenging to treat by systemic antibiotic administration due to impaired vascularity of infected bone tissue [1], limited capacity of bone tissue to retain drugs for long periods of time and due to a poor ability of many common antibiotics to effectively penetrate infected bone [2]. All of these factors can result in an insufficient local antibiotic concentration that is then unable to clear the infection successfully [3]. Consequently, the current consensus for OM treatment is an initial debridement of infected tissues followed by the synergetic use of systemically administered antibiotics and local drug delivery systems (DDS) that can locally release antibiotics over an extended period of time. However, combination of therapy is not always successful in treating chronic OM, as reinfection rates are still relatively high and can reach approximately $30 \%$ for patients with complicated open bone fractures [4]. One of the main reasons for reinfection originates in the inability of the antibiotic to reach a sufficiently high concentration within infected bone tissue, where viable bacteria may remain.

Gentamicin sulfate (GM) is a broad-spectrum antibiotic, used to treat blood, skin-, lung-, joint- and bone-infections. In addition, due to GM heat stability, it is one of the most frequently used antibiotics for exothermically setting Poly(methyl methacrylate) (PMMA) bone cements [5]. GEN loaded PMMA cements are frequently implanted as a local DDS, either as a prophylactic approach or as a treatment for bone infections since the 1970s. 
However, a particular disadvantage of PMMA is its non-degradability and incomplete drug release [6], necessitating a further surgical procedure to remove PMMA cements. Such limitations motivate the search for an alternative degradable and more versatile material to be used for local antibiotic delivery strategies.

Loading GM in degradable DDS has also been proposed, but it often results in a fast elution due to its high solubility in aqueous solutions and low affinity to hydrophobic matrices [7]. Being a salt, GM can undergo hydrophobic ion pairing (HIP) to impose hydrophobic properties to the drug. By substituting the sulfate ion of GM with other anions such as crobefate [8] or pamoate ions (gentamicin embonate), hydrophobicity and matrix affinity increases. We have previously synthesized a hydrophobic GM analogue, gentamicindioctyl sulfosuccinate (GM-AOT) following established protocols $[9,10]$. Water solubility of GM-AOT $(0.43 \mathrm{mg} / \mathrm{mL})$ is dramatically reduced compared to GM $(100 \mathrm{mg} / \mathrm{mL})$, but it maintains antimicrobial activity against Staphylococcus aureus and Staphylococcus epidermidis, comparable to GM [9].

Frequently applied materials for DDS development are biodegradable polyesters such as poly(D,L-lactic acid) (PDLLA) [11], poly(lactic-co-glycolic acid) (PLGA) [12] and poly( $\varepsilon$-caprolactone) (PCL) [13]. The respective monomers and their isomeric composition dictate their degradation behavior. Poly(lactic acid) with only L-isomers (PLLA) is more crystalline compared to poly(lactic acid) with both L- and D-isomers (PDLLA), making PDLLA more susceptible to hydrolysis. PCL does not have optical isomers and can be considered a hydrophobic semi-crystalline polymer. This results in slower in vivo degradation compared to the other members of the polyester 
family like PLGA [14]. This information highlights the importance of material choice in designing a DDS.

The majority of DDS previously reported do not exhibit intrinsic affinity to bone, which limits their clinical potential for drug delivery to bone. However, there are many molecules available that exhibit affinity to bone, which can be used to generate bone targeting DDS. The use of so called 'bone seeking' or ‘bone binding` agents combined with DDS have brought promising preclinical and clinical solutions to treat bone-related disorders and were recently reviewed [15]. The inorganic matrix of bone, consisting of hydroxyapatite (HAP) crystals, is often targeted by these bone binding molecules. As an example, bisphosphonates (BPs) are known for their rapid and strong binding to calcium ions in HAP via chelation, facilitated by their two phosphonate groups [16]. The BP alendronate (ALN) is frequently incorporated into drug delivery systems [17-19], as its side chain contains a reactive primary amine that is available for conjugation chemistry.

In this work, we synthesized and characterized a degradable microsphere drug delivery system, loaded with GM-AOT antibiotic in vitro. We assessed binding capabilities of ALN functionalized PCL microspheres to bone and in vitro efficacy of the antibiotic released from the DDS. Drug delivery to bone has been undertaken in the field of Osteosarcoma (e.g. by delivery of Doxorubicin [20]) and Osteoporosis (e.g. by delivery of Simvastatin [21]), but often these delivery systems don't show inherent affinity to bone. To the best of our knowledge, such DDS for antibiotics have not been reported in the field of bone infection. Compared to laborious (amphiphilic) co-polymer strategies reported in the literature [22], the presented DDS can be fabricated from common PCL with minimal post-treatment procedures to enhance its 
affinity to HAP. Such active DDS represents a promising, versatile and upscalable candidate to improve the local treatment of bone infection.

\section{Materials and Methods}

\section{Materials}

PCL $\left(\mathrm{M}_{\mathrm{w}}=80.000 \mathrm{~g} \cdot \mathrm{mol}^{-1}\right.$, polydispersity index $\left.<2\right)$, boric acid, pyrene, ophthaldialdehyde, methanol, hydrochloric acid $(\mathrm{HCl}), \mathrm{N}$ hydroxysuccinimide (NHS), dioctyl sodium sulfosuccinate sodium salt (AOT), silver nitrate, sodium thiosulfate, gentamicin sulfate salt (GM), phosphate buffered saline (PBS), tartrate-resistant acid phosphatase (TRAP) staining kit and alendronate sodium were purchased from Sigma Aldrich (Steinheim, Germany). PDLLA $\left(\mathrm{M}_{\mathrm{w}}=215.000 \mathrm{~g} \cdot \mathrm{mol}^{-1}, 80 \% \mathrm{~L}, 20 \% \mathrm{D}: \mathrm{L}\right)$ was bought from Corbion Purac (Gorinchem, the Netherlands). Poly(vinyl alcohol) (PVA, $\left.\mathrm{M}_{\mathrm{w}}=31.000 \mathrm{~g} \cdot \mathrm{mol}^{-1}\right)$, sodium hydroxide $(\mathrm{NaOH})$, dichloromethane $\left(\mathrm{CH}_{2} \mathrm{Cl}_{2}\right)$, dimethyl sulfoxide (DMSO) and 2-propanol was bought from Carl Roth (Karlsruhe, Germany). 1-Ethyl-3-(3dimethylaminopropyl)carbodiimide (EDC) was purchased from Fluka (Buchs, Switzerland). (5-((2-Aminoethyl)amino)naphthalene-1-sulfonic acid) (EDANS) was bought from Anaspec (Seraing, Belgium). Tryptone soy agar (TSA) was purchased from Oxoid AG (Basel, Switzerland). Alpha minimum essential media, fetal bovine serum and penicillin/streptomycin were bought from Gibco (Zug, Switzerland). Clinically isolated Staphylococcus aureus JAR (CCOS, Wädenswill, Switzerland) strain was used as the bacterial pathogen throughout the study. Receptor activator of nuclear factor kappa-B ligand (RANKL) and macrophage-colony stimulating factor (MCSF) growth factors were purchased from R\&D Systems (Abingdon, UK). Osteoassay plates were acquired from Corning (Amsterdam, the Netherlands). Ultra- 
pure milliQ water $(18.2 \mathrm{M} \Omega \cdot \mathrm{cm})$ was used during all experiments. Methacrylated-Poly(trimethylene carbonate) scaffolds containing $40 \mathrm{wt} \%$ dispersed nano-hydroxyapatite were kindly provided from collaborators at the University of Twente (Enschede, the Netherlands) and were prepared as previously described [23].

\section{GM-AOT synthesis by hydrophobic ion pairing}

GM-AOT was synthesized as described by ter Boo et al. [9]. Briefly, equal volumes of $1.25 \% \mathrm{w} / \mathrm{v}$ dioctyl sodium sulfosuccinate (AOT) in $\mathrm{CH}_{2} \mathrm{Cl}_{2}$ and $0.40 \% \mathrm{w} / \mathrm{v} \mathrm{GM}$ in buffer $\left(10 \mathrm{mM}\right.$ sodium acetate, $\mathrm{KCl}$ and $\mathrm{CaCl}_{2}$ at $\mathrm{pH}=$ 5 ) were mixed by vigorous stirring for 3 hours. The two phases separated for 30 minutes and GM-AOT was isolated from the dichloromethane by evaporation of the solvent. Fourier transform infrared spectroscopy (FTIR) characterization was performed to validate the successful pairing of AOT with GM. The water solubility of GM-AOT was determined by complexation of saturated aqueous solutions with o-phthaldialdehyde reagent in a colorimetric assay, at a wavelength of $332 \mathrm{~nm}$ using a spectrophotometer (MultiskanGo, Thermo Scientific) [24].

\section{Fabrication of PCL and PDLLA microspheres loaded with GM-AOT or Pyrene}

PCL or PDLLA (500 mg) was dissolved in $5 \mathrm{~mL} \mathrm{CH}_{2} \mathrm{Cl}_{2}$. As encapsulated compounds, 25\% w/w GM-AOT or 10\% w/w Pyrene (used as a probe for a fluorimetic assay) relative to the weight of the polymer, was added to the polymers in solution. PVA solution $(100 \mathrm{~mL}, 1 \% \mathrm{w} / \mathrm{v})$ acted as the aqueous phase during the emulsion process. Emulsions were prepared by probe sonication (Bandelin Sonopuls GM70, 3 bursts of 10 seconds, 700W) or by 
ultra-turrax homogenization (Silverson LM5, 10 minutes at $10.000 \mathrm{rpm}$ ). Under moderate stirring, the organic solvent was evaporated over 4 hours at room temperature, resulting in solidified microspheres. The microspheres were collected by centrifugation at $68.000 \mathrm{~g}$ with an ultracentrifuge (Sorvall Discovery $90 \mathrm{SE}$ ), and were washed and resuspended twice with milliQ water to remove the excess of the PVA surfactant. The microspheres were then lyophilized and stored at $4{ }^{\circ} \mathrm{C}$ until utilization. To determine drug loading, GM-AOT loaded microspheres were placed in $1 \mathrm{M} \mathrm{NaOH}$ to hydrolyze the microspheres and GM-AOT was subsequently quantified after complexation with o-phthaldialdehyde reagent as previously described [24]. The encapsulation efficiency (EE\%) of the microspheres is calculated following equation 1:

$E E \%=\left(G M-A O T_{\text {Encapsulated }} / G M-A O T_{\text {Initial }}\right) \cdot 100 \%$

With GM-AOT $\mathrm{T}_{\text {Encapsulated }}$ as the amount of GM-AOT spectrophotometrically quantified and GM-AOT Initial as the amount of GM-AOT added to the emulsion system. To ensure batch-to-batch reproducibility another batch was fabricated under identical circumstances.

\section{Release profile of GM-AOT from PCL and PDLLA microspheres}

$10 \mathrm{mg}$ of GM-AOT loaded microspheres $(\mathrm{n}=6)$ were incubated in $1 \mathrm{~mL}$ of PBS at $37^{\circ} \mathrm{C}$ under mild shaking $(30 \mathrm{rpm})$. At each time point (2 hours, 6 hours, 1 day, 2 days, 3 days, 6 days, 12 days and 14 days), the samples were centrifuged at $16.000 \mathrm{~g}$ for 10 minutes (Eppendorf centrifuge 5415D) and the supernatants were carefully removed. At each timepoint, the PBS was replaced with $1 \mathrm{~mL}$ of fresh PBS. Supernatants were assessed for their GMAOT content, as previously described. 


\section{Surface functionalization with EDANS or ALN}

The functionalization of the polyester-based microspheres required initial surface hydrolysis to increase the fraction of carboxylic groups. The lyophilized microspheres were dispersed in $0.1 \mathrm{M} \mathrm{NaOH}$ solution (10 $\mathrm{mg} / \mathrm{mL}$ ) and the microspheres' surface were saponified (alkaline induced hydrolysis) for 30, 60 or 120 minutes. To terminate the saponification process, $2 \mathrm{~mL}$ of $1 \mathrm{M} \mathrm{HCl}$ was added to facilitate the formation of carboxyl groups $(-\mathrm{COOH})$ from the formed $\mathrm{COO}^{-} \mathrm{Na}^{+}$ionic chain ends. The microspheres were collected by centrifugation for 15 minutes at $68.000 \mathrm{~g}$ and washed in milliQ water. The saponified microspheres were then dispersed in $10 \mathrm{~mL}$ 2-(N-morpholino)ethanesulfonic acid (MES)-buffer $(\mathrm{pH}=5.5)$ containing EDC (50 mg, $0.26 \mathrm{mmol})$ and NHS (50 mg, $0.43 \mathrm{mmol})$. The microspheres underwent carboxyl activation for 30,60 or 120 minutes and were collected by centrifugation for 15 minutes at $68.000 \mathrm{~g}$, followed by washing in milliQ water. To assess the ability of compounds with primary amine groups to be conjugated to the microspheres by EDC/NHS chemistry, the microspheres were dispersed in $2 \mathrm{~mL}$ of aqueous solution of fluorescent EDANS $(250 \mu \mathrm{g} / \mathrm{mL})$ and conjugation was allowed to proceed for 60 minutes under mild stirring at room temperature. The relative amount of surface grafted EDANS was measured using a fluorescence plate reader (Viktor ${ }^{3}$, Perkin Elmer) at $461 \mathrm{~nm}$. For ALN conjugation, 30 minutes of saponification and 30 minutes of EDC/NHS activation were used based on satisfactory surface conjugation of EDANS.

To monitor the effect of $\mathrm{NaOH}$ exposure to the molecular weight of the PCL polymers, Gel permeation chromatography (GPC) was performed. PCL microspheres and saponificated PCL microspheres (30 minutes) were 
dissolved in chloroform $(1 \mathrm{mg} / \mathrm{mL})$ and injected into the GPC system (Agilent 6850).

\section{PCL-ALN affinity to bone-like material}

Pyrene was chosen as an internalized fluorophore to assess the microparticle affinity to HAP. Pyrene encapsulation in PCL microspheres and surface functionalization with ALN was performed as described earlier. To perform the HAP binding assay, a bone-like methacrylated-poly(trimethylene carbonate) scaffold containing 40\% w/w of dispersed nano-HAP powder, made by stereolithography, was used as a macroscopic binding substrate. The fabrication methods of these scaffold materials can be found in previous publications $[23,25]$. The fabricated nano-HAP scaffolds were incubated for two hours in a $500 \mu \mathrm{g} / \mathrm{mL}$ PCL-ALN or PCL microsphere dispersion as control. After incubation, the scaffolds were thoroughly washed with distilled water to remove non-chelated microspheres from the surface. Fluorescence from the scaffolds incubated with pyrene-loaded PCL and PCL-ALN microspheres was measured on a Viktor ${ }^{3}$ plate reader (Perkin Elmer) with $\lambda_{\mathrm{ex}} / \lambda_{\mathrm{em}} \sim 353 \mathrm{~nm} / 483 \mathrm{~nm}$.

\section{Inhibitory effect of ALN-functionalized microspheres on osteoclasts}

Monocyte/macrophage-lineage osteoclast precursors were isolated from the bone marrow of female, balb/c mice, $n=2$, according to guidelines of the cantonal authorities (Tierversuchskommission Graubünden) and were kindly donated by the Swiss Institute of Allergy and Asthma Research (TVB number: 27653; Cantonal number: GR 2016_02F). The osteoclast precursors were cultured in standard culture conditions $\left(37^{\circ} \mathrm{C}, 5 \% \mathrm{CO}_{2}\right)$ with $\alpha \mathrm{MEM}$ supplemented with $10 \% \mathrm{v} / \mathrm{v}$ fetal bovine serum, $1 \% \mathrm{v} / \mathrm{v}$ 
penicillin/streptomycin and $50 \mathrm{ng} / \mathrm{mL}$ of recombinant murine macrophagecolony stimulating factor (M-CSF). After 5-7 days, $10 \mathrm{ng} / \mathrm{mL}$ of recombinant murine RANKL was added to the culture medium to support the fusion of osteoclast precursors into osteoclasts. After 48 hours, the osteoclasts were trypsinized and seeded on Osteoassay 96 well at a density of $10^{4}$ cells/well. After allowing the cells to attach to the substrate for 4 hours, $10 \mu \mathrm{M}$ ALN solution in PBS, or $1.7 \mathrm{mg}$ of PCL and PCL-ALN microspheres, were added to the respective wells. This quantity of PCL-ALN particles corresponded to $10 \mu \mathrm{M}$ of surface grafted ALN, as established by spectrophotometrical analysis of EDANS grafting. After 72 hours the cells and microspheres were washed twice with PBS and the content of the wells was fixed using 4\% formaldehyde solution for 15 minutes at room temperature. The osteoclasts were stained with a TRAP staining kit prior to brightfield microscopy imaging. After assessing the osteoclast morphology, the cells were removed by using $10 \% \mathrm{v} / \mathrm{v}$ bleach, and the HAP substrate was stained with a Von Kossa silver nitrate stain to visualize HAP resorption and imaged using an inverse light microscopy (Zeiss Axiovert.A1). The area of stained mineral was quantified after binarization of the image using AxioVision4 software (Zeiss).

\section{In vitro antimicrobial assessment}

The minimal inhibitory concentration (MIC) and minimal bactericidal concentration (MBC) was determined by supplementing GM-AOT loaded PCL, GM-AOT or GM in the Staphylococcus aureus (S. aureus) CAMH culture broth. Antibiotic solutions and particle dispersions in CAMH broth were made by $1: 1$ dilution to achieve the range of $500 \mathrm{ug} / \mathrm{mL}$ to $0.49 \mathrm{ug} / \mathrm{mL}$ (GM and GM-AOT) or $5000 \mathrm{ug} / \mathrm{mL}$ to $5 \mathrm{ug} / \mathrm{mL}$ (PCL/GM-AOT microspheres). 
GM-AOT was initially dissolved in DMSO and was subsequently 1:9 diluted with CAMH broth to reach comparable concentrations with GM. The final content of the wells of a 96-well plate was $100 \mu \mathrm{L} \mathrm{S}$. aureus inoculum $\left(10^{5}\right.$ $\mathrm{CFU} / \mathrm{mL}$ ) and $100 \mu \mathrm{L}$ antibiotic solution or particle dispersion. The cultures were incubated for 24 hours at $37^{\circ} \mathrm{C}$ and $5 \% \mathrm{CO}_{2}$, after which $100 \mu \mathrm{L}$ per condition was plated on a TSA plate to quantify the CFU. All conditions were measured in triplicate.

Time dependent inhibitory properties of the GM-AOT loaded PCL microspheres were assessed with zone of inhibition (ZOI) experiments. $S$. aureus were cultured on TSA plates in standard culture conditions of $37^{\circ} \mathrm{C}$ and $5 \% \mathrm{CO}_{2}$. In the center of the TSA plate, $10 \mathrm{mg}$ of GM-AOT loaded microspheres were deposited on a sterile wafer (Sensi-disc, BD Biosciences) and wetted with $20 \mu \mathrm{L}$ of PBS. The wafer was transferred daily to a freshly plated $S$. aureus culture and incubated for 24 hours. The radius of the $S$. aureus-free zone around the antimicrobial sample was measured daily. A 50 $\mathrm{mg} / \mathrm{mL}$ GM-AOT solution in DMSO in a volume of $20 \mu \mathrm{L}$ was used to mimic a single systemic drug administration. Two clinical reference DDS were assessed as positive controls for their ability to inhibit $S$. aureus growth:

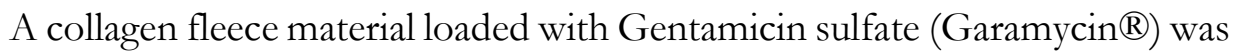
cut in the size of $1 \mathrm{~cm}^{2}$ (containing approximately $1.3 \mathrm{mg}$ of Gentamicin sulfate); and $110 \pm 5 \mathrm{mg}$ of PMMA bone cement (Copal ${ }^{\circledR}$, containing approximately $1.3 \mathrm{mg}$ of Gentamicin sulfate and $5.2 \mathrm{mg}$ of Vancomycin).

\section{Scanning electron microscopy imaging}

Lyophilized microsphere samples before and after surface conjugation with ALN were dispersed in ethanol and $40 \mu \mathrm{L}$ of the microsphere dispersion was pipetted on SEM pin stub specimen mounts covered with a conductive 
adhesive layer. The ethanol was evaporated at room temperature. The samples were then sputter-coated with $\mathrm{Au} / \mathrm{Pd}$ with a layer thickness of 10 $\mathrm{nm}$ and a deposit rate of $0.05 \mathrm{~nm} / \mathrm{s}$. SEM imaging was performed on a field emission scanning electron microscope (S-4700, Hitachi). The working distance of the SEM was maintained at approximately $8 \mathrm{~mm}$ and an acceleration beam voltage of $1.5 \mathrm{keV}$ was used. For microsphere size analysis, the diameter of a minimum of 100 PCL or PDLLA microspheres were measured to achieve a reliable and representable size average.

\section{Statistical Analysis}

Statistical significance was assessed using GraphPad Prism software. Oneway ANOVA with Bonferroni post-test was applied on multivariable data that was tested for normality by Q-Q plot interpretation. When data with a single variable was analyzed, Student's unpaired T-test with a Tukey post-test was used with a confidence interval of $95 \%$.

\section{Results}

\section{Synthesis of GM-AOT}

Figure 3.1 shows the FTIR spectra of GM, AOT, GM-AOT and a physical blend of GM and AOT. A characteristic peak at the wavenumber $1740 \mathrm{~cm}^{-1}$ is observed for AOT, GM-AOT and the physical blend, while a peak related to $\mathrm{GM}$ at $1640 \mathrm{~cm}^{-1}$ is found for GM, GM-AOT and the physical blend. These FTIR spectra confirm successful hydrophobic ion pairing between AOT and GM. 


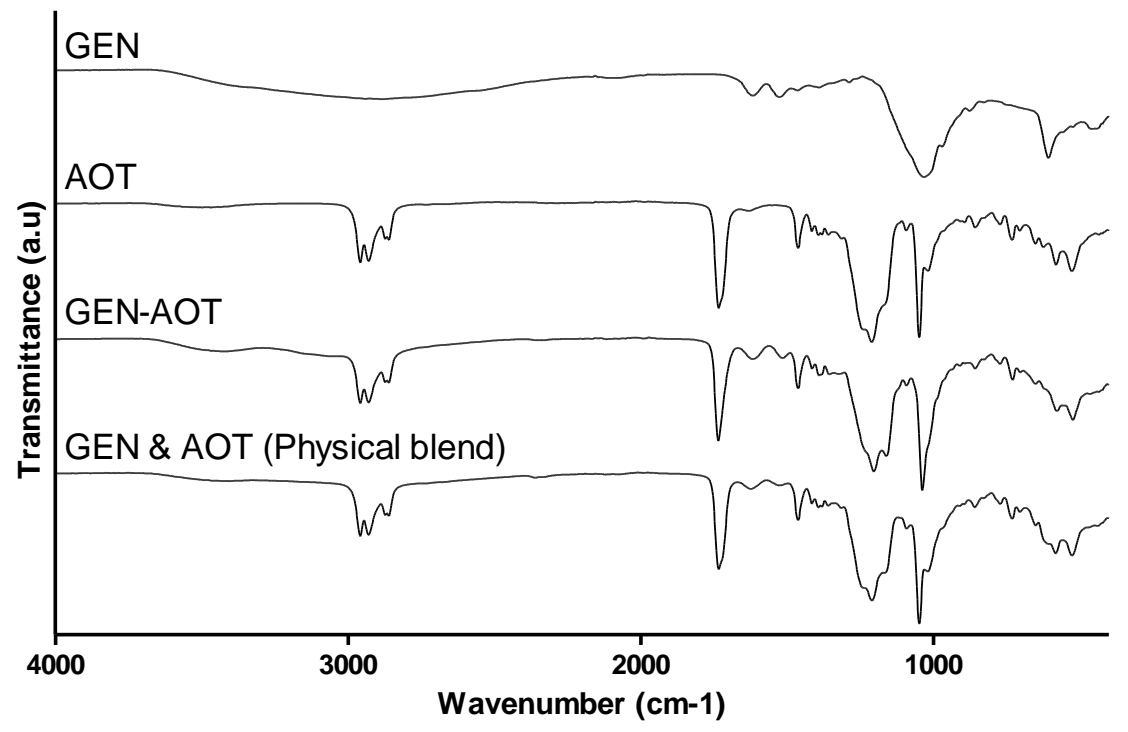

Figure 3.1 - FTIR characterization of GM-AOT and its individual constituents. GMAOT expresses the combined spectrum of GM and AOT. The physical blend of GM and AOT resulted in a similar spectrum as the GM-AOT salt that was fabricated by hydrophobic ion pairing.

Nature of the matrix and fabrication method influence microsphere size and drug encapsulation

The size distribution, average microsphere diameter $(\varnothing)$, the EE\% of GMAOT, and surface morphology of the four different microsphere formulations are shown in Figure 3.2. The two variables assessed were the nature of the polymeric matrix (PCL or PDLLA) and the method of emulsification (probe sonication (SON) or high-speed mechanical mixing by ultra-turrax (HSM)). 
A.
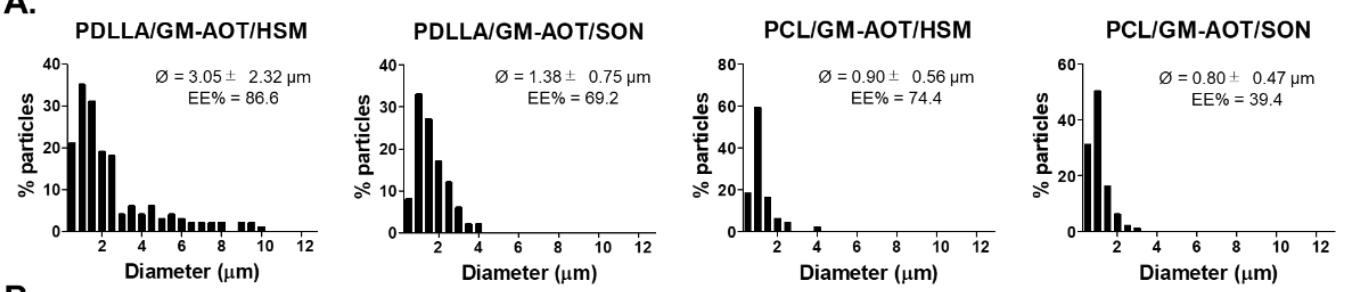

B.
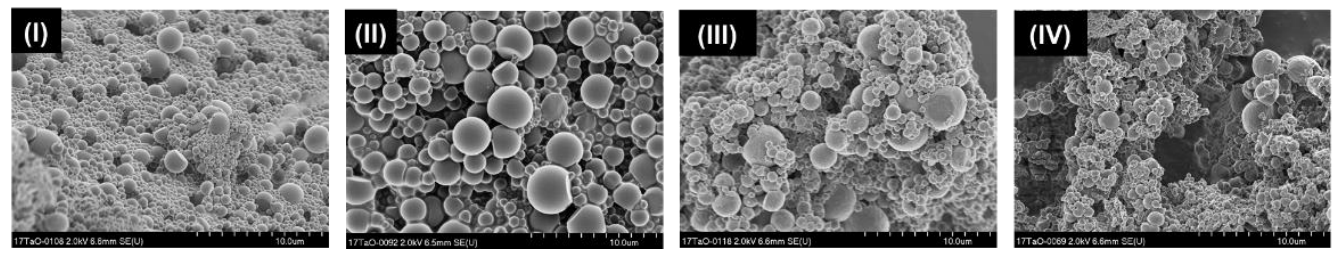

Figure 3.2 - Microsphere size and morphology characterization.

A. Size distribution histogram (interval $=0.5 \mu \mathrm{m}$ ) of all formulations of microparticles. $(n=100)$. The average microsphere diameter and standard deviation (Ø) and the encapsulation efficiency of the respective antibiotics (EE\%) are noted on every histogram.

B. Representative SEM images of PDLLA/GM-AOT/HSM (I), PDLLA/GMAOT/SON (II), PCL/GMAOT/HSM (III) and PCL/GM-AOT/SON (IV).

Figure 3.2A shows that the use of PDLLA resulted in right sided skewness in size distribution which in turn resulted in higher $\varnothing$ values, in comparison to PCL microspheres. The method of emulsification also impacted on their diameter. The use of HSM resulted in a wider size distribution compared emulsions made by SON. EE\% of GM-AOT as high as 86.6\% in PDLLA matrix and $74.4 \%$ in PCL matrix were achieved. While SON resulted in a reduced $\mathrm{EE} \%$ of GM-AOT, it was also responsible for a smaller and more homogenous particle size distribution. The use of PDLLA compared to PCL was systematically responsible to an increase of the $\mathrm{EE} \%$. This could be attributed to the higher molecular weight of PDLLA, larger size of PDLLA microspheres and physio-chemical property differences of the polymers used. The morphology of all microsphere formulations observed using SEM (Figure 3.2B) revealed that the surfaces are smooth without presence of 
pores. As will be explained in further sections of this results section, $\mathrm{PCL} / \mathrm{GM}-\mathrm{AOT} / \mathrm{SON}$ microspheres were chosen for further functionalization and characterization. Reproducibility of microsphere fabrication with this methodology was established with further batches showing similar size distributions $(0.85 \pm 0.46 \mu \mathrm{m}$ versus $0.80 \pm 0.47 \mu \mathrm{m})$.

\section{GM-AOT is diffusing continuously from polyester microspheres}

PCL and PDLLA microspheres loaded with GM-AOT are characterized by a sustained release kinetic (Figure 3.3A). The GM-AOT's extremely hydrophobic properties (GM-AOT solubility in water $\approx 0.43 \mathrm{mg} / \mathrm{mL}$ ) prevents burst-style releases. Hydrophobic interactions between GM-AOT and the polymeric matrix is reasoned to result in a more homogenous distribution of the drug in the microspheres. A faster drug release from PCL microspheres is observed compared to PDLLA microspheres. The fabrication method also influenced the drug release kinetics, primarily due to the resulting differences in microsphere size. For example, the PDLLA/GM-AOT/HSM formulation resulted in the largest microsphere size and $\mathrm{EE} \%$ (Fig 3.2), while its release kinetics are the slowest among all tested formulations. The formulation PCL/GM-AOT/SON had the smallest average microsphere size and underwent the fastest release of antibiotic. This correlates with the hypothesis regarding the enrichment of the drugs at the periphery of the microsphere of crystalline materials like PCL. For a similar weight (i.e. the release experiment was performed on 10 $\mathrm{mg}$ ), the smallest microspheres have the largest surface area and thus the fastest diffusion-based release of GM-AOT. 
A.

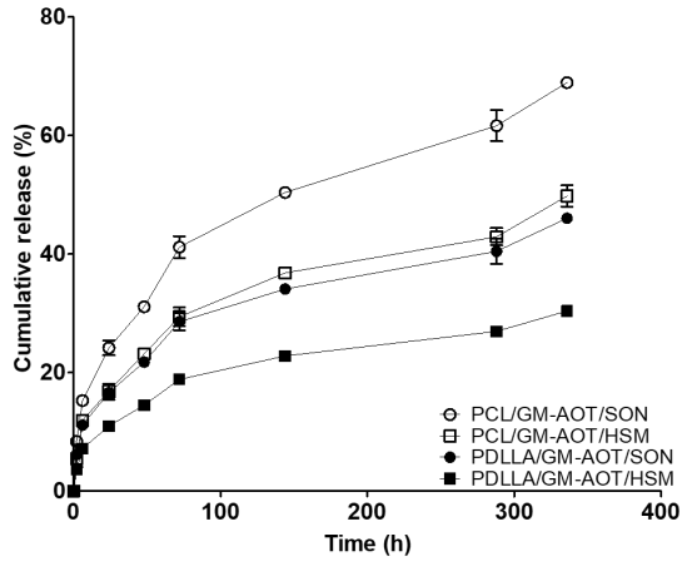

B.

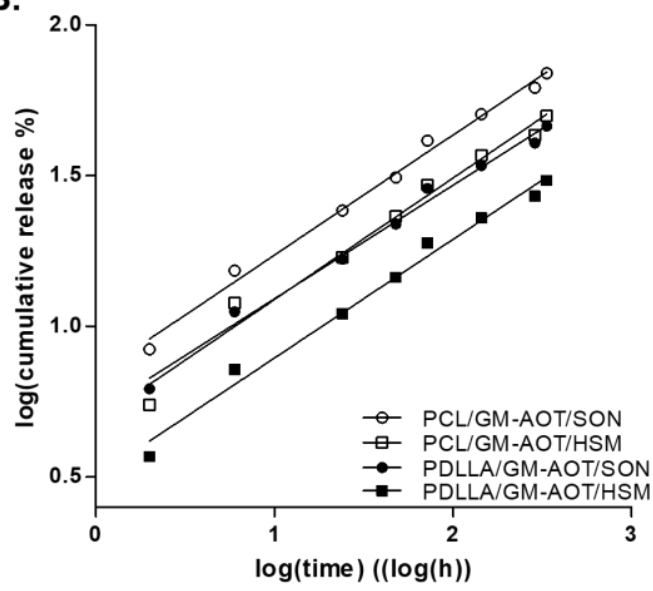

Figure 3.3 - In vitro release profile of GM-AOT from all microsphere formulations $(n=6)$.

A. GM-AOT release profiles from both PCL and PDLLA microspheres. Sustained release of the drug was established after a short burst release in the first 24 hours.

B. GM-AOT release remodeled to fit the Korsmayer-Peppas model for sustained drug release. The trendline fits with high fidelity to the data. Linear trendlines with R2 $>0.983$ for all groups was achieved.

A conventional model used to characterize sustained drug release is the Korsmeyer-Peppas model which is defined by equation (eq. 2)

$$
M_{t} / M_{\infty}=K_{K P} t^{n}
$$

In which $M_{t} / M_{\infty}$ is the cumulative drug released at time $t, K_{K P}$ is the release constant and the $n$ value is used to characterize the type of drug release of the tested system. In Figure 3.3B, the data of the GM-AOT release study was logarithmically manipulated (from $t^{n}$ to $n \log _{10}(t)$ and $M_{t} / M_{\infty}$ to $\left.\log _{10}\left(M_{t} / M_{\infty}\right)\right)$ and averaged, according to the Korsmeyer-Peppas model. When the manipulated data was plotted on a $\log _{10}\left(M_{t} / M_{\infty}\right)$ versus $\log _{10}(t)$ axis system, linear trendlines with slope $n$ fit the experimental data with high precision $\left(\mathrm{R}^{2}>0.983\right)$. Values of $n$ were between $0.31 \pm 0.01$ (PLA/GM- 
AOT/HSM) and 0.40 \pm 0.02 (PCL/GM-AOT/SON), indicating a purely diffusion-based release of drug with negligible effects of polymer erosion (Table 3.1).

Table 3.1 - Parameters of the release kinetics of GM-AOT, modeled according to the Korsmeyer-Peppas model. The data is formed from triplicate data whose curves was fitted for each replicate, establishing the reported SD values.

\begin{tabular}{lcc}
\hline Polymer / Drug / Method & \multicolumn{2}{c}{ Korsmeyer-Peppas model } \\
& $\mathbf{n}$ & $\mathbf{R}^{\mathbf{2}}$ \\
\hline PCL / GM-AOT / SON & $0.40 \pm 0.02$ & $0.988 \pm 0.002$ \\
PCL / GM-AOT / HSM & $0.33 \pm 0.00$ & $0.983 \pm 0.005$ \\
PDLLA / GM-AOT / SON & $0.33 \pm 0.01$ & $0.987 \pm 0.001$ \\
PDLLA / GM-AOT / HSM & $0.31 \pm 0.01$ & $0.984 \pm 0.003$ \\
\hline
\end{tabular}

Due to the higher drug loading capability and the more sustained release of the drug load, PCL microspheres loaded with GM-AOT and fabricated by probe sonication induced $\mathrm{O} / \mathrm{W}$ emulsions $(\mathrm{SON}$ ) were selected for further development into a bone targeting DDS.

\section{Surface functionalization with ALN optimized for EDC/NHS conjugation}

Carboxyl groups on the PCL microsphere surface, necessary for EDC/NHS conjugation, were created by alkaline hydrolysis. The FTIR spectrum in Figure 3.4A, shows the shaded peaks that were used to calculate the C$\mathrm{O} / \mathrm{CH}_{2}$ peak area ratios, which are presented Figure 3.4B. The intensity of $\mathrm{CH}_{2}$ peaks remains stable after saponification, while a higher presence of OC-O peaks is to be expected. Without exposure to $\mathrm{NaOH}$, the C-O/ $\mathrm{CH}_{2}$ ratio is approximately $1.45 \pm 0.02$, while the ratio increases significantly (unpaired t-test $\mathrm{p}=0.017$ ) to $1.61 \pm 0.05$ after 120 minutes of $0.1 \mathrm{M} \mathrm{NaOH}$ 
exposure. GPC analysis, before and after exposure of PCL microspheres to $0.1 \mathrm{M} \mathrm{NaOH}$ revealed that the average polymer degradation of the microspheres was limited. The molecular weight parameters $\left(M_{w}, M_{n}\right.$ and PDI) are listed in Table 3.2. 

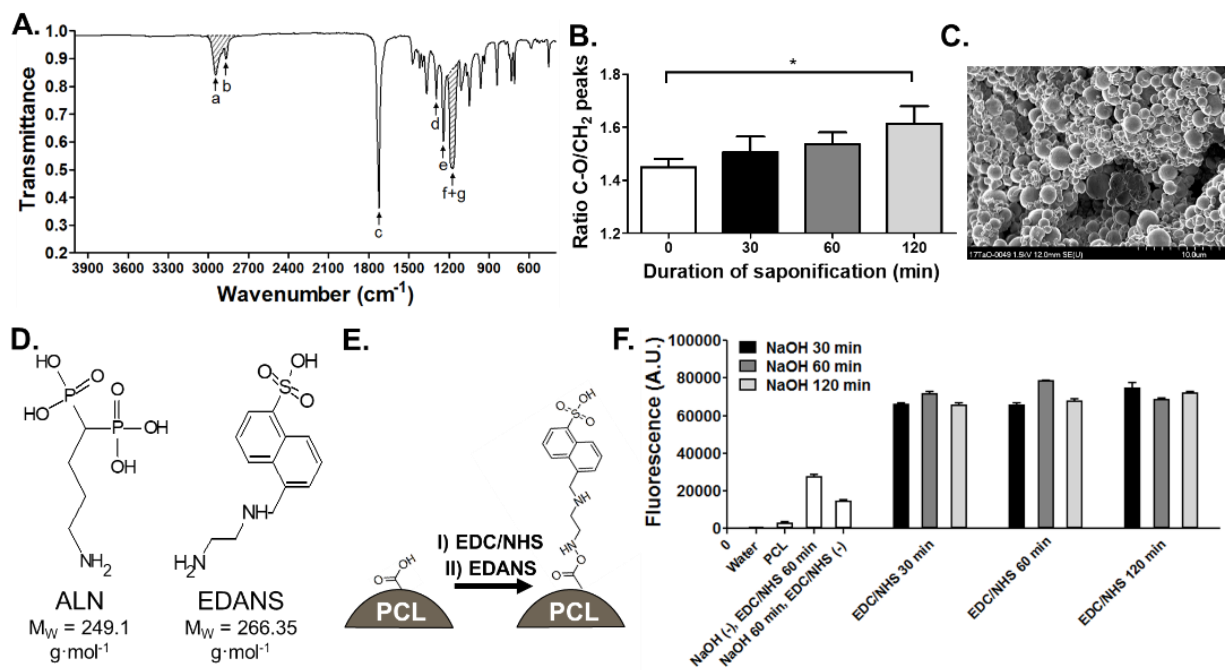

Figure 3.4 - Surface treatment with $\mathrm{NaOH}$ results in an increased presence in carboxylic acid chain ends on the surface of the PCL microspheres, allowing better conjugation with EDANS, a fluorescent model molecule for Alendronate.

A. FTIR spectra of PCL. The labeled peaks represent: a. asymmetric CH2 stretching, b. symmetric $\mathrm{CH} 2$ stretching, c. carbonyl stretching, d. C-O and C-C stretching in the crystalline phase, e. asymmetric COC stretching, f. OC-O stretching and g. symmetric COC stretching. The combined surface of peaks a and $b$ and the combined surface of peaks $f$ and $g$ were used to calculate the $\mathrm{CH} 2 / \mathrm{C}-\mathrm{O}$ ratio which could give an indication to the degree of saponification on the PCL microsphere surface.

B. Semiquantitative analysis of the changes in $\mathrm{CH} 2$ and $\mathrm{C}-\mathrm{O}$ FTIR peak integral ratio because of exposure to $0.1 \mathrm{M} \mathrm{NaOH}$ for varying durations $(n=3)$. $(*=p<0.05)$

C. PCL microspheres prepared by sonication induced emulsification show no apparent sign of agglomeration or shape-loss after 30 minutes of exposure to $0.1 \mathrm{M}$ $\mathrm{NaOH}$. $\mathrm{NaOH}$ exposure does not result in observable changes to the surface morphology of the microspheres.

D. Molecular structure of ALN and its fluorescent model EDANS. The primary amine on the short carbohydrate tether in both molecules result in similar EDC/NHS based conjugation to the surface of carboxylated PCL microspheres.

E. Conjugation procedure of EDANS to a PCL surface enriched with carboxylic acid groups by $\mathrm{NaOH}$ induced saponification. After EDC/NHS activation of surface carboxylic acids, the fluorescent ALN model molecule EDANS was conjugated to the PCL microsphere surface.

F. Fluorescent intensity of the surface conjugated EDANS molecules on PCL microspheres after varying $\mathrm{NaOH}$ saponification times and EDC/NHS activation of surface carboxylic acids. 
Table 3.2 - Mw, Mn and PDI parameters of PCL microspheres and saponificated PCL microspheres assessed by GPC analysis

\begin{tabular}{cccc}
\hline $\begin{array}{c}\text { Microsphere } \\
\text { group }\end{array}$ & Mw & Mn & $\begin{array}{c}\text { PDI } \\
(\mathbf{M w} / \mathbf{M n})\end{array}$ \\
\hline PCL & $84300 \mathrm{~g} \cdot \mathrm{mol}^{-1}$ & $47200 \mathrm{~g} \cdot \mathrm{mol}^{-1}$ & 1.78 \\
Saponificated PCL & $82300 \mathrm{~g} \cdot \mathrm{mol}^{-1}$ & $45000 \mathrm{~g} \cdot \mathrm{mol}^{-1}$ & 1.83 \\
\hline
\end{tabular}

Surface morphology of treated microspheres remains smooth without the presence of pores or defects (Figure 3.4C). In order to quantify the amount of ALN which can be grafted onto the carboxyl enriched PCL surface, a fluorescent probe (EDANS) was selected, which exhibits similarities in terms of physio-chemical properties and molecular weight (comparison with ALN shown in Figure 3.4D). Figure 3.4E shows a schematic of the reaction that followed the microsphere saponification. The combinational study with varying $\mathrm{NaOH}$ treatment durations and EDC/NHS carboxyl activation times (Figure 3.4F) shows that when both steps are performed for at least 30 minutes there is no additional improvement in EDANS functionalization when prolonging treatment duration. Without saponification, the EDANS fluorescence intensity decreased three-fold and without EDC/NHS conjugation chemistry, no covalent binding of EDANS occurred.

\section{PCL-ALN microspheres exhibit high HAP binding properties}

A scaffold made from porous bone like material, previously described by our group [23], was used to assess the complexation of PCL-ALN microspheres with HAP (Figure 3.5A). A 15-fold increase in fluorescence could be measured when PCL-ALN microspheres (loaded with pyrene) were 
compared with non-functionalized microspheres PCL loaded with pyrene after incubation with the scaffold (Figure 3.5B).

A.

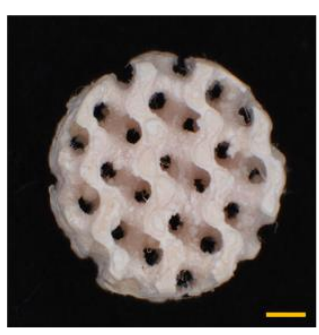

B.

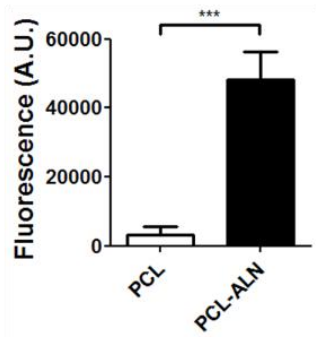

C.

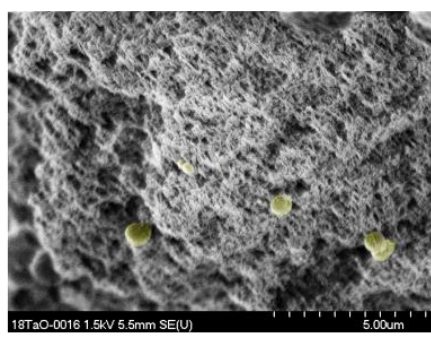

Figure 3.5 - Interaction of ALN functionalized microspheres with HAP-containing substrates.

A. Macroporous methacrylated poly(trimethylene carbonate) scaffold with $40 \mathrm{wt} \%$ nano-HAP crystals. The gyroid pore structures, established by stereolithography, could be interpreted as a mimicking structure for trabecular bone. The scalebar represents a length of $1 \mathrm{~mm}$. Picture republished with permission of [25].

B. PCL-ALN microspheres loaded with fluorescent pyrene bound 15 times more effectively to PTMC/40\%HAP scaffolds compared to PCL microspheres. (unpaired t-test, $\alpha=0.05 \mathrm{p}=0.0008$ ).

C. SEM depicting PCL-ALN microspheres bound to a HAP containing substrate. Microparticles were highlighted by artificial yellow coloring using Photoshop CS5.

The increased fluorescence signals from the bound PCL-ALN microspheres demonstrates that the surface ALN groups are actively involved in chelation to the bone like material. The presence of microspheres adsorbed onto the surface of the porous HAP scaffold could additionally be observed using SEM (Figure 3.5C), with chelated PCL-ALN microspheres indicated by the yellow color. The SEM analysis, together with the increased fluorescence, support the claim that the designed DDS is actively targeting bone mineral.

PCL-ALN microspheres do not hinder bone resorption by osteoclasts

Osteoclast specific TRAP staining (Figure 3.6A) showed that in normal osteoclast culture conditions, large and multinucleated cells are formed and 
are intensely stained. In the presence of $10 \mu \mathrm{M} A L N$, the morphology of the osteoclasts became smaller and rounded up, indicating inhibitory and potentially cytotoxic effects of ALN. In the presence of PCL-ALN microspheres, the morphology of osteoclasts remains comparable to untreated cells, which are multinucleated and TRAP positive. A trend was observable for PCL-ALN treated cells to have fewer nuclei, or to be smaller in diameter (Figure 3.6A).

The inhibitory properties of the PCL, PCL-ALN and ALN on the ability of osteoclasts to resorb HAP was showed by a von Kossa staining (example shown in Figure 3.6B). The data in Figure 3.6C reveals that the untreated osteoclasts (M-CSF) show very high HAP resorption $(89.5 \%$ of total surface). The presence of $1.7 \mathrm{mg}$ of PCL microspheres reduced the resorptive potential of osteoclasts by approximately $25 \%$. This may be due to the precursor cells not forming osteoclasts after phagocytizing the PCL microspheres and affecting their differentiation. When the PCL microspheres were functionalized with ALN, the HAP resorption did not differ significantly from the non-functionalized group. In contrast, $10 \mu \mathrm{M}$ ALN further reduced the HAP resorption by approximately 35\%. Interestingly, ALN functionalization of the microspheres did not inhibit resorption greater than PCL alone. 

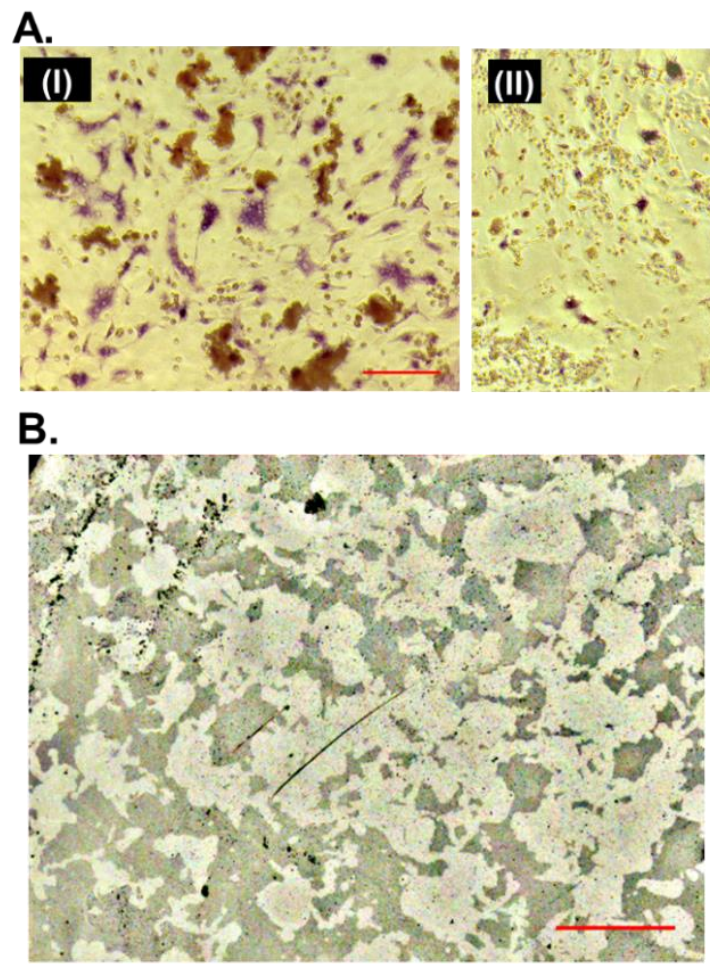
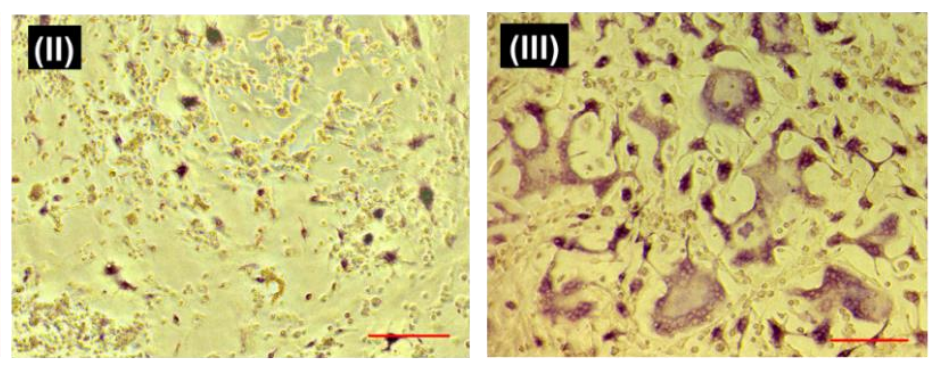

C.

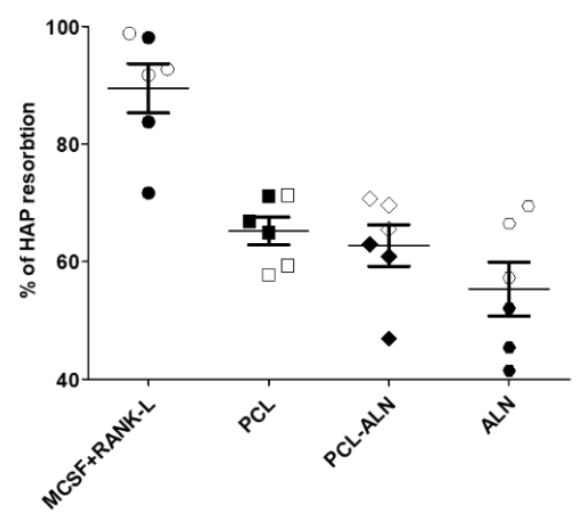

Figure 3.6 - Influence of ALN functionalized PCL microspheres on osteoclastic HAP resorption.

A. Morphology of murine osteoclasts cultured in presence of $1.7 \mathrm{mg}$ PCL-ALN microspheres (I), a $10 \mu \mathrm{M}$ ALN solution (II) and a control culture without the presence of solubilized ALN or PCL-ALN microspheres (III). Scalebar equals 50 $\mu \mathrm{m}$.

B. Representative image of a Von Kossa stained Osteoassay ${ }^{\circledR}$ well plate substrate exposed to $1.7 \mathrm{mg}$ PCL-ALN microspheres. The osteoclasts grew for 72 hours on the mineral substrate before they were removed. The dark areas are stained remnants of the HAP substrate while the lighter areas show the resorption by the osteoclasts. Scalebar equals $200 \mu \mathrm{m}$.

C. Percentage of HAP substrate surface resorbed by osteoclasts in the presence of PCL microparticles, PCL-ALN microparticles or $10 \mu \mathrm{M}$ ALN solution. MCSF+RANK-L group represents a control group without any microparticles or ALN addition. The full and empty symbols represent two murine macrophage donors. 


\section{GM-AOT loaded PCL microspheres exhibit prolonged antimicrobial properties}

The MIC and MIB concentrations of GM-AOT, its parent drug GM and the GM-AOT loaded PCL microspheres can be found in Table 3.3.

Table 3.3 - Susceptibility of $S$. aureus for GM, GM-AOT and PCL/GM-AOT microspheres

\begin{tabular}{ccc}
\hline Group & MIC $(\boldsymbol{\mu g} / \mathbf{m L})$ & MBC $(\boldsymbol{\mu g} / \mathbf{m L})$ \\
\hline GM & 1.95 & 3.91 \\
GM-AOT & 0.98 & 0.98 \\
PCL/GM-AOT & 9.76 & 19.53 \\
microspheres & & \\
\hline
\end{tabular}

The evolution of the agar plate zone of inhibition conducted on $S$. aureus demonstrated that, when administrated as single dose, GM-AOT in DMSO solution has a limited duration of antimicrobial effect (Figure 3.7A). Prolonged antimicrobial effects were observed for the tested reference materials that are currently employed in clinics. PMMA bone cement (Copal $\mathbb{R})$ loaded with a combination of GM and Vancomycin was responsible for an intermediate $\mathrm{ZOI}$ on the $S$. aureus infected agar plates but had a consisted bactericidal effect throughout the 7-day experiment. The GM loaded collagen fleece (Garamycin ${ }^{\circledR}$ ) released its drug content in a singular burst resulting in very large inhibitory zones in only the first 24 hours, followed by a rapid decline of ZOI. This observation supports previous publications, describing that the majority of gentamicin was released from collagen sponge materials after 1.5 hours [26]. PCL microspheres loaded with GM-AOT had an antimicrobial effect up to 5 days (Figure 3.7B). 
A.

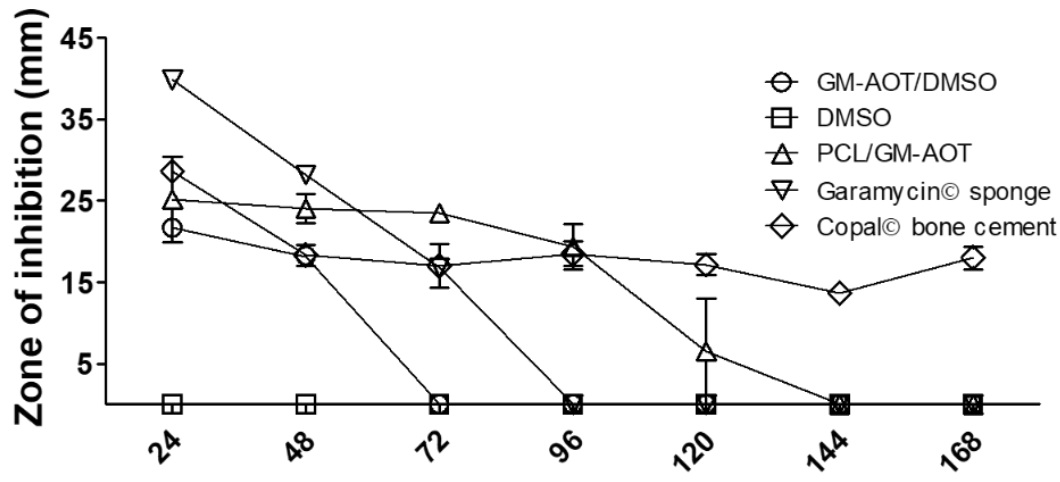

B.

Time (h)

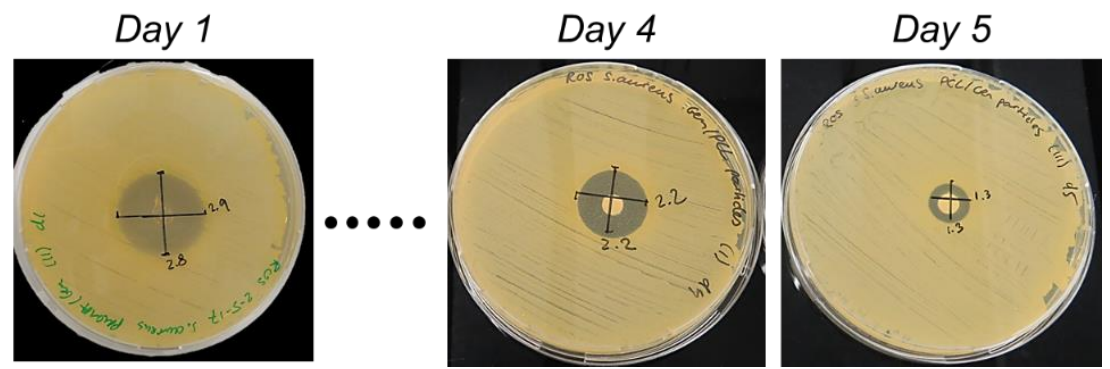

Figure 3.7 - Antimicrobial effect of GM-AOT loaded microparticles on Grampositive $S$. aureus.

A. Comparison of the GM-AOT loaded PCL microspheres to a GM-AOT solution. The solvent, DMSO, had no measurable inhibitive effect on S. aureus colonization. Garamycin ${ }^{\circledR}$ sponge and Copal Bone cement are two established antibiotic delivery materials

B. Photographs of the zone of inhibition of S. aureus colonization due to the release of GM-AOT from PCL microspheres. Wafer containing $1 \mathrm{mg}$ of PCL microspheres loaded with GM-AOT was transferred daily to an agar plate with freshly plated $S$. aureus.

\section{Discussion}

The current systemic antibiotic therapies for prevention or treatment of bone infection have reduced efficacy due to the combination of low availability at the bony site and the antibiotic's short biological half-lives. Those short-comings make utilization of antibiotic loaded biomaterials a near mandatory step in order to help OM patients to successfully recover 
[27]. However, drug release mismatch with desired release patterns, along with lack of biodegradable properties are pressing issues characterizing the available DDS. Ideally, the DDS needs to release its complete antibiotic load within 2 to 3 weeks after implantation to eradicate the bone infection and to prevent sub-inhibitory antibiotic concentrations leaching from the carrier material in the subsequent periods. Prolonged exposure to antibiotic concentrations below the MIC can promote specific antibiotic resistances [28]. The presented DDS aims to overcome these challenges by enhancing drug diffusion kinetics through the utilization of micro sized particles exhibiting large surface area and composed of biodegradable polymers.

In this study, we showed that by varying the nature of the polymer matrix and the emulsion method, we can tailor hydrophobic GM-AOT release from the fabricated polyester microspheres. PDLLA and PCL materials released the loaded drugs with different release rates. It is hypothesized that drug content in PCL microspheres is more located at the surface of the particle due to its higher crystallinity and is released faster in a diffusion driven release mechanism. In contrast, once encapsulated in more amorphous PDLLA microspheres GM-AOT is better retained in the matrix, resulting in a lower cumulative release in 14 days. This effect, already reported by other groups $[29,30]$ can be explained by differences in microsphere size, polymer crystallinity [31] and molecular weight [32] of the polymers used. It is considered that polymeric matrices with high crystallinity can lead to an increased release of the encapsulated drugs due to the polymer's conformation on the atomic level that expedite the outward diffusion of the drugs [33]. Generally, crystalline materials have poorer drug encapsulation than their amorphous counterparts [29,34], which is supported by the data in Figure 3.2. 
Drug delivery of other hydrophobic drugs like doxorubicin has been investigated and the relation between DDS parameters and drug release have been reported. Amjadi et al. prepared doxorubicin loaded PLGA particles in which the lactide content of $50 \%$ and $75 \%$ was used [32]. Increasing lactide content of the PLGA resulted in lower doxorubicin EE\% and lower polydispersity while crystallinity was increased. The release profile of the doxorubicin was faster for the more crystalline PLGA with $75 \%$ lactide content compared to the more amorphous PLGA with 50\% lactide content. Similar finding was presented by Guillaume et al. [29] comparing the release kinetic and remaining drug retention of another antibiotic (ofloxacin) from amorphous PDLLA versus crystalline PCL, corroborating our results presented here.

The size distribution of the microspheres affects the drug release profile as well. For example, PDLLA/GM-AOT/HSM batches with the highest polydispersity and skewed microsphere size distribution showed the slowest release of GM-AOT. Spiridonova et al. performed extensive modeling on the drug release properties of PLGA delivery vesicles with many geometries, including spherical structures [35]. Findings included that polydisperse spherical systems released their drug slower compared to a more monodisperse system with the same average microsphere diameter. It was reasoned that out-ward diffusion of drug encapsulated in larger microspheres is limited to drug at the periphery of the polymer matrix. This is presumably the reason for the large portion of GM-AOT that remains unreleased in PDLLA/GM-AOT/HSM microspheres. Spiridonova et al. also stated that polydisperse microspheres did significantly not affect drug diffusion in the first hours of release [35], which matches the results presented in this work. 
The sustained release of GM-AOT form both PCL and PDLLA matrices fitted to the Korsmeyer-Peppas model for sustained drug release from polymeric matrices. For spherical systems, values of $n<0.43$ represent drug release via Fickian diffusion. All four $n$ values of the release profiles are below 0.43 , suggesting a release fitting the Fickian diffusion. Low $n$ values could be expected due to the high molecular weight of the PCL and PDLLA polymers and the slow in vitro degradation of these polyesters. Ribeiro et al. implemented the Korsmeyer-Peppas model to fit their curcumin release from polyester nanoparticles [36]. With reported $n$ values between 0.31 and 0.40 for different PLGA and PCL compositions, their results are comparable to the data presented in this work.

Average molecular weight of the polymers was only minimally affected by exposure to $\mathrm{NaOH}$, suggesting that saponification was primarily occurring at the microspheres surface. This is in accordance with previous reports on saponification of PCL fibers [37]. EDC/NHS conjugation of ALN to polyester surfaces was optimized in this study. Even though the chemistry is known for decades as an effective conjugation method, protocols vary depending on the nature of the conjugated molecule. While often applied for conjugation of solubilized components, functionalization of surfaces is less frequently reported. We observed that an increase in saponification time yielded a (slightly) higher carboxylic acid presence on the polyester surface, but it did not influence the binding efficacy of EDANS (fluorophore model for ALN). This observation could be caused by the saturation of the microsphere surface with EDANS due to repulsive forces of the sulfonic acid groups and their negatively charged sulfonate conjugated base. For the targeted application requiring the utilization of the bone seeker ALN, 
grafting large amounts of ALN onto the microsphere surface is not essential for an efficient functionality. On the contrary, spherical drug carriers with lower amounts of surface bisphosphonate groups were shown to be more stable in biological environments and to perform similarly in regard to bone binding efficacy as their counterparts with a denser presence of active surface moieties [38].

The bone binding capacity of the microspheres give potential for targeted drug delivery to bone. However, possible side effects of ALN on bone homeostasis should be investigated. All BPs are known to inhibit osteoclast ability to resorb bone, which makes them excellent drugs for treating osteoporosis or Paget's disease [39]. Because physiological bone homeostasis is essential during the healing process of OM, it should not be impeded. Hence, using BPs as targeting ligands on DDS needs to be cautiously considered. This study describes the effect of PCL-ALN microspheres and ALN solutions on osteoclast formation and their ability to resorb HAP. We show that the presence of a high concentration of PCL microspheres already influences the ability of osteoclasts to resorb bone in a $2 \mathrm{D}$ in vitro model. After surface functionalization with ALN, no significant differences were observed in comparison with the PCL group. These results were coherent with findings from Cenni et al. who assessed ALN functionalized PLGA microspheres [40].

Osteoclasts inherently have broad size distributions, with larger cells being more efficient in bone resorption. When osteoclast size was assessed using TRAP staining, it was observed that the presence of ALN resulted in smaller osteoclasts. Though still observable, this effect was less evident for osteoclasts cultured with PCL-ALN microspheres. These results confirm 
that when ALN is present on the surface of a polymer construct, it has less detrimental effects on osteoclast growth than when ALN is in solution. This finding can be explained by the fact that the primary amine group of ALN is known to be a promotor of osteoclast inhibition $[41,42]$. Because the primary amine of ALN is required for the conjugation chemistry to the PCL microspheres, its effect on inhibition of HAP resorption is diminished. Another reason for the similar effects of PCL and PCL-ALN might be the limited amount of ALN that is in direct contact with the HAP substrate and the underlying osteoclasts. A large portion of ALN grafted onto the particle surface is not in direct contact with the osteoclasts on the HAP substrate, hereby potentially reducing the amount of ALN acting on the osteoclasts.

Due to the sustained release of antibiotic, the MIC and MBC of the developed PCL microspheres with GM-AOT load is approximately 10 times higher than the MIC and MBC for solubilized GM-AOT. Interestingly, GMAOT showed a lower MIC and MBC as its parent drug GM. This phenomenon was also observed by Ter Boo et al. and was accredited to the destabilizing properties of the AOT ion on the bacterial membrane [9].

Different bacterial strains have been identified to reside at the sites of OM, with the major bacterial strain being $S$. aureus [42]. In terms of antimicrobial activity against $S$. aureus, the PCL microspheres loaded with GM-AOT was as efficient as the clinical reference materials, e.g. Garamycin ${ }^{\circledR}$ collagen sponge and Copal ${ }^{\circledR}$ bone cement. The microspheres inhibited S. aureus for a longer duration than the collagen sponge and had the biodegradable properties that the PMMA bone cement is lacking. Although Copal® bone cement had a longer antimicrobial effect compared to the designed microspheres, GM-AOT loaded PCL microspheres had a larger zone of 
inhibition for the initial 4 days of the experiment. The great advantage of the developed PCL microspheres does not only lie in its specific affinity towards bone tissue, but also in the fact that its biodegradable properties will make future retrieval surgeries unnecessary, which is beneficial to the patient's recovery process.

\section{Conclusion}

Further developments in targeted antibiotic delivery to bone are crucial due to the currently inadequate success rate of OM treatment. This work describes the successful development of antibiotic loaded PCL and PDLLA microspheres. Sustained in vitro antibiotic release allowed for an antimicrobial efficacy against the major $\mathrm{OM}$ microbial, $S$. aureus, for up to 5 days. Saponification of the microspheres' surface allowed efficient covalent conjugation of ALN which resulted in 15 times increase in microsphere binding to bone mimicking materials. This shows that our designed DDS can actively bind to HAP and deliver antibiotics to bone mimicking sites. Due to the versatile nature of the polyester microspheres, other hydrophobic drugs can potentially be encapsulated to optimize the treatment of other bone related diseases such as osteoporosis or even osteosarcoma.

\section{Funding/Support statement}

This work was supported by the AOTrauma Clinical Priority Program Bone Infection. 


\section{Acknowledgements}

Alexandra Wallimann and the Swiss Institute of Allergy and Asthma Research is kindly thanked for the donation of murine bone marrow derived macrophages.

\section{References}

1 Patzakis, M. \& G Zalavras, C. Chronic posttraumatic osteomyelitis and infected nonunion of the tibia: current management concepts. Journal of the American Academy of Orthopaedic Surgeons, Vol. 13 (2005).

2 Darley, E. S. R. \& MacGowan, A. P. Antibiotic treatment of Gram-positive bone and joint infections. Journal of Antimicrobial Chemotherapy 53, 928-935, doi:10.1093/jac/dkh191 (2004).

3 Isefuku, S., Joyner, C. J. \& Simpson, A. H. R. W. Gentamicin may have an adverse effect on osteogenesis. Journal of orthopaedic trauma 17, 212-216, doi:10.1097/00005131-200303000-00010 (2003).

4 Hatzenbuehler, J. \& Pulling, T. J. Diagnosis and management of osteomyelitis. American family physician 84, 1027-1033 (2011).

5 Schmidmaier, G., Gahukamble, A. D., Moriarty, T. F. \& Richards, R. G. in Biomaterials Associated Infection: Immunological Aspects and Antimicrobial Strategies (eds T. Fintan Moriarty, Sebastian A. J. Zaat, \& Henk J. Busscher) 435-453 (Springer New York, 2013).

6 Geurts, J. A. P. \& Walenkamp, G. H. I. M. in Management of Periprosthetic Joint Infections (PJIs) 219-230 (Woodhead Publishing, 2017).

7 Ze Liu, H., Qi, M., Guo, B. \& Hua Liu, H. Effects of Hydrophilicity/Hydrophobicity of a Drug on its Release from PLGA Films. Material Science Forum Vol. 675-677 (2011).

8 Schnieders, J., Gbureck, U., Thull, R. \& Kissel, T. Controlled release of gentamicin from calcium phosphate-poly(lactic acid-co-glycolic acid) composite bone cement. Biomaterials 27, 4239-4249, doi:https://doi.org/10.1016/j.biomaterials.2006.03.032 (2006).

9 Boo, G.-J., W Grijpma, D., Richards, R., Moriarty, T. \& Eglin, D. Preparation of gentamicin dioctyl sulfosuccinate loaded poly(trimethylene carbonate) matrices intended for the treatment of orthopaedic infections. Vol. 60 (2015).

10 Imbuluzqueta, E. et al. Novel bioactive hydrophobic gentamicin carriers for the treatment of intracellular bacterial infections. Acta Biomater 7, 1599-1608, doi:10.1016/j.actbio.2010.11.031 (2011).

11 Sánchez, E., Baro, M., Soriano, I., Perera, A. \& Évora, C. In vivo-in vitro study of biodegradable and osteointegrable gentamicin bone implants. European Journal of Pharmacentics and Biopharmaceutics 52, 151-158, doi:https://doi.org/10.1016/S09396411(01)00169-2 (2001).

12 Ungaro, F. et al. Dry powders based on PLGA nanoparticles for pulmonary delivery of antibiotics: Modulation of encapsulation efficiency, release rate and 
lung deposition pattern by hydrophilic polymers. Journal of Controlled Release 157, 149-159, doi:https://doi.org/10.1016/i.jconrel.2011.08.010 (2012).

Chang, H. I., Perrie, Y. \& Coombes, A. G. A. Delivery of the antibiotic gentamicin sulphate from precipitation cast matrices of polycaprolactone. Journal of Controlled Release 110, 414-421, doi:https://doi.org/10.1016/j.jconrel.2005.10.028 (2006).

Cha, Y. \& Pitt, C. G. The biodegradability of polyester blends. Biomaterials 11, 108112, doi:https://doi.org/10.1016/0142-9612(90)90124-9 (1990).

Rotman, S. G. et al. Drug delivery systems functionalized with bone mineral seeking agents for bone targeted therapeutics. J Control Release 269, 88-99, doi:10.1016/j.jconrel.2017.11.009 (2018).

Yewle, J. N. BIFUNCTIONAL BISPHOSPHONATES FOR DELIVERING BIOMOLECULES TO BONE PhD thesis, (2012).

Choi, S. W. \& Kim, J. H. Design of surface-modified poly(D,L-lactide-coglycolide) nanoparticles for targeted drug delivery to bone. J Control Release 122, 2430, doi:10.1016/j.jconrel.2007.06.003 (2007).

8 de Miguel, L., Noiray, M., Surpateanu, G., Iorga, B. I. \& Ponchel, G. Poly( $\gamma$-benzyll-glutamate)-PEG-alendronate multivalent nanoparticles for bone targeting. International Journal of Pharmaceutics 460, 73-82, doi:http://dx.doi.org/10.1016/j.ijpharm.2013.10.048 (2014).

Thamake, S. I., Raut, S. L., Gryczynski, Z., Ranjan, A. P. \& Vishwanatha, J. K. Alendronate coated poly-lactic-co-glycolic acid (PLGA) nanoparticles for active targeting of metastatic breast cancer. Biomaterials 33, 7164-7173, doi:http://dx.doi.org/10.1016/j.biomaterials.2012.06.026 (2012).

20 Pranatharthiharan, S. et al. Asialoglycoprotein receptor targeted delivery of doxorubicin nanoparticles for hepatocellular carcinoma. Drug delivery 24, 20-29, doi:10.1080/10717544.2016.1225856 (2017).

21 Moshiri, A., Sharifi, A. M. \& Oryan, A. Role of Simvastatin on fracture healing and osteoporosis: a systematic review on in vivo investigations. Clinical and Experimental Pharmacology and Physiology 43, 659-684, doi:10.1111/1440-1681.12577 (2016).

Segal, E. et al. Enhanced anti-tumor activity and safety profile of targeted nanoscaled HPMA copolymer-alendronate-TNP-470 conjugate in the treatment of bone malignances. Vol. 32 (2011).

23 Guillaume, O. et al. Surface-enrichment with hydroxyapatite nanoparticles in stereolithography-fabricated composite polymer scaffolds promotes bone repair. Acta Biomater 54, 386-398, doi:10.1016/j.actbio.2017.03.006 (2017).

24 Sampath, S. S. \& Robinson, D. H. Comparison of new and existing spectrophotometric methods for the analysis of tobramycin and other aminoglycosides. Journal of pharmaceutical sciences 79, 428-431 (1990).

25 Guillaume, O. et al. Poly(trimethylene carbonate) and nano-hydroxyapatite porous scaffolds manufactured by stereolithography. Polymers for Advanced Technologies, Vol. 28 (2016).

26 Sorensen, T. S., Sorensen, A. I. \& Merser, S. Rapid release of gentamicin from collagen sponge. In vitro comparison with plastic beads. Acta orthopaedica Scandinavica 61, 353-356, doi:10.3109/17453679008993535 (1990).

27 Neut, D. et al. A biodegradable antibiotic delivery system based on poly(trimethylene carbonate) for the treatment of osteomyelitis. Acta Orthop 80, 514519, doi:10.3109/17453670903350040 (2009). 

(2011).

29 Guillaume, O. et al. New antibiotic-eluting mesh used for soft tissue reinforcement. Acta Biomater 7, 3390-3397, doi:10.1016/j.actbio.2011.05.009 (2011).

Liggins, R. T. \& Burt, H. M. Paclitaxel loaded poly(l-lactic acid) (PLLA) microspheres: II. The effect of processing parameters on microsphere morphology and drug release kinetics. International Journal of Pharmaceutics 281, 103-106, doi:https://doi.org/10.1016/i.ijpharm.2004.05.027 (2004).

31 Yan, J. et al. Fine Tuning Micellar Core-Forming Block of Poly(ethylene glycol)block-poly( $\varepsilon$-caprolactone) Amphiphilic Copolymers Based on Chemical Modification for the Solubilization and Delivery of Doxorubicin. Biomacromolecules 12, 2562-2572, doi:10.1021/bm200375x (2011).

Sunoqrot, S., Alsadi, A., Tarawneh, O. \& Hamed, R. Polymer type and molecular weight dictate the encapsulation efficiency and release of Quercetin from polymeric micelles. Colloid and Polymer Science 295, 2051-2059, doi:10.1007/s00396017-4183-9 (2017).

33 Sinha, V. R., Bansal, K., Kaushik, R., Kumria, R. \& Trehan, A. Poly-€-caprolactone microspheres and nanospheres: an overview. International Journal of Pharmaceutics 278, 1-23, doi:https://doi.org/10.1016/i.ijpharm.2004.01.044 (2004).

Korzhikov, V., Averianov, I., Litvinchuk, E. \& Tennikova, T. B. Polyester-based microparticles of different hydrophobicity: the patterns of lipophilic drug entrapment and release. Journal of Microencapsulation 33, 199-208, doi:10.3109/02652048.2016.1144818 (2016).

Spiridonova, T. I., Tverdokhlebov, S. I. \& Anissimov, Y. G. Investigation of the Size Distribution for Diffusion-Controlled Drug Release From Drug Delivery Systems of Various Geometries. Journal of pharmacentical sciences, doi:https://doi.org/10.1016/j.xphs.2019.03.036 (2019).

Ribeiro, C. A. S., de Castro, C. E., Albuquerque, L. J. C., Batista, C. C. S. \& Giacomelli, F. C. Biodegradable nanoparticles as nanomedicines: are drug-loading content and release mechanism dictated by particle density? Colloid and Polymer Science 295, 1271-1280, doi:10.1007/s00396-016-4007-3 (2017).

37 Bosworth, L. A., Hu, W., Shi, Y. \& Cartmell, S. H. Enhancing Biocompatibility without Compromising Material Properties: An Optimised $\mathrm{NaOH}$ Treatment for Electrospun Polycaprolactone Fibres. Journal of Nanomaterials 2019, 11, doi:10.1155/2019/4605092 (2019).

38 Yin, Q. et al. Pamidronate functionalized nanoconjugates for targeted therapy of focal skeletal malignant osteolysis. Proceedings of the National Academy of Sciences, doi:10.1073/pnas.1603316113 (2016).

39 Fleisch, H. Bisphosphonates in bone disease: From laboatory to the patient. 2nd edn, (The Parthenon publishing group Ltd., 1995).

40 Cenni, E. et al. The effect of poly(d,l-lactide-co-glycolide)-alendronate conjugate nanoparticles on human osteoclast precursors. J Biomater Sci Polym Ed 23, 1285 1300, doi:10.1163/092050611X580373 (2012).

41 Drake, M. T., Clarke, B. L. \& Khosla, S. Bisphosphonates: Mechanism of Action and Role in Clinical Practice. Mayo Clinic proceedings. Mayo Clinic 83, 1032-1045 (2008).

42 Dunford, J. E. et al. Structure-activity relationships for inhibition of farnesyl diphosphate synthase in vitro and inhibition of bone resorption in vivo by 
nitrogen-containing bisphosphonates. The Journal of pharmacology and experimental therapeutics 296, 235-242 (2001). 


\section{Chapter 3 - Appendix}

\section{Evaluation of bone targeting and antimicrobial properties of functionalized PCL microspheres in rat femoral defects}

S.G. Rotman ${ }^{1,2}$, D.W. Grijpma ${ }^{2}$, T.T. Tang ${ }^{3}$, D. Eglin ${ }^{1,2}$ and O. Guillaume ${ }^{1}$

${ }^{1}$ AO Research Institute Davos, Davos Platz, Switzerland

${ }^{2}$ Department of Biomaterials Science and Technology, Faculty of Science and Technology, University of Twente, Enschede, The Netherlands

${ }^{3}$ Shanghai Key Laboratory of Orthopedic Implants, Department of Orthopedic Surgery, Shanghai Ninth People's Hospital, Shanghai Jiao Tong University School of Medicine, Shanghai, China

Unpublished dataset 


\section{Introduction}

The assessment of in vivo performance of new biomaterials forms an important step that bridges the gap between promising in vitro results and the start of phase I clinical trials. With a wide variety of available animal models with different levels of complexity, in vivo characterization of a new biomaterial likely spans over several animal experiments, ranging from initial basic toxicity investigations to more complex animal models that are more clinically relevant and assessing efficacy. In vivo models often involve small animals such as mice and rats, while large animal models (e.g. sheep or pigs) are used scarcely when the human anatomy and pathology needs to be mimicked more closely. This also applies to investigation of antimicrobial biomaterials used to assess new treatments or prevention of musculoskeletal infections in which promising results in a small animal model justify further testing in complex and expensive large animal models $[1,2]$. Throughout the studies in this thesis, several early-stage in vivo assessments took place in which newly fabricated biomaterials were tested in murine and rat infection models.

In this Appendix, unpublished in vivo data on bone binding properties and antimicrobial potential of antibiotic loaded microspheres introduced in Chapter 3. A direct comparison in the bone binding potential of PCL microspheres functionalized with Alendronate (ALN) or poly(aspartic acid) (ASP) was made. Furthermore, the antimicrobial properties of GM-AOT loaded PCL microspheres were shown in a rat femoral head infection model. 


\section{Materials and methods}

\section{Materials}

PCL $\left(\mathrm{M}_{\mathrm{w}}=80,000 \mathrm{~g} \cdot \mathrm{mol}^{-1}\right.$, polydispersity index $\left.<2\right)$, IR-780 iodide dye, ALN, N-hydroxysuccinimide (NHS), gentamicin sulfate salt, phosphate buffered saline (PBS) and dioctyl sodium sulfosuccinate sodium salt (AOT) were purchased from Sigma Aldrich (Steinheim, Germany). Poly(vinyl alcohol) $\left(\mathrm{PVA}, \mathrm{M}_{\mathrm{w}}=31.000 \mathrm{~g} \cdot \mathrm{mol}^{-1}\right)$, sodium hydroxide $(\mathrm{NaOH})$, dichloromethane $\left(\mathrm{CH}_{2} \mathrm{Cl}_{2}\right)$, dimethyl sulfoxide (DMSO) and 2-propanol was bought from Carl Roth (Karlsruhe, Germany). (D-ASP)6 oligomers were synthesized on demand by GenScript (Leiden, the Netherlands). Ethyl-3-(3dimethylaminopropyl)carbodiimide (EDC) was purchased from Fluka (Buchs, Switzerland). Tryptone soy agar (TSA) was purchased from Oxoid AG (Basel, Switzerland).

\section{Microsphere fabrication}

Microspheres were fabricated as described in Chapter 3 and its original publication [3]. Briefly, a 10\% PCL solution in $\mathrm{CH}_{2} \mathrm{Cl}_{2}$ was prepared. As encapsulated compounds, 25\% w/w GM-AOT or IR-780 iodide (control IR fluorescent dye) relative to the polymer weight, was added to the solution. Solubilized PVA $(100 \mathrm{~mL}, 1 \% \mathrm{w} / \mathrm{v})$ acted as the aqueous phase during the emulsion process. Emulsions were prepared by probe sonication (Bandelin Sonopuls GM70, 3 bursts of 10 seconds, 700W). The organic solvent was evaporated over 4 hours at room temperature under moderate stirring. The microspheres were saponificated for 60 minutes in $0.1 \mathrm{M} \mathrm{NaOH}$, and then dispersed in $10 \mathrm{~mL}$ 2-(N-morpholino)ethanesulfonic acid (MES)-buffer (pH $=5.5$ ) containing EDC (50 mg, $0.26 \mathrm{mmol})$ and NHS (50 mg, $0.43 \mathrm{mmol}$ ). 
Alendronate (ALN) or Aspartic acid oligomers (ASP) were then added to the microsphere dispersion and the reaction occurred under ambient conditions for 2 hours. The microsphere dispersions were flash-frozen in liquid $\mathrm{N}_{2}$ and lyophilized.

\section{Rat femoral head defect model and treatment regimes}

Wild type Sprague Dawley rats (male) were anesthetized by $1 \mathrm{~mL}$ abdominal injections of $9.1 \mathrm{mg} / \mathrm{mL}$ chloral hydrate solution. The right upper-hind leg area of the rats was shaved and cleaned with $70 \%$ ethanol. After making a small incision and gently maneuvering the leg, the femur was exposed with minimal muscle damage. A 2-mm circular defect, approximately $5 \mathrm{~mm}$ deep, was drilled at the proximal part of the femur, the femoral head. Next, $10 \mu \mathrm{L}$ of Staphylococcus aureus Xen-29 bacterial dispersion $\left(10^{8} \mathrm{CFU} / \mathrm{mL}\right)$ was pipetted into the bone defects and covered with hemostatic bone wax to prevent rapid wash-out of the bacteria due to bleeding. The incisions were sutured closed and the infections could progress for 7 days. At day 7 , the animals were treated with GM-AOT loaded PCL microsphere dispersions in PBS. $20 \mu \mathrm{L}$ of dispersions $(50 \mathrm{mg} / \mathrm{mL}$ ) were pipetted into the defect and the defects were again covered with hemostatic bone wax to prevent rapid washout of the microspheres. The animals did not receive systemic antibiotic throughout the experiment.

On day 14, the rats were euthanized by cervical dislocation. The femurs were harvested, and the bioluminescent $S$. aureus Xen-29 bacteria were imaged with a Spectrum In Vivo Imaging System (IVIS, Perkin Elmer). After imaging, the proximal parts of the femurs were homogenized, and bacteria were quantified by after plating on TSA plates. 
The experiments were approved by the Animal Ethics Committee of Shanghai Jiaotong University School of Medicine.

\section{Results and Discussion}

\section{Microsphere retention of IR-780 iodide dye}

IR-780 iodide dye is encapsulated in the microspheres for utilization in localization and quantification via in situ fluorescence imaging. Therefore, to evaluate the validity of future in vivo imaging, it is important to initially assess potential leaching of IR-780 iodide from the microspheres. Figure 3A.1 shows the near infra-red fluorescent imaging (A.) and quantification of fluorescent signals (B.) of the supernatants that contained IR-780 iodide loaded PCL, PCL-ALN and PCL-ASP microspheres. After 7 days of incubation in water, the near infra-red fluorescent signals of IR-780 iodide loaded microspheres (Pellet) is also measured. Results in Figure 3A.1 show that the dye remains embedded within the microspheres with minimal leeching of dye observed in the supernatants. This result validates the us of the IR-780 for in vivo imaging by observing the near infra-red fluorescent signals of the IR-780 iodide dye loaded microspheres.

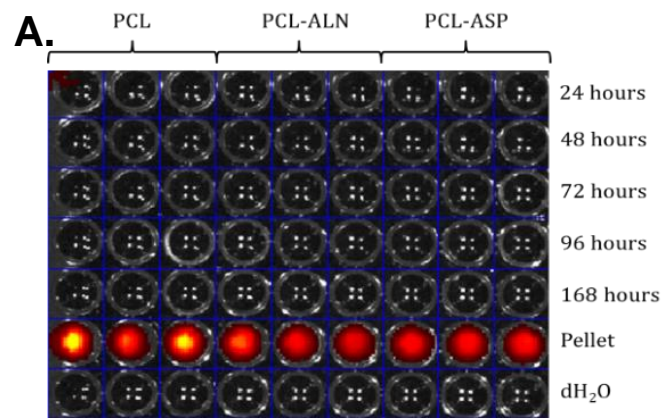

\section{B.}

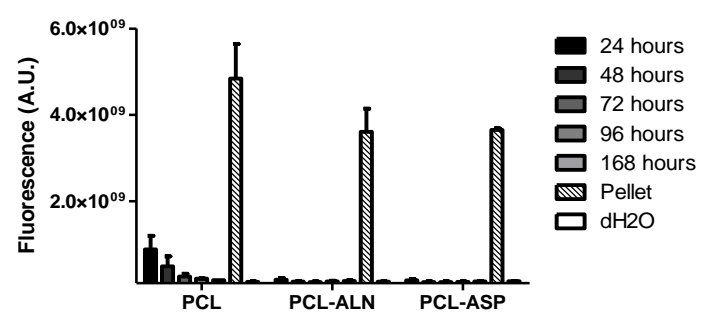

Figure 3A.1 - Detection of IR-780 iodide dye by near infra-red fluorescent imaging.

A. Near infra-red fluorescent signals originating from supernatants and microsphere pellets after 7 days of incubation.

B. Near infra-red fluorescent signal quantification of IR-780 iodide $(n=3)$. 


\section{Microsphere retention in femoral head defects}

Figure 3A.2 shows the fluorescent signals originating from microspheres retained in drilled hole femoral head defects after 7 days. Differences between the fluorescence signals from the PCL microspheres and PCL-ALN or PCL-ASP microspheres with bone targeting properties are non-significant $(p>0.05)$. This observation could be since microspheres were injected deep within the defects, which was an approximately $5 \mathrm{~mm}$ deep cavity into the trabecular bone. Due to subsequent bleeding from the femoral defect, the defects were covered with hemostatic agent (Bone wax) directly after administration of microspheres to prevent rapid wash-out. This could be the reason of the high amount of non-functionalized PCL microspheres in the femoral defect.

A.

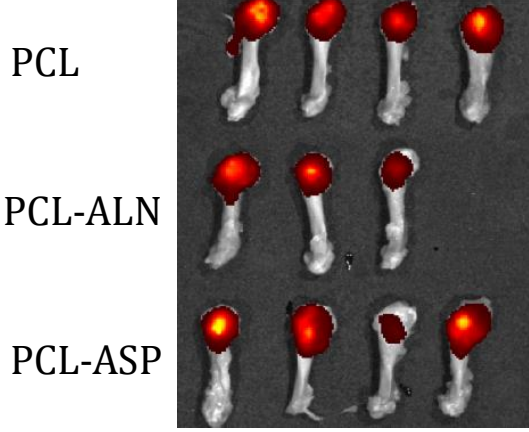

B.

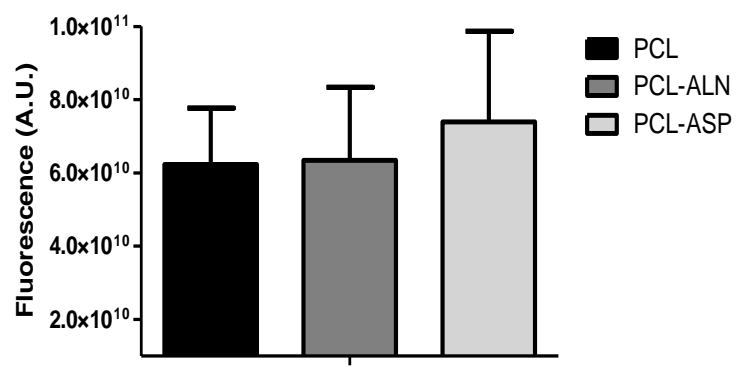

Figure 3A.2 - Retention of microspheres inside rat femoral head defects.
A. Fluorescent imaging of IR-780 iodide loaded microspheres remaining inside rat femoral head defects after 7 days.
B. Quantification of a standardized region of interest localized at the defect site ( $\mathrm{n}$ $=3$ or 4$)$. 


\section{Treatment of $S$. aureus Xen-29 infected femoral defects}

Figure 3A.3 shows bioluminescent signals from Xen-29 present in femoral head defects 14 days after a single administration of microspheres or a vehicle control. As no significant bone binding effect was observed for PCLALN and PCL-ASP microspheres in vivo, only GM-AOT loaded PCL microspheres were tested in this initial treatment experiment to minimize animal subjects. Bioluminescent signals on the femoral heads are only observed in 2/5 femurs while in comparison to the treatment group $0 / 5$ femurs (A.). Due to the relatively high sensitivity threshold of the imaging device, bacterial quantification in homogenized tissues show that all femoral defects remained infected independently of the treatment group. On average an $81 \%$ reduction in CFU is observed when comparing the treatment group with the vehicle control, indicating a slight effect of the antibiotic loaded PCL (B.). Further optimization of the PCL-ALN or PCL-ASP microspheres is expected to further increase antibiotic delivery to the bone tissue, potentially yielding stronger CFU reductions.
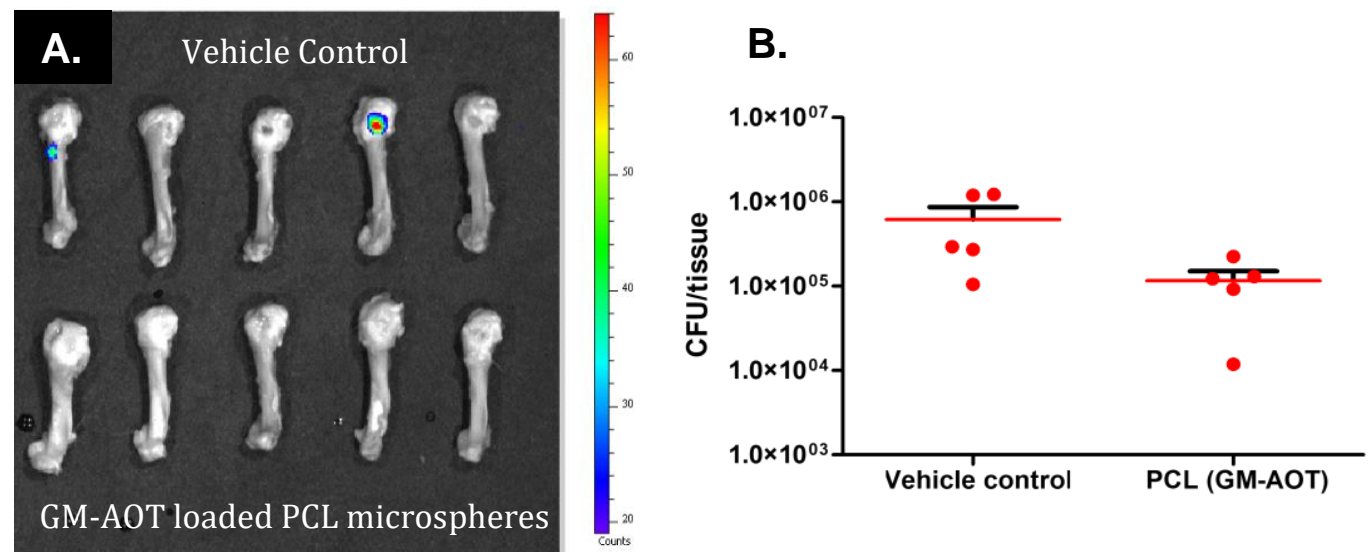

Figure 3A.3 - Quantification of a 14-day old $S$. aureus Xen-29 infection, 7 days after local administration of GM-AOT loaded PCL microspheres or a vehicle control. A. Bioluminescent imaging of $S$. aureus Xen-29.

B. CFU quantification after femoral head homogenization. 


\section{Conclusion}

This appendix reports in vivo preclinical studies on the bone targeting capacity of ALN or ASP functionalized PCL microspheres and the antimicrobial effect of GM-AOT loaded PCL microspheres in a rat bone infection model. Additionally, the anti-fouling and bactericidal properties of functionalized PEEK surfaces in a murine subcutaneous infection model is also reported. PCL-ALN and PCL-ASP microspheres did not show significant improved retention in the rat femoral defect in comparison to pristine PCL microspheres, which differ from the in vitro results, Chapter 3 and 4. Relative high retention of non-functionalized PCL microspheres in the femoral defect most likely originates from the model set-up, in which the bone defect was covered with a hemostatic to prevent excessive bleeding. Optimization of the animal model is necessary to make further conclusions about in vivo bone binding potential of PCL-ALN and PCL-ASP microspheres.

\section{References}

1 Andes, D. R. \& Lepak, A. J. In vivo infection models in the pre-clinical pharmacokinetic/pharmacodynamic evaluation of antimicrobial agents. Current Opinion in Pharmacology 36, 94-99, doi:https://doi.org/10.1016/j.coph.2017.09.004 (2017).

2 Reizner, W. et al. A systematic review of animal models for Staphylococcus aureus osteomyelitis. Eur Cell Mater 27, 196-212, doi:10.22203/ecm.v027a15 (2014).

3 Rotman, S. G. et al. Development of bone seeker-functionalised microspheres as a targeted local antibiotic delivery system for bone infections. Journal of Orthopaedic Translation 21, 136-145, doi:https://doi.org/10.1016/i.jot.2019.07.006 (2020). 


\section{Chapter 4}

\section{Bone targeted antibiotic delivery by poly(aspartic acid) functionalized poly( $\epsilon$-caprolactone) microspheres}

S.G. Rotman ${ }^{1,2}$, T.F. Moriarty ${ }^{1}$, D.W. Grijpma ${ }^{2}$, D. Eglin ${ }^{1}$ and

O. Guillaume ${ }^{1}$

${ }^{1}$ AO Research Institute Davos, Davos Platz, Switzerland

${ }^{2}$ Department of Biomaterials Science and Technology, Faculty of Science and Technology, University of Twente, Enschede, The Netherlands

Published in Pharmaceutics 12(9) (2020) 885 


\section{Abstract}

Bone infection is a feared complication for patients with surgically fixed bone fractures and local delivery of antibiotics plays an adjunctive role in prophylaxis and treatment of these infections. Recent studies have indicated that bacteria, particularly Staphylococcus aureus, are able to penetrate bone tissue through micron-sized canaliculi and evade both systemic and currently available local antibiotic treatments. Targeting bacteria within the bone requires highly efficient and specific delivery of antimicrobials to the infected bone tissue. In this work, a biodegradable microsphere carrier loaded with antibiotics and with specific affinity to bone mineral was developed. Two widely used antibiotics, i.e. Gentamicin-AOT (GM-AOT) and Ciprofloxacin (CF) were embedded in poly ( $\epsilon$-caprolactone) (PCL) microspheres fabricated by oil-in-water emulsion techniques with carboxylated poly(vinyl alcohol) (cPVA) as surfactant. The carboxylic acid groups present at the PCL/cPVA microsphere surface were functionalized with aspartic acid oligomers (ASP) to endow bone targeting properties. We report on the cPVA synthesis, microsphere formulation and antibiotic loading of the PCL/cPVA-ASP microspheres. Once loaded with antibiotics, the PCL/cPVA-ASP microspheres show sustained release of its antibiotic load and can inhibit bacterial growth in vitro for up to 6 days. PCL/cPVA-ASP microspheres show enhanced affinity to mineralized substrates compared to nonfunctionalized PCL/cPVA microspheres. These findings support further development of the bone targeting antibiotic carrier materials for potential treatment of persistent bone infections. 


\section{Introduction}

In orthopaedic surgery, osteomyelitis $(\mathrm{OM})$ is a dreaded complication that can affect up to $27 \%$ of patients with bone fractures [1]. It is generally accepted that a rigorous debridement of infected bone combined with a local and systemic antibiotics is necessary to achieve the highest chance of successful treatment outcome. Nonetheless, for approximately $30 \%$ of patients that undergo such extensive therapies, infection re-emerges within 12 months [2]. Gram-positive Staphylococcus aureus (S. aureus) [3] and Gramnegative Escherichia coli (E. coli) [4] are two main pathogens involved in adult OM. It has been recently shown that $S$. aureus has the ability to deform and penetrate (sub-)micron structures in vitro [5] and this was also observed microscopically in clinical bone biopsies [6]. This phenomenon is expected to contribute to the high recurrence rate of $\mathrm{OM}$ as bacteria residing in bone canaliculi may evade local and systemic antibiotic therapies without causing tissue necrosis and so may easily be missed during surgical debridement procedures.

The presence of bacteria deep within the bone necessitates more efficient antimicrobial delivery strategies. One approach is the direct functionalization of the antibiotic molecules with bone targeting groups, forming a bone targeting prodrug of the active antibiotic [7,8]. Despite straightforward synthesis strategies, there are some downsides to the prodrug approach [9]. The prodrug must show appropriate and controlled conversion into its therapeutically active parent drug and from a translational perspective, the prodrug would need to undergo renewed approval by regulatory institutions before clinical trials can be considered. 
An alternative strategy for enhanced local antibiotic delivery is to load antibiotics into carrier constructs that can release the drug payload in the vicinity of the infection site over a suitable period [10]. For several decades, antibiotic loaded bulk materials like poly(methylmethacrylate) (PMMA) and collagen have been used as carrier materials in orthopaedic surgery and have improved post-operative outcomes significantly [11]. Due to the nonbiodegradable nature of PMMA, a second surgical procedure is needed to retrieve these materials after antibiotic release. Alternatively, collagen carriers (e.g. GENTA-COLL ${ }^{\circledR}$ and Collatamp ${ }^{\circledR G}$ ) are biodegradable, but most of these materials are characterized by their very rapid release of antibiotic load [12]. Some other biodegradable bulk constructs can offer a more sustained release of antibiotic loads, for example poly(trimethylene carbonate) materials, and remove the need for a extraction surgery [13,14]. However, a common feature with all these bulk materials is the inability to localize itself consistently at the bone interface. Antimicrobials loaded within micro or nanoparticles might offer an advantage in this regard [15].

Several attempts have been made to design micro- and nanoparticles for sustained antibiotic delivery $[16,17]$, in which delivery systems were endowed with bone affinity through incorporation of bisphosphonate groups [18]. We previously reported on an antibiotic delivery system consisting of Alendronate (ALN) functionalized poly( $(\epsilon$-caprolactone) (PCL) microspheres in which GM-AOT was embedded in the PCL polymer matrix [18]. However, ALN is known to inhibit osteoclast formation and bone mineral resorption [19] with several ALN-related clinical side effects reported such as bisphosphonate-related osteonecrosis of the jaw (BRONJ) [20], and so alternative bone targeting groups are preferred. Just like ALN, aspartic acid (ASP) oligomers can act as a chelator and interact with divalent 
ions like $\mathrm{Ca}^{2+}$ that are present in inorganic bone matrix, but do not display any secondary effects such as those associated with bisphosphonates. Studies regarding the chelation efficacy of ASP oligomers have reported that oligomers of six or more ASP monomers show the highest affinity to bone minerals [21]. During an investigation of estradiol-ASP prodrugs, residence time of ASP oligomers at the bone site was shown be prolonged by incorporating D-configurated ASP monomers into the oligomers, because D-ASP is not hydrolysable under physiological conditions in contrast to LASP [21]. ASP oligomers have been used to endow bone affinity to radionuclides [22] and PLGA particles [23]. Implementation of ASP oligomers for bone targeted antibiotic therapy has, to the best of our knowledge, not been reported yet. In this work we aimed to develop an oil/water (O/W) emulsion-based production of antibiotic loaded PCL microspheres with (D-ASP) 6 oligomers grafted on the surface via carboxylated PVA (cPVA). Once produced, we further characterize microsphere size and antibiotic loading efficiency as well as their affinity to hydroxyapatite (HAP). Assessment of elution of two antibiotics from the microspheres and their ability to kill Gram-positive and Gram-negative planktonic bacteria and prevent bacterial growth were also studied. Finally, the advantages of ASP functionalized microspheres as antibiotic delivery systems to bone were examined in terms of establishing high local concentrations at a bone mimicking hydroxyapatite surface.

\section{Materials and methods}

\section{Materials}

Poly(vinyl alcohol) (PVA; $\mathrm{M}_{\mathrm{W}}=30.000 \mathrm{~g} \cdot \mathrm{mol}^{-1} ; 100 \%$ hydrolyzed,), $\operatorname{poly}(\epsilon-$ caprolactone) (PCL; $\left.\quad \mathrm{M}_{\mathbb{W}}=80.000 \quad \mathrm{~g} \cdot \mathrm{mol}^{-1}\right), \quad$ 1-Ethyl-3-(3- 
dimethylaminopropyl)carbodiimide (EDC), N-hydroxysuccinimide (NHS), succinic anhydride, dioctyl sulfosuccinate sodium salt (AOT) and ciprofloxacin (CF) were purchased from Sigma Aldrich, Steinheim, Germany. Gentamicin sulphate, 4-Dimethylaminopyridine (4-DMAP), ethanol, 2-propanol, acetone and dichloromethane (DCM) were purchased from Roth (Karlsuhe, Germany). D-(Aspartic acid)6 oligomers (ASP) were synthesized on demand by GenScript (Leiden, the Netherlands). Tryptin Soy Agar (TSA) plates were purchased from Oxoid AG (Basel, Switzerland). Osteoassay plates were acquired from Corning (Amsterdam, the Netherlands). The bacterial strains used were methicillin susceptible $S$. aureus JAR 890 (CCOS, Wädenswill, Switzerland) and E. coli O76:H51 (CCOS, Wädenswill, Switzerland).

\section{Hydrophobic Gentamicin (GM-AOT) synthesis}

GM-AOT was synthesized as previously reported by ter Boo et al. [14] In short, equal volumes of $0.40 \% \mathrm{w} / \mathrm{v}$ gentamicin sulphate in buffer $(10 \mathrm{mM}$ sodium acetate, $\mathrm{KCl}$ and $\mathrm{CaCl}_{2}$ at $\left.\mathrm{pH}=5\right)$ and $1.25 \% \mathrm{w} / \mathrm{v}$ dioctyl sodium sulfosuccinate (AOT) in DCM were mixed by vigorous stirring for 3 hours. The two phases dissociated for 30 minutes and GM-AOT was isolated from the DCM by evaporation of the solvent. Further characterization including Fourier transform infrared spectroscopy (FTIR) and the water solubility of GM-AOT was previously described.[18]

\section{Carboxylated PVA synthesis}

The cPVA synthesis procedure was adapted from Zhang et al. [24] Briefly, PVA (5 gram) was added to $50 \mathrm{~mL} \mathrm{ddH}_{2} \mathrm{O}$ and heated to $90^{\circ} \mathrm{C}$ under magnetic stirring using an oil bath to allow for complete solubilization of 
PVA. The temperature of the PVA solution was cooled to $65^{\circ} \mathrm{C}$ and $1.39 \mathrm{~g}$ 4-DMAP was added under moderate stirring. After 1 hour, 11.36 gram of succinic anhydride was added to the PVA solution and stirred at $65^{\circ} \mathrm{C}$ for 24 hours. The reaction was cooled to room temperature and the polymers were precipitated in $500 \mathrm{~mL}$ acetone and washed a second time in $200 \mathrm{~mL}$ acetone. The polymers were dried overnight under reduced pressure at a temperature of $40^{\circ} \mathrm{C}$. The carboxylated PVA (cPVA) product was analyzed by nuclear magnetic resonance $\left(\mathrm{H}^{1}-\mathrm{NMR}\right)$ in $\mathrm{D}_{2} \mathrm{O}$ solvent with a field strength of 500 $\mathrm{MHz}$ to assess the degree of carboxylation.

\section{PCL/cPVA microsphere fabrication}

$500 \mathrm{mg}$ PCL was dissolved in $5 \mathrm{~mL}$ DCM. When drug was incorporated, 125 $\mathrm{mg}$ of GM-AOT, CF or pyrene was added to the PCL solution. For formulations containing CF, $1 \mathrm{~mL}$ of acetic acid was added as a miscible solvent in order to solubilize the antibiotic. $100 \mathrm{~mL}$ of $1 \%$ aqueous cPVA solution that acted as a surfactant, was $\mathrm{pH}$ adjusted to 8.0 with $1 \mathrm{M} \mathrm{NaOH}$ to ensure a favorable environment for the carboxylic acid groups of cPVA to be in a deprotonated state, in which they act as better surfactants. The polymer solution was added dropwise, under a vortex, to $10 \mathrm{~mL}$ of the cPVA solution over a period of approximately 1 minute. This pre-emulsion was sonicated using a sonication probe (Bandelin Sonopuls GM70, Berlin, Germany) for 3 bursts of 30 seconds and finally added to the remaining 90 $\mathrm{mL}$ of cPVA solution. The DCM could evaporate under ambient conditions with mild magnetic stirring for 4 hours. The solidified microspheres were filtered with a cell-strainer (mesh size of $70 \mu \mathrm{m}$ ) in order to remove agglomerates and washed twice in double distilled water $\left(\mathrm{ddH}_{2} \mathrm{O}\right)$ to remove 
cPVA that remained in solution. The PCL/cPVA microspheres were

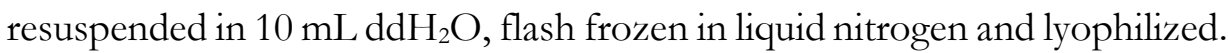

\section{PCL/cPVA microsphere surface functionalization with ASP}

An EDC/NHS solution was made by dissolving $100 \mathrm{mg}$ EDC and $100 \mathrm{mg}$ NHS in $10 \mathrm{~mL}$ MES-buffer at a $\mathrm{pH}$ of 5.5. The lyophilized PCL/cPVA microspheres $(100 \mathrm{mg})$ were fully resuspended in $10 \mathrm{~mL}$ EDC/NHS solution by probe sonication. EDC/NHS activation of the carboxylic acid groups on the microspheres was allowed for 45 minutes at $4^{\circ} \mathrm{C}$. The microspheres were centrifugated and washed with $\mathrm{ddH}_{2} \mathrm{O}$ to remove excess EDC/NHS. Next, the microspheres were dispersed in a $10 \mathrm{mg} / \mathrm{mL}$ ASP oligomer aqueous solution at neutral $\mathrm{pH}$ and the dispersion was stirred mildly for 2 hours at room temperature. Finally, the functionalized microspheres were collected by centrifugation and washed with $\operatorname{ddH}_{2} \mathrm{O}$. The PCL/cPVA-ASP microspheres were flash frozen in liquid nitrogen and lyophilized. A schematic of the final microsphere product is presented in Figure 4.1. 


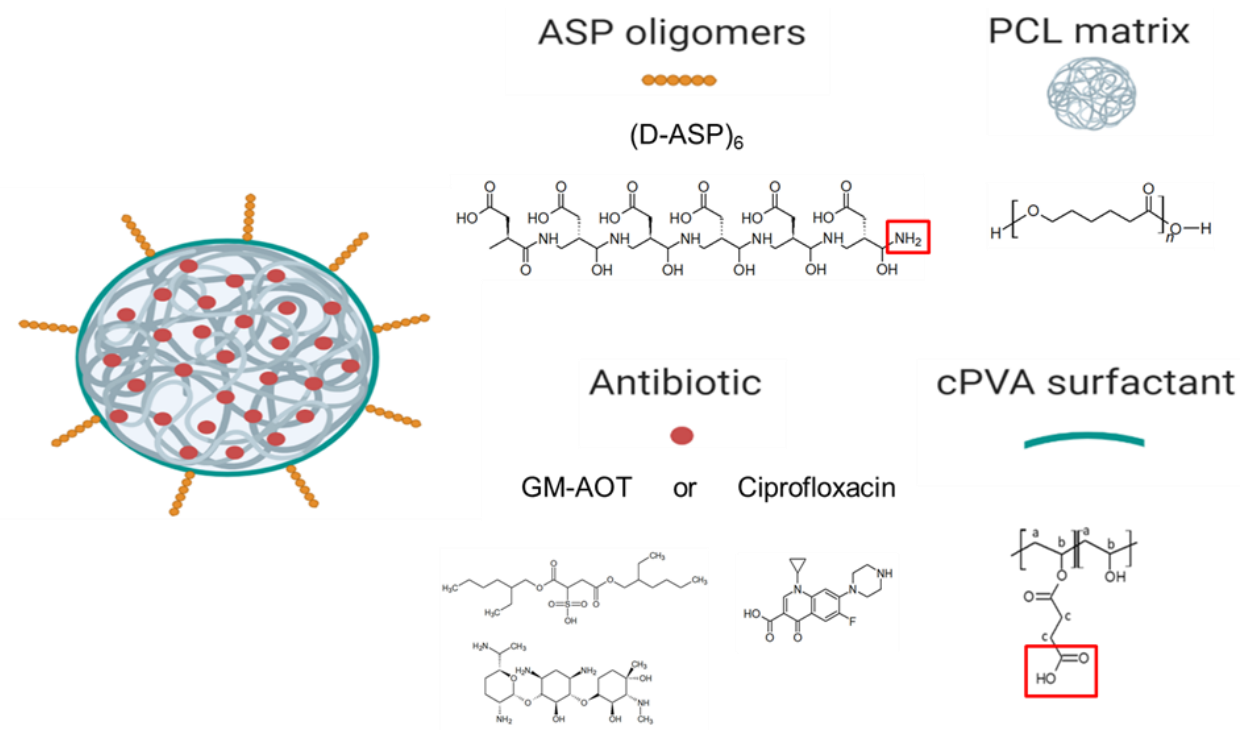

Figure 4.1 - Schematic of PCL/cPVA-ASP microspheres loaded with antibiotic. PCL was used as a biodegradable material for the matrix of the microspheres. The antibiotic CF and the ion-counter ion complex GM-AOT were embedded in the PCL microspheres. ASP oligomers are conjugated to the microsphere cPVA surfactant through EDC/NHS conjugation of the carboxylic acids of cPVA and the primary amine group of the N-terminus of ASP (both boxed in red).

Scanning electron microscopy (SEM) of PCL/cPVA-ASP microspheres

Freeze-dried PCL-cPVA-ASP microspheres loaded with GM-AOT or CF were dispersed in ethanol and $40 \mu \mathrm{L}$ was deposited on scanning electron microscopy (SEM) specimen mounts containing with an adhesive tape. After evaporation of the ethanol, $10 \mathrm{~nm}$ layer of $\mathrm{Au} / \mathrm{Pd}$ was deposited on the samples by sputter-coating. SEM imaging was performed immediately after on a field emission scanning electron microscope (S-4700, Hitachi, Zürich, Switzerland) with a beam voltage of $1.0 \mathrm{keV}$. The microspheres sizes were measured from SEM images using standard Axiovision4 software.

Antibiotic encapsulation efficiency and antibiotic release from PCL/cPVA-ASP microspheres 
PCL/cPVA-ASP microspheres loaded with GM-AOT or CF were disrupted by dispersing the microspheres in $0.1 \mathrm{M} \mathrm{NaOH}$ solution for several hours. Solutions containing the GM-AOT load were subsequently analyzed by complexation with o-phthaldialdehyde reagent, followed by a adsorption measurement $(\lambda=332 \mathrm{~nm})$ using a spectrophotometer (MultiskanGo, Thermo Scientific) [25]. CF encapsulation was determined by high pressure liquid chromatography (HPLC) analysis using a mobile phase of $2 \%$ Acetic acid / Acetonitrile (84:16) under a flow rate of $1 \mathrm{ml} / \mathrm{min}$ and a detection wavelength of $280 \mathrm{~nm}$. To assess the drug quantity embedded inside the microspheres, the embedding efficacy ( $\mathrm{EE} \%$ ) can be determined following equation 1 . The drug load (DL\%) of the microspheres can be calculated with equation 2 .

$$
\begin{aligned}
& E E \%=W_{D M} / W_{D A} * 100 \% \\
& D L \%=W_{D M} / W_{T} * 100 \%
\end{aligned}
$$

With $\mathrm{W}_{\mathrm{DM}}$ as the weight of the drug embedded in the microsphere as determined by HPLC or colorimetric o-phthaldialdehyde assay, $\mathrm{W}_{\mathrm{DA}}$ as the weight of the drug added to the $\mathrm{O} / \mathrm{W}$ emulsion during particle fabrication and $\mathrm{W}_{\mathrm{T}}$ as the total weight of the drug loaded microsphere sample. All EE\% and DL $\%$ values were determined by averages of technical triplicates $(n=3)$.

To establish antibiotic release profiles, accurately weighed quantities $(10 \mathrm{mg})$ of microspheres were dispersed in $1 \mathrm{~mL}$ PBS by probe sonication. The dispersions were centrifuged at 1, 3 and 5 hours and 1, 2, 3, 4, 7, 8, 9 and 13 days to allow for collection of the complete supernatant and were replaced with fresh PBS. GM-AOT and CF concentrations in the collected supernatants were determined as described above. 


\section{Antimicrobial effects on planktonic bacteria}

The opacity of bacterial dispersions upon addition of GM-AOT or CF loaded PCL/cPVA-ASP was monitored over 48 hours in order to assess the antimicrobial effect of released GM-AOT or CF. Bacterial suspensions of $S$. aureus and E. coli was initially set at an optical density at 600 nanometer $\left(\mathrm{OD}_{600}\right)$ of 0.5 in PBS. In triplicates, $5 \mathrm{mg}$ of GM-AOT loaded microspheres were added to $1 \mathrm{~mL}$ of $S$. aureus dispersion and $5 \mathrm{mg}$ of $\mathrm{CF}$ loaded microspheres were added to $1 \mathrm{~mL}$ of E. coli dispersion. The samples were gently resuspended and the $\mathrm{OD}_{600}$ was assessed at regular intervals to assess the reduction in $\mathrm{OD}_{600}$ caused by the bactericidal effect of the released antibiotic. Control groups included bacterial dispersions of both bacterial strains without added microspheres and dispersions of GM-AOT and CF loaded microspheres in PBS.

\section{Zone of inhibition of antibiotic loaded PCL/cPVA-ASP microspheres}

PCL/cPVA-ASP microspheres loaded with GM-AOT or CF were dispersed in PBS at a concentration of $10 \mathrm{mg} / \mathrm{mL}$ and bacterial lawns of $S$. aureus or E. coli were streaked on TSA plates according to the Kirby-Bauer method. In the center of the TSA plate, $100 \mu \mathrm{L}$ of the microspheres dispersions were carefully pipetted on a sterile blanc paper disc (Sensi-disc, BD Biosciences, Franklin Lakes, NJ, USA) in triplicate. GM-AOT loaded microspheres were tested against $S$. aureus bacterial lawns while CF loaded microspheres were tested on E. coli bacterial lawns. The plates were incubated overnight at $37^{\circ} \mathrm{C}$ and $5 \% \mathrm{CO}_{2}$ and the inhibition diameters were measured. The microsphere loaded discs were transferred to a freshly streaked TSA plate and incubated for another 24 hours followed by zone of inhibition (ZOI) measurements. This process was repeated daily until no ZOI was observed. 


\section{PCL/cPVA-ASP affinity with hydroxyapatite surfaces}

Pyrene loaded PCL/cPVA-ASP microspheres were incubated in an Osteoassay 96 well plate containing thin HAP films on the bottom of the wells. After 1 hour, the wells were washed three times with $\mathrm{ddH}_{2} \mathrm{O}$. Between each wash, the well plate was measured using a fluorescence plate reader (Viktor ${ }^{3}$, Perkin Elmer, Schwerzenbach, Switzerland) with a Hoechst filter $\left(\lambda_{\mathrm{ex}} / \lambda_{\mathrm{em}} \sim 353 \mathrm{~nm} / 483 \mathrm{~nm}\right)$. A standard containing a known weight of Pyrene loaded PCL/cPVA-ASP microspheres was measured after every washing step to account for any leeching of the pyrene out of the microspheres. In order to visualize the microspheres on the HAP films, the Osteoassay 96 well plates were imaged using a fluorescent microscope (EVOS FL Auto 2, Thermo Fisher Scientific, Basel, Switzerland), again implementing the Hoechst filter settings.

\section{Zone of inhibition around hydroxyapatite granules incubated with PCL/cPVA-ASP}

HAP granules $(n=4)$ with diameters between 5 and 10 millimeters were incubated for 1 hour in a GM-AOT or CF loaded PCL/cPVA or PCL/cPVA-ASP microsphere dispersions at a concentration of $5 \mathrm{mg} / \mathrm{mL}$ under mild rocking conditions. The HAP granules were washed in PBS and were placed centrally on a TSA plate that was streaked with a $S$. aureus or E. coli bacterial lawn prepared as described above. The HAP granules were slightly pressed into the agar to ensure contact between the granule and the agar layer. After overnight incubation at $37^{\circ} \mathrm{C}$, the average zone of inhibition around the HAP granule was calculated from 4 measurements in different directions. Due to the varying sizes of the HAP granules, the distance from the granule surface to the inhibition front was measured, instead of the total 
diameter of the inhibition zone. The granules were transferred to a freshly streaked agar plate, in order to assess the zone of inhibition established by antibiotic released at day 2 . This process was repeated daily until an inhibitory zone could no longer be observed. 


\section{Results}

\section{${ }^{1} \mathrm{H}-\mathrm{NMR}$ analysis of cPVA}

After the carboxylation process of PVA, the cPVA product was analyzed by ${ }^{1} \mathrm{H}$ NMR spectroscopy of which the spectra can be seen in Figure 4.2. Successful grafting is presented by a peak at $2.56 \mathrm{ppm}$, caused by the protons of the methylene groups of ring-opened succinic anhydride (succinic acid) grafted on the PVA molecular backbone. The degree of substitution of PVA's hydroxyl groups to succinic acid groups was determined to be 45 mole $\%$, from the peak integration of proton signals of the PVA backbone and the methylene groups of grafted succinic acid.

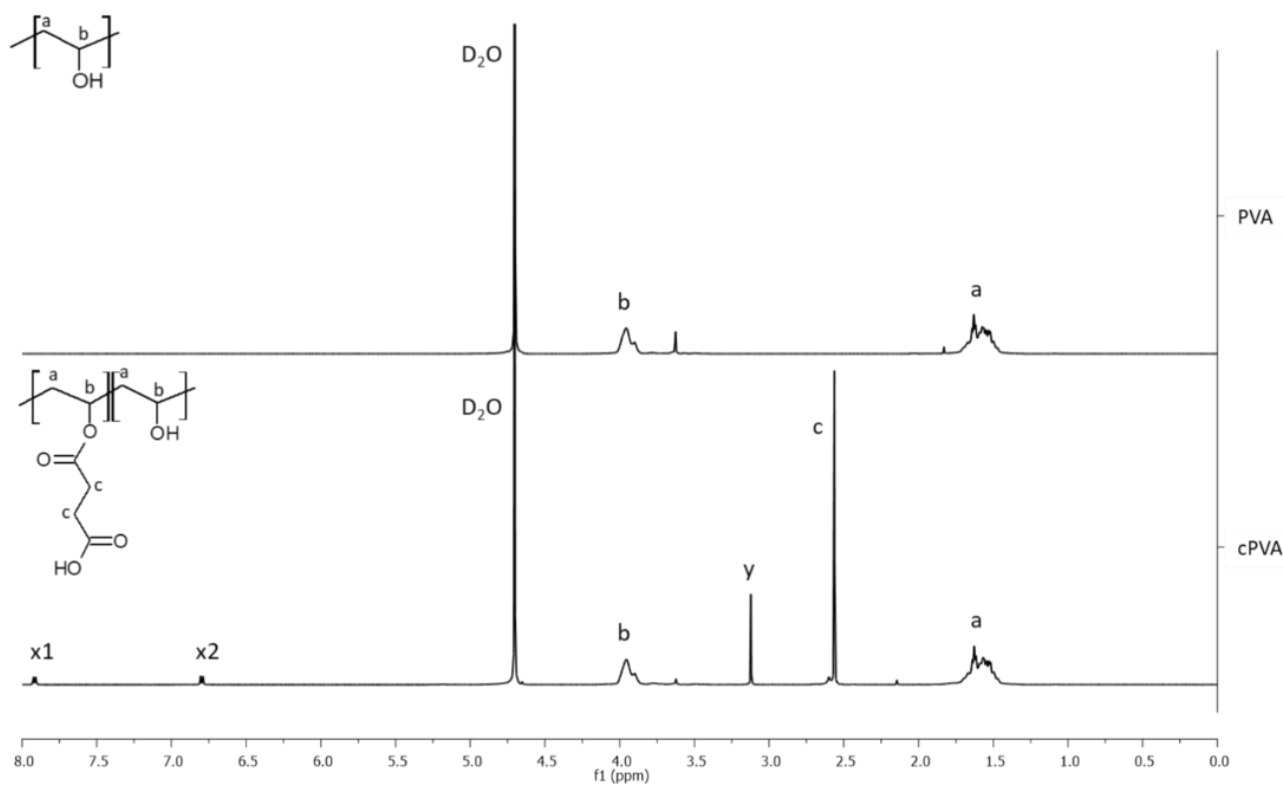

Figure 4.2 - NMR spectra of PVA and cPVA. Poly(vinyl alcohol) (PVA): ${ }^{1} \mathrm{H}$ NMR $\left(\mathrm{D}_{2} \mathrm{O}, 500 \mathrm{MHz}\right): \mathrm{a}=\delta 1.62\left(\mathrm{~m}, 2 \mathrm{H}, \mathrm{CH}_{2}\right), \mathrm{b}=\delta 3.96\left(\mathrm{~m}, 2 \mathrm{H}, \mathrm{CH}_{2}\right)$. Carboxylated poly(vinyl alcohol) (cPVA): ${ }^{1} \mathrm{H}$ NMR $\left(\mathrm{D}_{2} \mathrm{O}, 500 \mathrm{MHz}\right): \mathrm{a}=\delta 1.62\left(\mathrm{~m}, 2 \mathrm{H}, \mathrm{CH}_{2}\right), \mathrm{c}=$ $\delta 2.56\left(\mathrm{~s}, 4 \mathrm{H}, \mathrm{CH}_{2} \mathrm{CH}_{2}\right), \mathrm{b}=\delta 3.96\left(\mathrm{~m}, 2 \mathrm{H}, \mathrm{CH}_{2}\right), \mathrm{x}=4$-DMAP trace peaks, $\mathrm{y}=$ succinic anhydride trace peaks. 


\section{PCL/cPVA-ASP microsphere characteristics}

After PCL/cPVA functionalization with ASP, the microspheres were imaged by SEM. Two representative images of the two groups of microspheres containing GM-AOT and CF can be seen in Figure 4.3A and Figure $4.3 \mathrm{~B}$ respectively. As the fabrication methods are identical, size averages of the microspheres were dependent on the embedded drug, with GM-AOT loaded microspheres having a size average of $6.03 \mu \mathrm{m} \pm 4.05 \mu \mathrm{m}$ $(\mathrm{n}=110)$ while CF loaded microspheres were $1.24 \mu \mathrm{m} \pm 0.76 \mu \mathrm{m}(\mathrm{n}=103)$ in diameter. The EE\% of antibiotics in PCL/cPVA-ASP microspheres were $12.76 \pm 1.78 \%$ for $\mathrm{CF}$ and $23.77 \pm 1.39 \%$ for GM-AOT. The DL $\%$ was 3.19 $\pm 1.53 \%$ for $\mathrm{CF}$ and $4.75 \pm 0.28 \%$ for GM-AOT.
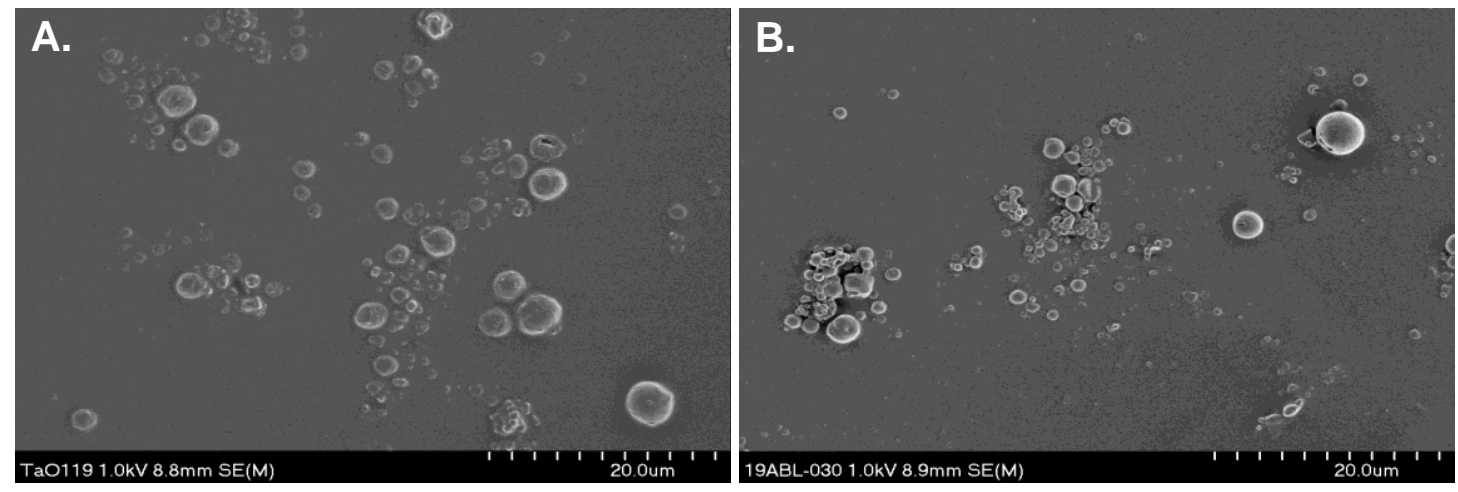

Figure 4.3 - A. PCL/cPVA-ASP microspheres with a GM-AOT drug load. B. PCL/cPVA-ASP microspheres with a CF drug load. Scalebar equals $20 \mu \mathrm{m}$.

\section{Antibiotic release from PCL/cPVA-ASP microspheres}

Data on the release of CF and GM-AOT from PCL/cPVA-ASP was collected and subjected to various drug release models including the Higuchi model and the Korsmeyer-Peppas model [26]. Both models can be considered as important tools to understand which type of release kinetics 
take place during the elution of drug from the polymeric microspheres. Figure 4 presents the cumulative release of $\mathrm{CF}$ and GM-AOT (Figure 4.4A \& Figure 4.4D) and the same data presented in Higuchi (Fig. 4.4B \& 4.4E) and Korsmeyer-Peppas plots (Figure 4.4C \& 4.4F). Linear trendlines were fitted to the Higuchi and Korsmeyer-Peppas datasets by means of linear regression with the fit equation and $\mathrm{R}^{2}$ reported for each of the graphs.
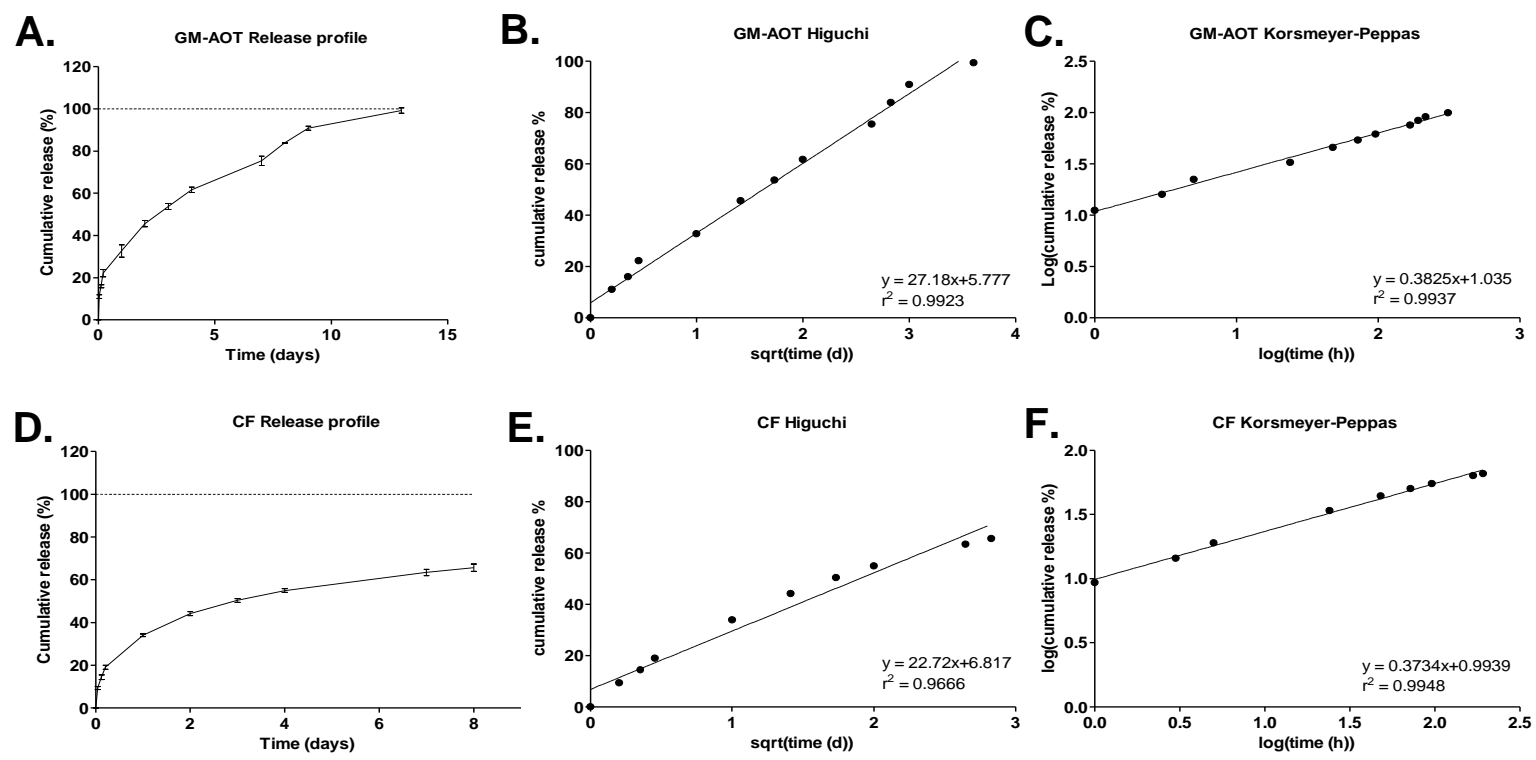

Figure 4.4 - A. Release profile of GM-AOT from PCL/cPVA-ASP microspheres. B. GM-AOT release data represented in a Higuchi plot. C. GM-AOT release data represented in a Korsmeyer-Peppas plot. D. Release profile of CF from PCL/cPVAASP microspheres. E. CF release data represented in a Higuchi plot. F. CF release data represented in a Korsmeyer-Peppas plot.

Figure 4.4A \& Figure 4.4D show the cumulative release of GM-AOT and CF respectively. The release of GM-AOT nearly reached its complete drug load after two weeks. CF release appeared to plateau after 1 week of antibiotic release. In Figure 4.4B \& Figure 4.4E the antibiotic release data was plotted on a Higuchi axis system (square root of time vs. cumulative release), with linear fits with $\mathrm{r}^{2}$ of 0.9923 and 0.9666 respectively. This 
demonstrated that the drug release was predominantly diffusion controlled. The slightly poorer linear fit of the CF release data might be due to the incomplete drug release and plateauing of the release profile at approximately $60 \%$ of cumulative $\mathrm{CF}$ release.

Drug release of GM-AOT and CF from PCL/cPVA-ASP also fits well with the Korsmeyer-Peppas model (Figure 4.3C \& Figure 4.3F; $\mathrm{r}^{2}$ of 0.9937 and 0.9939 respectively) and the value of the slope of the curve fitting (n-value) yields information on how the drug load is released from the polymeric matrix (diffusion, carrier erosion or a combination). When the n-value parameter modeling drug release from spherical materials is 0.43 , it is considered that the drug is released in a Fickian diffusion manner. The nvalues of GM-AOT and CF release reported in this work are 0.38 and 0.37 respectively. An n-value below 0.43 is expected for polydisperse spherical samples [27,28], as is indeed the case in this work. The main characteristics of Fickian diffusion are defined by a slow polymer relaxation and a high diffusion rate of solvent to the interior of the microsphere. Polymers with a glass transition temperature $\left(T_{g}\right)$ below the environmental temperature are often characterized by a Fickian diffusion of the drug from their polymeric matrix [26]. As the $\mathrm{T}_{\mathrm{g}}$ of $\mathrm{PCL}$ is $-60^{\circ} \mathrm{C}$ and the microspheres were incubated at $37^{\circ} \mathrm{C}$, this statement conforms to our findings.

\section{In vitro antimicrobial effects of GM-AOT and CF loaded PCL/cPVA- ASP microspheres}

First, antimicrobial effects were assessed by monitoring the evolution of the $\mathrm{OD}_{600}$ of bacterial dispersions supplemented with GM-AOT and CF loaded PCL/cPVA-ASP microspheres over time and the results are shown in Figure 4.5A. The $\mathrm{OD}_{600}$ of the relevant control groups are shown in the 
supplementary Figure 4.S1. The antibiotics released from the microspheres caused a sharp reduction in optical density of the dispersion. Even though bacterial dispersions at $37^{\circ} \mathrm{C}$ in PBS in absence of antibiotics still causes a mild $\mathrm{OD}_{600}$ decrease due to a lack of bacterial nutrients or bacterial agglomeration (Figure. 4.S1), the $\mathrm{OD}_{600}$ decrease observed in Figure $4.5 \mathrm{~A}$ is evidently much higher and thus caused by the presence of CF or GM-AOT loaded microspheres. In Figure 4.5B, the remaining CFU concentration of the microsphere supplemented bacterial samples and bacterial control samples are presented. A $5 \log _{10}$ reduction of $S$. aureus and a $3.5 \log _{10}$ reduction of E. coli CFU was reported, showing the strong antimicrobial effects of the antibiotics released from PCL/cPVA-ASP microspheres.

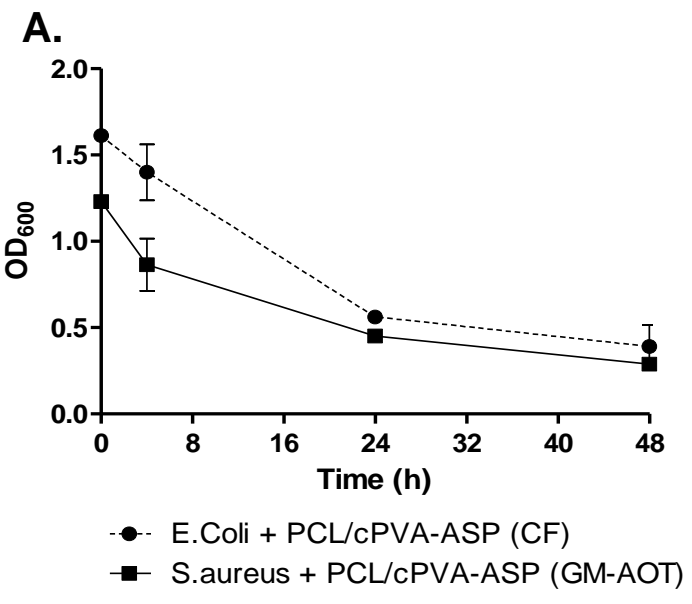

B.

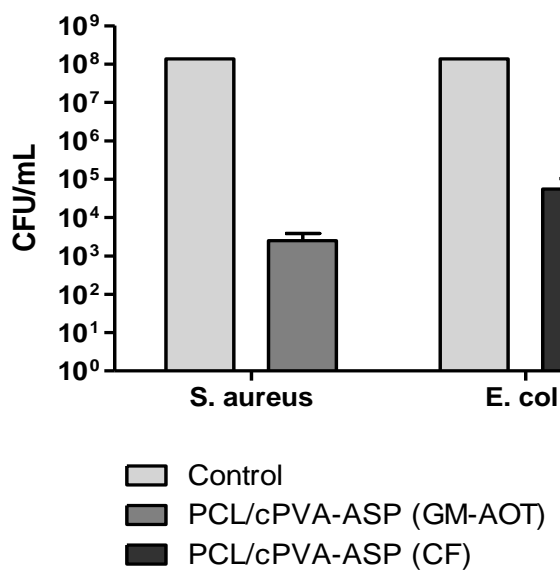

Figure $4.5-$ A. Optical density at $600 \mathrm{~nm}$ of $E$. coli and $S$. aureus bacterial dispersions monitored over 48 hours. B. Remaining CFU concentrations of control bacterial dispersions and bacterial dispersions treated with antibiotic loaded PCL/cPVA-ASP after a 48-hour incubation.

PCL/cPVA-ASP affinity with hydroxyapatite surfaces

Affinity of PCL/cPVA microspheres to HAP surfaces was increased after ASP grafting on the cPVA surfactant layer (Figure 4.6). To quantify 
microsphere presence on HAP surfaces, fluorescent pyrene was embedded in the PCL matrix. Figure 4.6A shows the standard curves of fluorescent signals coming from pyrene embedded in PCL/cPVA and PCL/cPVA-ASP microspheres. The reduced fluorescent signal of PCL/cPVA-ASP compared to PCL/cPVA microspheres can be explained by a loss of pyrene during the ASP functionalization procedure and highlights the importance of establishing two standard curves when assessing PCL/cPVA and PCL/cPVA-ASP fluorescent signals. Figure 4.6B shows the binding efficacy of PCL/cPVA and PCL/cPVA-ASP to HAP surfaces. A significant increase of PCL/cPVA-ASP microspheres present on the HAP surfaces compared to PCL/cPVA microspheres demonstrates that the ASP oligomers endow the microspheres with bone binding properties. Figure 4.6C shows fluorescence microscopic images originating from pyrene loaded PCL/cPVA or PCL/cPVA-ASP on HAP surfaces. Increased intensity of the signal confirms a higher affinity between ASP functionalized microspheres and bone-like inorganic material, corroborating the data from Figure 6B. 
A.

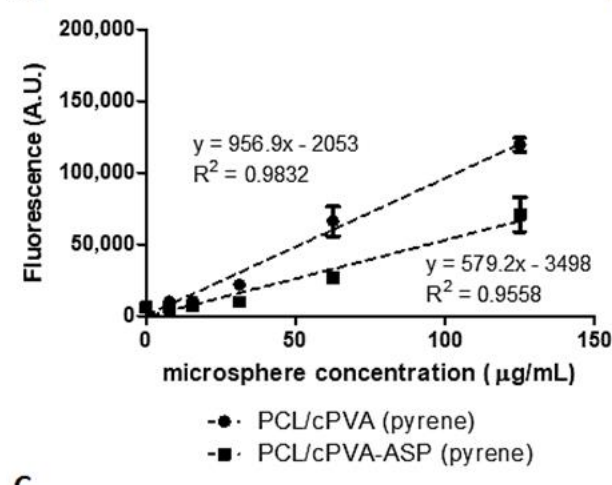

C.

\section{PCL/CPVA}

B.

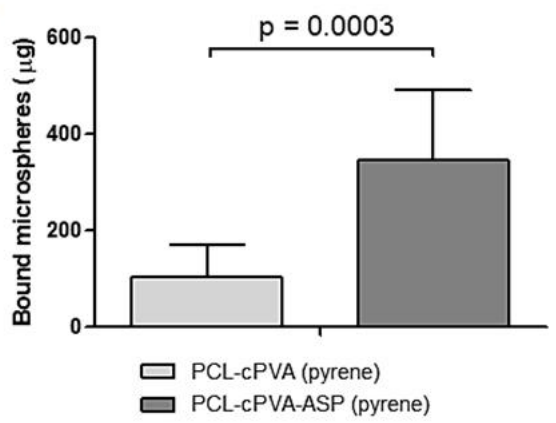

\section{PCL/CPVA-ASP}

$100 \mu \mathrm{m}$

Figure 4.6 - A. Standard curves of the Hoechst absorption by pyrene loaded in PCL/cPVA and PCL/cPVA-ASP microspheres. B. Quantitative assessment of the presence of pyrene loaded microspheres on calcium phosphate surfaces established by standard curve correlation. C. Microscopic images of pyrene loaded in PCL/cPVA and PCL/cPVA-ASP microspheres on calcium phosphate surfaces.

\section{Zone of inhibition established by PCL/cPVA-ASP}

The progression of the ZOI surrounding $1 \mathrm{mg}$ of antibiotic-loaded PCL/cPVA or PCL/cPVA-ASP microspheres is showed in Figure 4.7A. From this figure, it can see that both PCL/cPVA and PCL/cPVA-ASP microspheres show a ZOI for both embedded antibiotics. However, it can be deduced that equal quantities of PCL/cPVA microspheres would establish a larger ZOI for a longer duration compared to PCL/cPVA-ASP that underwent further surface functionalization. This can be expected due to the loss of drug load during the functionalization procedure. The 
combined bone-binding and antimicrobial functions are presented in the form of inhibitory zone measurements of HAP granules that were exposed to suspensions of antibiotic loaded PCL/cPVA or PCL/cPVA-ASP microspheres. Figure 4.6B shows the ZOI established surrounding HAP granules after exposure to PCL/cPVA or PCL/cPVA-ASP. Due to the enhanced affinity of PCL/cPVA-ASP compared to PCL/cPVA, a larger and more persisting ZOI could be observed around the HAP granule for ASP functionalized microspheres with GM-AOT and CF. The microspheres without bone targeting moieties were able to establish an inhibition zone around the HAP granule on the first or second day due to non-specific adherence of small amounts of PCL/cPVA microspheres, matching with the findings observed during our HAP affinity assay (Figure 4.6). However, negligible antimicrobial effects were observed for adhering PCL/cPVA microspheres at later timepoints. In contrast, when microspheres were functionalized with ASP, a larger ZOI diameter could be observed that was observable for up to 4 (GM-AOT) to 6 days (CF). The ZOI observations from Figure 4.7A and 4.7B and the fluorescent imaging from figure 4.6C validate that microspheres with ASP surface functionalization exhibit enhanced affinity to HAP granules and release increased amounts of antibiotic in the vicinity of HAP granules. 

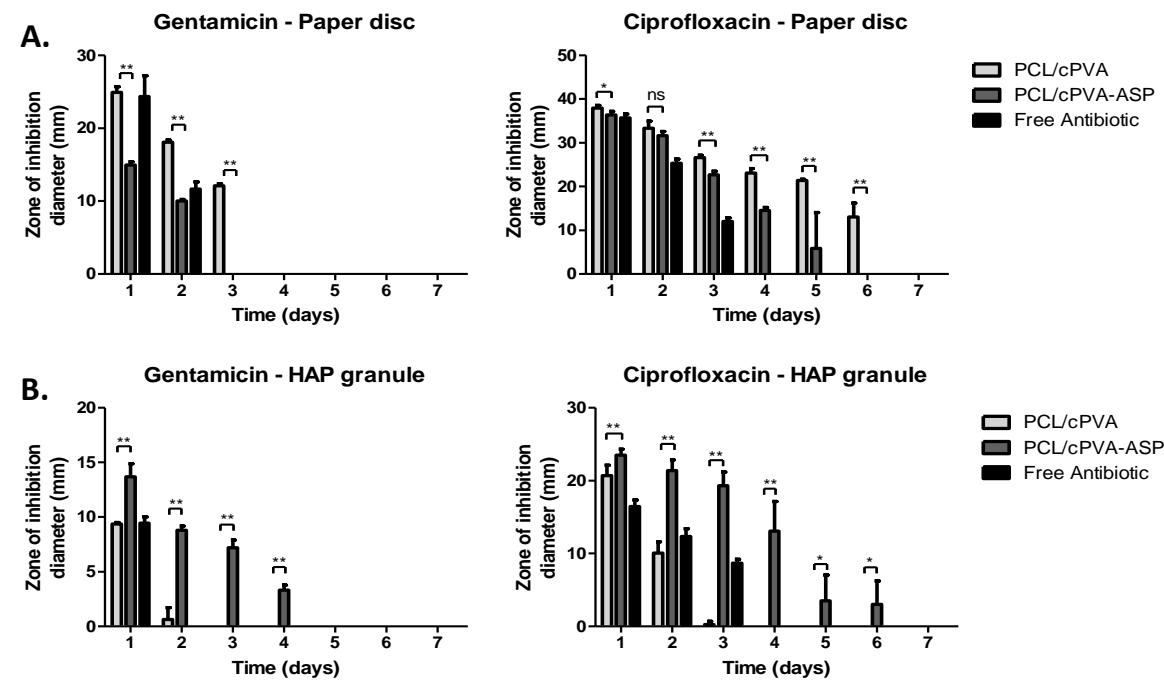

Figure 4.7 - A. Zone of inhibition established by $1 \mathrm{mg}$ of PCL/cPVA or PCL/cPVAASP with antibiotic (GM-AOT, left and CF, right) load on paper discs. B. Zone of inhibition around a HAP granule exposed to GM-AOT or CF loaded microsphere dispersions. (**: $\mathrm{p}<0.01$. ns: no statistical significance $(\mathrm{p}>0.05))$

\section{Discussion}

The relatively high recurrence rate of bone infection despite appropriate therapy suggests that improvements in antibiotic delivery to the infection site are required. Antibiotic carriers currently applied in clinics possess suboptimal properties such as non-biodegradability, incomplete antibiotic release or excessive burst release of antibiotic load. Additionally, many currently available antibiotic carriers come in bulky dimensions, necessitating invasive surgeries while preventing antibiotic release directly at the interface of the bone or even within the bone. In this work we introduced an antibiotic microsphere carrier system that could efficiently bind to calcified materials through interaction between the polymeric antibiotic carrier and calcium ions in HAP and release its antibiotic payload in close vicinity to infected bone mimics. 
In our previous investigations, the bone targeting moiety was conjugated to $\mathrm{COOH}$ groups on the surface of the PCL microspheres, which were generated by alkaline hydrolysis (saponification) of PCL in $0.1 \mathrm{M} \mathrm{NaOH}$ solutions. Due to hydrolytic degradation in presence of the such high $\mathrm{pH}$ levels, this procedure denatured the embedded GM-AOT antibiotic and reduced its antimicrobial capacities. As many other antibiotics do not tolerate such alkaline environments, our current motivation was to adjust the functionalization strategy by including functionalized carboxylated PVA (cPVA) surfactants in order to enrich the surface of the microspheres with $\mathrm{COOH}$ groups. While cPVA has been investigated with other applications in mind (e.g. in relation with the modification of PVA crosslinking properties [29,30]), no reports have been found where cPVA was implemented for drug delivery purposes. Additionally, ASP oligomers were utilized as bone targeting moieties in this work opposed to ALN which showed impairment of osteoclast functionality our previous work [18].

Gentamicin sulphate (GM) and CF were used as they are commonly applied in orthopaedic surgery as a prophylactic agent or as a treatment for infections [31,32]. GM and CF are both active against Gram-positive and Gramnegative bacteria and can be considered as broad-spectrum antibiotics. Due to their broad antimicrobial properties, these antibiotics were considered suitable for incorporation in the developed delivery system. As GM is a hydrophilic antibiotic which is challenging to embed in polymer microspheres fabricated by $\mathrm{O} / \mathrm{W}$ emulsions, we have previously applied a hydrophobic ion pairing method to substitute the sulphate ions of GM with a hydrophobic dioctyl sulfosuccinate counter-ion, resulting in the synthesis of water-insoluble GM-AOT $[14,18]$. This modified antibiotic could be efficiently embedded in hydrophobic matrices and showed similar 
antimicrobial properties compared to GM [18]. Additionally, the antibiotic $\mathrm{CF}$ was also interesting for investigation in bone drug delivery as its molecular structure allows for chelation to divalent metallic ions [33] and can thus potentially interact with $\mathrm{Ca}^{2+}$ after being released from the microspheres. This interaction between $\mathrm{CF}$ and HAP was shown to be reversible at $37^{\circ} \mathrm{C}$ and $\mathrm{CF}$ release from HAP composite materials was monitored in previous studies [34].

Although CF and GM-AOT can kill a broad range of bacteria [35,36], a versatile antibiotic carrier should ideally be able to carry as many different antibiotics as possible to ensure wide clinical applicability. Hydrophobic antibiotics are most efficiently embedded in polymeric microspheres fabricated by $\mathrm{O} / \mathrm{W}$ emulsions, as hydrophilic drug would be dissolved in the voluminous water phase instead of in the droplets of polymer solution. Additionally, hydrophobic drugs are benefiting most from drug delivery systems as their bioavailability is generally low [37]. Solvents involved in the $\mathrm{O} / \mathrm{W}$ emulsions must be carefully selected as they must retain specific physicochemical properties. First, the oil phase solvent needs to dissolve polymer and antibiotic alike. Second, the oil phase solvent needs to be immiscible with the water phase in order to generate emulsions. Finally, the oil phase solvent needs to evaporate in a reasonable rate in order to generate solid polymer microspheres. If necessary, the oil phase could consist of a solvent mixture to optimize its properties to match with the earlier mentioned criteria, but this more complex $\mathrm{O} / \mathrm{W}$ emulsion would require extensive characterization to evaluate differences in solubility parameters and evaporation rates. Many polyesters like PCL and PLGA are soluble in organochlorides (e.g. DCM and chloroform), which generally also have low boiling temperatures that facilitate rapid evaporation. When considering 
antibiotics that can be dissolved in such solvents, we find that compounds such as rifampicin [38], erythromycin [39] and fluoroquinolones (e.g. levofloxacin and ciprofloxacin) [40] are good candidates for loading into polymeric microspheres made by these $\mathrm{O} / \mathrm{W}$ emulsions, underlining the drug load versatility of the presented delivery system.

Even though both GM-AOT and CF loaded microspheres showed bactericidal properties (Figure 4.4), a clear difference in EE\% and DL\% of GM-AOT and CF was observed. Lower CF loading into the microspheres could be due to the lower solubility of CF in the DCM/acetic acid co-solvent system and different miscibility properties in the subsequent emulsification within the aqueous phase. In order to optimize CF encapsulation a $\mathrm{W}_{1} / \mathrm{O} / \mathrm{W}_{2}$ emulsion could be considered, in which the $\mathrm{CF}$ load could be incorporated into the inner aqueous phase consisting of acidic solvents such as diluted acetic acid.

Because ASP oligomers are incorporated in a post-fabrication functionalization strategy, it is important to assess the stability of the drug load under the conditions created in the functionalization process. In our previous work, a relatively strong alkaline environment was established in order to promote alkaline hydrolysis of the PCL chains for $\mathrm{COOH}$ formation [18]. This strategy resulted in reduced activity of the incorporated GM-AOT antibiotics, which diminishes the therapeutic efficacy of the drug delivery system. In this work, $\mathrm{COOH}$ groups were grafted on the PVA surfactants by esterification of the hydroxyl groups with ring opened succinic anhydride (alkaline Shiina esterification) [24]. To increase reaction rate, 4DMAP was used as a nucleophilic catalyst. Due to concerns about the potentially toxic properties of 4-DMAP, other tertiary amine alternatives 
such as 4-Dimethylaminopyridine $\mathrm{N}$-oxide would also be good candidates to catalyze this reaction [41]. However, with the extensive washing of the cPVA product and the presence of cPVA limited to a very thin surface layer on the microspheres, we can assume that trace amount of 4-DMAP is negligible. The $\mathrm{COOH}$ groups could be functionalized with ASP oligomers by EDC/NHS conjugation chemistry under mild conditions that allowed retention of the antimicrobial potential of the loaded antibiotics as evidenced in Figure 4.4.

Comparative literature on drug delivery through peptide-based bone targeting is limited. Jiang et al. has performed a promising study on PLGAPEG nanoparticles (NPs) functionalized with ASP and glycine (GLY) oligomers of six repeating units [23]. The oligomers were labeled with fluorescein isothiocyanate (FITC) in order to quantify the NPs binding affinity. Dispersions of ASP functionalized NPs were exposed to HAP containing hydrogels and NPs remaining in dispersion were quantified by light absorption measurements. Results showed that $85 \%(85 \mu \mathrm{g})$ of ASP

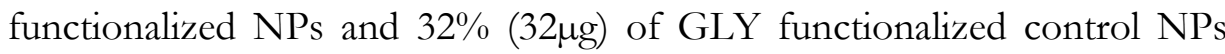
attached to the HA-hydrogel. This ratio between non-specific interaction of the GLY functionalized NPs and targeted interaction of the ASP functionalized NPs (1:2.65 ratio) is comparable to the results shown in Figure 4.5, where $103 \mu \mathrm{g}$ of PCL/cPVA and $346 \mu \mathrm{g}$ of PCL/cPVA-ASP (1: 3.36 ratio) was quantified on HAP surfaces. As no information of the ASP configuration (D- or L-) was given by Jiang et al. it could be argued that the higher affinity ratio to calcified materials that we reported could be due to the non-hydrolysable D-ASP. 
In a final experiment, the positive effect of the bone-binding surface was demonstrated via the larger and long-lasting inhibitory zone measurements of antibiotic loaded PCL/cPVA-ASP microspheres in comparison to PCL/cPVA microspheres loaded on HAP granules. Interestingly, even though CF encapsulation in PCL/cPVA-ASP microspheres $(\mathrm{EE} \%=12.76$ $\pm 1.78 \%$ ) was lower than the encapsulation of GM-AOT (EE $\%=23.77 \pm$ $1.39 \%$ ), the ZOI that resulted from CF release was higher and persisted longer. This observation can be explained due to variability in minimal inhibitory concentration (MIC) between to two antibiotics and S. aureus and E. coli bacterial strains. Additionally, the molecular structure of CF allows for chelation through its carboxylic acid and ketone groups [42]. The release of chelated CF from a HAP/PCL nanocomposite substrate was previously reported and large quantities of CF (reported in absorbance values, but not correlated to a CF dosage) were detected in the release media over the course of 4 days at $37^{\circ} \mathrm{C}$ [34]. Thus, it can be hypothesized that the $\mathrm{CF}$ intrinsic chelating ability to HAP combined with the sustained CF release from the PCL/cPVA-ASP microspheres bonded to the HAP, further enhance the local concentration of antibiotic at the bone-like material surface.

This work reports on a new approach towards carboxylation of microparticulate systems, implementing functionalized cPVA surfactants that facilitate further grafting of functional surface moieties. We believe that this strategy is of particular interest to a multitude of applications in targeted therapeutics. Specifically, the PCL/cPVA-ASP microspheres introduced in this work show to be a versatile drug delivery platform for antibiotic delivery to bone that, once further assessed in pre-clinical infection models, is open for clinical translation. 


\section{Conclusion}

In this work we set out to develop a bone targeting antibiotic delivery system that was able to release antibiotic load in a sustained manner at the interface of calcified materials. The slow release of GM-AOT and CF antibiotics could reduce the optical density of bacterial suspensions and reduce bacterial titers by $5 \log _{10}$ and $3.5 \log _{10}$ respectively. PCL/cPVA-ASP microspheres showed a 3.36-times increased affinity to bone-mimicking surfaces compared to PCL/cPVA microspheres, ensuring that antibiotic release out of the microspheres occurs at the bone surface. Finally, we showed that PCL/cPVA-ASP microspheres could bind to HAP granules, surrogate for the inorganic bone matrix, and that a larger inhibition zone surrounding the granules could be observed for several days, in contrast to antibiotic loaded microspheres without ASP functionalization. In future studies, an assessment of the in vivo antimicrobial properties of the injectable bone targeting antibiotic loaded microspheres must be performed in a relevant animal OM model to confirm these in vitro findings.

\section{Acknowledgements}

This work was supported by the AOTrauma Clinical Priority Program Bone Infection and by the AO Foundation. Grant number: AR2015-04. Issued in Switzerland. 


\section{References}

1 Pollak, A. N. et al. The relationship between time to surgical debridement and incidence of infection after open high-energy lower extremity trauma. $J$ Bone Joint Surg Am 92, 7-15, doi:10.2106/JBJS.H.00984 (2010).

Tice, A. D., Hoaglund, P. A. \& Shoultz, D. A. Outcomes of osteomyelitis among patients treated with outpatient parenteral antimicrobial therapy. The American Journal of Medicine 114, 723-728, doi:10.1016/S0002-9343(03)00231-6 (2003).

Hogan, A., Heppert, V. G. \& Suda, A. J. Osteomyelitis. Archives of orthopaedic and trauma surgery 133, 1183-1196, doi:10.1007/s00402-013-1785-7 (2013).

Carek, P. J., Dickerson, L. M. \& Sack, J. L. Diagnosis and management of osteomyelitis. American family physician 63, 2413-2420 (2001).

de Mesy Bentley, K. L. et al. Evidence of Staphylococcus Aureus Deformation, Proliferation, and Migration in Canaliculi of Live Cortical Bone in Murine Models of Osteomyelitis. J Bone Miner Res 32, 985-990, doi:10.1002/jbmr.3055 (2017).

de Mesy Bentley, K. L., MacDonald, A., Schwarz, E. M. \& Oh, I. Chronic Osteomyelitis with Staphylococcus aureus Deformation in Submicron Canaliculi of Osteocytes: A Case Report. JBJS Case Connect 8, e8-e8, doi:10.2106/JBJS.CC.17.00154 (2018).

Albayati, Z. A. F. et al. Novel Bone-Targeting Agent for Enhanced Delivery of Vancomycin to Bone. Antimicrobial Agents and Chemotherapy 60, 1865, doi:10.1128/AAC.01609-15 (2016).

Sedghizadeh, P. P. et al. Design, Synthesis, and Antimicrobial Evaluation of a Novel Bone-Targeting Bisphosphonate-Ciprofloxacin Conjugate for the Treatment of Osteomyelitis Biofilms. J Med Chem 60, 2326-2343, doi:10.1021/acs.jmedchem.6b01615 (2017).

9 Rautio, J., Meanwell, N. A., Di, L. \& Hageman, M. J. The expanding role of prodrugs in contemporary drug design and development. Nature Reviews Drug Discovery 17, 559-587, doi:10.1038/nrd.2018.46 (2018).

10 Rotman, S. G. et al. Drug delivery systems functionalized with bone mineral seeking agents for bone targeted therapeutics. Journal of Controlled Release 269, 8899, doi:https://doi.org/10.1016/i.jconrel.2017.11.009 (2018).

11 ter Boo, G.-J. A., Grijpma, D. W., Moriarty, T. F., Richards, R. G. \& Eglin, D. Antimicrobial delivery systems for local infection prophylaxis in orthopedic- and trauma surgery. Biomaterials doi:https://doi.org/10.1016/i.biomaterials.2015.02.020 (2015).

12 Sorensen, T. S., Sorensen, A. I. \& Merser, S. Rapid release of gentamicin from collagen sponge. In vitro comparison with plastic beads. Acta orthopaedica Scandinavica 61, 353-356, doi:10.3109/17453679008993535 (1990).

13 Neut, D. et al. A biodegradable antibiotic delivery system based on poly(trimethylene carbonate) for the treatment of osteomyelitis. Acta Orthopaedica 80, 514-519, doi:10.3109/17453670903350040 (2009).

14 Boo, G.-J., Grijpma, D., Richards, R., Moriarty, T. \& Eglin, D. Preparation of gentamicin dioctyl sulfosuccinate loaded poly(trimethylene carbonate) matrices intended for the treatment of orthopaedic infections. Clinical bemorheology and microcirculation 60, doi:10.3233/CH-151935 (2015).

15 Tautzenberger, A., Kovtun, A. \& Ignatius, A. Nanoparticles and their potential for application in bone. Int J Nanomedicine 7, 4545-4557, doi:10.2147/ijn.S34127 (2012). 

coated poly(lactic-co-glycolic acid) microspheres. Journal of Controlled Release 127, 146-153, doi:https://doi.org/10.1016/j.jconrel.2008.01.017 (2008).

Raval, J. P., Naik, D. R., Amin, K. A. \& Patel, P. S. Controlled-release and antibacterial studies of doxycycline-loaded poly( $\varepsilon$-caprolactone) microspheres. Journal of Saudi Chemical Society 18, 566-573, doi:https://doi.org/10.1016/i.jscs.2011.11.004 (2014).

18 Rotman, S. G. et al. Development of bone seeker-functionalised microspheres as a targeted local antibiotic delivery system for bone infections. Journal of Orthopaedic Translation, doi:https://doi.org/10.1016/j.jot.2019.07.006 (2019).

Halasy-Nagy, J. M., Rodan, G. A. \& Reszka, A. A. Inhibition of bone resorption by alendronate and risedronate does not require osteoclast apoptosis. Bone 29, 553559, doi:https://doi.org/10.1016/S8756-3282(01)00615-9 (2001).

20 Paiva-Fonseca, F., Santos-Silva, A. R., Della-Coletta, R., Vargas, P. A. \& Lopes, M. A. Alendronate-associated osteonecrosis of the jaws: a review of the main topics. Med Oral Patol Oral Cir Bucal 19, e106-e111, doi:10.4317/medoral.19094 (2014).

21 Sekido, T. et al. Novel Drug Delivery System to Bone Using Acidic Oligopeptide: Pharmacokinetic Characteristics and Pharmacological Potential. Journal of Drug Targeting 9, 111-121, doi:10.3109/10611860108997922 (2001).

22 Ogawa, K. et al. Development of novel radiogallium-labeled bone imaging agents using oligo-aspartic acid peptides as carriers. PLoS One 8, e84335-e84335, doi:10.1371/journal.pone.0084335 (2013).

23 Jiang, T. et al. Poly aspartic acid peptide-linked PLGA based nanoscale particles: Potential for bone-targeting drug delivery applications. International Journal of Pharmaceutics 475, 547-557, doi:https://doi.org/10.1016/j.ipharm.2014.08.067 (2014).

24 Zhang, D. et al. Carboxyl-modified poly(vinyl alcohol)-crosslinked chitosan hydrogel films for potential wound dressing. Carbohydrate Polymers 125, 189-199, doi:https://doi.org/10.1016/j.carbpol.2015.02.034 (2015).

25 Sampath, S. S. \& Robinson, D. H. Comparison of new and existing spectrophotometric methods for the analysis of tobramycin and other aminoglycosides. Journal of pharmaceutical sciences 79, 428-431 (1990).

26 in Strategies to Modify the Drug Release from Pharmaceutical Systems (ed Marcos Luciano Bruschi) 63-86 (Woodhead Publishing, 2015).

27 Unagolla, J. M. \& Jayasuriya, A. C. Drug transport mechanisms and in vitro release kinetics of vancomycin encapsulated chitosan-alginate polyelectrolyte microparticles as a controlled drug delivery system. Eur J Pharm Sci 114, 199-209, doi:10.1016/j.ejps.2017.12.012 (2018).

28 Ritger, P. L. \& Peppas, N. A. A simple equation for description of solute release II. Fickian and anomalous release from swellable devices. Journal of Controlled Release 5, 37-42, doi:https://doi.org/10.1016/0168-3659(87)90035-6 (1987).

29 Wang, X. et al. In situ cross-linked and highly carboxylated poly(vinyl alcohol) nanofibrous membranes for efficient adsorption of proteins. Journal of Materials Chemistry B 3, 7281-7290, doi:10.1039/C5TB01192B (2015).

30 Giménez, V., Mantecón, A., Ronda, J. C. \& Cádiz, V. Poly(vinyl alcohol) modified with carboxylic acid anhydrides: crosslinking through carboxylic groups. Journal of Applied Polymer Science 65, 1643-1651, doi:10.1002/(SICI)10974628(19970822)65:8<1643::AID-APP20>3.0.CO;2-V (1997). 
31 Knaepler, H. Local application of gentamicin-containing collagen implant in the prophylaxis and treatment of surgical site infection in orthopaedic surgery. International Journal of Surgery 10, S15-S20, doi:https://doi.org/10.1016/j.ijsu.2012.05.020 (2012).

32 Kim, B.-N., Kim, E. S. \& Oh, M.-D. Oral antibiotic treatment of staphylococcal bone and joint infections in adults. Journal of Antimicrobial Chemotherapy 69, 309-322, doi:10.1093/jac/dkt374 (2013).

33 Chohan, Z. H., Supuran, C. T. \& Scozzafava, A. Metal binding and antibacterial activity of ciprofloxacin complexes. Journal of Ensyme Inbibition and Medicinal Chemistry 20, 303-307, doi:10.1080/14756360310001624948 (2005).

34 Nithya, R. \& Meenakshi Sundaram, N. Biodegradation and cytotoxicity of ciprofloxacin-loaded hydroxyapatite-polycaprolactone nanocomposite film for sustainable bone implants. International journal of nanomedicine 10 Suppl 1, 119-127, doi:10.2147/IJN.S79995 (2015).

35 Krause, K. M., Serio, A. W., Kane, T. R. \& Connolly, L. E. Aminoglycosides: An Overview. Cold Spring Harb Perspect Med 6, a027029, doi:10.1101/cshperspect.a027029 (2016).

36 Heeb, S. et al. Quinolones: from antibiotics to autoinducers. FEMS microbiology reviews 35, 247-274, doi:10.1111/j.1574-6976.2010.00247.x (2011).

37 Jeong, D., Joo, S.-W., Shinde, V. V., Cho, E. \& Jung, S. Carbohydrate-Based HostGuest Complexation of Hydrophobic Antibiotics for the Enhancement of Antibacterial Activity. Molecules 22, 1311, doi:10.3390/molecules22081311 (2017).

38 Maggi, N., Pasqualucci, C. R., Ballotta, R. \& Sensi, P. Rifampicin: A New Orally Active Rifamycin. Chemotherapy 11, 285-292, doi:10.1159/000220462 (1966).

39 Wang, Z., Wang, J., Zhang, M. \& Dang, L. Solubility of Erythromycin A Dihydrate in Different Pure Solvents and Acetone + Water Binary Mixtures between 293 K and $323 \mathrm{~K}$. Journal of Chemical \& Engineering Data 51, 1062-1065, doi:10.1021/je0505265 (2006).

40 Xie, S. et al. Design and synthesis of theranostic antibiotic nanodrugs that display enhanced antibacterial activity and luminescence. Proceedings of the National Academy of Sciences, 201708556, doi:10.1073/pnas.1708556114 (2017).

41 Shiina, I., Kubota, M., Oshiumi, H. \& Hashizume, M. An effective use of benzoic anhydride and its derivatives for the synthesis of carboxylic esters and lactones: a powerful and convenient mixed anhydride method promoted by basic catalysts. $J$ Org Chem 69, 1822-1830, doi:10.1021/jo030367x (2004).

42 Badal, S., Her, Y. F. \& Maher, L. J., 3rd. Nonantibiotic Effects of Fluoroquinolones in Mammalian Cells. J Biol Chem 290, 22287-22297, doi:10.1074/jbc.M115.671222 (2015). 


\section{Supplementary information and data}

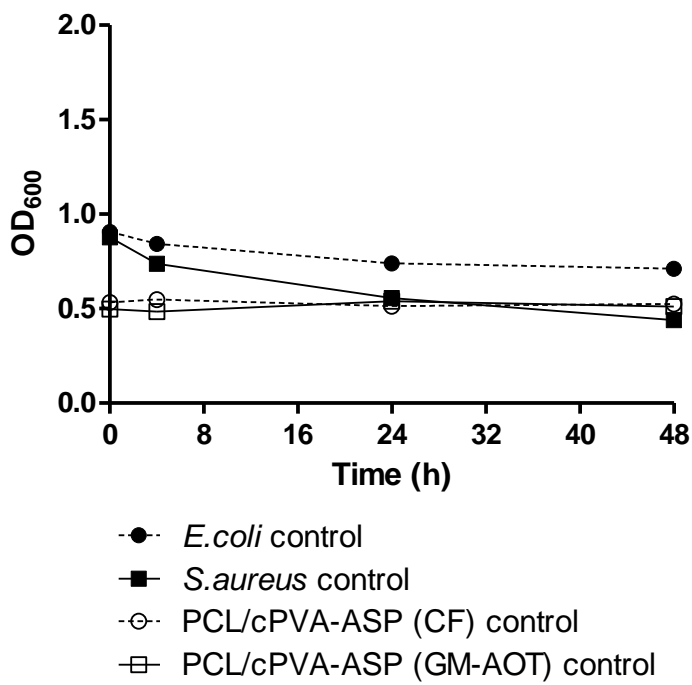

Figure 4.S1 - OD600 measurements of bacterial suspensions and antibiotic loaded PCL/cPVA-ASP microsphere dispersions over 48 hours.

OD600 of bacterial dispersions is slightly reduced over 48 hours in nutrientpoor PBS but is relatively stable for straightforward interpretation of antimicrobial effects of antibiotic loaded microsphere treatments. Dispersions of antibiotic loaded PCL/cPVA-ASP microspheres remain at a constant OD600 over the course of the experiment, simplifying interpretation of mixed dispersions of bacteria and microspheres in Figure 4.5 . 


\section{Chapter 5}

\section{Antibiotic loaded PCL-PEG nanoparticles}

S.G. Rotman ${ }^{1,2}$, D.W. Grijpma ${ }^{2}$, D. Eglin ${ }^{1,2}$ and O. Guillaume ${ }^{1}$

${ }^{1}$ AO Research Institute Davos, Davos Platz, Switzerland

${ }^{2}$ Department of Biomaterials Science and Technology, Faculty of Science and Technology, University of Twente, Enschede, The Netherlands 


\section{Introduction}

Achieving high local antibiotic concentrations during the treatment of bone infections can be a challenging prospect. The area of infected bone tissue or bone fractures have impaired vascularity leading to a reduced efficacy of systemic antibiotics. In order to improve treatment outcome for bone infection cases, antibiotic loaded biomaterials (ALB) must be locally introduced at the site of infection. Microspheres for bone targeted antibiotic delivery were fabricated in Chapter 3 and Chapter 4 of this thesis and showed enhanced delivery of antibiotics to mineralized substrates. While micronsized materials are generally easy to handle, fabricate and load with antibiotics, they might show limited tissue penetrating capacities. As bone targeting of particulate drug delivery systems is frequently achieved through surface functionalization, microspheres might be at a disadvantage compared to nanoparticles which possess a higher surface area per unit of mass.

This appendix reports on nanoparticle fabrication with poly ( $\epsilon$ caprolactone)-poly (ethylene glycol) (PCL-PEG) copolymers with Gentamicin-AOT (GM-AOT) load. In an approach similar to Yin et al.[1], carboxylic acid terminated PEG initiators were used to allow subsequent nanoparticle surface functionalization with the bone binding moiety alendronate (ALN). The size distribution of the nanoparticles is characterized, and GM-AOT release kinetics are reported.

\section{Materials and methods}

\section{Materials}

O-(2-Carboxyethyl) polyethylene glycol (PEG-COOH) $\left(\mathrm{Mw}=5,000 \mathrm{~g} \cdot \mathrm{mol}^{-}\right.$ $\left.{ }^{1}\right)$, poly(ethylene glycol) methyl ether (mPEG), alendronate sodium salt 
(ALN) and phosphate buffered saline (PBS) were purchased from Sigma
Aldrich
(Darmstadt,
Germany).
1-Ethyl-3-(3-

dimethylaminopropyl)carbodiimide (EDC) was purchased from Fluka (Buchs, Switzerland). Dichloromethane (DCM), diethyl ether, acetone, and dimethylformamide (DMF) solvents were bought from Carl Roth (Karlsruhe, Germany). All chemicals were used as delivered, unless stated otherwise.

\section{Synthesis of PCL-mPEG and PCL-PEG-COOH}

mPEG (5 gram, $1 \mathrm{mmol}$ ) and PEG-COOH (0.53 gram, $0.11 \mathrm{mmol})$ were separately dried under vacuum overnight in 3-neck flasks submerged in an oil bath at $70^{\circ} \mathrm{C}$. Molar quantities were determined according manufacturers labeling. $\epsilon$-caprolactone monomer was dehydrated by additions of calcium hydride and distilled under vacuum. 10 gram and 1 gram of $\epsilon$-caprolactone monomer was added to the mPEG and PEG-COOH, respectively. Stannous octoate $(0.02 \mathrm{mmol})$ was added as a catalyst. The ring-opening polymerization occurred at $130^{\circ} \mathrm{C}$ in inert argon environment for 3 days. Proton nuclear magnetic resonance $\left({ }^{1} \mathrm{H}-\mathrm{NMR}\right)$ measurements were performed to assess the presence of $\epsilon$-caprolactone monomers and to ensure complete polymerization. The PCL-mPEG and PCL-PEG-COOH copolymers were precipitated in ice-cold diethyl ether and the precipitate was dried under vacuum at $40^{\circ} \mathrm{C}$ until a steady weight was achieved after 3 days.

\section{Synthesis of PCL-PEG-ALN}

A $100 \mathrm{mg} / \mathrm{mL}$ solution of PCL-PEG-COOH in $10 \mathrm{~mL}$ acetone was made. EDC (50 mg, $0.32 \mathrm{mmol})$ and NHS (30 $\mathrm{mg}, 0.26 \mathrm{mmol})$ were added to the polymer solution and stirred overnight under ambient conditions. The 
carboxylic acid chain-end was converted into an active NHS-ester (PCLPEG-NHS). The product was precipitated in ice-cold diethyl ether and the precipitate was dried under vacuum for 4 hours. PCL-PEG-NHS was dissolved in $10 \mathrm{~mL}$ acetone and ALN (15 mg, $0.06 \mathrm{mmol}$ ) was dissolved in $10 \mathrm{~mL}$ DMSO. Both solutions were mixed and stirred for 24 hours under ambient conditions. The PCL-PEG-ALN polymers were precipitated in icecold diethyl ether and dried under vacuum for 4 hours. ${ }^{1} \mathrm{H}-\mathrm{NMR}$ samples consisted of dried PCL-PEG-ALN polymers dissolved in deuterated chloroform.

\section{Nanoparticle fabrication by nanoprecipitation}

PCL-mPEG (200 mg) and PCL-PEG-ALN (20 mg) was dissolved in $20 \mathrm{~mL}$ DMF. NP fabricated with a 1:10 ratio of ALN and MPEG terminated polymers will be specified as PLC-PEG-ALN NP. For antibiotic loaded NP, GM-AOT $(55 \mathrm{mg}$ ) was dissolved together with the polymers. The polymer solution was added dropwise to $400 \mathrm{~mL}$ MilliQ water under mild magnetic stirring (300 rpm). The NP dispersions were centrifuged at $3000 \mathrm{~g}$ for 10 minutes and lyophilized. Dried NP batches were stored at $4{ }^{\circ} \mathrm{C}$ in airtight containers.

\section{Antibiotic release experiment}

Lyophilized PCL-PEG-ALN NP were dispersed in $6 \mathrm{~mL}$ MilliQ $\mathrm{H}_{2} \mathrm{O}$. Triplicate samples were made by pipetting $2 \mathrm{~mL}$ of NP dispersion in dialysis tubes $(\mathrm{MW}$-cut off $=12-14 \mathrm{kDa})$ and the loaded and closed dialysis tubes were placed flacon tubes with $25 \mathrm{~mL}$ PBS as release buffer. Periodically, 1 $\mathrm{mL}$ of PBS aspirated and refreshed with fresh PBS. GM-AOT presence in the withdrawn PBS samples was assessed by amine-reactive O- 
phthaldialdehyde (OPA) assay, as is described elsewhere [2]. GM-AOT concentrations were calculated by a standard curve reference. The GM-AOT encapsulation efficiency (EE\%) was investigated by dissolving $10 \mathrm{mg}$ GMAOT loaded NP in tetrahydrofuran (THF) and quantifying the GM-AOT load by OPA assay.

$$
E E \%=\left(G M-A O T_{\text {Encapsulated }} / G M-A O T_{\text {Initial }}\right) \cdot 100 \%
$$

\section{Dynamic light scattering measurements}

The hydrodynamic diameter distribution of PCL-PEG-ALN NP was determined by dynamic light scattering (DLS) (Zetasizer, Malvern Panalytical) measurements. DLS samples were prepared taking a small volume from the NP dispersion directly after nanoprecipitation and by loading the dispersion into Zetasizer cuvettes avoiding the incorporation of bubbles. Particle sizes between $0.4 \mathrm{~nm}$ and $10 \mu \mathrm{m}$ could be detected during the measurement and all measurements were made in triplicate.

\section{Results and Discussion}

\section{${ }^{1} \mathrm{H}-\mathrm{NMR}$ characterization of synthesis products}

The ${ }^{1} \mathrm{H}-\mathrm{NMR}$ spectra of PCL-mPEG and PCL-PEG-COOH can be seen in Figure 5.1 and Figure 5.2, respectively. The polymer number-average molecular weight of the PEG and PCL segments of both copolymers were determined after peak integration of the shown spectra and can be found in Table 5.1. 


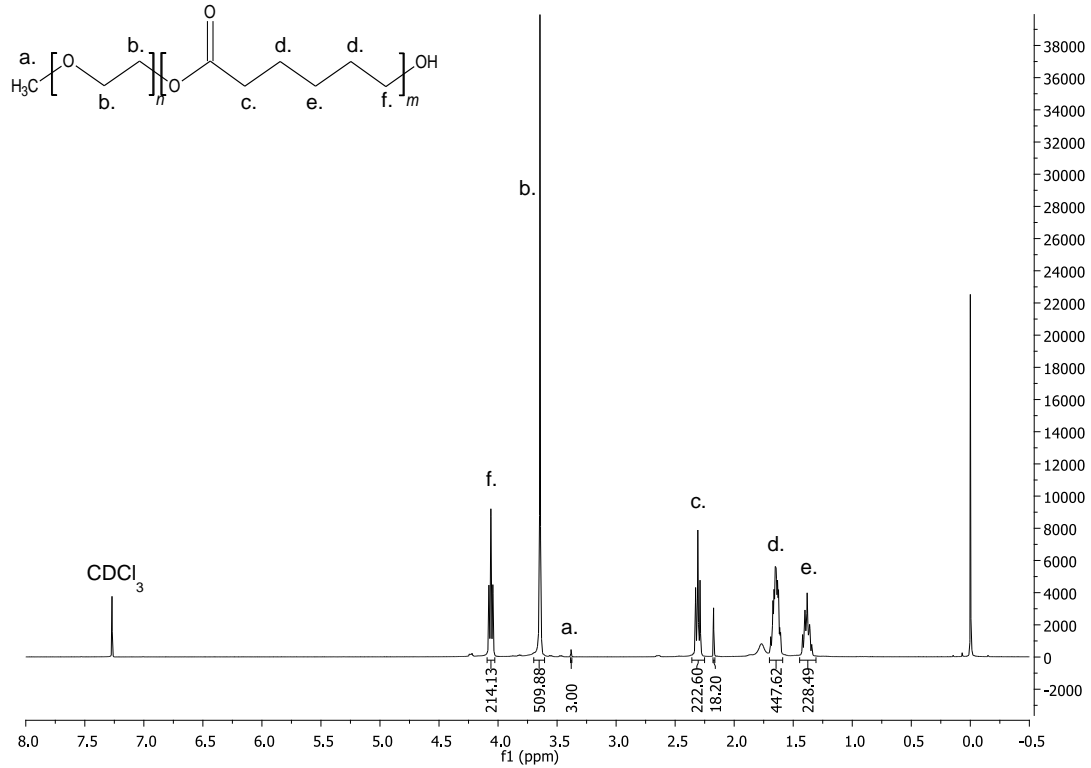

Figure 5.1 - ${ }^{1} \mathrm{H}-\mathrm{NMR}$ spectrum of PCL-mPEG. The three methyl protons were taken as a reference signal.

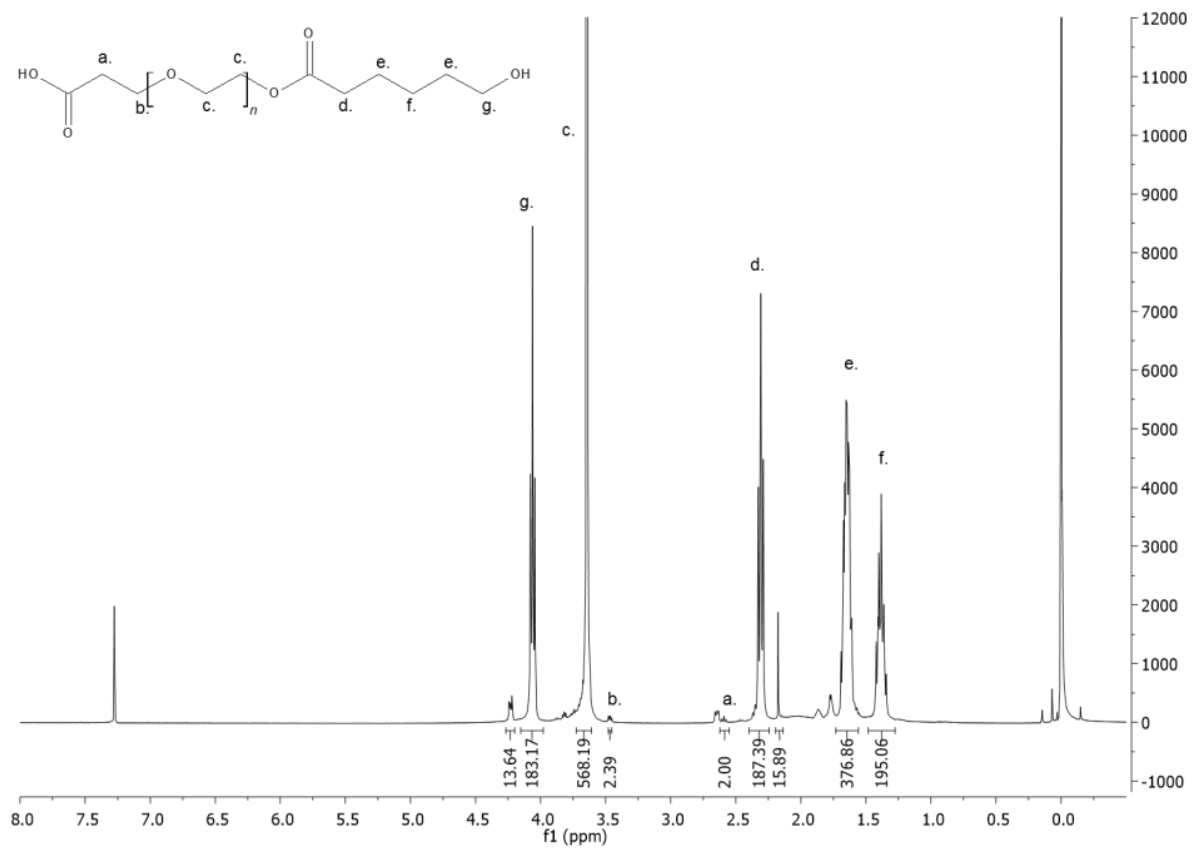

Figure 5.2 - ${ }^{1} \mathrm{H}-\mathrm{NMR}$ spectrum of PCL-PEG-COOH. The two protons adjacent to the carboxylic acid (labeled a.) are taken as a reference signal. 
Table 5.1 - Polymer number-average molecular weight $\left(\mathbf{M}_{n}\right)$ of the synthesized copolymers and their segments, as determined by ${ }^{1} \mathrm{H}-\mathrm{NMR}$ spectra of Figure 1 and 2.

\begin{tabular}{cccc}
\hline Copolymer & Segment & $\mathbf{M}_{\mathbf{n}}\left(\mathbf{g} \cdot \mathbf{m o l}^{-1}\right)$ & $\begin{array}{c}\text { copolymer } \mathbf{M}_{\mathbf{n}} \\
\left(\mathbf{g} \cdot \mathbf{m o l}^{-1}\right)\end{array}$ \\
\hline PCL-mPEG & PCL & 12.670 & 18.278 \\
& mPEG & 5.608 & \\
PCL-PEG-COOH & PCL & 11.132 & 17.382 \\
& PEG-COOH & 6.250 & \\
\hline
\end{tabular}

\section{ALN functionalization of PCL-PEG-COOH copolymers}

The ${ }^{1} \mathrm{H}-\mathrm{NMR}$ spectrum of PCL-PEG-ALN can be seen in Figure 5.3. Peaks of the PCL-PEG observed in Figure 5.2 are also identified in this spectrum. Theoretically, the three methylene $\left(\mathrm{CH}_{2}\right)$ groups of ALN have chemical shifts of 1.7 (a), 1.71 (b) and 3.16 (c) ppm. Due to the presence of broad methylene peaks of PCL at 1.4-1.8 ppm, only part of the ALN protons are observed in Figure 5.3. The ${ }^{1} \mathrm{H}$ NMR signal at $3.13-3.19 \mathrm{ppm}$ is attributed to the ALN protons $j$ (indicated by arrow) suggesting that a certain degree of ALN functionalization took place. Because of the low signal of the ALN protons compared to PCL-PEG protons, the degree of ALN functionalization was not calculated from the peak integration. 


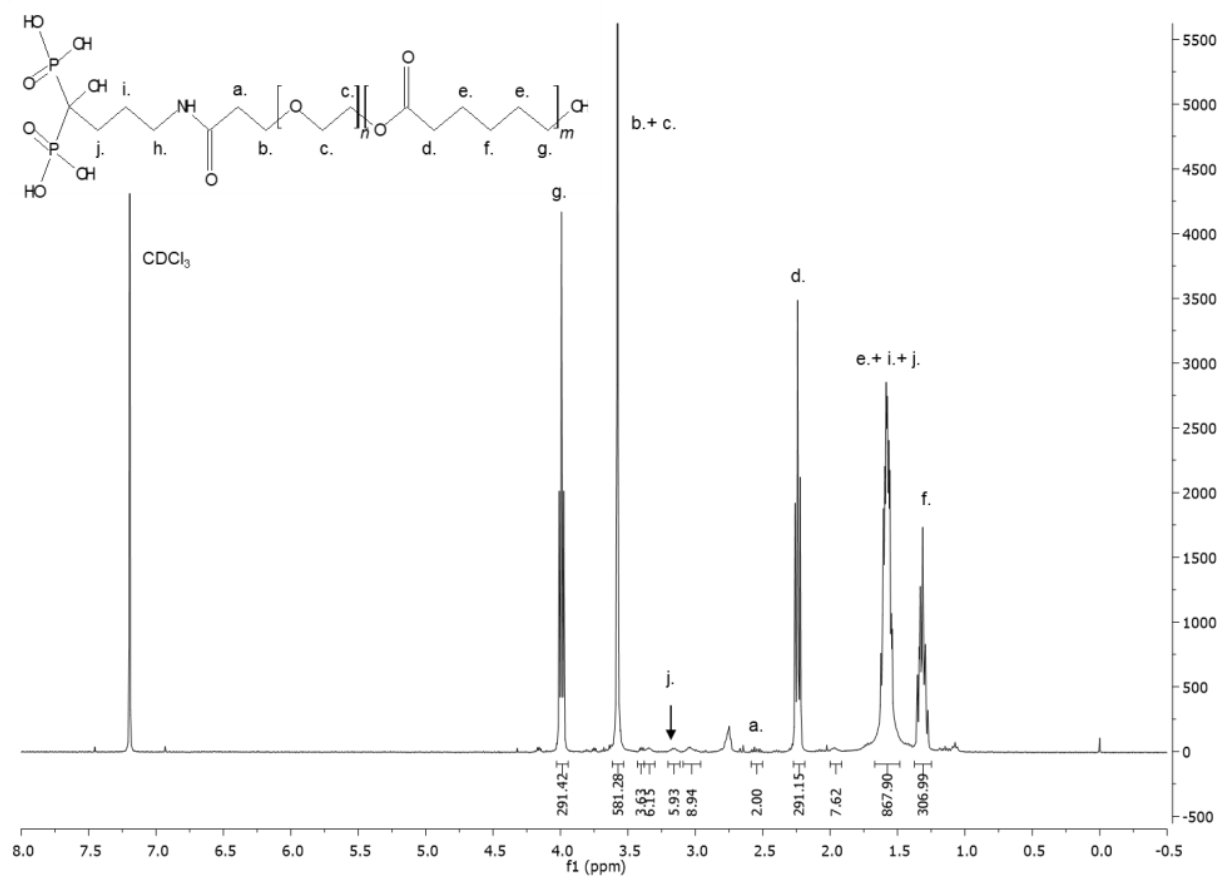

Figure $5.3-{ }^{1} \mathrm{H}-\mathrm{NMR}$ spectrum of PCL-PEG-ALN. The two protons labeled a. are taken as a reference signal. From all ALN protons, only $j$. does not overlap with other copolymer signals.

\section{GM-AOT release from PCL-PEG-ALN NP}

The release profile of GM-AOT from PCL-PEG-ALN can be seen in Figure 5.4. An initial burst release is observed for the first 24 hours and a plateau is reached after 48 hours. The dotted line represents the maximum drug concentration reached in the release media. The EE\% of GM-AOT calculated with eq. 1 is $70.1 \%$ and permitted to plot the drug percent release as a function of time (Figure 5.4B). The release profile plateaus at a near complete drug release of $92 \%$ of the drug load. Although a complete release of antibiotic is desirable over a retention of antibiotics in a slow degrading material such as PCL, the release kinetics are arguably too fast in order to 
have the prolonged therapeutic effect necessary for treatment of bone infection [3].

A

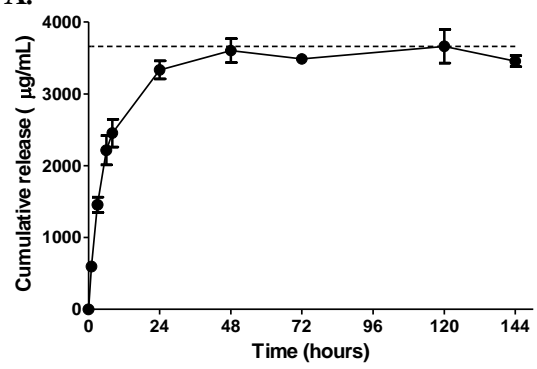

B.

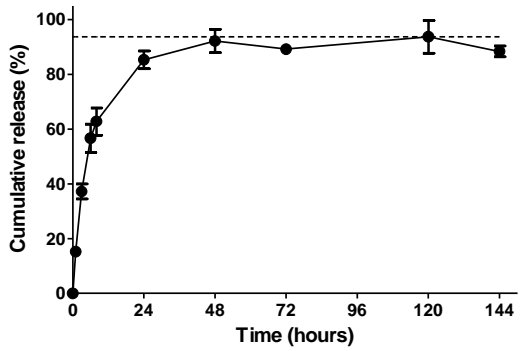

Figure 5.4 - A. Cumulative release profile of absolute quantities of GM-AOT from PCL-PEG-ALN NP. The dotted line indicates maximal release of GM-AOT at 3661 $\mu \mathrm{g} / \mathrm{mL}$ B. Cumulative release profile of GM-AOT relative to the initial drug load of the NP sample. The dotted line indicates maximal release of GM-AOT of $93 \%$ of initial drug load.

\section{DLS analysis of PCL-PEG-ALN NP}

The hydrodynamic diameter as measured by DLS are reported in Figure 5.5. An average hydrodynamic diameter value of $106 \mathrm{~nm}$ is measured with a minimum and maximum hydrodynamic diameter of 50 and $255 \mathrm{~nm}$, respectively. The NP size appears to be normally distributed.

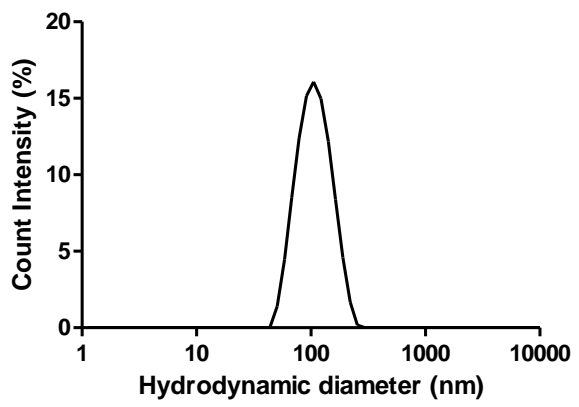

Figure 5.5 - The distribution of hydrodynamic diameters of PCL-PEG-ALN NP, as determined by an average of triplicate DLS measurements. 


\section{Conclusion}

In this work we investigated a PCL-PEG NP approach aimed at local antibiotic delivery to sites of bone infection. Even though GM-AOT loaded NP could be successfully fabricated by nanoprecipitation, the drug release profiles showed a high burst release in which over $92 \%$ of the drug load was released within 48 hours. The larger microsphere antibiotic delivery systems that were reported in Chapter 3 and 4, showed a more sustained release profile of GM-AOT over a period of 14 days. Because a longer antibiotic release is highly desired for bone infection treatments, the microsphere approach of Chapter 3 and 4 were considered the preferred strategies and were further developed.

\section{References}

1 Yin, Q. et al. Pamidronate functionalized nanoconjugates for targeted therapy of focal skeletal malignant osteolysis. Proceedings of the National Academy of Sciences, 201603316, doi:10.1073/pnas.1603316113 (2016).

2 Sampath, S. S. \& Robinson, D. H. Comparison of new and existing spectrophotometric methods for the analysis of tobramycin and other aminoglycosides. Journal of pharmaceutical sciences 79, 428-431 (1990).

3 Hatzenbuehler, J. \& Pulling, T. J. Diagnosis and management of osteomyelitis. American family physician 84, 1027-1033 (2011). 


\section{Chapter 6}

\section{Local bacteriophage delivery for treatment and prevention of bacterial infections}

S.G. Rotman ${ }^{1,2}$, E. Sumrall ${ }^{1}$, R. Ziadlou ${ }^{1,3}$, D.W. Grijpma ${ }^{2}$ R.G. Richards ${ }^{1}$, D. Eglin ${ }^{1,2}$ and T.F. Moriarty ${ }^{1}$

${ }^{1}$ AO Research Institute Davos, Davos Platz, Switzerland

${ }^{2}$ Department of Biomaterials Science and Technology, Faculty of Science and Technology, University of Twente, Enschede, The Netherlands

${ }^{3}$ Department of Biomedical Engineering, Faculty of Medicine, University of Basel, Basel, Switzerland 


\section{Abstract}

As viruses with high specificity for their bacterial hosts, bacteriophages (phages) are an attractive means to eradicate bacteria, and their potential has been recognized by a broad range of industries. Against a background of increasing rates of antibiotic resistance in pathogenic bacteria, bacteriophages have received much attention as a possible 'last-resort' strategy to treat infections. The use of bacteriophages in human patients is limited by their sensitivity to acidic $\mathrm{pH}$, enzymatic attack and short serum half-life. Loading phage within a biomaterial can shield the incorporated phage against many of these harmful environmental factors, and in addition, provide controlled release for prolonged therapeutic activity. In this review, we assess the different classes of biomaterials (i.e. biopolymers, synthetic polymers and ceramics) that have been used for phage delivery and describe the processing methodologies that are compatible with phage embedding or encapsulation. We also elaborate on the clinical or pre-clinical data generated using these materials. While a primary focus is placed on the application of phage-loaded materials for treatment of infection, we also include studies from other translatable fields such as food preservation and animal husbandry. Finally, we summarize trends in the literature and identify current barriers that currently prevent clinical application of phage-loaded biomaterials.

\section{Introduction to bacteriophage therapy}

Bacteriophages (phages) are natural viruses which harbor specificity for a bacterial host. After the phage infects the bacterial host by inserting its genetic material through the bacterial cell wall and membrane, the host's 
metabolism is rerouted to facilitate rapid phage replication. Towards the final stage of the bacteriophage replication cycle, phage-encoded endolysins disintegrate the bacterial cell wall from within, resulting in the release of the progeny phage. Phages are abundantly found in nature, including within the human body and contribute to homeostasis in the human microbiome.

Control of bacterial presence or numbers is necessary in many fields and disciplines, including food preservation [1], aquaculture [2], animal husbandry [3], plant preservation [4] and medicine, and this is routinely achieved by classical antimicrobial chemotherapy. However, with the rise of antibiotic resistance, alternatives to antibiotic therapy are becoming ever more necessary; phage therapy offers one possible alternative [5]. However, the application of phages in medicine could be challenging to implement on a broad scale, as it would need to conform with strict national and international regulations [6].

Over a century ago, Felix d'Hérelle successfully treated the first patient using phages [7], although such early phage therapy reports were questioned due to a lack of control groups and reproducibility [8]. Eventually, with the development of mass-produced antibiotics in the 1940 's, phages as an antimicrobial treatment were quickly forgotten in the western world. Phage research and treatments were, nevertheless, further developed in the former Soviet Union. Founded in 1923, the Eliava Institute in Georgia to this day continues to treat infected patients from all over the world using phages. In Wrocław, Poland, the Hirszfeld Institute has been founded in 1952 and remains the only specialized center for phage therapy in the European Union. In both Europe and the USA, 
recent clinical trials (as reviewed by Kutter et al. (2010) [9] and Furfaro et al. (2018) [6]) have marked the first step towards applying phages to treat antibiotic resistant microbial infections. The growing global interest in phage therapy is evident by the increasing number of recent clinical trials using oral, intravenous or topical phage.

However, there are several limitations to the utilization of bacteriophages in medicine. Where antibiotics often show a broad-spectrum of applicability, bacteriophages often show lytic activity on a single species or strain of bacteria. In a clinical setting, this would necessitate identification of the bacterial strain prior to the start of phage treatment, which might not be possible in the case of acute infections (e.g. sepsis). This narrow host-range is beneficial in the sense that it has less impact on nonpathogenic commensal bacteria, while for effective treatments bacteriophage cocktails can be prepared, which increases the host range of phages against bacteria at a site of infection. Another limitation of bacteriophage treatment is the duration of its activity in vivo. Much like pharmaceuticals that have therapeutic half-lives, bacteriophages also experience a decay in their lytic activity over time. The clearance of intravenously injected bacteriophage is complete within 60 minutes (T7 phage) and the half-life of $\lambda$-phage was shown to be approximately 6 hours [10]. Thus, to prevent in vivo bacteriophage decay, delivery strategies must be tailored to shield the phage from harmful environmental factors such as acidic/alkaline $\mathrm{pH}$ or serum inactivation. Sustained bacteriophage release from any biomaterial matrix locally implanted at the site of infection might prolong the effective treatment duration, resolving the necessity for repeated phage administration. This would be especially 
beneficial in the treatment of non-topical tissues such as bone where closure of the surgical site is highly desirable, and intravenous injection of phage is assumed to be ineffective.

In this review, we summarize the literature on the use of phage loaded biomaterials for enhanced stability and sustained release. While we maintain a primary focus on medical applications, we also include relevant literature on phage delivery in other fields (e.g. food industry), where appropriate. Finally, we offer what we consider promising advances in the field of infection control regarding combined phage treatments with antibiotics or the use of phage derived lysins.

\section{Advantages of bacteriophage loaded biomaterials for local delivery}

A major concern for the use of freely dispersed bacteriophage for medical treatments is their inactivation in physiological conditions. Firstly, exposure of bacteriophages to serum antibodies can result in immune clearance and reduction of phage infectivity [11]. Zaczek et al. has investigated the immune reaction of MS-1 phage on Anti-phage antibodies present in patients undergoing phage therapy. [12] An increased phage inactivation rate was observed due to increased anti-phage antibodies present in patient serum, although clinical outcome was minimally influenced even for patients with high expression of anti-phage antibodies. However, in different clinical settings where initial phage concentrations are lower or where phages have difficulty reaching the target tissue, the phage inactivation by the immune system could be an additional adverse effect resulting in a failed treatment outcome. 
Secondly, one of the harshest physiological conditions for bacteriophages is the acidic environment of the stomach, which limits efficient oral phage delivery to the gastrointestinal system. As was shown by Międzybrodzki et al., only a small fraction $(<0.1 \%)$ of oral administered phage was observed in the intestines, even after neutralizing antacid administration. The rationale behind several reviewed studies is to overcome such problems by protecting bacteriophages against harmful environments through embedding in a biomaterial matrix [13-17].

When repeated administration of freely dispersed phage is undesirable or impractical (e.g. for non-topical, deep-tissue/organ infections), implantation of phage-embedded biomaterials might lead to a sustained local release of the phage load. Without any means to propel itself, the embedded phage relies on diffusion to migrate from the biomaterial matrix in its search for a bacterial host [18]. The in vivo pharmacokinetics of bacteriophages differ greatly from antibiotics in term of tissue uptake and diffusion due to the fact that most antibiotics are (small) molecules while phages are an agglomeration of proteins [19]. This low mobility relative to antibiotics can be considered another benefit for local bacteriophage delivery, as phages would be released locally at the site of infection. Depending on the application, release kinetics of phages from a biomaterial matrix can be tuned by the biomaterial type and implant design (e.g. films, particles or hydrogels). Phage loading inside biomaterials (up to $10^{11} \mathrm{PFU} / \mathrm{mL}$ ) often exceeds the phage concentration used in recently proposed phage treatment protocols for non-topical infections $\left(10^{7}\right.$ $\mathrm{PFU} / \mathrm{mL}$, with rinsing volumes ranging from $10-40 \mathrm{~mL}$ [20,21]). Due to the high phage load of biomaterials, a sustained release of loaded phage is 
achievable and may be beneficial in order to maintain a prolonged therapeutic activity of released phage. Alternatively, in a prophylactic scenario a burst release of phage can be considered beneficial. Huff et al. (2002) added E. coli and SPR02 phage with two different multiplicities of infection (MOI), namely $\mathrm{MOI}=1\left(10^{4} \mathrm{CFU}\right.$ and $\left.10^{4} \mathrm{PFU}\right)$ and a MOI $=10,000\left(10^{4} \mathrm{CFU}\right.$ and $\left.10^{8} \mathrm{PFU}\right)$, and observed an increased survival rate of chickens from $65 \%$ at $\mathrm{MOI}=1$ to $100 \%$ at $\mathrm{MOI}=10,000$ after oral administration of phage and bacteria [22]. However, empirical evidence for a minimal effective local phage concentration for the treatment of local infections is not available and is most likely subject to phage-specific and patient-specific variability, making claims on 'ideal' phage release kinetics challenging at the present time.

Another benefit of loading phage inside a biomaterial is that it allows storage of phage in off-the-shelf formulations. Lyophilization is a common approach that can yield phage formulations that are often stable under ambient storage conditions [23]. Successful lyophilization of phage requires the addition of cryoprotectants to prevent the disintegration of the phage by the freezing process [23]. The preparation of lyophilized phage loaded biomaterials where the biomaterial itself acted as a protectant against freezing stresses has been described [24]. By freezedrying such a phage loaded construct, phage delivery systems with both long-term phage stability and storage capacity can be produced. 


\section{Considerations in manufacturing bacteriophage loaded biomaterials}

Due to the variety of bacteriophages described in the literature, and the widely recognized variability in their functionality under different conditions, it is difficult to make general statements on phage compatibility during incorporation with biomaterials. Thus, when incorporating bacteriophages on or in a biomaterial, the manufacturing procedure of the phage/biomaterial composite must be carefully considered and suited to the compatibility of the incorporated phage. Generally, three options are available for loading a biomaterial with bacteriophages: 1. embedding, 2. encapsulation and 3. surface functionalization through phage adsorption or covalent binding. Which strategy is used is generally dictated by the type of biomaterial and its processability. For example, ceramics such as hydroxyapatite (HAP) or other calcium phosphates (CAP) require such high sintering temperatures [25] that surface adsorption after sintering is the only viable method for direct phage loading for these materials. Other manufacturing processes such as organic solvent emulsions, electro-spinning/spraying or chemical modifications of biomaterials require conditions that could be harsh for the relatively fragile bacteriophages. The processing conditions that have been shown to be generally harmful to the viability of most phages were extremely acidic or alkaline environments [26], high temperatures [26], drying processes [27], ionic strength of solutions [28] and mechanical stresses [29]. Because varying observations have been made by different researchers when examining phage robustness, a freshly isolated bacteriophage should always be examined thoroughly in order to 
understand its limits before being subjected to processing with a biomaterial [30].

Strong alkaline or acidic environments have been recognized as detrimental for all types of bacteriophages, but a difference in tolerance has been reported between different phages. M13 and $\lambda$-phage, which are generally known as robust phages, can tolerate $\mathrm{pH}$ values between 3 and 11 for at least 20 minutes [31,32], but phages with a lower tolerance to extreme $\mathrm{pH}$ values have also been reported $[33,34]$. Several groups have compared effects of extreme $\mathrm{pH}$ conditions and serum exposure of multiple bacteriophages and noticed significant differences in lytic activity between the tested phages $[35,36]$. It remains relatively unknown which factors or characteristics allow for a phage to possess a degree of acid resistance. The acidic $\mathrm{pH}-\mathrm{level}$ of the stomach is a main concern for all orally applied phage formulations and can be addressed by embedding the phage in acid resistant polymer matrices [37].

The effect of high temperature on phage lytic activity has been studied extensively in order to understand the temperature range in which a phage can be processed and incorporated into a biomaterial. Seven species of bacteriophages were exposed to buffer at $60^{\circ} \mathrm{C}$ for 30 minutes, which led to titer reductions between 0.3 and $2.8 \log _{10}$ [38]. Heat inactivation occurs very rapidly at higher temperatures with a complete inactivation of lactococcal phages in reconstituted skim milk held for 5 minutes at $90^{\circ} \mathrm{C}$ [39]. Zottola and Marth (1966) concluded that it took a Streptococcus lactis phage 200 minutes to be inactivated at $70^{\circ} \mathrm{C}$, this only took 6 minutes at $77.8^{\circ} \mathrm{C}[40]$, signifying a rather precise temperature stability threshold. 
Due to the heat-sensitivity of phages, steam-sterilization (generally at $121^{\circ} \mathrm{C}$ for 20 minutes) of phage-loaded biomaterials is not a viable option. Other sterilization techniques such as ethylene oxide (EtO) gas sterilization and gamma $(\gamma)$ irradiation could potentially be applied to sterilize phage loaded biomaterials. Bienek et al. (2007) tested the effects of EtO gas sterilization and $\gamma$ irradiation on lyophilized $\Phi \mathrm{X}-174$ bacteriophage powders [41]. A 30-minute sterilization protocol with EtO gas resulted in a $5.3 \log _{10}$ reduction. Following a standard $\gamma$-irradiation sterilization protocol, a $3.8 \log _{10}$ titer reduction was observed. The same study investigated the effects of $\mathrm{EtO}$ and $\gamma$ sterilization when phage was inserted into cortical bone cavities which were sealed prior to the sterilization procedure, after which the phage was able to tolerate a 30 minute EtO exposure. These results could imply that embedded or encapsulated bacteriophage can be sterilized by short EtO treatments. However, the quantity of research on sterilization of encapsulated bacteriophages is very limited. Other techniques for sterilization of phage loaded biomaterials such as Ultraviolet (UV) irradiation or chemical sterilization with hydrogen peroxide or nitrogen dioxide remain unexplored. Alternatively, any material used in bacteriophage embedding must be sterilized prior to the embedding procedure, which can present other logistical challenges. Due to a lack of phage loaded biomaterials in clinics, practical considerations such as device sterilization are often overlooked. This critical gap in the literature needs to be successfully addressed in order to facilitate rapid clinical translation of phage delivery biomaterials. 
The presence of organic solvents and their volatility were also observed to be detrimental to phage activity [42]. Because of these practical limitations in applying organic solvents, water-soluble biopolymers such as sodium alginate are frequently used for bacteriophage encapsulation $[13,17,43]$. Agarwal et al. (2018) could also prevent exposure of phages to organic solvents by adding them on poly(lactide-co-glycolic acid) (PLGA) porous microspheres, which allowed the phage to be directly absorbed [44].

Dry formulations of embedded phage are known to be beneficial for long term storage, as they allow stability at room temperature, making storage in refrigerated conditions unnecessary [45]. Dry formulations of bacteriophages are also easily shipped and shared between labs, hospitals or potential industrial production plants without the need for wet or dry ice. The main techniques currently implemented for drying of bacteriophage formulations are ambient air drying [46], spray drying [47,48], lyophilization [24] or spray freeze-drying [49]. To protect bacteriophages from harmful drying effects during spray drying or lyophilization, (cryo-) protectants (i.e. skim milk, trehalose, sucrose) are added to the phage formulation. During lyophilization, cryoprotectants prevent damage to bacteriophages caused by the formation of ice crystals [50]. For spray drying, protectants shield the phage from thermal stresses and drying stresses [47]. The necessity for (cryo-)protectants in drying processes of phage loaded materials has been demonstrated in control groups of several studies [46,48]. Even upon addition of protectants, a 10 fold reduction in phage titer is typical after drying and long-term storage of phage [30] or phage loaded constructs [46]. Ma et al. (2008) embedded Staphylococcus aureus (S. aureus) phage $\mathrm{K}$ in alginate beads and proceeded to 
air-dry the beads that were soaked in solutions of protectants (i.e. skim milk, trehalose, sucrose) [51]. High PFU titers were maintained with a $0.7 \log _{10}$ reduction observed in the most favorable condition $(20 \%$ skim milk). While (cryo-)protectants can contribute to higher phage viability upon (freeze-)drying, reduced outcome of phage treatments when the applied phage products contain such (cryo-)protectants is a concern. Balogh et al. (2008) has reported that phage treatment in presence of skim milk failed to reduce bacterial spot disease severity on citrus fruits to the same extent as phage treatment without skim milk [52]. Therefore, the need to remove (cryo-)protectants from phage products must be considered on a phage-to-phage basis, but also depending on the (cryo)protectant used.

Besides environmental cues, some embedding processes can also be considered harmful to phages. Reduction of lytic activity by embedding phages in biomaterials is highly dependent upon the type of phage and biomaterial being used [29]. For example, Leung et al. (2019) showed that long-tailed phages were more susceptible to structural damage after jetnebulization compared to non-tailed phages [29]. Before any large-scale phage incorporation endeavor can be made, a microscopic or microbiological analysis of the embedded phage should be made to assess the degree of structural damage and ensure phage/methodology compatibility. For an extensive and in-depth review on the effect of environmental factors on phage stability we recommend the reader to a review paper by Malik et al. (2017) [53]. 


\section{Implementation of biomaterials for bacteriophage delivery}

\section{Organic materials}

\section{Biopolymers}

Biopolymers are polymeric macromolecules that can be produced by living organisms but can also be synthesized in industrial settings. Examples such as alginate, chitosan and fibrin are frequently used for bacteriophage embedding studies, due to their mild processing conditions and high capacity for molecular tailoring [54]. See Table 6.1 for a literature summary on this topic.

Alginate is a biocompatible polyanionic biopolymer and is widely used in food and pharmaceutical industries. On a molecular level, alginate consists of (1-4)-linked $\beta$-D-mannuronic acid (M) and $\alpha$-L-guluronic acid (G) units. These M- and G-units make up the alginate polymer in homopolymer M or $G$ sequences or in alternating MG blocks [55]. Alginate undergoes crosslinking (interaction of carboxylate groups on the G-units across two alginate chains) upon addition of divalent cations such as $\mathrm{Mg}^{2+}, \mathrm{Sr}^{2+}$ and $\mathrm{Ca}^{2+}$ [56]. Hence, alginates with a higher $\mathrm{G}$ content result in denser crosslinking and higher mechanical properties [57]. This characteristic can be utilized for tailoring release kinetics when alginate materials are used for drug- or phage-delivery applications. Alginate does not undergo enzymatic degradation in vivo as mammals lack the specific enzymes necessary [56]. Diffusion of divalent ions out of the gel results in structural disintegration of the alginate hydrogel. Anionic properties of alginate make for an excellent material for oral bacteriophage delivery due to the 
non-swelling properties of alginate in acidic environments like the stomach, protecting embedded phage from the harsh acidity [58]. The carboxylic acid groups of alginate are protonated in acidic environments, but at neutral $\mathrm{pH}$ levels (for example in the intestine), deprotonation of these carboxylic acids results in a great increase in swelling that allows for diffusion of drug or phages out of the alginate gels where it can be therapeutically active [59]. The size of the alginate construct seems to influence phage tolerance of acidic environments, as phage in millimetersized beads showed a $1 \log _{10}$ reduction [17] while phage embedded in microbeads with a diameter of $\pm 150 \mu$ m expressed $2-3 \log _{10}$ reduction in phage titer [60].

Chitosan is another biopolymer that has attracted attention in the field of bacteriophage delivery. Implementing shells of chitosan around phage loaded matrices like alginate can slow down alginate swelling and thus phage or $\mathrm{Ca}^{2+}$ diffusion out of the particle, resulting in a prolonged stability of the alginate matrix and a longer therapeutic activity of the bacteriophage delivery system [61]. From a chemical perspective, chitosan is a polysaccharide with repeating units of acetylated and non-acetylated forms of $\beta$-(1-4)-linked D-glucosamine. The solubility of chitosan is $\mathrm{pH}$ dependent as in acidic environments the protonation of its amine groups makes chitosan positively charged and water soluble. A multitude of studies have fabricated polyelectrolyte complexes of chitosan with anionic polymers such as alginate for drug delivery purposes [13,17,46]. Cationic materials have been recognized as having antiviral properties, with a 1 $\mathrm{mg} / \mathrm{mL}$ chitosan solution exerting a 2-log reduction of C2 and MS2 bacteriophages after coincubation for 1 minute [62]. These antiviral 
properties argue for the use of chitosan mainly as a shell structure around constructs with embedded phage as opposed to a matrix for direct phage embedding.

Release kinetics of bacteriophages from polysaccharide matrices is relatively quick, with reports stating a complete release of embedded phage ranging from minutes to approximately 12 hours in a neutral $\mathrm{pH}$ environment $[15,60]$. Often, the envisioned application for many of these studies is to embed phages to provide protection against the acidic $\mathrm{pH}$ in the stomach, followed by a quick release of phage in the intestine. This is achieved by entanglement of the hydrogel polymers upon protonation of carboxylic groups in acidic environments and subsequent loosening of the hydrogel polymers in neutral $\mathrm{pH}$ environments (e.g. the gut) [63]. For intestinal targets, phage loaded alginate and alginate-chitosan materials are highly suitable, as a fast release of phage and disintegration of the alginate matrix progresses while the alginate passes the intestines [64].

Recently, Rubalskii et al. (2019) used a commercially available fibrin glue

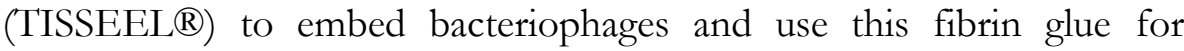
Psendomonas aeruginosa ( $P$. aeruginosa) phage PA01 delivery to treat local infections [65]. The fibrin matrix was completely dissolved after 11 days of incubation in saline solution but throughout this period, phages were consistently released at high titers of approximately $10^{9} \mathrm{PFU} / \mathrm{mL}$. The prolonged phage release provided by this fibrin material may make it suitable to treat local infections (e.g. orthopaedic or implant related infections). Further pre-clinical studies implementing TISSEEL $\mathbb{}$ adhesives for bacteriophage delivery would be of interest for rapid clinical translation of this established biomaterial. 
In one of the few published studies describing in vivo investigations of phage loaded biomaterials, Colom et al. (2017) showed the embedding of three different Salmonella phages in alginate $/ \mathrm{CaCO}_{3}^{-}$microbeads for intestinal delivery [60]. The relatively small size of the alginate $/ \mathrm{CaCO}_{3}$ beads allowed for complete intestinal release of embedded phage in 40 minutes. Embedded phage or free phage was orally administered 1 day prior to oral inoculation with S. typhimurium and continued daily for 8 days. When compared to control groups without treatment, a $1.7 \log _{10} \mathrm{CFU}$ reduction of $S$. typhimurium was observed for the embedded phage treatment, even after administration had ceased for 1 week. In the case of non-embedded bacteriophages, $S$. typhimurium $\mathrm{CFU}$ increased to the same level as the non-treated control group within 24 hours. The authors state that in all groups the $S$. typhimurium infection could not be completely prevented or eradicated due to high inoculation titers. Colom et al. states that an inoculation threshold that results in protection by phage released from alginate $/ \mathrm{CaCO}_{3}^{-}$should therefore be established so that the prophylactic effectiveness of the phage delivery system can be determined [60].

A recent clinical trial under the name PhagoBurn consisted of testing an alginate based wound dressing loaded with a cocktail of 12 P. aeruginosa phages and was considered the first clinical trial of phage therapy following good manufacturing and clinical practice guidelines [66]. In this clinical trial, commercially available alginate sheets were soaked in a phage solution to allow phage loading of the alginate construct. Subsequently, the alginate sheets were applied on P. aeruginosa infected burn wounds of randomized patients. This phage treatment was compared sulfadiazine 
silver cream treatment, a standard of care method consisting of a combination of sulfadiazine antibiotic and antimicrobial silver ions. The alginate wound dressings were able to reduce bacterial burden in the burn wounds, although no improved antimicrobial effects compared to the standard of care were observed. Due to these findings, the clinical trial was prematurely terminated, resulting in small number of recruited patients and low statistical power. However, the patients undergoing phage treatment did experienced less adverse events (e.g. septic shock, skin graft infection or impaired healing) compared the standard of care (23\% versus $54 \%$ respectively), which is an interesting finding that exposes potential antimicrobial or immunological benefits for phage therapy. Even though the PhagoBurn clinical trial did not show superior antimicrobial efficacy compared to available therapies, it did set a precedent in terms of regulatory procedures for future clinical trials with optimized bacteriophage delivery. 
Table 6.1 - Overview of biopolymers used for bacteriophage embedding. Abbreviations - CFU: Colony forming unit; nHAP: nano-hydroxyapatite; $\mathbf{O D}_{600}$ : Optical density at a wavelength of $600 \mathrm{~nm}$; PEI: Poly(ethylenimine); PFU: Plaque forming unit; SGF: Stimulated gastric fluid; SIF: Stimulated intestinal fluid.

\begin{tabular}{|c|c|c|c|c|c|c|c|}
\hline \multirow{2}{*}{$\begin{array}{l}\text { Envisioned } \\
\text { application }\end{array}$} & \multirow{2}{*}{$\begin{array}{l}\text { Biopolymers \& } \\
\text { construct }\end{array}$} & \multirow{2}{*}{$\begin{array}{l}\text { Embedded } \\
\text { phage }\end{array}$} & \multicolumn{4}{|c|}{ Relevant results } & \multirow[t]{2}{*}{ Ref. } \\
\hline & & & $\begin{array}{l}\text { Study } \\
\text { type }\end{array}$ & Phage release & Protective properties & Antimicrobial effects & \\
\hline $\begin{array}{l}\text { Intestinal phage } \\
\text { delivery }\end{array}$ & $\begin{array}{l}\text { Millimeter sized } \\
\text { beads with sodium } \\
\text { alginate and } \\
\text { chitosan core/shell } \\
\text { structure. Alginate } \\
\text { core viscosity was } \\
\text { increased by } \\
\text { supplementation } \\
\text { with honey/gelatin. }\end{array}$ & $\begin{array}{l}\text { E. coli phage } \\
\text { ZSEC5 }\end{array}$ & $\begin{array}{l}\text { In } \\
\text { vitro }\end{array}$ & $\begin{array}{l}\text { Complete phage } \\
\text { release in } 5 \text { hours } \\
\text { under SIF } \\
\text { conditions. }\end{array}$ & $\begin{array}{l}\text { Resisted acidic } \\
\text { environments } \\
(\mathrm{pH}=2) \text { for } 60 \\
\text { minutes and 3- } \\
\text { minute exposure } \\
\text { to } 80^{\circ} \mathrm{C} \text {, both with } \\
\text { a } 1 \log _{10} \text { reduction } \\
\text { of embedded } \\
\text { PFU. }\end{array}$ & $\begin{array}{l}\text { 4.5-5 } \log _{10} \text { reduction of E. coli } \\
\text { CFU after } 10 \text { hours } \\
\text { incubation. }\end{array}$ & $\begin{array}{l}\text { Abdelsatt } \\
\text { ar et al. } \\
2019 \\
{[17]}\end{array}$ \\
\hline $\begin{array}{l}\text { Intestinal phage } \\
\text { delivery }\end{array}$ & $\begin{array}{l}\text { Microspheres of } \\
\text { sodium alginate } \\
\text { and chitosan } \\
\text { core/shell structure. }\end{array}$ & $\begin{array}{l}\text { E. coli phage } \\
\text { O157:H7 }\end{array}$ & $\begin{array}{l}\text { In } \\
\text { vitro }\end{array}$ & $\begin{array}{l}65 \% \text { of embedded } \\
\text { phage released after } \\
6 \text { hours under SIF } \\
\text { conditions. }\end{array}$ & $\begin{array}{l}\text { Resisted acidic } \\
\text { environments } \\
\text { ( } \mathrm{pH}=2 \text { ) for } 60 \\
\text { minutes, with a } \\
1.5 \log _{10} \text { reduction } \\
\text { of embedded } \\
\text { PFU. }\end{array}$ & $\begin{array}{l}\text { After } 5 \text { hours incubation a } \\
2.5 \log _{10} \text { CFU reduction was } \\
\text { observed compared to } \\
\text { control cultures. }\end{array}$ & $\begin{array}{l}\text { Kim et al. } \\
2015 \\
{[13]}\end{array}$ \\
\hline $\begin{array}{l}\text { Intestinal phage } \\
\text { delivery }\end{array}$ & $\begin{array}{l}\text { Beads of Sodium } \\
\text { Alginate with either } \\
\text { a chitosan or a } \\
\text { polyethyleneimine } \\
\text { (PEI) coating }\end{array}$ & E. coliphage T4 & $\begin{array}{l}\text { In } \\
\text { vitro }\end{array}$ & $\begin{array}{l}\text { Release plateaus after } \\
12 \text { hours under SIF } \\
\text { conditions. Chitosan } \\
\text { and PEI coatings } \\
\text { delayed phage release } \\
\text { but reduced } \\
\text { cumulative release as } \\
\text { well. }\end{array}$ & $\begin{array}{l}\text { Polycationic } \\
\text { coatings increased } \\
\text { acid resistance of } \\
\text { embedded phage. } \\
4 \log _{10} \text { reduction in } \\
\text { Alginate beads vs. } \\
1 \log _{10} \text { reduction in } \\
\text { Alginate-PEI } \\
\text { beads. }\end{array}$ & Not assessed & $\begin{array}{l}\text { Moghtad } \\
\text { er et al. } \\
2016 \\
{[15]}\end{array}$ \\
\hline $\begin{array}{l}\text { Preservation of } \\
\text { meat }\end{array}$ & $\begin{array}{l}\text { Sodium Alginate } \\
\text { thin films }\end{array}$ & $\begin{array}{l}\text { P. fluorescens } \\
\text { phage } \phi I B B- \\
\text { PF7A }\end{array}$ & $\begin{array}{l}\text { In } \\
\text { vitro }\end{array}$ & $\begin{array}{l}\text { Complete release of } \\
\text { phage in } 3 \text { hours. }\end{array}$ & $\begin{array}{l}\text { PFU count was } \\
\text { reduced by } \\
4.2 \log _{10} \text { after } \\
\text { storage of } 12 \\
\text { weeks at } 4^{\circ} \mathrm{C} \text {. }\end{array}$ & $\begin{array}{l}P \text {. fluorescens } \text { CFU was } \\
\text { reduced by } 2 \log _{10} \text { compared } \\
\text { to control cultures. }\end{array}$ & $\begin{array}{l}\text { Alves et } \\
\text { al. } 2018 \\
{[43]}\end{array}$ \\
\hline $\begin{array}{l}\text { Phage delivery for } \\
\text { non-topical } \\
\text { infections }\end{array}$ & $\begin{array}{l}\text { Fibrin glue } \\
\text { (TISSEEL }(\text {, Baxter, } \\
\text { USA) }\end{array}$ & $\begin{array}{l}P . \text { aeruginosa } \\
\text { phage PA01 }\end{array}$ & $\begin{array}{l}\text { In } \\
\text { vitro }\end{array}$ & $\begin{array}{l}\text { Phage release was } \\
\text { followed for } 11 \text { days, } \\
\text { in which daily release } \\
\text { was between } 10^{9}-10^{10} \\
\text { PFU/day. }\end{array}$ & Not assessed & $\begin{array}{l}\text { Significant reductions in } \\
\mathrm{OD}_{600} \text { indicate antimicrobial } \\
\text { effects, }\end{array}$ & $\begin{array}{l}\text { Rubalskii } \\
\text { et al. } \\
2019 \\
{[65]}\end{array}$ \\
\hline $\begin{array}{l}\text { Treatment of } \\
\text { cystic fibrosis }\end{array}$ & $\begin{array}{l}\text { Spray dried or } \\
\text { lyophilized particles } \\
\text { of a mixture of } \\
\text { trehalose, mannitol } \\
\text { and leucine }\end{array}$ & $\begin{array}{l}\text { P. aeruginosa } \\
\text { phage PEV2 }\end{array}$ & $\begin{array}{l}\text { In } \\
\text { vitro }\end{array}$ & $\begin{array}{l}\text { Phage delivery was in } \\
\text { the order of } 10^{5} \mathrm{PFU} \\
\text { per dose from an } \\
\text { inhaler device. }\end{array}$ & Not assessed & Not assessed & $\begin{array}{l}\text { Leung } \\
\text { et al. } 2016 \\
{[49]}\end{array}$ \\
\hline $\begin{array}{l}\text { Intestinal phage } \\
\text { delivery }\end{array}$ & $\begin{array}{l}\text { Sodium alginate } \\
\text { and chitosan beads } \\
( \pm 800 \mu \mathrm{m} \text { diameter })\end{array}$ & $\begin{array}{l}\text { Salmonellla } \\
\text { phage Felix O1 }\end{array}$ & $\begin{array}{l}\text { In } \\
\text { vitro }\end{array}$ & $\begin{array}{l}\text { Near complete phage } \\
\text { release }\left(1.6 \cdot 10^{7} \mathrm{PFU}\right) \\
\text { in } 5 \text { hours under SIF } \\
\text { conditions. }\end{array}$ & $\begin{array}{l}\text { After } 60 \text { minutes } \\
\text { in } \mathrm{SGF}(\mathrm{pH}=2.4) \\
\text { a } 2.6 \log _{10} \\
\text { reduction in phage } \\
\text { titer was observed. } \\
\text { At } \mathrm{pH}=2 \text {, no titer } \\
\text { was observed after } \\
30 \text { minutes. }\end{array}$ & Not assessed & $\begin{array}{l}\mathrm{Ma} \\
\text { et al. } 2008 \\
{[46]}\end{array}$ \\
\hline
\end{tabular}


Continuation of Table 6.1

\begin{tabular}{|c|c|c|c|c|c|c|c|}
\hline $\begin{array}{l}\text { Intestinal phage } \\
\text { delivery }\end{array}$ & $\begin{array}{l}\text { Sodium } \\
\text { Alginate } / \mathrm{CaCO}_{3}{ }^{-} \\
\text {beads }( \pm 150 \mu \mathrm{m} \\
\text { diameter })\end{array}$ & $\begin{array}{l}\text { Salmonella } \\
\text { phages } \\
\text { UAB_Phi20/ } \\
\text { Phi78/ Phi87 }\end{array}$ & $\begin{array}{l}\text { In } \\
\text { vivo }\end{array}$ & $\begin{array}{l}80-100 \% \text { of all three } \\
\text { phages were released } \\
\text { after } 40 \text { minutes in } \\
\text { SIF. }\end{array}$ & $\begin{array}{l}\text { Titer loss after } 60 \\
\text { min exposure to } \\
\text { SGF } \\
\text { approximately } 2-3 \\
\log _{10} \text { for } \\
\text { UAB_Phi78 and } \\
\text { UAB_Phi87. No } \\
\text { significant titer } \\
\text { loss for } \\
\text { UAB_Phi20. }\end{array}$ & $\begin{array}{l}\text { Alginate } / \mathrm{CaCO}_{3} \text { - beads } \\
\text { showed } 1.7 \log _{10} \text { reduction } \\
\text { of excremental Salmonella. } \\
\text { Effect of free phage was } \\
\text { initially stronger but showed } \\
\text { no significant difference with } \\
\text { control group a week after } \\
\text { treatment ceased. }\end{array}$ & $\begin{array}{l}\text { Colom } \\
\text { et al. } \\
2017 \\
{[60]}\end{array}$ \\
\hline $\begin{array}{l}\text { Prophylactic } \\
\text { phage delivery in } \\
\text { an } \\
\text { osteointegrative } \\
\text { hydrogel }\end{array}$ & $\begin{array}{l}\text { Sodium } \\
\text { Alginate/nano- } \\
\text { hydroxyapatite } \\
\text { (nHAP) hydrogel }\end{array}$ & $\begin{array}{l}\text { E. faecalis } \\
\text { phages } \\
\text { vB_EfaS_LM99 }\end{array}$ & $\begin{array}{l}\text { In } \\
\text { vitro } \\
\& \text { ex } \\
\text { vivo }\end{array}$ & $\begin{array}{l}97 \% \text { of phage } \\
\text { released in } 24 \text { hours. } \\
\text { No difference in } \\
\text { phage release for } \\
\text { nHAP containing } \\
\text { hydrogels. }\end{array}$ & $\begin{array}{l}\text { Phage titers in the } \\
\text { hydrogel are stable } \\
\text { for } 7 \text { days }\end{array}$ & $\begin{array}{l}\text { Approximate } 2 \log _{10} \\
\text { reduction of CFU after } \\
\text { coincubation of hydrogels } \\
\text { with bacteria. On a chicken } \\
\text { femur ex vivo model a } 3 \log _{10} \\
\text { reduction of CFU was } \\
\text { observed after } 48 \text { hours. }\end{array}$ & $\begin{array}{l}\text { Barros } \\
\text { et al. } 2020 \\
{[67]}\end{array}$ \\
\hline $\begin{array}{l}\text { Phage delivery } \\
\text { triggered by } \\
\text { bacteria-secreted } \\
\text { hyaluronidase }\end{array}$ & $\begin{array}{l}\text { Composite films of } \\
\text { agarose and } \\
\text { hyaluronan }\end{array}$ & $\begin{array}{l}\text { S. aureus phage } \\
\phi K\end{array}$ & $\begin{array}{l}\text { In } \\
\text { vitro }\end{array}$ & $\begin{array}{l}\text { Phage release of } 10^{6} \\
\text { PFU/mL in } 4 \text { hours } \\
\text { of coincubation with } \\
\text { hyaluronidase }\end{array}$ & Not assessed & Not assessed & $\begin{array}{l}\text { Bean } \\
\text { et al. } 2014 \\
{[68]}\end{array}$ \\
\hline $\begin{array}{l}\text { Treatment of } \\
\text { osteomyelitis after } \\
\text { implant (i.e. } \\
\text { screw) removal }\end{array}$ & Alginate hydrogel & $\begin{array}{l}\text { CRISPR-Cas9 } \\
\text { modified phage } \\
\text { against } S \text {. aureus }\end{array}$ & $\begin{array}{l}\text { In } \\
\text { vivo }\end{array}$ & Not assessed & Not assessed & $\begin{array}{l}\text { No significant difference } \\
\text { between phage loaded } \\
\text { alginate and empty alginate } \\
\text { on CFU load of a bone } \\
\text { defect. CFU reduction } \\
\left(0.55 \log _{10}\right) \text { was observed in } \\
\text { the soft tissues surrounding } \\
\text { the bone defect. }\end{array}$ & $\begin{array}{l}\text { Cobb } \\
\text { et al, } \\
2019 \\
{[69]}\end{array}$ \\
\hline $\begin{array}{l}\text { Clinical trial: } \\
\text { treatment of burn } \\
\text { wound patients } \\
\text { with } P \text {. aeruginosa } \\
\text { monoinfections. } \\
\text { (Phagoburn } \\
\text { project) }\end{array}$ & $\begin{array}{l}\text { Alginate wound } \\
\text { dressing soaked in } \\
\text { phage cocktail } \\
\text { solution }\end{array}$ & $\begin{array}{l}\text { Phage cocktail } \\
\text { of } 12 P \text {. } \\
\text { aeruginosa } \\
\text { phages }\end{array}$ & $\begin{array}{l}\text { Clinic } \\
\text { al } \\
\text { trial }\end{array}$ & $\begin{array}{l}\text { Phage release was } \\
\text { assessed in vitro. } \\
\text { Burst release of } \\
\text { phages was } \\
\text { observed. Data set } \\
\text { was not published or } \\
\text { referenced. }\end{array}$ & Not assessed & $\begin{array}{l}\text { Phage loaded wound } \\
\text { dressing performed less } \\
\text { effectively as standard of } \\
\text { care control (sulfadiazine } \\
\text { silver cream). Less adverse } \\
\text { side effects were observed in } \\
\text { patients undergoing phage } \\
\text { treatment. }\end{array}$ & $\begin{array}{l}\text { Jault } \\
\text { et al. } 2019 \\
{[66]}\end{array}$ \\
\hline
\end{tabular}

\section{Synthetic polymers}

A synthetic polymer is a collective name for many different polymer classes where the polymer molecule is artificially synthesized in laboratory or industrial settings. Synthetic polymers (e.g. polyesters, polyethers, polyurethanes and polymethacrylates) make for attractive options as drug delivery systems due to the high control exerted over their physicochemical properties. Several of these synthetic polymers have been implemented for delivery of bacteriophages. In contrast to the usually water-soluble biopolymers, organic solvents might be necessary for processing of synthetic polymers. Solvent choice must be compatible with encapsulated or embedded phage to provide effective delivery. Literature 
on this topic is summarized in Table 6.2, with an emphasis on the manufacturing procedures of the phage delivery systems. Co-formulations of biopolymers and synthetic polymers are also listed in Table 6.2.

One of the most frequently applied synthetic polymers in biomedical technologies, including many drug delivery systems, is poly(ethylene glycol) (PEG) (also known as polyethylene oxide (PEO)). Compatibility of PEG with bacteriophages is evident, as it is a frequently used method for concentrating bacteriophage via precipitation in PEG solutions [7072]. As a hydrophilic polymer, PEG constructs can swell or dissolve in aqueous environments which can in turn trigger release of embedded bacteriophages [73]. Poloxamers are polymers whose structure and properties are very similar to PEG. Poloxamers consist of a hydrophobic polypropylene oxide (PPO) polymer with PEG chains on both chaintermini. Poloxamers show thermo-responsive behavior at elevated temperatures, and with the proper poloxamer composition (e.g. Poloxamer 407), undergo gelation at physiological temperatures. This is due to reduced solubility of the PPO segments upon temperature increase [74]. Poloxamer 407 has been investigated as a delivery material for bacteriophages, showing gelation of a 30\% Poloxamer 407 solution with phage dispersing in 2-3 minutes at physiological temperatures [75]. Poloxamer 407 hydrogels released bacteriophages over a 28-day period with daily released phage titers reducing from $10^{8} \mathrm{PFU}$ at day 1 to $10^{4} \mathrm{PFU}$ at day 28 [75]. With an in vitro phage delivery of approximately 1 month, such phage loaded hydrogel systems are a promising development for the treatment of infections where repeated phage administration is not viable or desirable (e.g. orthopaedic infections). 
The polyester poly(lactic-co-glycolic acid) (PLGA) is another synthetic polymer that has been used to embed or encapsulate bacteriophages $[24,44]$. Because of PLGA's limited water uptake and swelling properties, bacteriophage release from PLGA materials is much slower compared to hydrogel systems. Agarwal and coworkers have fabricated porous PLGA microparticles for bacteriophage delivery to treat lung infection in cystic fibrosis patients [44]. In order to have speedy and appropriate phage release kinetics from PLGA materials, the microparticles were additionally incubated in a buffered bacteriophage suspension to allow adsorption of phage into the microparticle pores and onto the surface. After inhalation of the PLGA microparticles, the local release of phage led to survival of mice that were administered a lethal inoculum of $P$. aeruginosa. Besides rapid release of phage from the microparticle, this approach has the additional advantage of preventing phage exposure to organic solvents that were used during PLGA microparticle fabrication.

Kaur and coworkers have implemented hydroxypropylmethlycellulose (HPMC) as a coating material for K-wires used in orthopaedic surgery $[76,77]$. HPMC is a synthetic polymer made from cellulose that is chemically modified by the addition of 2-hydroxypropylether groups on the polysaccharide chain. Combinations of phages and antibiotics were added to solutions of HPMC that were then used for repeated dip-coating of K-wires. In vitro, these coated K-wires loaded only with phages, released detectable quantities of phage for 4 days, resulting in a $3 \log _{10} \mathrm{CFU}$ reduction of multi-resistant $S$. aureus. When the material containing both phage and antibiotics was tested, an additive antimicrobial effect was seen [76]. In a pre-clinical mouse model of infection in the knee joint, these coated implants with phage or antibiotic load resulted in complete 
microbial clearance 15 days after implantation of an inoculated K-wire, while for combined phage and antibiotic coating complete microbial clearance was achieved after 10 days [77]. It must be mentioned that after 21 days, the untreated control group also showed an absence of CFU, which might limit the validity of this pre-clinical model.

Table 6.2 - Overview of the use of synthetic polymers for bacteriophage embedding. Abbreviations - CDA: cellulose diacetate; CFU: Colony-forming unit; DCM: Dichloromethane; HPMC: Hydroxypropylmethlycellulose; MRSA: Multiresistant Staphylococcus aureus; PEG: Poly(ethylene glycol); PEO: Poly(ethylene oxide); PFU: Plaque-forming unit; PLGA: Poly(lactic-co-glycolic acid); PVA: Poly(vinyl alcohol); RT: Room temperature.

\begin{tabular}{|c|c|c|c|c|c|c|c|}
\hline \multirow{2}{*}{$\begin{array}{l}\text { Envisioned } \\
\text { application }\end{array}$} & \multirow{2}{*}{$\begin{array}{l}\text { Polymer \& } \\
\text { construct }\end{array}$} & \multirow{2}{*}{$\begin{array}{l}\text { Embedded } \\
\text { phage }\end{array}$} & \multicolumn{4}{|c|}{ Relevant results } & \multirow[t]{2}{*}{ Ref. } \\
\hline & & & $\begin{array}{l}\text { Study } \\
\text { type }\end{array}$ & $\begin{array}{c}\text { Manufacturing \& Protective } \\
\text { properties }\end{array}$ & Phage release & Antimicrobial effects & \\
\hline $\begin{array}{l}\text { Protection of } \\
\text { phage against } \\
\text { acidic gastric } \\
\text { environment }\end{array}$ & $\begin{array}{l}\text { Microcapsules } \\
\text { of anionic } \\
\text { methacrylic } \\
\text { acid and } \\
\text { methyl } \\
\text { methacrylate } \\
\text { copolymer } \\
\text { (Eudragit }{ }^{\circ} \\
\text { S100) and } \\
\text { trehalose. }\end{array}$ & $\begin{array}{l}\text { S. enterica } \\
\text { phage Felix } \\
\text { O1 }\end{array}$ & In vitro & $\begin{array}{l}\text { Exposure to high } \\
\text { temperatures during } \\
\text { spray drying }\left(180^{\circ} \mathrm{C}\right) \\
\text { with low titer loss. } \\
\text { Copolymer:trehalose } \\
\text { (2:1) showed high phage } \\
\text { loading and acid } \\
\text { resistance. Dry storage } \\
\text { (RT, } 3 \text { months) caused } \\
\text { 1log }_{10} \text { titer reduction. }\end{array}$ & $\begin{array}{l}\text { Encapsulation of } \\
10^{9} \text { PFU/gram was } \\
\text { achieved. Presence } \\
\text { of trehalose } \\
\text { prevented a } 4 \log _{10} \\
\text { reduction observed } \\
\text { for copolymer } \\
\text { formulations. }\end{array}$ & Not assessed & $\begin{array}{l}\text { Vinner et } \\
\text { al. } \\
2019 \\
{[14]}\end{array}$ \\
\hline $\begin{array}{l}\text { Food } \\
\text { preservation } \\
\text { by } \\
\text { incorporation } \\
\text { in packaging } \\
\text { material }\end{array}$ & $\begin{array}{l}\text { Hollow fibers } \\
\text { mats of poly } \\
\text { (ethylene } \\
\text { oxide) (PEO) } \\
\text { and cellulose } \\
\text { diacetate } \\
\text { (CDA) blends. }\end{array}$ & $\begin{array}{l}\text { E. coli phage } \\
\text { T4 }\end{array}$ & In vitro & $\begin{array}{l}\text { PEO and CDA were } \\
\text { dissolved in } 98: 2 \mathrm{wt}^{0} \% \\
\text { chloroform/methanol. } \\
\text { Fiber mats fabricated by } \\
\text { coaxial electrospinning. } \\
\text { Fibers containing phage } \\
\text { had diameter range } 1.35 \text { - } \\
2.47 \mu \mathrm{m} \text {. }\end{array}$ & $\begin{array}{l}\text { Higher PEO } \mathrm{M}_{W} \\
\text { equals slower phage } \\
\text { release kinetics. } \\
\text { Increase of } \\
\text { hydrophobic CDA } \\
\text { slows fiber swelling } \\
\text { and thus phage } \\
\text { release kinetics. }\end{array}$ & Not assessed & $\begin{array}{l}\text { Korehei } \\
\text { et al. } \\
2014 \\
{[73]}\end{array}$ \\
\hline $\begin{array}{l}\text { Treatment of } \\
\text { bacterial lung } \\
\text { infections }\end{array}$ & $\begin{array}{l}\mathrm{W}_{1} / \mathrm{O} / \mathrm{W}_{2} \\
\text { Microparticles } \\
\text { of PLGA and } \\
\text { Pluronic } 92 \mathrm{~L} \text {, } \\
\text { fabricated by } \\
\mathrm{W} / \mathrm{O} / \mathrm{W} \\
\text { emulsions }\end{array}$ & $\begin{array}{l}\text { S. aureus } \\
\text { phage and } \\
\text { an isolated } \\
P . \text { aeruginosa } \\
\text { phage }\end{array}$ & In vitro & $\begin{array}{l}\mathrm{W}_{1}: 1 \% \text { aqueous solution } \\
\text { of Pluronic } 92 \mathrm{~L}, \mathrm{O}: 5 \% \\
\text { PLGA solution in DCM, } \\
\mathrm{W}_{2}: 1 \% \text { aqueous PVA } \\
\text { solution. Both } \mathrm{W}_{1} \text { and } \\
\mathrm{W}_{2} \text { contained phage. } \\
\text { Microparticles frozen in } \\
\text { liquid } \mathrm{N}_{2} \text { and lyophilized } \\
\text { without cryoprotectants. }\end{array}$ & $\begin{array}{l}\text { Phages in } \mathrm{W}_{1} \text { did } \\
\text { not efficiently } \\
\text { release. } \mathrm{W}_{1} / \mathrm{W}_{2} \\
\text { combined phage } \\
\text { release reached } 15 \% \\
\text { and } 25 \% \text { of } \\
\text { encapsulated } S \text {. } \\
\text { aureus and } P \text {. } \\
\text { aeruginosa phage } \\
\text { respectively. }\end{array}$ & $\begin{array}{l}\text { Lytic activity of both } S \text {. } \\
\text { aureus and } P \text {. aeruginosa } \\
\text { phage prevented bacterial } \\
\text { lawn formation after } 1 \text { - } \\
\text { or } 3 \text {-days storage at room } \\
\text { temperature. No PFU } \\
\text { observed after } 7 \text { days dry } \\
\text { storage at } 4^{\circ} \mathrm{C} \text { or RT. }\end{array}$ & $\begin{array}{l}\text { Puaperm } \\
\text { poonsiri } \\
\text { et al. } \\
2009 \\
{[24]}\end{array}$ \\
\hline $\begin{array}{l}\text { Treatment of } \\
\text { root canal } \\
\text { infections }\end{array}$ & $\begin{array}{l}\text { Poloxamer } \\
\text { P407 hydrogel } \\
(30 \% \mathrm{w} / \mathrm{v})\end{array}$ & $\begin{array}{l}\text { E. faecalis } \\
\text { phages } \\
\text { EFDG1 and } \\
\text { EFLK1 }\end{array}$ & In vivo & $\begin{array}{l}30 \% \text { poloxamer P407 } \\
\text { solution was made by } \\
\text { directly dissolving the } \\
\text { polymers in the phage } \\
\text { cocktail dispersion }\left(10^{9}\right. \\
\text { PFU } / \mathrm{mL}) \text {. }\end{array}$ & $\begin{array}{l}\text { Hydrogel gelation } \\
\text { occurred in } 2-3 \\
\text { minutes after } \\
\text { incubation at } 37^{\circ} \mathrm{C} \text {. } \\
\text { Released phage } \\
\text { gradually decreased } \\
\text { over } 28 \text { days (from } \\
10^{8} \mathrm{PFU} / \mathrm{mL} \text { to } 10^{4} \\
\mathrm{PFU} / \mathrm{mL} \text { ). }\end{array}$ & $\begin{array}{l}\text { In vitro CFU reduction of } \\
3 \text { - } 4 \log _{10} \text { reduction against } \\
\text { biofilm cultures. In vivo } \\
\text { efficacy (rat, 4-week old } \\
\text { root canal infection, } \\
2 \log _{10} \mathrm{CFU} \text { reduction) } \\
\text { and was superior to a } \\
\text { single } 10^{9} \mathrm{PFU} / \mathrm{mL} \\
\text { phage injection. }\end{array}$ & $\begin{array}{l}\text { Shlezinge } \\
\text { r et al. } \\
2019 \\
{[75]}\end{array}$ \\
\hline
\end{tabular}




\section{Continuation of Table 6.2}

\begin{tabular}{|c|c|c|c|c|c|c|c|}
\hline $\begin{array}{l}\text { Delivery of } \\
\text { phage to the } \\
\text { lungs of cystic } \\
\text { fibrosis } \\
\text { patients }\end{array}$ & $\begin{array}{l}\text { Hollow } \\
\text { microparticles } \\
\text { of poly } \\
\text { (lactic-co- } \\
\text { glycolic acid) } \\
\text { (PLGA) }\end{array}$ & $\begin{array}{l}\text { Multiple } P \text {. } \\
\text { aeruginosa } \\
\text { phages }\end{array}$ & In vivo & $\begin{array}{l}\text { Porous PLGA } \\
\text { microparticles with } \\
\text { phage loading after } \\
\text { particle formation. Phage } \\
\text { load of } 2.6 \cdot 10^{6} \mathrm{PFU} / \mathrm{mg} \\
\text { particle. Dry powder } \\
\text { formulation by } \\
\text { lyophilizing particles in } \\
\text { lactose solutions. }\end{array}$ & $\begin{array}{l}\text { Inhibitory zone of } \\
\text { P. aeruginosa around } \\
\text { phage loaded } \\
\text { PLGA particles was } \\
\text { microscopically } \\
\text { observed, indicating } \\
\text { phage release from } \\
\text { the PLGA } \\
\text { microparticle. }\end{array}$ & $\begin{array}{l}\text { In vitro biofilm CFU } \\
\text { reduction of } 2.5 \log _{10} \text {. } \\
\text { Mice inoculated with } P \text {. } \\
\text { aeruginosa were treated by } \\
\text { particle inhalation } \\
\text { resulting in a } 1.5 \log _{10} \\
\text { reduction of CFU } \\
\text { compared to free phage } \\
\text { administration. Survival } \\
\text { rate upon treatment was } \\
100 \% \text { versus } 13 \% \text { for } \\
\text { untreated control mice. }\end{array}$ & $\begin{array}{l}\text { Agarwal } \\
\text { et al. } \\
2018 \\
{[44]}\end{array}$ \\
\hline $\begin{array}{l}\text { Prophylactic } \\
\text { phage and } \\
\text { antibiotic } \\
\text { delivery on } \\
\text { orthopaedic } \\
\text { implants (K- } \\
\text { wires) }\end{array}$ & $\begin{array}{l}\text { Hydroxyprop } \\
\text { yl } \\
\text { methylcellulo } \\
\text { se (HPMC) } \\
\text { coating on K- } \\
\text { wires }\end{array}$ & $\begin{array}{l}\text { MRSA } \\
\text { phage MR-5 }\end{array}$ & In vitro & $\begin{array}{l}2 \% \text { and } 4 \% \text { HPMC } \\
\text { solutions containing } \\
\text { phage and/or linezolid } \\
\text { were used for dip coating } \\
\text { the K-wires. }\end{array}$ & $\begin{array}{l}\text { From } 2 \% \text { HPMC } \\
\text { coatings, release } \\
\text { was observed for } \\
48 \mathrm{~h} . \text { For } 4 \% \\
\text { HPMC coatings a } \\
\text { release period of } \\
96 \mathrm{~h} \text { was reached. }\end{array}$ & $\begin{array}{l}\text { Reduction of } 3 \log _{10} \text { of } \\
\text { CFU adhered to the K- } \\
\text { wire with HPMC-phage } \\
\text { coating. Additive effect } \\
\text { between linezolid and } \\
\text { phage was evident (> } \\
\text { 4log } \log _{10} \text { reduction of } \\
\text { adhered CFU). }\end{array}$ & $\begin{array}{l}\text { Kaur et } \\
\text { al. } \\
2014 \\
{[76]}\end{array}$ \\
\hline $\begin{array}{l}\text { Prophylactic } \\
\text { phage and } \\
\text { antibiotic } \\
\text { delivery on } \\
\text { orthopaedic } \\
\text { implants (K- } \\
\text { wires) }\end{array}$ & $\begin{array}{l}\text { Hydroxyprop } \\
\text { yl } \\
\text { methylcellulo } \\
\text { se (HPMC) } \\
\text { coating on K- } \\
\text { wires }\end{array}$ & $\begin{array}{l}\text { MRSA } \\
\text { phage MR-5 }\end{array}$ & In vivo & $\begin{array}{l}4 \% \text { HPMC solutions } \\
\text { containing phage and/or } \\
\text { linezolid were used for } \\
\text { dip coating the K-wires. }\end{array}$ & $\begin{array}{l}\text { In vivo release of } \\
\text { phage was not } \\
\text { tested. Outcome } \\
\text { based on CFU } \\
\text { reduction. }\end{array}$ & $\begin{array}{l}\text { Bacterial load diminished } \\
\text { fastest for } \\
\text { antibiotics/phage } \\
\text { combination. Phage, } \\
\text { antibiotic and combined } \\
\text { loaded HPMC coating } \\
\text { showed no CFU } \\
\text { observed in the soft } \\
\text { tissue }\end{array}$ & $\begin{array}{l}\text { Kaur et } \\
\text { al. } \\
2016 \\
{[77]}\end{array}$ \\
\hline $\begin{array}{l}\text { Treatment of } \\
\text { orthopaedic } \\
\text { infections }\end{array}$ & $\begin{array}{l}\text { Hydrogel of 4- } \\
\text { armed } \\
\text { polyethylene } \\
\text { glycol (PEG) } \\
\text { functionalized } \\
\text { with } \\
\text { crosslinking } \\
\text { moieties }\end{array}$ & $\begin{array}{l}P . \\
\text { aeruginosa } \\
\text { phages } \\
\text { ФPaer4/ } \\
\text { ФPaer } 14 . \\
\text { S. aureus } \\
\text { phage } \Phi \mathrm{K}\end{array}$ & In vivo & $\begin{array}{l}4 \text { arms of the PEG } \\
\text { macromers were } \\
\text { functionalized with } \\
\text { crosslinkers or tissue } \\
\text { adhesive peptides. }\end{array}$ & $\begin{array}{l}\text { Phage release } \\
\text { dictated by } \\
\text { enzymatic hydrogel } \\
\text { degradation. In } \\
\text { presence of } \\
\text { collagenase, } 50 \% \\
\text { phage release } \\
\text { observed in } 8 \\
\text { hours. }\end{array}$ & $\begin{array}{l}\text { In vitro anti-biofilm } \\
\text { experiments resulting in a } \\
1 \log _{10} \text { reduction in CFU. } \\
\text { In vivo, a } 0.5 \log _{10} \\
\text { reduction in CFU was } \\
\text { observed } 7 \text { days after } \\
\text { phage/ hydrogel } \\
\text { treatment. }\end{array}$ & $\begin{array}{l}\text { Wroe et } \\
\text { al. } \\
2020 \\
{[78]}\end{array}$ \\
\hline
\end{tabular}

\section{Liposomal encapsulation}

Liposomes are lipid bilayer spherical structures made from amphiphilic phospholipids. They present an interesting construct for intracellular bacteriophage delivery as the encapsulated bacteriophage is not embedded in a biomaterial matrix, but rather entrapped in a bilayer structure. Within a liposome, phages can be shielded from harmful environments while at the same time still being dispersed within the inner liposomal liquid. Intracellular delivery of bacteriophages could be achieved when the liposomal membrane fuses with eukaryotic cell membranes. It is currently debated if phages are able to diffuse through eukaryotic cell walls [79], making liposomes an attractive option for intracellular phage delivery. A 
very versatile selection of therapeutics can be loaded into liposomes as the aqueous core of liposomes is suitable for hydrophilic drugs or dispersed bacteriophages, while the bilayer shell can be loaded with hydrophobic drugs. Liposomes are compatible with many routes of administration, including parental, pulmonary and transdermal, while oral delivery requires specific additions (e.g. chitosan or PEG coatings) to the lipid bilayer to ensure the liposomes withstand acidic environments and bile salts of the gastrointestinal tract [80]. Liposomes can be fabricated by several techniques including lipid bilayer (thin film) rehydration or agitation/sonication of phospholipid solutions. For a complete overview of liposome preparation strategies, we recommend the work by Akbarzadeh et al. which reviews liposome production methods and potential applications [81]. Literature regarding liposomal phage carriers can be found in Table 6.3.

Gonzalez-Mendez et al. (2018) investigated the viability of encapsulating phages with liposomes and other carriers called niosomes and transferosomes [82]. Briefly, the membrane of niosomes consists of nonionic surfactants (e.g. Tween 20, Triton X-100, etc.) and the membrane of transferosomes is supplemented with edge activators that allow vesicle deformability of the vesicle, allowing for high tissue permeability. All three vesicle types could be loaded efficiently with bacteriophages $\left(10^{7}\right.$ $\mathrm{PFU} / \mathrm{mL}$ ), with encapsulation efficiencies ranging between $96.6 \%$ and 99.8\% [82]. Niosomes were found to protect best against low $\mathrm{pH}$ and high temperature environments, as it is believed that the non-ionic surfactants in niosomes are more stable in these conditions. Intracellular delivery of 
bacteriophages with the liposomes, niosomes or transferosomes, or any other antimicrobial functionality was not described in this work.

Cinquerrui et al. (2018) reported on S. aureus K-phage and E. coli T3 phage encapsulation in phospholipid DSCP:cholesterol liposomes prepared by microfluidic hydrodynamic flow focusing [83]. Increasing the cholesterol content of the lipid bilayer increased liposome size and broadened the liposomal size distribution. Significant differences were observed between the encapsulation efficiencies of K-phage and T3 phage with $\sim 10^{5}$ $\mathrm{PFU} / \mathrm{mL}$ and $\sim 10^{9} \mathrm{PFU} / \mathrm{mL}$ respectively. This discrepancy was attributed to a slow interaction between K-phage and the forming lipid bilayers, and to the phage size, with K-phage (and phage agglomerates) being too large to be effectively encapsulated. Interestingly, when testing encapsulation efficiency of phage in different initial titers, the highest titer $\left(\sim 10^{11}\right.$ $\mathrm{PFU} / \mathrm{mL}$ ) resulted in a lower quantity of phage as when a lower initial titer $\left(\sim 10^{10} \mathrm{PFU} / \mathrm{mL}\right)$ was attempted. This indicates that a high phage titer can cause agglomeration and thus hinder efficient encapsulation [83]. On a final note, Cinquerrui et al. (2018) also warns the reader about overestimation in published reports of phage encapsulation in liposomes [83]. They suggest this is due to externally adsorbed phages being included in the phage titer quantification. These phages would not benefit from any of the protective effects of liposomal entrapment and thus might not reach the target infection, resulting in lower efficient phage dosages than expected.

With the consensus that bacteriophages are unable or inefficient in diffusing past cellular membranes, liposomes form an interesting carrier material for intracellular bacteriophage delivery. However their efficacy in 
intracellular microbial clearance is not frequently reported, with only a hand-full of publications reporting successful intracellular phage delivery $[84,85]$. Endeavors in this field could yield valuable information regarding the efficacy of phage loaded liposomes against intracellular infections.

Table 6.3 - Overview of the use of liposomes for bacteriophage encapsulation. Abbreviations: CFU: Colony-forming units; HEPES: (4-(2-hydroxyethyl)-1piperazineethanesulfonic acid); PFU: Plaque-forming units.

\begin{tabular}{|c|c|c|c|c|c|c|c|}
\hline \multirow{2}{*}{$\begin{array}{l}\text { Envisioned } \\
\text { application }\end{array}$} & \multirow[t]{2}{*}{ Material \& construct } & \multirow{2}{*}{$\begin{array}{l}\text { Embedded } \\
\text { phage }\end{array}$} & \multicolumn{4}{|c|}{ Relevant results } & \multirow[t]{2}{*}{ Ref. } \\
\hline & & & $\begin{array}{l}\text { Study } \\
\text { type }\end{array}$ & $\begin{array}{c}\text { Manufacturing \& } \\
\text { encapsulation }\end{array}$ & $\begin{array}{c}\text { Phage release \& Protective } \\
\text { properties }\end{array}$ & Antimicrobial effects & \\
\hline $\begin{array}{l}\text { Food } \\
\text { processing } \\
\text { industry }\end{array}$ & $\begin{array}{l}\text { Niosomes, liposomes } \\
\text { and transfersomes from } \\
\text { commercial } \\
\text { phospholipid } \\
\text { formulations } \\
\text { (Pronanosome Lipo- } \\
\text { NTM and Pronanosome } \\
\text { Nio-NTM) }\end{array}$ & $\begin{array}{l}\text { Staphylococcal } \\
\text { phage phiIPLA- } \\
\text { RODI }\end{array}$ & $\begin{array}{l}\text { In } \\
\text { vitro }\end{array}$ & $\begin{array}{l}\text { For liposomes and } \\
\text { Niosomes the } \\
\text { formulations were } \\
\text { dissolved in } 0.05 \mathrm{M} \\
\text { HEPES buffer. The } \\
\text { solutions were } \\
\text { agitated by manual } \\
\text { shaking and } \\
\text { homogenization. } \\
\text { Transferosomes } \\
\text { were fabricated with } \\
\text { thin film hydration. }\end{array}$ & $\begin{array}{l}\text { Niosomes and } \\
\text { liposome showed 2- } \\
\log _{10} \text { reduction in PFU } \\
\text { after acid exposure. } \\
\text { Niosomes protected } \\
\text { the encapsulated phage } \\
\text { against elevated } \\
\text { temperatures of } 60^{\circ} \mathrm{C} \text {, } \\
\text { again showing a } 2-\log \\
\text { reduction where other } \\
\text { formulations expressed } \\
\text { no phage. }\end{array}$ & Not Assessed & $\begin{array}{l}\text { Gonzalez- } \\
\text { Menendez } \\
\text { et al. } \\
2018 \\
{[82]}\end{array}$ \\
\hline $\begin{array}{l}\text { Wound } \\
\text { dressing }\end{array}$ & $\begin{array}{l}\text { Uni-lamellar liposomes } \\
( \pm 200 \mathrm{~nm}) \text { : } \\
\text { - Phosphatidylcholi } \\
\text { ne } \\
\text { - Cholesterol } \\
\text { - Tween } 80 \\
\text { - Stearylamine } \\
\text { (weight ratio: } \\
7 / 3 / 1 / 0.5 \text { ) }\end{array}$ & $\begin{array}{l}\text { S. aureus phage } \\
\text { MR-5 and MR-10 }\end{array}$ & $\begin{array}{l}\text { In } \\
\text { vivo }\end{array}$ & $\begin{array}{l}\text { Encapsulation } \\
\text { efficiency of } 87 \% \text { for } \\
\text { both phages. }\end{array}$ & $\begin{array}{l}\text { Phage was quantifiable } \\
\text { for } 10 \text { days at the site } \\
\text { of the wound. } \\
\text { Liposome entrapped } \\
\text { phages initially showed } \\
\text { higher local PFU count } \\
\text { as free phage. }\end{array}$ & $\begin{array}{l}\text { Phage loaded } \\
\text { liposomes } \\
\text { performed } \\
\text { similarly as the } \\
\text { antibiotic control, } \\
\text { where free phage } \\
\text { was unable to } \\
\text { clear infection. } \\
\text { Wound healing } \\
\text { was faster for } \\
\text { liposome treated } \\
\text { group. }\end{array}$ & $\begin{array}{l}\text { Chhibber } \\
\text { et al. } \\
2018 \\
\text { [86] }\end{array}$ \\
\hline $\begin{array}{l}\text { Uptake of } \\
\text { encapsulated } \\
\text { phage by } \\
\text { intestinal cells }\end{array}$ & $\begin{array}{l}\text { Liposomes containing: } \\
\text { - phospholipids } \\
\text { - cholesteryl } \\
\text { - polyethylene } \\
\text { glycol } \\
600 \\
\text { - cholesterol } \\
\text { - cholesteryl3 } \beta \text { - } \\
\text { N(dimethylamino } \\
\text { ethyl)carbamatehy } \\
\text { drochloride (weight } \\
\text { ratio: } 1 / 0.1 / 0.2 / 0.7)\end{array}$ & $\begin{array}{l}\text { S. enterica phage } \\
\text { UAB_Phi20 }\end{array}$ & $\begin{array}{l}\text { In } \\
\text { vivo }\end{array}$ & $\begin{array}{l}10^{11} \mathrm{PFU} / \mathrm{mL} \\
\text { dispersed phage } \\
\text { could be } \\
\text { encapsulated though } \\
\text { lipid layer hydration } \\
\text { methods with an } \\
\text { efficacy of } 46 \% \text {. }\end{array}$ & $\begin{array}{l}\text { Upon oral } \\
\text { administration in mice, } \\
\text { phage labeled with } \\
\text { infrared marker was } \\
\text { detected in several } \\
\text { organs, including the } \\
\text { stomach, intestine, } \\
\text { spleen, liver and } \\
\text { muscle. }\end{array}$ & Not assessed & $\begin{array}{l}\text { Otero et al. } \\
2019 \\
{[16]}\end{array}$ \\
\hline $\begin{array}{l}\text { Phage delivery } \\
\text { for internal } \\
\text { medicine }\end{array}$ & $\begin{array}{l}\text { Liposomes containing: } \\
\text { - DSCP } \\
\text { phospholipids } \\
\text { - Cholesterol }\end{array}$ & $\begin{array}{l}\text { S. aureus phage K } \\
\text { ATCC 1985-B1 } \\
\text { and E. coli phage } \\
\text { ATCC 11303-B3 }\end{array}$ & $\begin{array}{l}\text { In } \\
\text { vitro }\end{array}$ & $\begin{array}{l}\text { Liposomes were } \\
\text { fabricated using } \\
\text { microfluidic } \\
\text { hydrodynamic flow } \\
\text { focusing, with a } \\
\text { phage load of } \sim 10^{8} \\
\text { PFU } / \mathrm{mL}\end{array}$ & $\begin{array}{l}\text { Encapsulation in } \\
\text { liposomes offered } \\
\text { limited protection } \\
\text { against acidic } \\
\text { environments, with a } \\
\text { 3log } \text { g }_{10} \text { reduction of } \\
\text { phage. }\end{array}$ & Not assessed & $\begin{array}{l}\text { Cinquerrui } \\
\text { et al. } \\
2018 \\
\text { [83] }\end{array}$ \\
\hline
\end{tabular}




\section{Continuation of Table 6.3}

\begin{tabular}{|c|c|c|c|c|c|c|c|}
\hline $\begin{array}{l}\text { Intestinal phage } \\
\text { delivery }\end{array}$ & $\begin{array}{l}\text { Liposomes }( \pm 300 \mathrm{~nm}) \\
\text { containing: } \\
\text { - 1,2-dilauroyl-rac- } \\
\text { glycero-3- } \\
\text { phosphocholine } \\
\text { (DOPC) } \\
\text { - Cholesteryl } \\
\text { - polyethylene } \\
\text { glycol 600 } \\
\text { sebacate } \\
\text { - cholesterol } \\
\text { - cholesteryl 33-N- } \\
\text { (dimethylaminoet } \\
\text { hyl) carbamate } \\
\text { hydrochloride } \\
\text { (molar ratio: } \\
\text { 1/0.1/0.2/0.7) }\end{array}$ & $\begin{array}{l}\text { Salmonella phages } \\
\text { UAB_Phi20/ } \\
\text { Phi78/ Phi87 }\end{array}$ & $\begin{array}{l}\text { In } \\
\text { vivo }\end{array}$ & $\begin{array}{l}\text { Liposomes formed } \\
\text { by thin-film } \\
\text { hydration method. } \\
\text { Encapsulation } \\
\text { efficiency between } \\
47-49 \% \text { for all three } \\
\text { phages. }\end{array}$ & $\begin{array}{l}\text { Liposomes provided } \\
\text { limited protection } \\
\text { against acidic } \\
\text { environments ( } 4-5 \log _{10} \\
\text { PFU titer reduction). }\end{array}$ & $\begin{array}{l}\text { Phage present in } \\
\text { the excrement of } \\
90 \% \text { of chickens } \\
\text { when liposome } \\
\text { encapsulated } \\
\text { phage was used. } \\
35 \% \text { for free } \\
\text { phage. After } 15 \\
\text { days, a } 2 \log ^{10} \\
\text { reduction in } \\
\text { Salmonella CFU } \\
\text { was observed in } \\
\text { the excrement of } \\
\text { chickens treated } \\
\text { with phage loaded } \\
\text { liposomes. }\end{array}$ & $\begin{array}{l}\text { Colom et } \\
\text { al. } \\
2015 \\
{[87]}\end{array}$ \\
\hline $\begin{array}{l}\text { Intracellular } \\
\text { phage delivery }\end{array}$ & $\begin{array}{l}\text { Liposomes }( \pm 100 \mathrm{~nm} \text { ) } \\
\text { - Phosphatidyl } \\
\text { choline } \\
\text { - Cholesterol } \\
\text { - Tween } \mathbf{8 0} \\
\text { - Stearylamine } \\
\text { (weight ratio: } \\
9 / 1 / 2 / 0.5 \text { ) }\end{array}$ & $\begin{array}{l}\text { K. pneumoniae } \\
\text { phage KPO1K2 } \\
\text { (MTCC 5831) }\end{array}$ & $\begin{array}{l}\text { In } \\
\text { vivo }\end{array}$ & $\begin{array}{l}\text { Thin film hydration } \\
\text { at } 40^{\circ} \mathrm{C} \text {. }\end{array}$ & $\begin{array}{l}\text { Liposomes provided } \\
\text { full protection against } \\
\text { phage neutralizing } \\
\text { antibodies and serum. } \\
\text { Liposome encapsulated } \\
\text { phage was observed in } \\
\text { the spleen of BALB/c } \\
\text { mice for } 14 \text { days, while } \\
\text { free phage was not } \\
\text { quantified after } 2 \text { days. }\end{array}$ & $\begin{array}{l}95 \% \text { intracellular } \\
\text { bacteria were } \\
\text { killed by liposome } \\
\text { encapsulated } \\
\text { phages, while only } \\
\text { a } 21 \% \text { reduction } \\
\text { was seen for free } \\
\text { phage. }\end{array}$ & $\begin{array}{l}\text { Singla et al. } \\
\text { 2016 } \\
\text { [85] }\end{array}$ \\
\hline $\begin{array}{l}\text { Intracellular } \\
\text { phage delivery }\end{array}$ & $\begin{array}{l}\text { Giant unilamellar } \\
\text { Liposomes ( } \mu \mathrm{m} \text { range) } \\
\text { - DOPC } \\
\text { - 1,2-dioleoyl-sn- } \\
\text { glycero-3- } \\
\text { phospho-L-serine } \\
\text { (weight ratio: } 50 / 50 \text { ) }\end{array}$ & $\begin{array}{l}\text { M. smegmatis phage } \\
\text { TM4 and E. coli } \\
\text { phage } \lambda \text { eyfp }\end{array}$ & $\begin{array}{l}\text { In } \\
\text { vitro }\end{array}$ & $\begin{array}{l}\text { Gel-assisted thin film } \\
\text { hydration and } \\
\text { inverse emulsion }\end{array}$ & $\begin{array}{l}\text { Only intracellular phage } \\
\text { presence was measured. } \\
4 \text {-fold increase of } \\
\text { intracellular phage was } \\
\text { observed by fluorescent } \\
\text { confocal microscopy. }\end{array}$ & Not assessed & $\begin{array}{l}\text { Nieth et al. } \\
2015 \\
{[84]}\end{array}$ \\
\hline
\end{tabular}

\section{Inorganic materials}

Examples of inorganic materials include ceramics, metals and salts. Although not as frequently investigated as organic phage carriers, delivery of bacteriophages using inorganic materials has received some attention, especially in the field of orthopaedic infections [25,88]. Literature regarding inorganic phage carriers can be found in Table 6.4. Ceramics like tricalcium phosphate (TCP) or hydroxyapatite (HAP) provide a scaffold for osteoconduction and ultimately for repair of bone defects [88] and the presence of bacteriophage could prevent the onset of infection at the defect site. Because of the high temperatures associated with ceramic sintering, phages must be adsorbed onto the construct after the fabrication process is complete. Meurice et al. (2012) prepared HAP and TCP pellets 
which were doped in a bacteriophage dispersion for 24 hours under refrigerated conditions [25]. An increase in porosity of the sintered pellets resulted in a delayed antimicrobial response, indicating a slower release of bacteriophage that were adsorbed on the inner porous structures of the pellets [25]. To show that the release of phages was not a mere initial burst release, the ceramic pellets were washed for 3 hours prior to antimicrobial testing. The ceramics with the most porosity showed the highest inhibitory properties after such a pre-wash was performed, corroborating the results that showed delayed release of phage from porous ceramic pellets [25].

Delivering phages intracellularly proves to be an additional challenge that must be overcome by designing appropriate delivery systems. Fulgione and coworkers used HAP nanoparticles ( $\pm 300 \mathrm{~nm}$ diameter) with adsorbed bacteriophage to achieve intracellular phage delivery [89]. Uptake of the HAP-phage complex in cancer cells was visualized by fluorescent microscopy of fluorescein isothiocyanate (FITC) labeled HAP. A $3 \log _{10}$ CFU reduction of intracellular Salmonella was observed for the HAP-phage complex while standalone phage caused no significant reduction of intracellular CFU [89]. As part of the pathology of bone infection, $S$. aureus bacteria are also known to reside intracellularly where antimicrobial treatments might be less effective [90]. While Salmonella osteomyelitis is relatively rare, this intracellular phage delivery system could present a promising tool to target intracellular $S$. aureus bacteria.

In the case of HAP materials, the adsorption kinetics of SR $\varphi 1$ phage appear to be driven by a difference in zeta potential (negative for most phages and slightly positive for biomimetic HAP), facilitating high phage adsorption in a time dependent manner [89]. With adsorption of phage 
being a surface interaction, porous materials show a much higher loading capacity than denser materials [25]. Intuitively, one could expect surface adsorbed phage release to proceed (e.g. detach) in a speedy fashion. Even though phage loaded HAP and TCP materials did show a large burst release, TCP pellets that were washed for up to 6 days could still establish an inhibition zone in an agar diffusion assay, regardless of their porosity [25].

Li et al. (2017) have attempted to improve biofilm penetration of bacteriophages by covalently binding E. coli and P. aeruginosa bacteriophage to chitosan coated magnetic iron oxide nanoparticles [91]. Upon application of a magnetic field, the phage-functionalized nanoparticles were able to be pushed through a soft-agar bacterial lawn in the direction of the magnetic field. As an unconventionally diluted agarose solution was used $(0.1 \%)$, it remains unclear if the in vitro model matches biofilms found in vivo and if nanoparticles would be able to penetrate such biofilms. Due to the small size of the delivery system $( \pm 100 \mathrm{~nm})$, cells are expected to internalize this phage delivery system through endocytosis potentially allowing for efficient intracellular phage delivery. However, the use of these functionalized nanoparticles for intracellular phage delivery, and whether the phage was able to replicate was not investigated. While deviating from the bacteriophage delivery approach in which phage must be released from its carrier material, covalent bacteriophage grafting onto surfaces can be also be considered a strategy to prevent bacterial colonization of surfaces or medical implants [92]. However, such surfaces would need a homogeneous and dense grafting of a broad selection of phages in order to achieve the desired anti-fouling effect. In the author's 
opinion, prophylactic phage release by one of the biomaterials reviewed in this work would be a more feasible strategy to prevent bacterial fouling of (medical) surfaces.

Table 6.4 - Overview of the use of non-polymeric materials for bacteriophage embedding. Abbreviations - CaP: Calcium phosphate; HA: Hydroxyapatite; OD600: Optical density at a wavelength of $600 \mathrm{~nm}$.

\begin{tabular}{|c|c|c|c|c|c|c|c|}
\hline \multirow{2}{*}{$\begin{array}{l}\text { Envisioned } \\
\text { application }\end{array}$} & \multirow{2}{*}{$\begin{array}{c}\text { Material \& } \\
\text { construct }\end{array}$} & \multirow{2}{*}{$\begin{array}{c}\text { Embedded } \\
\text { phage }\end{array}$} & \multicolumn{4}{|c|}{ Relevant results } & \multirow[t]{2}{*}{ Ref. } \\
\hline & & & $\begin{array}{l}\text { Study } \\
\text { type }\end{array}$ & Manufacturing process & $\begin{array}{c}\text { Phage release / } \\
\text { Protective properties }\end{array}$ & Antimicrobial effects & \\
\hline $\begin{array}{l}\text { Intercellular } \\
\text { delivery of } \\
\text { bacteriophages }\end{array}$ & $\begin{array}{l}\text { Biomimetic } \\
\text { HAP } \\
\text { nanocrystals }\end{array}$ & $\begin{array}{l}\text { S. rissen } \\
\text { phage SR } \\
\varphi 1\end{array}$ & $\begin{array}{l}\text { In } \\
\text { vitro }\end{array}$ & $\begin{array}{l}\text { Phage and HA } \\
\text { nanoparticles were } \\
\text { mixed together, and } \\
\text { phages adsorbed on } \\
\text { the HA material. } 10^{7} \\
\text { PFU/mg of HA } \\
\text { nanoparticles were } \\
\text { adsorbed after } 24 \\
\text { hours. }\end{array}$ & $\begin{array}{l}\text { Phage adsorbed } \\
\text { on HA } \\
\text { nanocrystals show } \\
\text { no reduction of } \\
\text { lytic activity after } \\
6 \text { weeks of storage } \\
\text { at } 4^{\circ} \mathrm{C} \text {. Adsorbed } \\
\text { phage shows no } \\
\text { inactivation due to } \\
\text { acidic } \\
\text { environment. }\end{array}$ & $\begin{array}{l}\text { A 3-log reduction of } \\
\text { S. rissen was } \\
\text { observed when } \\
\text { incubated with HA- } \\
\text { SR } \varphi 1 \text {. }\end{array}$ & $\begin{array}{l}\text { Fulgione } \\
\text { et al. } \\
2019 \\
{[89]}\end{array}$ \\
\hline $\begin{array}{l}\text { Delivery of } \\
\text { phage within } \\
\text { difficult to } \\
\text { penetrate } \\
\text { biofilms }\end{array}$ & $\begin{array}{l}\text { Chitosan } \\
\text { coated iron } \\
\text { oxide non- } \\
\text { colloidal } \\
\text { nanoparticles } \\
\text { for phage } \\
\text { grafting }\end{array}$ & $\begin{array}{l}\text { E. coli } \\
\text { phage } \\
\text { C3000 and } \\
\text { P. aeruginosa } \\
\text { phage } \\
\text { PA01 }\end{array}$ & $\begin{array}{l}\text { In } \\
\text { vitro }\end{array}$ & $\begin{array}{l}\text { Phage is conjugated } \\
\text { to the chitosan } \\
\text { coated iron oxide } \\
\text { nanoparticle though } \\
\text { carbodiimide } \\
\text { chemistry. }\end{array}$ & $\begin{array}{l}\text { No phage release, } \\
\text { particles possess } \\
\text { contact killing } \\
\text { though surface } \\
\text { functionalized } \\
\text { phages. }\end{array}$ & $\begin{array}{l}\text { Particles were able } \\
\text { to penetrate biofilms } \\
\text { better under } \\
\text { magnetic fields and } \\
\text { reduce the quantity } \\
\text { of biofilm with } 90 \% \\
\text { (compared to } 35 \% \\
\text { for free phage). }\end{array}$ & $\begin{array}{l}\text { Li et al. } 2017 \\
\text { [91] }\end{array}$ \\
\hline $\begin{array}{l}\text { Prophylactic } \\
\text { use in } \\
\text { orthopaedic } \\
\text { reconstruction } \\
\text { surgery }\end{array}$ & $\begin{array}{l}\text { Microporous } \\
\text { pelleted CaP } \\
\text { granules }\end{array}$ & $\begin{array}{l}\text { E. coli } \\
\text { phage } \lambda \text { vir }\end{array}$ & $\begin{array}{l}\text { In } \\
\text { vitro }\end{array}$ & $\begin{array}{l}\text { Phage was loaded by } \\
\text { allowing adsorption } \\
\text { at } 4^{\circ} \mathrm{C} \text { for } 24 \text { hours. }\end{array}$ & $\begin{array}{l}\text { the pellets were } \\
\text { washed for } 3 \\
\text { hours prior to } \\
\text { testing, resulting } \\
\text { in a 2-log } \\
\text { reduction of } \\
\text { phage load, } \\
\text { indicating strong } \\
\text { burst-like release } \\
\text { properties. } \\
\text { However, pellets } \\
\text { washed for } 6 \text { days } \\
\text { could still inhibit } \\
\text { bacterial growth in } \\
\text { a zone of } \\
\text { inhibition } \\
\text { experiment. }\end{array}$ & $\begin{array}{l}\text { Optical density } \\
\text { measurements show } \\
\text { a reduction when } \\
\text { phage loaded } \mathrm{CaP} \\
\text { materials were } \\
\text { introduced to the } \\
\text { culture. An increase } \\
\text { of porosity of the } \\
\text { sintered materials } \\
\text { results in higher } \\
\text { phage load and a } \\
\text { lower optical density } \\
\text { of the bacterial } \\
\text { culture. }\end{array}$ & $\begin{array}{l}\text { Meurice et al. } \\
2012 \\
{[25]}\end{array}$ \\
\hline $\begin{array}{l}\text { Prophylactic } \\
\text { use in } \\
\text { orthopaedic } \\
\text { reconstruction } \\
\text { surgery }\end{array}$ & $\begin{array}{l}\text { Macro porous } \\
\mathrm{CaCO}_{3} \text { and } \\
\text { HAP scaffold. }\end{array}$ & $\begin{array}{l}\text { E. coli } \\
\text { phage } \lambda \text { vir }\end{array}$ & \begin{tabular}{|l} 
In \\
vitro
\end{tabular} & $\begin{array}{l}\text { Phage was loaded by } \\
\text { allowing adsorption } \\
\text { at } 4^{\circ} \mathrm{C} \text { for } 24 \text { hours. }\end{array}$ & Not assessed & $\begin{array}{l}\mathrm{OD}_{600} \\
\text { measurements } \\
\text { indicate } \\
\text { antimicrobial effects } \\
\text { after } 90 \text { minutes of } \\
\text { coincubation. } \\
\text { Increased porosity }\end{array}$ & $\begin{array}{l}\text { Hornez et al. } 2013 \\
\text { [93] }\end{array}$ \\
\hline
\end{tabular}




\section{Combined delivery strategies with bacteriophages or bacteriophage derived products}

While many bacteriophage delivery systems have yet to undergo clinical trials, other strategies that implement combinations or elements of phage therapy are already being investigated. A few groups have experimented with delivery of phage derived lysins $[94,95]$ or combined delivery of phage and antibiotics $[69,77]$. As phages require a bacterial host, the effects of a second antimicrobial on phage amplification kinetics must be considered. Investigations on the dual treatments of antibiotics and bacteriophages solutions have been investigated in vitro by several groups [96,97]. Dickey $\&$ Perrot have investigated CFU reduction in biofilm experiments using 9 different antibiotics at 2x MIC and 10x MIC concentration together with S. aureus phage isolated from a Eliava PYO cocktail [97]. The phage-only treatment was able to reduce CFU in the biofilm by $1.3 \log _{10}$, and in this experimental setup could not eradicate the biofilm. For combined treatments they distinguished between simultaneous phage and antibiotic treatment or sequential treatment with phage followed by antibiotics. For simultaneous treatments, only enhanced bactericidal effects were seen for 2x MIC antibiotic concentrations, as adding phage to 10x MIC antibiotic treatment did not result in different outcomes and often severely reduced the presence of phage. For sequential phage and antibiotic treatments, results indicate that 7 out of 9 antibiotics at $2 \mathrm{x}$ MIC performed significantly better against biofilms. Without phage pre-treatment, only 1 antibiotic at $2 \mathrm{x}$ MIC showed antimicrobial effects against the $S$. aureus biofilms. These results show that the presence of phage conferred functionality of antibiotics at lower concentrations that would have been 
otherwise ineffective [97]. A combined delivery system that first releases bacteriophages, followed by a secondary release of a moderate quantity of antibiotics seems to be a promising prospect in the search for efficient anti-biofilm therapies.

Bacteriophage-encoded endolysins degrade the bacterial peptidoglycan from within at the end of the viral replication cycle. Since the peptidoglycan is heavily responsible for maintaining cellular integrity [98], any breach in its structure ultimately leads to cell lysis and release of phage from its host, making endolysins key to the use of bacteriophages as bactericidal agents. To take advantage of their important function, endolysins, and other similar bacteriolytic enzymes such as bacteriocins, can be produced as recombinant proteins and applied to bacteria from the outside as an antimicrobial, where they exert their effect in an identical manner. As Gram-positive bacteria lack an outer cell membrane, they are particularly susceptible to the effects of endolysins and can be eradicated extremely rapidly [99]. Unlike bacteriophages or most other antimicrobials, endolysins do not distinguish whether their target bacterium is metabolically active, making them ideal for targeting cells that would otherwise evade metabolically-dependent antimicrobial therapy [100]. Endolysins are known to recognize and cleave highly conserved residues within the bacterial peptidoglycan, presumably making it extremely difficult for bacteria to evolve resistance to their function [101]. Indeed, extensive efforts to generate endolysin resistant strains in the laboratory have proven unsuccessful, and resistance has never been observed [102-104]. 
The combination of hydrogels with antibacterial enzymes has proven useful due to the stabilizing effects that polycationic polymers have when the two components are combined in a complex [105]. However, this can have negative effects on the enzymatic activity [106], and has not been extensively explored. As with bacteriophages, hydrogels can be used for optimizing endolysin delivery to increase therapeutic efficacy, and a few studies have been performed using hydrogels to control their release. In one example, poly(N-Isopropylacrylamide) (PNIPAM) nanoparticles were successfully used in vitro to administer two bacteriolytic enzymes cystineand histidine-dependent aminohydrolase/peptidase (CHAPK) and lysostaphin against $S$. aureus via thermally triggered release at $37^{\circ} \mathrm{C}$ [95]. Also, lysostaphin has been successfully incorporated into a chitosan gel [107]. Encapsulation within such a hydrogel, while it does not necessarily form a complex, is thought to not only improve delivery, but to also enhance enzyme stability. This was proven in a recent prominent study which successfully used an injectable PEG-based hydrogel to deliver lysostaphin as a prophylactic against $S$. aureus orthopedic implant infection [94]. As a preventative measure, this method performed better than standard antibiotic therapy, and was improved upon in a later study which additionally incorporated bone morphogenetic protein-2 (BMP-2) into the same PEG-based hydrogel to promote healing of segmental bone defects [108]. It remains to be seen how the local delivery of bacteriolytic enzymes can be used to treat established infections, but as recent studies have demonstrated their effectiveness in eradicating biofilms [109-111], their outlook seems promising. 


\section{Limitations and critical gaps in the reviewed literature}

The antimicrobial potential of bacteriophages has been known for over a century, but large-scale clinical application of phages is still not implemented in most parts of the world. This originates from the fact that there are some critical gaps in our understanding of bacteriophage treatment and its effects on the patient and the patient's immune system, which complicates any endeavors to overcome key disadvantages through phage delivery with biomaterials. It is important to address that ideal bacteriophage concentrations and duration of phage treatment are not scientifically established. Without this knowledge it is challenging to design optimized phage delivering biomaterials with ideal phage release kinetics. More animal studies or even clinical trials studying these treatment parameters would give valuable insights that can set aims in terms of release kinetics for phage delivery studies with biomaterials.

Medical use of bacteriophages is not limited to monotherapies, and in vitro testing of combined therapy with antibiotic or phage-encoded endolysin delivery is a relatively recent development which requires further investigation. Open questions remaining for further investigation include assessing the immune response to administered bacteriophage loaded biomaterials and the potential adverse effects of these biomaterials on patients with impaired immune status. Combinational therapies with antiinflammatory agents would be another research focus which could potentially yield useful insights in suppressing adverse immune responses at the site of infection. 
Most reviewed studies assessed the release and functionality of biomaterials loaded with a limited number of different phages. Understandably from an academic standpoint this can be considered a good start when designing new biomaterials for phage delivery, but for clinical application, a phage loaded biomaterial should be able to release a wide variety of phages or even a cocktail of phages. Efficient delivery of a phage cocktail becomes even more important during treatment of acute infections or prophylaxis when the specific microbes are not previously identified. The wide range of architecture (e.g. tailed vs non-tailed) and size of bacteriophages makes variations in phage release kinetics from a biomaterial a possible concern. A big step towards clinical translation is made if biomaterials that show potential in phage delivery are further characterized for delivery of phage cocktails.

A practical limitation of bacteriophage loaded biomaterials is finding compatible sterilization techniques. The thermosensitive phages cannot undergo high temperature or steam sterilization and the few studies that focused on sterilization of phage loaded biomaterials showed that other common approaches such as EtO gas and $\gamma$-irradiation also reduced phage viability significantly [41]. Alternatively, the phage incorporation with biomaterials could occur under sterile conditions, but this invites other logistical difficulties and might go against clinical practices of re-sterilizing equipment prior to surgery. Other, more uncommon sterilization techniques such as UV and nitrogen dioxide remain to be investigated and should be pursued in order to find appropriate strategies for sterilization of phage loaded biomaterials. 


\section{Conclusions}

In conclusion, bacteriophage treatment represents a promising alternative to eradicate multidrug resistant bacteria. However, there are factors that limit their large-scale medical applicability, such as loss of activity in physiological conditions, which means they normally need to be repeatedly administered. As summarized in Tables $1-4$, large $\log _{10}$ reductions of bacterial burden were observed when incorporation of phage in a biomaterial matrix could establish a highly concentrated local release of phages in proximity to the bacteria. Treatment of different infections requires the phage to be incorporated in a variety of biomaterial constructs. Phage delivery to the gut requires an acid resistant material, like alginate, for efficient phage deposition, while phage adsorbed to nanomaterial could enable the phage to infect intracellular bacteria. Embedding, encapsulating or adsorbing bacteriophages in or onto carrier materials can potentially expose the phage to adverse conditions, to which the response can be very phage specific. Thorough compatibility testing and characterization is therefore required before any phage be loaded into a biomaterial. With the wide range of available biomaterials, and a vast number of differently behaving bacteriophages, much experimental work must be performed to optimize the bacteriophage delivery and patient outcome. Nevertheless, against a background of bacterial antibiotic resistance, new and exciting phage delivery systems are expected to make their appearance in clinical trials and hopefully in the clinics in order to alleviate infection burden in global healthcare. 


\section{Acknowledgements}

This work was published as part of the JPIAMR: Cross-border research project ANTI-BIOLAB (Grant number: 40AR40_185917).

\section{References}

1 Founou, L. L., Founou, R. C. \& Essack, S. Y. Antibiotic Resistance in the Food Chain: A Developing Country-Perspective. Front Microbiol 7, 1881-1881, doi:10.3389/fmicb.2016.01881 (2016).

2 Culot, A., Grosset, N. \& Gautier, M. Overcoming the challenges of phage therapy for industrial aquaculture: A review. Aquaculture $\mathbf{5 1 3}$ doi:10.1016/j.aquaculture.2019.734423 (2019).

3 Cuong, N. V., Padungtod, P., Thwaites, G. \& Carrique-Mas, J. J. Antimicrobial Usage in Animal Production: A Review of the Literature with a Focus on Lowand Middle-Income Countries. Antibiotics (Basel) 7, 75, doi:10.3390/antibiotics7030075 (2018).

4 McManus, P. S., Stockwell, V. O., Sundin, G. W. \& Jones, A. L. Antibiotic use in plant agriculture. Annual review of phytopathology 40, 443-465, doi:10.1146/annurev.phyto.40.120301.093927 (2002).

5 Ventola, C. L. The antibiotic resistance crisis: part 1: causes and threats. P T 40, 277-283 (2015).

6 Furfaro, L. L., Payne, M. S. \& Chang, B. J. Bacteriophage Therapy: Clinical Trials and Regulatory Hurdles. Front Cell Infect Microbiol 8, 376-376, doi:10.3389/fcimb.2018.00376 (2018).

$7 \quad$ Sulakvelidze, A. \& Kutter, E. (2004).

8 Wittebole, X., De Roock, S. \& Opal, S. M. A historical overview of bacteriophage therapy as an alternative to antibiotics for the treatment of bacterial pathogens. Virulence 5, 226-235, doi:10.4161/viru.25991 (2014).

9 Kutter, E. et al. Phage therapy in clinical practice: treatment of human infections. Current pharmaceutical biotechnology 11, 69-86, doi:10.2174/138920110790725401 (2010).

10 Prasuhn, D. E., Jr. et al. Plasma clearance of bacteriophage Qbeta particles as a function of surface charge. $J$ Am Chem Soc 130, 1328-1334, doi:10.1021/ja075937f (2008).

11 Prasuhn, D. E. et al. Plasma Clearance of Bacteriophage Q $\beta$ Particles as a Function of Surface Charge. $J$ Am Chem Soc 130, 1328-1334, doi:10.1021/ja075937f (2008).

12 Żaczek, M. et al. Antibody Production in Response to Staphylococcal MS-1 Phage Cocktail in Patients Undergoing Phage Therapy. Front Microbiol 7, 1681 1681, doi:10.3389/fmicb.2016.01681 (2016).

13 Kim, S., Jo, A. \& Ahn, J. Application of chitosan-alginate microspheres for the sustained release of bacteriophage in simulated gastrointestinal conditions. 
International Journal of Food Science \& Technology 50, 913-918, doi:10.1111/ijfs.12736 (2015).

Vinner, K. G. et al. Microencapsulation of Salmonella-Specific Bacteriophage Felix O1 Using Spray-Drying in a pH-Responsive Formulation and Direct Compression Tableting of Powders into a Solid Oral Dosage Form. Pharmaceuticals 12, doi:10.3390/ph12010043 (2019).

15 Moghtader, F., Eğri, S. \& Piskin, E. Phages in modified alginate beads. Artificial Cells, Nanomedicine, and Biotechnology 45, 357-363, doi:10.3109/21691401.2016.1153485 (2017).

16 Otero, J. et al. Biodistribution of Liposome-Encapsulated Bacteriophages and Their Transcytosis During Oral Phage Therapy. Front Microbiol 10, doi:10.3389/fmicb.2019.00689 (2019).

17 Abdelsattar, A. S., Abdelrahman, F., Dawoud, A., Connerton, I. F. \& ElShibiny, A. Encapsulation of E. coli phage ZCEC5 in chitosan-alginate beads as a delivery system in phage therapy. AMB Express 9, doi:10.1186/s13568-0190810-9 (2019).

18 Barr, J. J. et al. Subdiffusive motion of bacteriophage in mucosal surfaces increases the frequency of bacterial encounters. Proceedings of the National Academy of Sciences of the United States of America 112, 13675-13680, doi:10.1073/pnas.1508355112 (2015).

19 Nilsson, A. S. Pharmacological limitations of phage therapy. Upsala Journal of Medical Sciences 124, 218-227, doi:10.1080/03009734.2019.1688433 (2019).

20 Onsea, J. et al. Bacteriophage Application for Difficult-to-treat Musculoskeletal Infections: Development of a Standardized Multidisciplinary Treatment Protocol. Viruses 11, 891, doi:10.3390/v11100891 (2019).

21 Chan, B. K. et al. Phage treatment of an aortic graft infected with Pseudomonas aeruginosa. Evol Med Public Health 2018, 60-66, doi:10.1093/emph/eoy005 (2018).

22 Huff, W. E. et al. Prevention of Escherichia coli respiratory infection in broiler chickens with bacteriophage (SPR02). Poultry science 81, 437-441, doi:10.1093/ps/81.4.437 (2002).

23 Puapermpoonsiri, U., Ford, S. J. \& van der Walle, C. F. Stabilization of bacteriophage during freeze drying. International journal of pharmaceutics 389, 168 175, doi:10.1016/j.ijpharm.2010.01.034 (2010).

24 Puapermpoonsiri, U., Spencer, J. \& van der Walle, C. F. A freeze-dried formulation of bacteriophage encapsulated in biodegradable microspheres. European journal of pharmaceutics and biopharmaceutics : official journal of Arbeitsgemeinschaft fur Pharmazentische Verfahrenstechnik e.V 72, 26-33, doi:10.1016/j.ejpb.2008.12.001 (2009).

25 Meurice, E. et al. New antibacterial microporous CaP materials loaded with phages for prophylactic treatment in bone surgery. Journal of Materials Science: Materials in Medicine 23, 2445-2452, doi:10.1007/s10856-012-4711-6 (2012).

26 Jonczyk, E., Klak, M., Miedzybrodzki, R. \& Gorski, A. The influence of external factors on bacteriophages--review. Folia microbiologica 56, 191-200, doi:10.1007/s12223-011-0039-8 (2011).

27 Clark, W. A. Comparison of several methods for preserving bacteriophages. Appl Microbiol 10, 466-471 (1962). 
28 Jensen, H. B. \& Kleppe, K. Effect of ionic strength, $\mathrm{pH}$, amines and divalent cations on the lytic activity of T4 lysozyme. European journal of biochemistry 28, 116-122, doi:10.1111/j.1432-1033.1972.tb01891.x (1972).

29 Leung, S. S. Y. et al. Jet nebulization of bacteriophages with different tail morphologies - Structural effects. International journal of pharmaceutics 554, 322 326, doi:https://doi.org/10.1016/j.ijpharm.2018.11.026 (2019).

30 Ackermann, H.-W., Tremblay, D. \& Moineau, S. Long-term bacteriophage preservation. WFCC Newsletter 38, 35-40 (2004).

31 Branston, S. D., Stanley, E. C., Ward, J. M. \& Keshavarz-Moore, E. Determination of the survival of bacteriophage M13 from chemical and physical challenges to assist in its sustainable bioprocessing. Biotechnology and Bioprocess Engineering 18, 560-566, doi:10.1007/s12257-012-0776-9 (2013).

Jepson, C. D. \& March, J. B. Bacteriophage lambda is a highly stable DNA vaccine delivery vehicle. Vaccine 22, 2413-2419, doi:10.1016/j.vaccine.2003.11.065 (2004).

33 Kerby, G. P. et al. Purification, pH Stability and Sedimentation Properties of the T\&lt;sub\&gt; $\& 1$; / sub\&gt; Bacteriophage of Escherichia Coli. The Journal of Immunology 63, 93 (1949).

34 Khawaja, K., Abbas, Z. \& Rehman, S. Isolation and characterization of lytic phagesTSE1-3 against Enterobacter cloacae. Open Life sciences. Open Life Sciences 11, 287-292, doi:10.1515/biol-2016-0038 (2016).

35 Knezevic, P. et al. Phages of Pseudomonas aeruginosa: response to environmental factors and in vitro ability to inhibit bacterial growth and biofilm formation. Journal of Applied Microbiology 111, 245-254, doi:10.1111/j.13652672.2011.05043.x (2011).

36 O'Flynn, G., Coffey, A., Fitzgerald, G. F. \& Ross, R. P. The newly isolated lytic bacteriophages st104a and st104b are highly virulent against Salmonella enterica. Journal of Applied Microbiology 101, 251-259, doi:10.1111/j.13652672.2005.02792.x (2006).

37 Zelasko, S., Gorski, A. \& Dabrowska, K. Delivering phage therapy per os: benefits and barriers. Expert Review of Anti-infective Therapy 15, 167-179, doi:10.1080/14787210.2017.1265447 (2017).

38 Mocé-Llivina, L., Muniesa, M., Pimenta-Vale, H., Lucena, F. \& Jofre, J. Survival of bacterial indicator species and bacteriophages after thermal treatment of sludge and sewage. Appl Environ Microbiol 69, 1452-1456, doi:10.1128/aem.69.3.1452-1456.2003 (2003).

39 Murphy, J., Mahony, J., Bonestroo, M., Nauta, A. \& van Sinderen, D. Impact of thermal and biocidal treatments on lactococcal 936-type phages. International Dairy Journal 34, 56-61, doi:https://doi.org/10.1016/i.idairyj.2013.06.011 (2014).

40 Zottola, E. A. \& Marth, E. H. Thermal Inactivation of Bacteriophages Active Against Lactic Streptococci. Journal of Dairy Science 49, 1338-1342, doi:https://doi.org/10.3168/jds.S0022-0302(66)88091-8 (1966).

41 Bienek, C. et al. Development of a bacteriophage model system to investigate virus inactivation methods used in the treatment of bone allografts. Cell and Tissue Banking 8, 115-124, doi:10.1007/s10561-006-9018-8 (2007). 
42 Salalha, W., Kuhn, J., Dror, Y. \& Zussman, E. Encapsulation of bacteria and viruses in electrospun nanofibres. Nanotechnology 17, 4675-4681, doi:10.1088/0957-4484/17/18/025 (2006).

43 Alves, D. et al. Bacteriophage $\phi I B B-P F 7 A$ loaded on sodium alginate-based films to prevent microbial meat spoilage. International Journal of Food Microbiology 291, 121-127, doi:https://doi.org/10.1016/j.ijfoodmicro.2018.11.026 (2019).

44 Agarwal, R. et al. Inhaled bacteriophage-loaded polymeric microparticles ameliorate acute lung infections. Nature Biomedical Engineering 2, 841-849, doi:10.1038/s41551-018-0263-5 (2018).

45 Fortier, L.-C. \& Moineau, S. Vol. 501 203-219 (2009).

46 Ma, Y. et al. Microencapsulation of bacteriophage felix O1 into chitosan-alginate microspheres for oral delivery. Appl Environ Microbiol 74, 4799-4805, doi:10.1128/AEM.00246-08 (2008).

47 Matinkhoo, S., Lynch, K. H., Dennis, J. J., Finlay, W. H. \& Vehring, R. Spraydried Respirable Powders Containing Bacteriophages for the Treatment of Pulmonary Infections. Journal of pharmacentical sciences 100, 5197-5205, doi:https://doi.org/10.1002/jps.22715 (2011).

48 Chang, R. Y. et al. Production of highly stable spray dried phage formulations for treatment of Pseudomonas aeruginosa lung infection. European journal of pharmacentics and biopharmacentics : official journal of Arbeitsgemeinschaft fur Pharmazentische Verfahrenstechnik e.V 121, 1-13, doi:10.1016/j.ejpb.2017.09.002 (2017).

49 Leung, S. S. Y. et al. Production of Inhalation Phage Powders Using Spray Freeze Drying and Spray Drying Techniques for Treatment of Respiratory Infections. Pharm Res-Dordr 33, 1486-1496, doi:10.1007/s11095-016-1892-6 (2016).

50 Zhai, S. et al. Effect of freezing rates and excipients on the infectivity of a live viral vaccine during lyophilization. Biotechnology progress 20, 1113-1120, doi:10.1021/bp034362x (2004).

51 Ma, Y. et al. Enhanced alginate microspheres as means of oral delivery of bacteriophage for reducing Staphylococcus aureus intestinal carriage. Food Hydrocolloids 26, 434-440, doi:https://doi.org/10.1016/j.foodhyd.2010.11.017 (2012).

52 Balogh, B., Canteros, B. I., Stall, R. E. \& Jones, J. B. Control of Citrus Canker and Citrus Bacterial Spot with Bacteriophages. Plant Dis 92, 1048-1052, doi:10.1094/pdis-92-7-1048 (2008).

53 Malik, D. J. et al. Formulation, stabilisation and encapsulation of bacteriophage for phage therapy. Advances in Colloid and Interface Science 249, 100-133, doi:https://doi.org/10.1016/j.cis.2017.05.014 (2017).

54 Bosio, V., Islan, G., Martinez, Y. \& Castro, G. pp. 1-13. (2011).

55 Smidsrød, O. \& Skja k-Brtk, G. Alginate as immobilization matrix for cells. Trends in Biotechnology 8, 71-78, doi:https://doi.org/10.1016/01677799(90)90139-O (1990).

56 Lee, K. Y. \& Mooney, D. J. Alginate: properties and biomedical applications. Prog Polym Sci 37, 106-126, doi:10.1016/j.progpolymsci.2011.06.003 (2012).

57 Drury, J. L., Dennis, R. G. \& Mooney, D. J. The tensile properties of alginate hydrogels. Biomaterials 25, 3187-3199, doi:https://doi.org/10.1016/i.biomaterials.2003.10.002 (2004). 

Calcium Alginate-Chitosan Beads Containing Celecoxib Solubilized in a SelfEmulsifying Phase. Scientifica 2016, 8, doi:10.1155/2016/5062706 (2016). Gombotz, W. R. Calcium-alginate beads for the oral delivery of transforming growth factor- $\beta 1$ (TGF- $\beta 1$ ): stabilization of TGF- $\beta 1$ by the addition of polyacrylic acid within acid-treated beads. Journal of Controlled Release 30, 241-251 (1994).

60 Colom, J. et al. Microencapsulation with alginate/CaCO3: A strategy for improved phage therapy. Scientific Reports 7, 41441, doi:10.1038/srep41441 (2017).

61 Segale, L., Giovannelli, L., Mannina, P. \& Pattarino, F. Calcium Alginate and Calcium Alginate-Chitosan Beads Containing Celecoxib Solubilized in a SelfEmulsifying Phase. Scientifica 2016, 5062706-5062706, doi:10.1155/2016/5062706 (2016).

62 Chatain-Ly, M. H., Moussaoui, S., Rigobello, V., Demarigny, Y. \& Vera, A. Antiviral effect of cationic compounds on bacteriophages. Front Microbiol 4, doi:10.3389/fmicb.2013.00046 (2013).

63 Vinner, G. K., Vladisavljević, G. T., Clokie, M. R. J. \& Malik, D. J. Microencapsulation of Clostridium difficile specific bacteriophages using microfluidic glass capillary devices for colon delivery using $\mathrm{pH}$ triggered release. PLoS One 12, e0186239-e0186239, doi:10.1371/journal.pone.0186239 (2017).

64 Rayment, P. et al. Investigation of alginate beads for gastro-intestinal functionality, Part 1: In vitro characterisation. Food Hydrocolloids 23, 816-822, doi:https://doi.org/10.1016/i.foodhyd.2008.04.011 (2009).

Rubalskii, E. et al. Fibrin glue as a local drug-delivery system for bacteriophage PA5. Scientific Reports 9, 2091, doi:10.1038/s41598-018-38318-4 (2019).

Jault, P. et al. Efficacy and tolerability of a cocktail of bacteriophages to treat burn wounds infected by Pseudomonas aeruginosa (PhagoBurn): a randomised, controlled, double-blind phase 1/2 trial. The Lancet Infectious Diseases 19, 35-45, doi:https://doi.org/10.1016/S1473-3099(18)30482-1 (2019).

67 Barros, J. A. R. et al. Encapsulated bacteriophages in alginatenanohydroxyapatite hydrogel as a novel delivery system to prevent orthopedic implant-associated infections. Nanomedicine: Nanotechnology, Biology and Medicine 24, 102145, doi:https://doi.org/10.1016/j.nano.2019.102145 (2020). Bean, J. E. et al. Triggered Release of Bacteriophage $\mathrm{K}$ from Agarose/Hyaluronan Hydrogel Matrixes by Staphylococcus aureus Virulence Factors. Chemistry of Materials 26, 7201-7208, doi:10.1021/cm503974g (2014). Cobb, L. H. et al. CRISPR-Cas9 modified bacteriophage for treatment of Staphylococcus aureus induced osteomyelitis and soft tissue infection. PLoS One 14, e0220421-e0220421, doi:10.1371/journal.pone.0220421 (2019).

70 Small-Scale Preparation of Filamentous Bacteriophage by PEG Precipitation, $<$ http://www.abdesignlabs.com/technical-resources/bacteriophagepreparation/> (2015).

71 Castro-Mejía, J. L. et al. Optimizing protocols for extraction of bacteriophages prior to metagenomic analyses of phage communities in the human gut. Microbiome 3, 64-64, doi:10.1186/s40168-015-0131-4 (2015). 
72 in Methods in Enzymology Vol. 328 (eds Jeremy Thorner, Scott D. Emr, \& John N. Abelson) xv-xxxiv (Academic Press, 2000).

73 Korehei, R. \& Kadla, J. F. Encapsulation of T4 bacteriophage in electrospun poly(ethylene oxide)/cellulose diacetate fibers. Carbohydrate Polymers 100, 150157, doi:https://doi.org/10.1016/j.carbpol.2013.03.079 (2014).

74 Linse, P. Micellization of poly(ethylene oxide)-poly(propylene oxide) block copolymers in aqueous solution. Macromolecules 26, 4437-4449, doi:10.1021/ma00069a007 (1993).

75 Shlezinger, M., Friedman, M., Houri-Haddad, Y., Hazan, R. \& Beyth, N. Phages in a thermoreversible sustained-release formulation targeting $\mathrm{E}$. faecalis in vitro and in vivo. PLoS One 14, e0219599, doi:10.1371/journal.pone.0219599 (2019).

76 Kaur, S., Harjai, K. \& Chhibber, S. Bacteriophage mediated killing of Staphylococcus aureus in vitro on orthopaedic $\mathrm{K}$ wires in presence of linezolid prevents implant colonization. PLoS One 9, e90411, doi:10.1371/journal.pone.0090411 (2014).

77 Kaur, S., Harjai, K. \& Chhibber, S. In Vivo Assessment of Phage and Linezolid Based Implant Coatings for Treatment of Methicillin Resistant S. aureus (MRSA) Mediated Orthopaedic Device Related Infections. PLoS One 11, e0157626, doi:10.1371/journal.pone.0157626 (2016).

78 Wroe, J. A., Johnson, C. T. \& García, A. J. Bacteriophage delivering hydrogels reduce biofilm formation in vitro and infection in vivo. Journal of Biomedical Materials Research Part A 108, 39-49, doi:10.1002/jbm.a.36790 (2020).

79 Nieth, A., Verseux, C. \& Römer, W. A Question of Attire: Dressing Up Bacteriophage Therapy for the Battle Against Antibiotic-Resistant Intracellular Bacteria. Springer Science Reviews 3, 1-11, doi:10.1007/s40362-014-0027-x (2015).

80 Torchilin, V. P. Recent advances with liposomes as pharmaceutical carriers. Nature Reviews Drug Discovery 4, 145-160, doi:10.1038/nrd1632 (2005).

81 Akbarzadeh, A. et al. Liposome: classification, preparation, and applications. Nanoscale Res Lett 8, 102-102, doi:10.1186/1556-276X-8-102 (2013).

82 Gonzalez-Menendez, E. et al. Strategies to Encapsulate the Staphylococcus aureus Bacteriophage phiIPLA-RODI. Viruses 10, doi:10.3390/v10090495 (2018).

83 Cinquerrui, S., Mancuso, F., Vladisavljević, G. T., Bakker, S. E. \& Malik, D. J. Nanoencapsulation of Bacteriophages in Liposomes Prepared Using Microfluidic Hydrodynamic Flow Focusing. Front Microbiol 9, 2172-2172, doi:10.3389/fmicb.2018.02172 (2018).

84 Nieth, A., Verseux, C., Barnert, S., Süss, R. \& Römer, W. A first step toward liposome-mediated intracellular bacteriophage therapy. Expert Opin Drug Deliv 12, 1411-1424, doi:10.1517/17425247.2015.1043125 (2015).

85 Singla, S., Harjai, K., Katare, O. P. \& Chhibber, S. Encapsulation of Bacteriophage in Liposome Accentuates Its Entry in to Macrophage and Shields It from Neutralizing Antibodies. PLoS One 11, e0153777, doi:10.1371/journal.pone.0153777 (2016).

86 Chhibber, S., Kaur, J. \& Kaur, S. Liposome Entrapment of Bacteriophages Improves Wound Healing in a Diabetic Mouse MRSA Infection. Front Microbiol 9, doi:10.3389/fmicb.2018.00561 (2018). 

Phage Therapy against Salmonella spp. Appl Environ Microbiol 81, 4841-4849, doi:10.1128/AEM.00812-15 (2015).

Hornez, J.-C., Bouchart, F., Meurice, E., Descamps, M. \& Leriche, A. Synthesis and fabrication of porous calcium phosphate ceramics for antibacterial bone substitutes. MATEC Web of Conferences 7, 04019, doi:10.1051/matecconf/20130704019 (2013).

Fulgione, A. et al. Biomimetic hydroxyapatite nanocrystals are an active carrier for salmonella bacteriophages. International Journal of Nanomedicine 14, 2219-2232, doi:10.2147/IJN.S190188 (2019).

90 Dusane, D. H. et al. Targeting intracellular Staphylococcus aureus to lower recurrence of orthopaedic infection. Journal of Orthopaedic Research 36, 1086-1092, doi:10.1002/jor.23723 (2018).

91 Li, L. L. et al. Enhanced biofilm penetration for microbial control by polyvalent phages conjugated with magnetic colloidal nanoparticle clusters (CNCs). Environmental Science: Nano 4, 1817-1826, doi:10.1039/c7en00414a (2017).

92 Hosseinidoust, Z., Van de Ven, T. G. \& Tufenkji, N. Bacterial capture efficiency and antimicrobial activity of phage-functionalized model surfaces. Langmuir 27, 5472-5480, doi:10.1021/la200102z (2011).

93 Hornez, J. C., Bouchart, F., Meurice, E., Descamps, M. \& Leriche, A. Synthesis and fabrication of porous calcium phosphate ceramics for antibacterial bone substitutes. MATEC Web of Conferences 7 (2013).

94 Johnson, C. T. et al. Hydrogel delivery of lysostaphin eliminates orthopedic implant infection by Staphylococcus aureus and supports fracture healing. Proc Natl Acad Sci U S A 115, E4960-e4969, doi:10.1073/pnas.1801013115 (2018).

95 Hathaway, H. et al. Thermally triggered release of the bacteriophage endolysin CHAPK and the bacteriocin lysostaphin for the control of methicillin resistant Staphylococcus aureus (MRSA). Journal of controlled release : official journal of the Controlled Release Society 245, 108-115, doi:10.1016/j.jconrel.2016.11.030 (2017).

96 Kumaran, D. et al. Does Treatment Order Matter? Investigating the Ability of Bacteriophage to Augment Antibiotic Activity against Staphylococcus aureus Biofilms. Front Microbiol 9, doi:10.3389/fmicb.2018.00127 (2018).

97 Dickey, J. \& Perrot, V. Adjunct phage treatment enhances the effectiveness of low antibiotic concentration against Staphylococcus aureus biofilms in vitro. PLoS One 14, e0209390-e0209390, doi:10.1371/journal.pone.0209390 (2019).

98 Silhavy, T. J., Kahne, D. \& Walker, S. The bacterial cell envelope. Cold Spring Harb Perspect Biol 2, a000414-a000414, doi:10.1101/cshperspect.a000414 (2010).

99 Schmelcher, M., Donovan, D. M. \& Loessner, M. J. Bacteriophage endolysins as novel antimicrobials. Future Microbiology 7, 1147-1171, doi:10.2217/fmb.12.97 (2012).

100 Briers, Y. et al. Art-175 is a highly efficient antibacterial against multidrugresistant strains and persisters of Pseudomonas aeruginosa. Antimicrobial agents and chemotherapy 58, 3774-3784, doi:10.1128/aac.02668-14 (2014).

101 Fischetti, V. A. Bacteriophage lytic enzymes: novel anti-infectives. Trends in Microbiology 13, 491-496, doi:10.1016/j.tim.2005.08.007 (2005).

102 Loeffler, J. M., Nelson, D. \& Fischetti, V. A. Rapid killing of Streptococcus pneumoniae with a bacteriophage cell wall hydrolase. Science (New York, N.Y.) 294, 2170-2172, doi:10.1126/science.1066869 (2001). 
103 Schuch, R., Nelson, D. \& Fischetti, V. A. A bacteriolytic agent that detects and kills Bacillus anthracis. Nature 418, 884-889, doi:10.1038/nature01026 (2002).

104 Pastagia, M. et al. A novel chimeric lysin shows superiority to mupirocin for skin decolonization of methicillin-resistant and -sensitive Staphylococcus aureus strains. Antimicrobial agents and chemotherapy 55, 738-744, doi:10.1128/aac.0089010 (2011).

105 Filatova, L. Y. et al. Physicochemical characterization of the staphylolytic LysK enzyme in complexes with polycationic polymers as a potent antimicrobial. Biochimie 95, 1689-1696, doi:10.1016/j.biochi.2013.04.013 (2013).

106 Walsh, S., Shah, A. \& Mond, J. Improved pharmacokinetics and reduced antibody reactivity of lysostaphin conjugated to polyethylene glycol. Antimicrobial agents and chemotherapy 47, 554-558, doi:10.1128/aac.47.2.554558.2003 (2003).

107 Nithya, S. et al. Preparation, characterization and efficacy of lysostaphinchitosan gel against Staphylococcus aureus. International journal of biological macromolecules 110, 157-166, doi:10.1016/j.ijbiomac.2018.01.083 (2018).

108 Johnson, C. T. et al. Lysostaphin and BMP-2 co-delivery reduces \&lt;em\&gt;S. aureus\&lt;/em\&gt; infection and regenerates critical-sized segmental bone defects. Science Advances 5, eaaw1228, doi:10.1126/sciadv.aaw1228 (2019).

109 Gutierrez, D., Ruas-Madiedo, P., Martinez, B., Rodriguez, A. \& Garcia, P. Effective removal of staphylococcal biofilms by the endolysin LysH5. PLoS One 9, e107307, doi:10.1371/journal.pone.0107307 (2014).

110 Olsen, N. M. C. et al. Synergistic Removal of Static and Dynamic Staphylococcus aureus Biofilms by Combined Treatment with a Bacteriophage Endolysin and a Polysaccharide Depolymerase. Viruses 10, doi:10.3390/v10080438 (2018).

111 Pennone, V. et al. Inhibition of L. monocytogenes Biofilm Formation by the Amidase Domain of the Phage vB_LmoS_293 Endolysin. Viruses 11, 722, doi:10.3390/v11080722 (2019). 


\section{Chapter 7}

\section{Local delivery of bacteriophages by thermoresponsive HA- pNIPAM hydrogels and alginate-chitosan microbeads as a treatment for infection}

S.G. Rotman ${ }^{1,2}$, V. Post $^{1}$, A. Foster ${ }^{1,3,4}$, T.F. Moriarty ${ }^{1}$, D.W. Grijpma ${ }^{2}$, D. Eglin ${ }^{1}$

${ }^{1}$ AO Research Institute Davos, Davos Platz, Switzerland

${ }^{2}$ Department of Biomaterials Science and Technology, Faculty of Science and Technology, University of Twente, Enschede, The Netherlands

${ }^{3}$ Faculty of Science and Engineering, Queensland University of Technology (QUT), Brisbane, Australia.

${ }^{4}$ Department of Orthopaedic Surgery, Royal Brisbane and Women's Hospital, Queensland, Australia 


\section{Abstract}

Against a background of increasing incidence of antibiotic resistant infections, bacteriophages are emerging as an appealing antibacterial alternative. Bacteriophage treatment of orthopedic infections often relies on repeated local administration of phage suspensions over several days through intraoperative irrigation followed by regular instillation through percutaneous tubing that is left in situ for the entire duration. This approach has been successful in early reports; however, it requires extensive in-hospital treatment and the instillation tube is a significant risk factor for super infection. To avoid the need for repeated local bacteriophage administration, thermoresponsive hyaluronic acid-poly( $N$-isopropylacrylamide) (HApNIPAM) hydrogels and alginate-chitosan microbeads have been developed as local bacteriophage delivery systems. Staphylococcus aureus phage ISP and Pseudomonas aeruginosa phage LUZ19 were embedded in HA-pNIPAM hydrogels and alginate-chitosan microbeads and their lytic activity and release from the biomaterials was monitored. Rapid release of $10^{8}-10^{9}$ PFU/mL ISP and LUZ19 from HA-pNIPAM hydrogels was gradually reduced to $10^{5}-10^{7} \mathrm{PFU} / \mathrm{mL}$ after 21 days. When phage-loaded alginatechitosan microbeads were combined with phage loaded HA-pNIPAM, sustained release of high titers of LUZ19 was observed, while this was not observed for ISP, suggesting that release kinetics are phage dependent. Both ISP and LUZ19 bacteriophages were released in a relevant dosage and over a period of time that matches current clinical use, therefore highlighting the potential of HA-pNIPAM hydrogels and alginate-chitosan microbeads for local delivery of bacteriophages. 


\section{Introduction}

Bacteriophages are viruses that can selectively infect and lyse bacteria in a rapid manner and can act as an alternative for antibiotics in infection prevention and treatment, even against multiple antibiotic-resistant pathogens. While the discovery of bacteriophages predates the development of antibiotics, research on bacteriophages was mostly abandoned after antibiotics were mass-produced in the 1940's. A main stimulus for the renewed interest in phage therapy seen in recent years is the growing problem of antibiotic resistant bacteria [1]. Bacteriophages are not affected by the antibiotic-resistance mechanisms and phage-resistance is a relatively rare occurrence which might be overcome by co-evolution of the bacteriophages themselves [2,3].

Bacteriophages could in principle be utilized as antimicrobials in many scenario's where antibiotics are currently being used. Self-replication occurs during after the bacteriophage inserts its genetic material into the bacterial cell during the lytic cycle. This presents the advantageous property of selfdosing, as without host bacteria the phages lose their ability to replicate. However, in practice the formation of new bacteriophages is counterbalanced by in vivo bacteriophage clearance by the patient's immune system, resulting in a declining bacteriophage presence over time. This necessitates repeated administration of bacteriophages in order to achieve successful treatment outcomes. While repeated topical or oral administration of bacteriophage is not very challenging to achieve, it does add complexity to infection treatment of internal tissues (e.g. bone infections). Bacteriophage therapy for such infections would benefit greatly from delivery systems or 
carrier materials that allow for a continuous release of bacteriophages locally at the site of infection.

Carrier materials for local delivery of bacteriophages has recently been reviewed in Chapter 5. According to literature reviewed, hydrogels (either derived from natural or synthetic polymers) make for a highly suitable material class for the embedding and subsequent release of bacteriophages. Our group has previously reported on the synthesis of a semi-natural thermo-responsive hyaluronic acid-poly( $\mathrm{N}$-isopropylacrylamide) (HApNIPAM) hydrogel that is suitable as a carrier for human mesenchymal stem cells (hMSCs) [4] or as a drug delivery system for water soluble antibiotics [5]. This work aims to assess the compatibility of bacteriophages embedded in HA-pNIPAM and the potential of HA-pNIPAM hydrogels as a delivery system for Staphylococcus aureus (S. aureus) phage ISP and Pseudomonas aeruginosa (P. aeruginosa) phage LUZ19. Structural differences between long-tailed ISP phages (caudoviralis family) and short-tailed LUZ19 phages (podoviridae family) might alter phage diffusion rates out of a hydrogel, which would be worthy of investigation. As a second biomaterial, alginate and chitosan were used for embedding ISP and LUZ19. Extensive work on alginate and chitosan materials for bacteriophage delivery was reported and these materials are considered highly compatible with bacteriophages with potential applications in food processing [6], animal husbandry [7] and gastrointestinal drug delivery [8]. Bacteriophage delivery with alginate-chitosan microbeads for internal medicine, such as orthopedic infections, has not been previously reported.

In this work, ISP and LUZ19 were used in phage release studies. These phages show specific activities against $S$. aureus and $P$. aeruginosa which are 
commonly identified in orthopaedic infections. Release of ISP and LUZ19 phages from thermoresponsive HA-pNIPAM, alginate-chitosan microbeads and combinations of these two biomaterials is investigated.

\section{Materials and Methods}

\section{Materials}

HA sodium salt was acquired from Contipro Biotech (Czech Republic), number average molecular weight $\left(\mathrm{M}_{\mathrm{n}}\right)=170.6 \mathrm{kDa}$ and polydispersity $\left(\mathrm{M}_{\mathrm{w}} / \mathrm{M}_{\mathrm{n}}\right)=1.73$. $\mathrm{N}$-isopropylacrylamide, dimethylformamide, cysteamine hydrochloride, diethylether, sodium bromide, calcium chloride $\left(\mathrm{CaCl}_{2}\right)$, Chitosan, anhydrous acetic acid, sodium chloride, anhydrous magnesium sulfate, sodium bicarbonate, Dowex Resin M-31, Carbonyl diimidazole and phosphate buffer solution (PBS) tablets were purchased from Sigma Aldrich (St. Louis, MO, USA). Tetrabutylammonium (TBA), methanesulfonic acid, 2,2'-azobis(2-methylpropionitrile (AIBN) and Tris-(hydroxymethyl)aminomethane hydrochloride (TRIS-HCl) was bought from Fluka (Buchs, Switzerland). Alginate (LVG, high G content) was purchased from Nova Matrix (Sandvika, Norway). Sodium citrate was acquired from Carl Roth (Karlsruhe, Germany). Bacteriological agar, Tryptic soy agar (TSA) plates and Tryptic soy broth (TSB) were purchased from Oxoid AG (Basel, Switzerland). Bacterial strain Staphylococcus aureus JAR (CCOS, Wädenswill, Switzerland) was used. Pseudomonas aeruginosa PA01 and LUZ19 phage were kindly donated by dr. Wagemans, KU Leuven, Belgium. Staphylococcus phage ISP was kindly donated by dr. Merabishvili, Queen Mary Military Hospital Brussels, Belgium. 


\section{pNIPAM-NH ${ }_{2}$ synthesis}

The synthesis of amine terminated poly(N-isopropylacrylamide) (pNIPAM$\mathrm{NH}_{2}$ ) was done according to ter Boo et al. [5] Briefly, 10 gram (88.4 mmol) of NIPAM monomer was dissolved in $20 \mathrm{~mL}$ of $\mathrm{N}_{2}$ degassed DMF. The NIPAM solution was brought under an inert atmosphere by further $\mathrm{N}_{2}$ degassing. Next, $100 \mathrm{mg}$ of Cysteamine hydrochloride and $15 \mathrm{mg}$ AIBN were added to the NIPAM solution. The solution was heated to $70^{\circ} \mathrm{C}$ and NIPAM polymerization occurred for 7 hours under continued $\mathrm{N}_{2}$ bubbling. The pNIPAM-NH $\mathrm{N}_{2}$ solution was cooled down to $\mathrm{RT}$ and was precipitated in diethylether. The precipitate was vacuum-filtered and washed with diethylether, followed by 3 days of desiccation under vacuum.

\section{HA-pNIPAM synthesis}

The synthesis of HA-pNIPAM was adapted from D'Este et al. [9] Briefly, HA sodium salts were converted tetrabutylammonium (TBA) salts by ionexchange of $\mathrm{Na}^{+}$and $\mathrm{TBA}^{+}$ions using Dowex Resin M-31. HA-TBA was lyophilized and $2.0 \mathrm{~g}$ of dried HA-TBA was dissolved in $160 \mathrm{~mL}$ DMSO. Separately, $3.5 \mathrm{~g}$ of pNIPAM-NH $\mathrm{N}_{2}$ was dissolved in $40 \mathrm{~mL}$ of DMSO. To the dissolved HA-TBA, $80 \mu \mathrm{L}$ of Methanesulfonic acid and $540 \mathrm{mg}$ Carbonyl diimidazole are added and stirred at $42^{\circ} \mathrm{C}$ in an oil bath for 1 hour. The pNIPAM-NH $\mathrm{N}_{2}$ solution is added to the HA-TBA solution and the mixture is stirred at room temperature for 48 hours. $20 \mathrm{~mL}$ of sodium bromide solution $(930 \mathrm{~g} / \mathrm{L})$ was added dropwise to the reaction mixture and was stirred for 2 hours. The solution was dialyzed against cold tap water and was changed regularly. After 5 days, the HA-pNIPAM solution was lyophilized. 


\section{Preparation of Alginate and Alginate-Chitosan microbeads}

A solution of alginate (high $\mathrm{G}$ content) was made by dissolution of $60 \mathrm{mg}$ in 3.6 $\mathrm{mL}$ of MilliQ $\mathrm{H}_{2} \mathrm{O}$. After complete dissolution of the alginate, $0.4 \mathrm{~mL}$ of ISP or LUZ19-phage stock $\left(10^{10}-10^{11} \mathrm{PFU} / \mathrm{mL}\right)$ was added bringing the total alginate concentration to $1.5 \% \mathrm{w} / \mathrm{v}$. The Alginate solution containing phage was electrosprayed using a pressure over fluid system (Spraybase, Kildare, Ireland) with a pressure of $1 \mathrm{bar}$, emitter voltage of $10 \mathrm{kV}$, spray height of $10 \mathrm{~cm}$ and a 27 gauge needle. The solution was sprayed in a collection bath containing $30 \mathrm{~mL}$ of $100 \mathrm{mM} \mathrm{CaCl}$. After the complete volume of the alginate/phage solution was sprayed, the solidified alginate microbeads were filtered using vacuum-assisted filtration on filter paper with mesh size 3 and washed with MilliQ $\mathrm{H}_{2} \mathrm{O}$ to remove excess $\mathrm{CaCl}_{2}$. To fabricate alginatechitosan microbeads, the alginate microbeads were further processed. A Chitosan solution containing $4 \mathrm{mg} / \mathrm{mL}$ Chitosan in 2\% Acetic acid was made and its $\mathrm{pH}$ was adjusted to 7.5. The collected phage loaded alginate microbeads were dispersed in $20 \mathrm{~mL}$ of chitosan solution and dispersed by vortex. The alginate microbeads were stirred in the Chitosan solution for 10 minutes, after which the microbeads were filtered and washed as mentioned earlier. The alginate-chitosan microbeads were kept in airtight containers at $4^{\circ} \mathrm{C}$ until further use. Imaging of alginate and alginate-chitosan microbeads was done by light microscopy (AxioVert A1, Zeis, Oberkochen, Germany) using Axiovision4 software. The alginate or alginate-chitosan microbeads were always used within 24 hours after the electrospray procedure.

\section{Rheological properties of phage-loaded HA-pNIPAM hydrogels}

Hydrogels with and without ISP or LUZ19 phage load were tested to investigate changes in gelation kinetics and network properties caused by the 
presence of bacteriophages. Rheological changes of the HA-pNIPAM hydrogel over a temperature range of $20-40^{\circ} \mathrm{C}$ was assessed with the Anton Paar Rheometer MCR302. The hydrogel was applied on the rheometer bottom platform and a cone plate $25 \mathrm{~mm}$ (CP25) was used as a mobile top plate. The gap between the two surfaces during measurement was $50 \mu \mathrm{m}$. To assess the thermoresponsive mechanical properties of HA-pNIPAM, the temperature increased with approximately $1{ }^{\circ} \mathrm{C} / \mathrm{min}$ and a $0.5 \%$ strain was applied at a frequency of $1 \mathrm{~Hz}$.

\section{Preparation of buffers and bacteriological agar}

The buffer most frequently used in bacteriophage experimental work contains sodium and magnesium ions and is therefore often called SMbuffer. SM-buffer was prepared by adding 5.8 gram sodium chloride, 1 gram anhydrous magnesium sulfate and $50 \mathrm{~mL}$ of $1 \mathrm{M}$ Tris- $\mathrm{HCl}$ solution at $\mathrm{pH} 7.4$

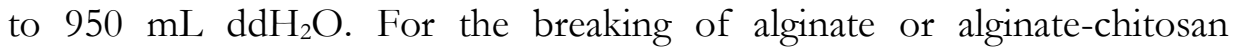
microbeads, $200 \mathrm{~mL}$ bead-breaker solution was prepared containing $50 \mathrm{mM}$ sodium citrate, $0.2 \mathrm{M}$ sodium bicarbonate, and $50 \mathrm{mM}$ Tris- $\mathrm{HCl}$ solution at $\mathrm{pH}$ 7.5. Plaque assays of ISP and LUZ19 phages required slightly different agar solutions due to the large plaque size of LUZ19. Bacteriological agar was prepared by adding 1.2 gram (ISP) or 1.6 gram (LUZ19) agar and 6 gram

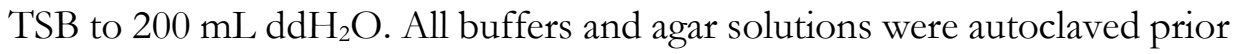
to use.

\section{Bacteriophage stability in HA-pNIPAM hydrogels}

HA-pNIPAM hydrogels were made by preparing a $10 \% \mathrm{w} / \mathrm{v}$ solution with SM-buffer containing approximately $10^{10}-10^{11} \mathrm{PFU} / \mathrm{mL}$. In quintuplicate $(\mathrm{n}=5), 200 \mu \mathrm{L}$ of the phage loaded hydrogel was pipetted into glass vials 
which were stored at either 4 or $37^{\circ} \mathrm{C}$. After 1, 3, 7, 11 and 14 days, a single vial was taken and the phage titer inside the hydrogel was determined. For hydrogels incubated at $37^{\circ} \mathrm{C}$, the vials were briefly kept in the fridge to allow phase transition of the HA-pNIPAM gel. Control groups included phages kept in buffer. To assess potential adverse effects of HA-pNIPAM gelation on phage activity, phage loaded hydrogels were incubated and cooled in repeated cycles of 5 minutes. Phage titers were assessed after 0,1 and 3 gelation cycles by plaque assay. During plaque assays, $100 \mu \mathrm{L}$ of appropriately diluted phage sustention was added to $100 \mu \mathrm{L}$ of bacterial suspension and 4 $\mathrm{mL}$ of liquid bacteriological agar in a $15 \mathrm{~mL}$ Falcon tube. The tube was gently mixed and the liquid bacteriological agar containing phages and bacteria were poured on TSA plates. Plaques were counted after the plates were incubated for 16-24 hours.

\section{Bacteriophage release studies}

\section{Bacteriophage release from $H A-p N I P A M$}

Lyophilized HA-pNIPAM was reconstituted at a concentration of $10 \% \mathrm{w} / \mathrm{v}$ with SM-buffer containing approximately $10^{10}-10^{11} \mathrm{PFU} / \mathrm{mL}$. Reconstitution occurred overnight at $4^{\circ} \mathrm{C}$ under mild rotation. Aliquots of $800 \mu \mathrm{L}$ of phage loaded HA-pNIPAM were made in glass vials and the hydrogel could solidify at $37^{\circ} \mathrm{C}$ for 30 minutes, after which $3.2 \mathrm{~mL}$ of SM buffer was added on top of the gels. The complete release buffer was aspirated and refreshed every day for 21 days. After 21 days, the hydrogels were cooled in the fridge and phage remaining in the gels was quantified. Phage quantification of the release media and endpoint hydrogels was performed by plaque assay as described above. 


\section{Bacteriophage release from alginate and alginate-chitosan microbeads}

Alginate and alginate-chitosan microbeads were weighed and dispersed in 5 $\mathrm{mL} \mathrm{SM}$ buffer at a concentration of $10 \mathrm{mg} / \mathrm{mL}$ and $30 \mathrm{mg} / \mathrm{mL}$ respectively. The sample were incubated at $37^{\circ} \mathrm{C}$ and after 1, 2, 3 and 4 days, $100 \mu \mathrm{L}$ was withdrawn and further diluted for phage quantification by plaque assay. After 4 days, $5 \mathrm{~mL}$ of bead-breaker solution was added to the samples, bringing the total volume up to approximately $10 \mathrm{~mL}$. The alginate or alginatechitosan microbeads were allowed to dissolve, and remaining phage was quantified by plaque assay as described above.

Bacteriophage release from combinations of $H A-p N I P A M$ and alginate-chitosan microbeads

Phage loaded alginate and alginate-chitosan microbeads were weighed (50 $\mathrm{mg}$ and $150 \mathrm{mg}$, respectively) in glass vials. Next, $800 \mu \mathrm{L}$ of phage loaded $10 \% \mathrm{w} / \mathrm{v}$ HA-pNIPAM containing $10^{9}-10^{10} \mathrm{PFU} / \mathrm{mL}$ was added to the vials with the microbeads. The microbeads and hydrogel were gently stirred by pipette tip and by mild vortex. The samples were incubated at $37^{\circ} \mathrm{C}$ for 30 minutes to allow gelation of the HA-pNIPAM and $3.2 \mathrm{~mL}$ of SM-buffer was subsequently added to the vials. The complete release buffer was aspirated and refreshed every day for 21 days. After 21 days, the hydrogels were cooled in the fridge and $5 \mathrm{~mL}$ of bead-breaker solution was added. Phage quantification of the release media and endpoint hydrogels was performed by plaque assay as described above. 


\section{Results}

\section{${ }^{1} \mathrm{H}-\mathrm{NMR}$ analysis and rheological properties of HA-pNIPAM}

\section{hydrogels}

Analysis on the molecular structure of HA-pNIPAM copolymers can be seen in Figure 7.1A. Protons from the grafted pNIPAM polymers are labeled $\mathbf{a}$ at $1.14 \mathrm{ppm}, \mathbf{b}$ at $3.82 \mathrm{ppm}, \mathbf{c}$ at $1.96 \mathrm{ppm}$, and $\mathbf{d}$ at $1.3-1.7 \mathrm{ppm}$. The broad distribution of $\mathrm{d}$ protons was previously attributed by D'Este et al. to limited rotation of the c-d carbon atoms due to steric hindrance [9]. Protons on the HA backbone of the copolymer are labeled e at 3.2-3.6 ppm, $\mathbf{h}$ at 4.3-4.5 $\mathrm{ppm}, \mathbf{f}$ at $1.96 \mathrm{ppm}$ and $\mathbf{g}$ at $3.65 \mathrm{ppm}$. The degree of substitution (DS\%) of pNIPAM grafting was calculated by integrating the peaks from the $9 \mathrm{HA}$ protons labeled e and the peaks from pNIPAM labeled a. The DS\% of pNIPAM grafted to the HA backbone was calculated to be $6.5 \mathrm{~mol} / \mathrm{mol} \%$. Figure 7.1B shows the gelation properties of HA-pNIPAM hydrogels in absence or presence of phage load. All hydrogels, regardless of presence of ISP or LUZ19 phage, showed an equal storage modulus after complete gelation at physiological temperatures (HA-pNIPAM: 2255 $\pm 648 \mathrm{~Pa}$; HApNIPAM + ISP-phage: $1982 \pm 119 \mathrm{~Pa}$ and HA-pNIPAM + LUZ19: $1967 \pm 110 \mathrm{~Pa}$ ). At lower temperatures the presence of phages slightly increased the viscosity of the hydrogels resulting in higher storage modulus measurements. The temperature range in which gelation took place $( \pm 28$ $31^{\circ} \mathrm{C}$ ), as well as gelation kinetics were not affected by phage loads. This rheological assessment ensured that phages did not alter the thermoresponsive behavior of the HA-pNIPAM hydrogels. Practical limitations prevented rheological assessment of HA-pNIPAM with alginate- 
chitosan microbeads, due to the large diameter of the microbeads and tight gap between the plates during rheological measurements.

A.

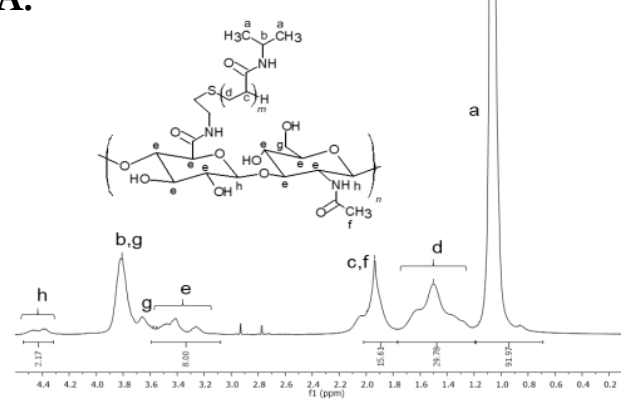

B.

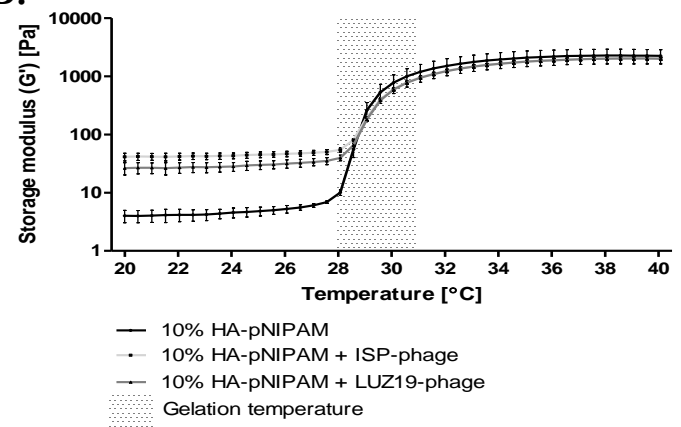

Figure 7.1 - Physicochemical characterization of HA-pNIPAM hydrogels.

A. ${ }^{1} \mathrm{H}$-NMR analysis of HA-pNIPAM. Solvent: $\mathrm{D}_{2} \mathrm{O}$

B. Rheological assessment of empty and phage-loaded HA-pNIPAM hydrogels $(n=3)$ in the temperature range of $20-40^{\circ} \mathrm{C}$.

Stability of LUZ19 and ISP phage in HA-pNIPAM hydrogels at $37^{\circ} \mathrm{C}$

HA-pNIPAM hydrogel is a novel biomaterial for phage delivery and thus phage compatibility needs to be tested. In Figure 7.2 the active phage titers present inside HA-pNIPAM hydrogels was monitored. Figure 7.2A shows the active titers of ISP phage in hydrogel and SM-buffer over a period of two weeks. Phage in SM-buffer at $4^{\circ} \mathrm{C}$ (open circles) is regarded as suitable storage condition of phages over a short to medium timespan. When the phage in buffer is kept at $37^{\circ} \mathrm{C}$, we can see that elevated temperatures gradually reduce the activity of ISP phage. Phage in HA-pNIPAM hydrogel (open squares) shows a similar, but slightly more pronounced gradual decrease in phage activity. Phage activity was not altered during the hydrogel hydration phase, which occurred in $4^{\circ} \mathrm{C}$ under slow continuous vial inversion, showing that any mechanical stresses applied during this step did not inhibited ISP phages activity. Active titers of ISP (solid line) and LUZ19 
(dotted line) phages in hydrogels kept at $37^{\circ} \mathrm{C}$ are seen in Figure 7.2B. Over a period of 2 weeks, the embedded phages are inactivated due to a combination of elevated temperatures mainly and slight effect of HApNIPAM. In this set-up, small volumes of 10\% HA-pNIPAM hydrogels were kept in glass vials and condensation could be observed on the inside of the vials. The decreasing water content in the hydrogel might have contributed to the reduction in phage activity, as a hydrated environment has been recognized as an important factor in maintaining phage activity [10]. Because a sharp reduction of $2 \log _{10}$ was observed for ISP phage activity within 24 hours, physical crosslinking of the HA-pNIPAM copolymers during gelation was observed to determine if it exerts mechanical stresses on the larger and tailed ISP phages. In Figure 7.2C the gelation of ISP loaded HA-pNIPAM hydrogels was repeated 3 times. As no decrease in phage activity can be observed in Figure 7.2C, it was ruled out that the thermally driven physical crosslinking of HA-pNIPAM gels reduced active ISP phage titers. 
A.

B.

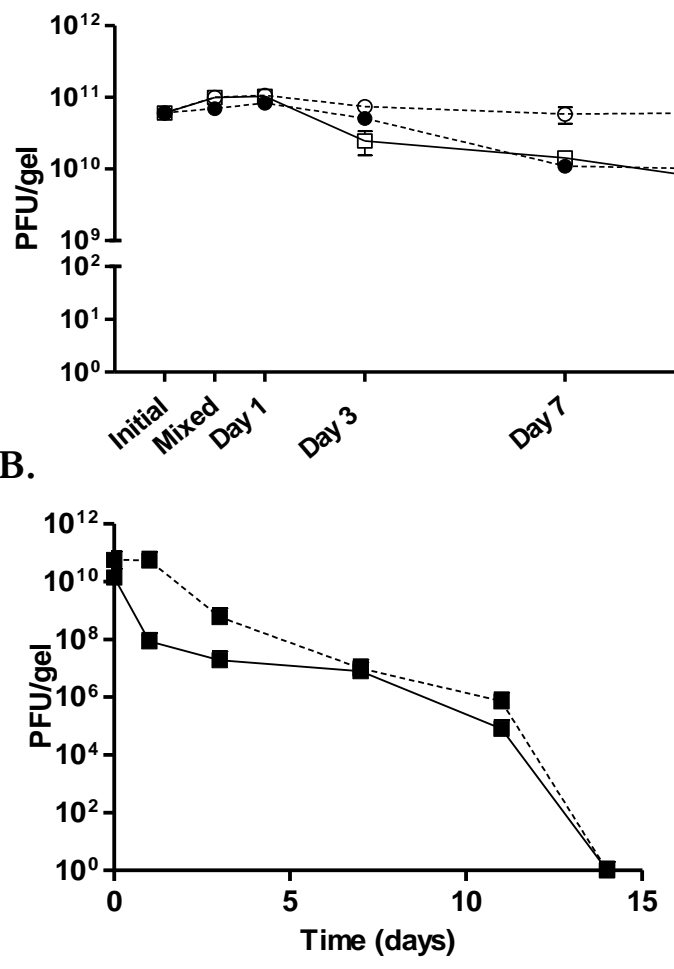

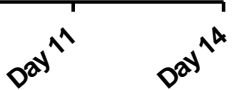

C.

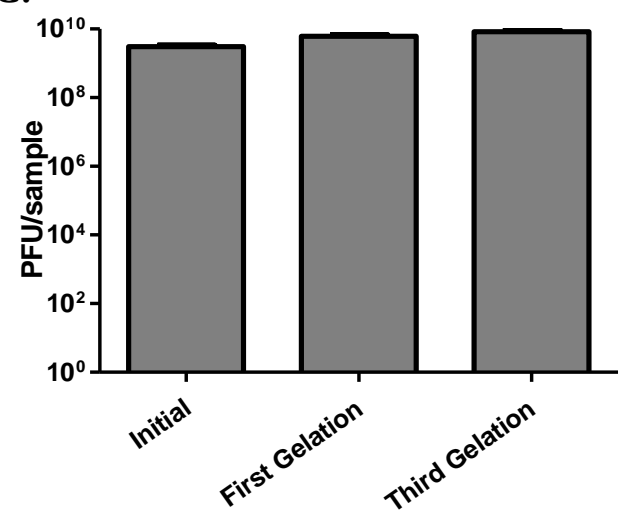

Figure 7.2 - Assessment of phage activity in HA-pNIPAM hydrogels.

A. ISP phage activity in HA-pNIPAM or SM buffer. Open circles (O) represent phage in SM-buffer kept at $4^{\circ} \mathrm{C}$, open squares $(\square)$ represent phage in HA-pNIPAM hydrogel kept at $4^{\circ} \mathrm{C}$ and filled circles $(\bullet)$ represent phage in SM-buffer incubated at $37^{\circ} \mathrm{C}$.

B. ISP and LUZ19 phage in HA-pNIPAM hydrogel incubated at $37^{\circ} \mathrm{C}$. Solid line represents ISP phage, dotted line represents LUZ19 phage.

C. Effect of repeated reversible thermal induced gelation on ISP phage activity.

\section{ISP and LUZ19 release from HA-pNIPAM hydrogels}

Figure 7.3 shows the release of ISP (A.) and LUZ19 phages (B.) from HApNIPAM hydrogels at $37^{\circ} \mathrm{C}$. The initial phage load in the HA-pNIPAM hydrogel samples differed slightly between the two experiment $\left(3.1 \cdot 10^{10}\right.$ PFU/gel for ISP and $9.8 \cdot 10^{9} \mathrm{PFU} /$ gel for LUZ19), potentially leading to the 
slightly lower initial titers observed for LUZ19 in Figure 7.3B. The cumulative release of ISP was higher ( $42 \%$ of initial phage load) compared to LUZ19 (28\% of initial phage load). However, most of the ISP release occurred within 48 hours, with released phage titers being reduced to $10^{6}$ PFU/gel at day 12 while LUZ19 phage release remained more constant over the 21 day experiment. After following the phage release for 21 days, the PFU still remaining in the HA-pNIPAM hydrogel was $1.3 \cdot 10^{6} \mathrm{PFU} /$ gel for ISP $2.2 \cdot 10^{7} \mathrm{PFU} / \mathrm{gel}$ for LUZ19. This 10 -fold difference was remarkable as it was expected that the smaller LUZ19 phage could more easily diffuse out of the HA-pNIPAM, resulting in an almost empty hydrogel. Alternatively, LUZ19 phage might be better preserved inside HA-pNIPAM compared to ISP phages due to chemical interactions with the hydrogel macromolecules. The reducing ISP and LUZ19 phage activity in the hydrogel seen in Figure 7.3B warrant alterations to the phage delivering biomaterials to increase their potential for local phage delivery. Alginate and alginate-chitosan materials were further investigated as phage-loaded additives to improve the phage release kinetics of HA-pNIPAM hydrogels. 
A.

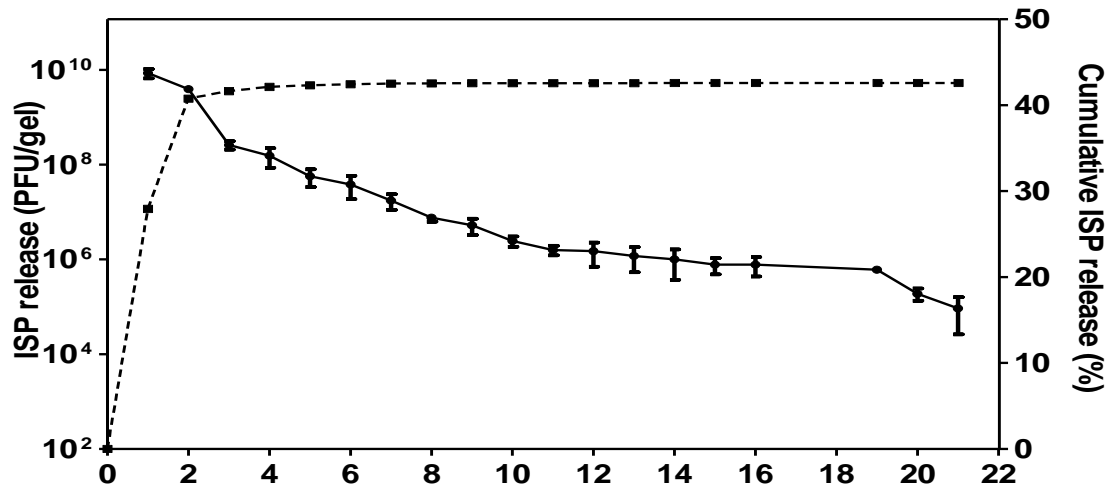

B.

Time (h)

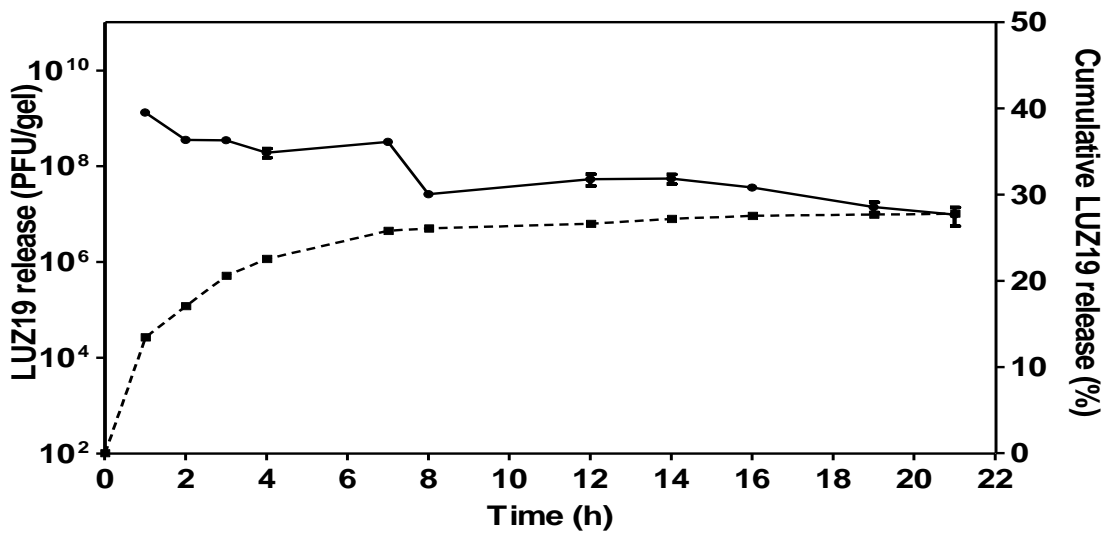

Figure 7.3 - Release profile of bacteriophages from HA-pNIPAM hydrogels at $37^{\circ} \mathrm{C}$. The solid line represents the PFU titers observed in the supernatant at specific timepoints and should be read on the left Y-axis. The dashed line illustrates the cumulative release profile up to the specific timepoints indicated and should be read on the right $\mathrm{Y}$-axis.
A. Release profile of ISP phages
B. Release profile of LUZ19 phages 


\section{Characterization of Alginate and Alginate-Chitosan microbeads}

Alginate microbeads and further processed alginate-chitosan microbeads were imaged immediately after fabrication and are depicted in Figure 7.4A and Figure 7.4B. During the chitosan deposition on the microbeads, the size average increases as can be seen on the microscopic images and Figure 7.4C which shows the average diameter of the two groups of microbeads ( $\mathrm{n}>$ 100). Because chitosan deposition occurs in wet, $\mathrm{pH}$-neutral conditions and alginate is known to swell in non-acidic environments, it was important to make sure that the increased bead diameter was not only the result of bead swelling, but also of chitosan deposition. In Figure 7.4D we observed a significant $(p=0.0084)$ increase in absorption of the amine labeling agent ophthaldialdehyde, indicating an increased presence of amine groups associated to the microbeads once alginate-chitosan microbeads were formed. 
A.

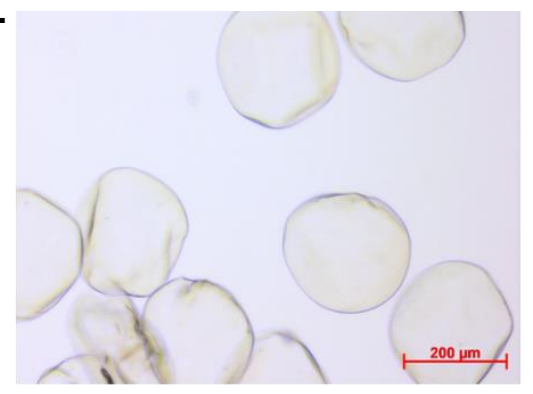

D.

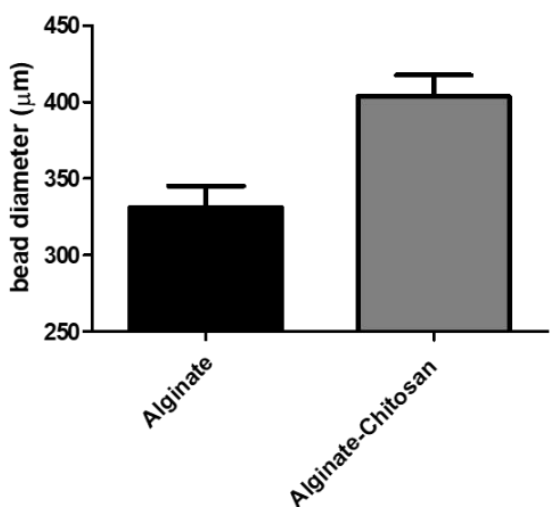

B.

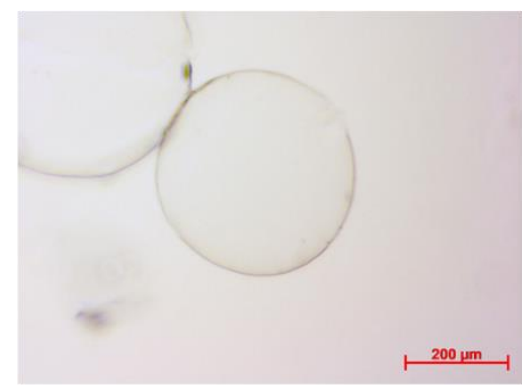

C.

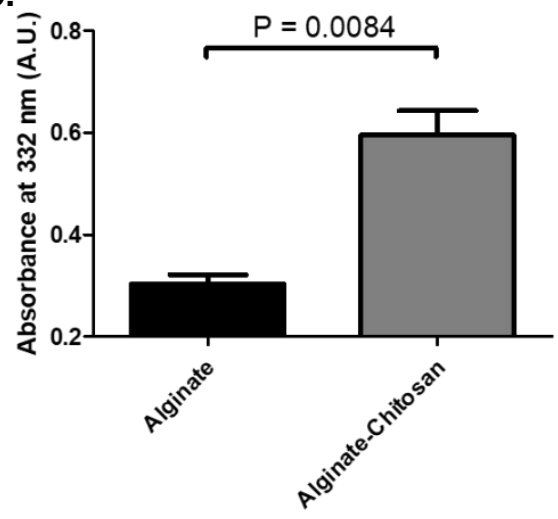

Figure 7.4 - Characterization of electrosprayed alginate and alginate-chitosan microbeads.

A Light microscopy images of alginate microbeads. Scalebar $=200 \mu \mathrm{m}$.

B. Light microscopy images of alginate-chitosan. Scalebar $=200 \mu \mathrm{m}$.

C. Average bead diameter of alginate microbeads $(n=105)$ and alginate-chitosan microbeads $(n=100)$.

D. O-phthaldialdehyde absorbance of dissolved microbeads. 


\section{ISP and LUZ19 release from Alginate and Alginate-Chitosan microbeads}

Release of ISP and LUZ19 phages from alginate and alginate-chitosan microbeads can be seen in Figure 7.5. Short release studies were planned as the alginate and alginate-chitosan microbeads were expected to swell rapidly when freely dispersed in buffer. However, the microbeads did not disintegrate throughout the experiment, meaning that longer release studies with these alginate and alginate-chitosan microbeads could theoretically be performed. Against expectations, alginate-chitosan microbeads released ISP and LUZ19 phages in higher quantities across the four-day experimental period. This might be due to the slightly swollen state of the alginate-chitosan microbeads that is achieved during the chitosan surface deposition. This looser network of the alginate core might facilitate better diffusion of the phage out of the microbeads. Release of LUZ19 from alginate increased after 48 hours, which was distinctly different compared to ISP release from alginate microbeads. While release of ISP from alginate-chitosan microbeads does not completely plateau after 24 hours, release of LUZ19 significantly increases at later timepoints. 
A.

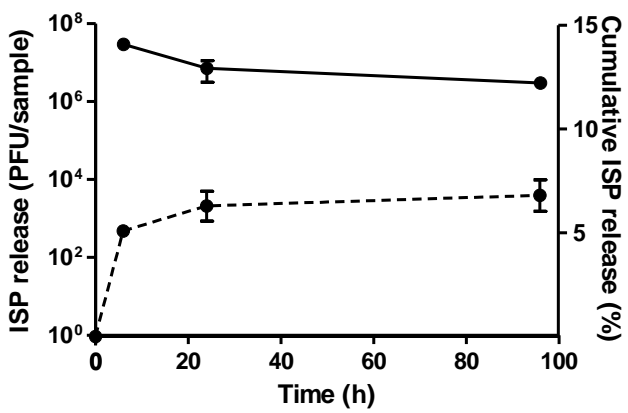

B.

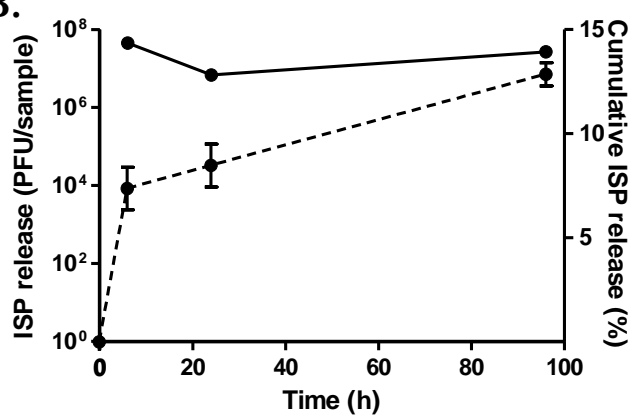

C.
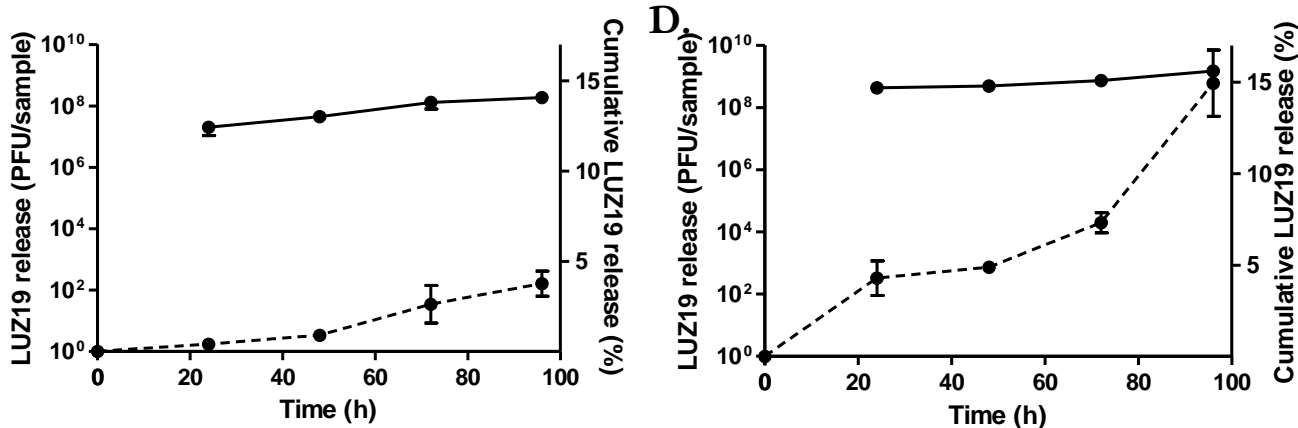

Figure 7.5 - Release profiles of ISP and LUZ19 bacteriophages from alginate or alginate-chitosan microbeads. The solid line represents the PFU titers observed in the supernatant at specific timepoints and should be read on the left Y-axis. The dashed line illustrates the cumulative release profile up to the specific timepoints indicated and should be read on the right $\mathrm{Y}$-axis.
A. ISP phage release from alginate microbeads.
B. ISP phage release from alginate-chitosan microbeads.
C. LUZ19 phage release from alginate microbeads.
D. LUZ19 phage release from alginate-chitosan microbeads.

\section{ISP and LUZ19 release from combined HA-pNIPAM hydrogel and alginate-Chitosan microbeads}

Figure 7.6 shows the release profiles of ISP and LUZ19 phages from HApNIPAM hydrogels with or without addition of alginate or alginate-chitosan microbeads. The release profile of ISP in Figure 7.6A reveals that the addition of microbeads does not affect the release kinetics over time, in contrast with Figure 7.6B, which does shows that prolonged release of high 
titers of LUZ19 phage is established after addition of LUZ19-loaded alginate-chitosan microbeads. The cumulative release profiles of ISP and LUZ19 phages can be seen in Figure 7.6C and Figure 7.6D, respectively. Due to the fact that ISP phage release from HA-pNIPAM with alginate or alginate-chitosan microbeads was higher compared to only HA-pNIPAM hydrogel over the full 21 day release experiment, the cumulative ISP phage release increased drastically by approximately $25-30 \%$. However, the ISP release profile was not affected as most phage was released during the initial burst release in the first 5 days. Interestingly, LUZ release from HApNIPAM gels with alginate-chitosan microbeads increased after day 7, with even alginate bead enriched HA-pNIPAM showing a slight increase in phage release after day 14 (Figure 7.6D). This delayed effect was also observed when the microbeads were incubated in buffer, albeit much earlier at 48 hours (Figure 7.5). This increased release of phage load shows the benefits of addition of alginate materials, with alginate-chitosan yielding the most sustained and highest in vitro LUZ19 release. 
A.

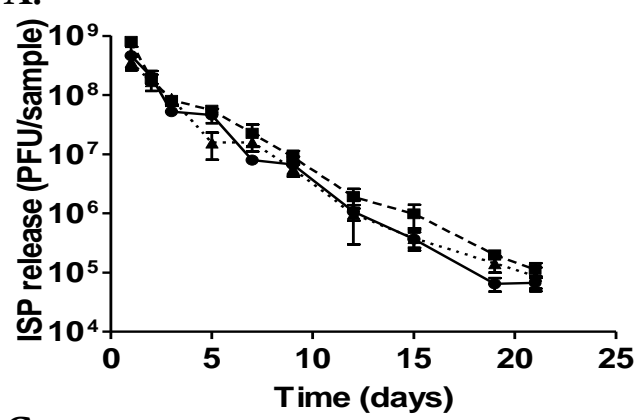

C.

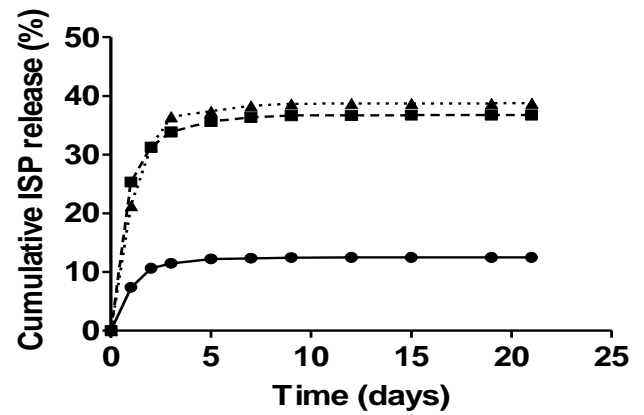

B.

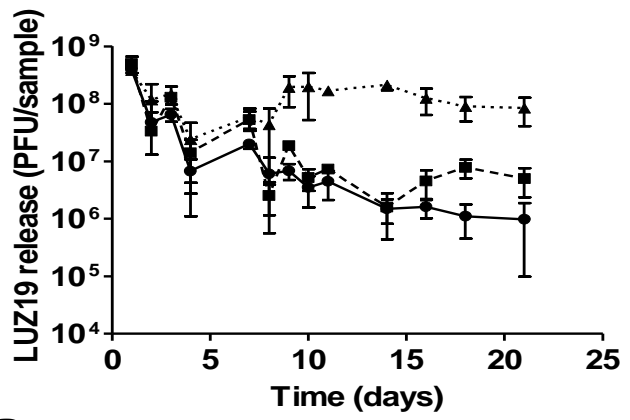

D.

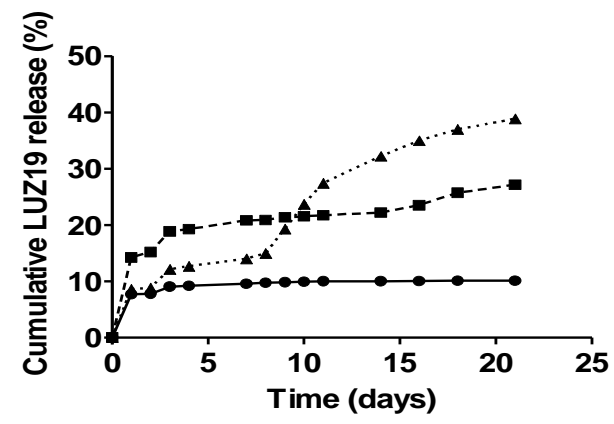

Figure 7.6 - Release profiles of ISP and LUZ19 phages from HA-pNIPAM hydrogels containing phage-loaded alginate or alginate-chitosan microbeads. Circles (•) connected with a solid line indicate phage release from HA-pNIPAM hydrogels, squares ( $\square$ ) connected with a dashed line represent phage release from HApNIPAM hydrogels with alginate microbeads and triangles $(\Delta)$ connected with a dotted line represent phage release from HA-pNIPAM hydrogels with alginatechitosan microbeads.
A. Release profile of ISP phages
B. Release profile of LUZ19 phages
C. Cumulative release profile of ISP phages
D. Cumulative release profile of LUZ19 phages

\section{Discussion}

Current phage therapy protocols for treating infections in orthopedics is limited to repeated administration of bacteriophages through external draining systems for a duration of up to 10 days [11]. Such draining systems can be considered a burden for the patient and pose substantial risk for 
bacterial contamination, potentially leading to an additional hospital acquired infection. In this paper we investigated the potential of a thermoresponsive HA-pNIPAM hydrogel and alginate microbead materials for bacteriophage delivery through sustained release of embedded bacteriophages, with the aim to allow primary wound closure and avoid the need for any percutaneous draining system for local phage therapy.

Embedding bacteriophages in biomaterials comes with the risk of phage inactivation. Inactivation of bacteriophages can occur when the protein structure of the phage is altered due to exposure to heat, mechanical stresses, or chemical biocides [12]. When phages are embedded in a biomaterial under ambient conditions (i.e. mild mixing and at low temperatures), its chemical environment was recognized to be the main cause of potential phage inactivation in this study. Also, thermoresponsive HA-pNIPAM undergoes physical crosslinking at physiological temperatures, making it worthwhile to study phage activity in this phase-transitioning environment. As tailed phages such as ISP are reported to be susceptible to structural damage due to mechanical stresses [13], it was a positive outcome to see the any mechanical stress involved in gelation and hydrogel preparation did not cause significant phage inactivation.

Hydrogels are not the only biomaterial class for therapeutic delivery to bone. Drug (or phage) delivery to the site of bone infection could also be realized through targeted delivery with micro- or nanoparticles [14].While literature on particulate phage delivery systems for bone was not found, phage delivery to the respiratory system has been described in poly(lactide-co-glycolic acid) (PLGA) microparticles by Agarwal et al. (2018) [15]. Phages were adsorbed on the particles after particle fabrication to limit phage exposure to harmful 
environmental cues. As phage release was limited to endpoint analysis, no information on the release kinetics of phage from PLGA microparticles is known. However, with in vivo treatments assessed after 24 hours, release of phage is expected to be burst-like. This makes our hydrogel approach, facilitating sustained release over 21 days, a preferred method for phage delivery for orthopedic infections.

Alginate and chitosan are established biomaterials for bacteriophage delivery with abundant literature available [6,8,16-18]. The release of phage from alginate materials is diffusion driven and expedited by swelling and subsequent disintegration of the alginate material. Alginate microbeads are kept together in aqueous media by interactions with $\mathrm{Ca}^{2+}$ ions with guluronate segments of alginate, but structural integrity is affected by ion exchange of $\mathrm{Ca}^{2+}$ with other monovalent ions present such as $\mathrm{Na}^{+}[19]$. The swelling and degradation behavior of alginate was affected by $\mathrm{NaCl}$ concentration with increased swelling rates observed at higher $\mathrm{NaCl}$ concentrations [20]. Alternatively, alginate microbeads did not show swelling in distilled water, indicating that ion exchange is the main contributor to alginate bead swelling and subsequent degradation [20]. Bajpai et al. (2004) showed that alginate microbeads (with an unknown diameter) took approximately 4-5 hours to disintegrate in PBS [20], however no rapid alginate degradation was observed for our alginate or alginate-chitosan microbeads. In our setup, SM-buffer was used to ensure optimal phage viability upon release from the microbeads. SM-buffer contains $100 \mathrm{mM}$ $\mathrm{NaCl}(5.8 \mathrm{~g} / \mathrm{L})$, similar to the $\mathrm{NaCl}$ concentration in PBS $(8 \mathrm{~g} / \mathrm{L}, 137 \mathrm{mM})$ commonly used in physiologically relevant in vitro studies. Additionally, SMbuffer contained $8.3 \mathrm{mM} \mathrm{MgSO} 4(1.0 \mathrm{~g} / \mathrm{L}) . \mathrm{Mg}^{2+}$ ions have been identified as ions that induce alginate gelation, however a 5-10 times higher $\mathrm{Mg}^{2+}$ ion 
concentration was necessary to yield similar results compared to $\mathrm{Ca}^{2+}$ gelation [21]. As the alginate and alginate-chitosan beads in our study where already gelled in $\mathrm{CaCl}_{2}$ solution prior to exposure to SM-buffer, we cannot exclude that the $\mathrm{Mg}^{2+}$ ions in SM-buffer contributes to the reduced microbead degradation.

As most bacteriophages have an isoelectric point between 3 and 7 [22], the cationic character of chitosan was expected to slow bacteriophage release from alginate-chitosan microbeads in $\mathrm{pH}$ neutral buffers. The effects of the microbeads chitosan coating on phage release was most profound for LUZ19 phages in combined HA-pNIPAM + alginate-chitosan materials, where a reduced release was observed over the first 9 days of the release experiment (Figure 6D). This finding is supported by literature on short-term release of T4 phages from alginate-chitosan materials [18]. However, LUZ19 release from HA-pNIPAM + alginate-chitosan materials increased significantly compared to HA-pNIPAM and HA-pNIPAM + alginate materials after 9 days. If we consider that the alginate and alginate-chitosan microbeads continually lose their structural integrity after approximately 9 days in SM-buffer, the higher release of LUZ19 from alginate-chitosan microbeads could be established due to higher maintenance of phage activity in alginate-chitosan microbeads. There is abundant literature available that reports on the protective properties of chitosan coatings for embedded bacteriophages that supports the commentary above [16-18].

As mentioned before, striking differences were found between ISP phage and LUZ19 phage release from alginate and alginate-chitosan microbeads (Figure 7.5), which were again observed when the microbeads were embedded in HA-pNIPAM hydrogels (Figure 7.6). Bacteriophage mobility 
inside a hydrogel is affected by the hydrophobicity of the bacteriophage proteome [23]. Vandersteegen et al. (2011) showed that ISP phage proteome consisted of 211 proteins, with only a hand full of these proteins making up the majority of the phage protein mass [24]. Upon consulting the NCBI gene databank it was found that $39 \%$ of amino acids from these proteins consisted of hydrophobic amino acids (Gly, Ala, Val, Leu, Ile, Pro, Phe, Met or Trp). The LUZ19 genome was published by Lammens et al. (2009) [25] and the hydrophobic amino acid content in the most abundant proteins was 59\%. This increased hydrophobic properties of LUZ19 compared to ISP with hydrophobic pNIPAM aggregations in gelled HA-pNIPAM can explain the differences in release kinetics observed in Figure 6.5 and Figure 6.6 and the higher LUZ19 retention in HA-pNIPAM gels after 21 days.

\section{Conclusion}

Thermoresponsive HA-pNIPAM hydrogels and alginate-chitosan microbeads were investigated as phage delivery materials. HA-pNIPAM hydrogels show sustained release of ISP and LUZ19 phages for 21 days. Although being a phage delivery material with easy handling and efficient phage incorporation, HA-pNIPAM hydrogels show moderate anti-viral effects over prolonged incubation times, highlighting the advantages of coformulations with alginate materials for improved phage activity. Alginatechitosan microbeads in combination with HA-pNIPAM hydrogels showed the most constant and sustained release of LUZ19 bacteriophages over a period of 21 days. These finding suggests that release kinetics are phage dependent and utilizing these delivery systems for other phages requires extensive evaluation. The investigated hydrogel systems show potential for long term bacteriophage release and further in vitro and in vivo experiments 
should show the efficacy of these phage delivery systems in preventing and treating internal infections.

\section{Acknowledgements}

The authors would like to thank Flavio Linardi for his performance of the ${ }^{1} \mathrm{H}-\mathrm{NMR}$ measurements and assistance in the HA-pNIPAM synthesis. This work has been funded by the JPIAMR: Cross border research program, Grant: 40AR40_185917

\section{References}

1 Kortright, K. E., Chan, B. K., Koff, J. L. \& Turner, P. E. Phage Therapy: A Renewed Approach to Combat Antibiotic-Resistant Bacteria. Cell Host \& Microbe 25, 219-232, doi:10.1016/j.chom.2019.01.014 (2019).

2 Carlton, R. M. Phage therapy: past history and future prospects. Arch Immunol Ther Exp (Warsz) 47, 267-274 (1999).

3 Abedon, S. T. in Viruses: Essential Agents of Life (ed Günther Witzany) 389-405 (Springer Netherlands, 2012).

4 Peroglio, M., Eglin, D., Benneker, L. M., Alini, M. \& Grad, S. Thermoreversible hyaluronan-based hydrogel supports in\&\#xa0;vitro and ex\&\#xa0;vivo disc-like differentiation of human mesenchymal stem cells. The Spine Journal 13, 1627-1639, doi:10.1016/j.spinee.2013.05.029 (2013).

5 ter Boo, G.-J. A. et al. Injectable gentamicin-loaded thermo-responsive hyaluronic acid derivative prevents infection in a rabbit model. Acta Biomaterialia 43, 185-194, doi:https://doi.org/10.1016/j.actbio.2016.07.029 (2016).

6 Alves, D. et al. Bacteriophage $\phi I B B-P F 7 A$ loaded on sodium alginate-based films to prevent microbial meat spoilage. International Journal of Food Microbiology 291, 121 127, doi:https://doi.org/10.1016/j.ijfoodmicro.2018.11.026 (2019).

7 Colom, J. et al. Microencapsulation with alginate/CaCO3: A strategy for improved phage therapy. Scientific Reports 7, 41441, doi:10.1038/srep41441 (2017).

8 Kim, S., Jo, A. \& Ahn, J. Application of chitosan-alginate microspheres for the sustained release of bacteriophage in simulated gastrointestinal conditions. International Journal of Food Science \& Technology 50, 913-918, doi:10.1111/ijfs.12736 (2015).

9 D'Este, M., Alini, M. \& Eglin, D. Single step synthesis and characterization of thermoresponsive hyaluronan hydrogels. Carbobydrate polymers 90, 1378-1385, doi:10.1016/j.carbpol.2012.07.007 (2012).

10 Choińska-Pulit, A., Mituła, P., Śliwka, P., Łaba, W. \& Skaradzińska, A. Bacteriophage encapsulation: Trends and potential applications. Trends in Food Science \& Technology 45, 212-221, doi:https://doi.org/10.1016/j.tifs.2015.07.001 (2015). 
11 Onsea, J. et al. Bacteriophage therapy as a treatment strategy for orthopaedicdevice-related infections: where do we stand? European cells \& materials 39, 193-210, doi:10.22203/eCM.v039a13 (2020).

12 Guglielmotti, D. M., Mercanti, D. J., Reinheimer, J. A. \& Quiberoni, A. D. L. Review: efficiency of physical and chemical treatments on the inactivation of dairy bacteriophages. Front Microbiol 2, 282-282, doi:10.3389/fmicb.2011.00282 (2012).

13 Leung, S. S. Y. et al. Jet nebulization of bacteriophages with different tail morphologies - Structural effects. International Journal of Pharmacentics 554, 322-326, doi:https://doi.org/10.1016/i.ijpharm.2018.11.026 (2019).

14 Rotman, S. G. et al. Development of bone seeker-functionalised microspheres as a targeted local antibiotic delivery system for bone infections. Journal of Orthopaedic Translation, doi:https://doi.org/10.1016/j.jot.2019.07.006 (2019).

15 Agarwal, R. et al. Inhaled bacteriophage-loaded polymeric microparticles ameliorate acute lung infections. Nature Biomedical Engineering 2, 841-849, doi:10.1038/s41551-018-0263-5 (2018).

16 Abdelsattar, A. S., Abdelrahman, F., Dawoud, A., Connerton, I. F. \& El-Shibiny, A. Encapsulation of E. coli phage ZCEC5 in chitosan-alginate beads as a delivery system in phage therapy. AMB Express 9, doi:10.1186/s13568-019-0810-9 (2019). $\mathrm{Ma}, \mathrm{Y}$. et al. Microencapsulation of bacteriophage felix O1 into chitosan-alginate microspheres for oral delivery. Appl Environ Microbiol 74, 4799-4805, doi:10.1128/AEM.00246-08 (2008).

Moghtader, F., Eğri, S. \& Piskin, E. Phages in modified alginate beads. Artificial Cells, Nanomedicine, and Biotechnology 45, 357-363, doi:10.3109/21691401.2016.1153485 (2017).

19 Lee, K. Y. \& Mooney, D. J. Alginate: properties and biomedical applications. Prog Polym Sci 37, 106-126, doi:10.1016/j.progpolymsci.2011.06.003 (2012).

20 Bajpai, S. K. \& Sharma, S. Investigation of swelling/degradation behaviour of alginate beads crosslinked with $\mathrm{Ca} 2+$ and $\mathrm{Ba} 2+$ ions. Reactive and Functional Polymers 59, 129-140, doi:https://doi.org/10.1016/j.reactfunctpolym.2004.01.002 (2004).

21 Topuz, F., Henke, A., Richtering, W. \& Groll, J. Magnesium ions and alginate do form hydrogels: a rheological study. Soft Matter 8, 4877-4881, doi:10.1039/C2SM07465F (2012).

22 Michen, B. \& Graule, T. Isoelectric point of viruses. Journal of applied microbiology 109, 388-397, doi:10.1111/j.1365-2672.2010.04663.x (2010).

23 Sautrey, G., Brié, A., Gantzer, C. \& Walcarius, A. MS2 and Q $\beta$ bacteriophages reveal the contribution of surface hydrophobicity on the mobility of nonenveloped icosahedral viruses in SDS-based capillary zone electrophoresis. ELECTROPHORESIS 39, 377-385, doi:10.1002/elps.201700352 (2018).

24 Vandersteegen, K. et al. Microbiological and molecular assessment of bacteriophage ISP for the control of Staphylococcus aureus. PLoS One 6, e24418e24418, doi:10.1371/journal.pone.0024418 (2011).

25 Lammens, E. et al. Representational Difference Analysis (RDA) of bacteriophage genomes. Journal of Microbiological Methods 77, 207-213, doi:https://doi.org/10.1016/j.mimet.2009.02.006 (2009). 


\section{Chapter 8}

\section{Synergistic anti-fouling and bactericidal poly(ether ether ketone) surfaces via a one-step photomodification}

Sytze Buwalda ${ }^{1}$, Stijn Rotman ${ }^{2,3}$, David Eglin ${ }^{2,3}$, Fintan Moriarty ${ }^{2}$, Audrey Bethry ${ }^{1}$, Xavier Garric ${ }^{1}$, Olivier Guillaume ${ }^{2}$ and Benjamin Nottelet ${ }^{1}$

${ }^{1}$ IBMM, University of Montpellier, CNRS, ENSCM, Montpellier, France

${ }^{2}$ AO Research Institute Davos, Davos Platz, Switzerland

${ }^{3}$ Department of Biomaterials Science and Technology, Faculty of Science and Technology, University of Twente, Enschede, The Netherlands

‡ These authors contributed equally to the manuscript. 


\section{Abstract}

Implants of poly(ether ether ketone) (PEEK) are gaining importance in surgical bone reconstruction of the skull. As with any implant material, PEEK is susceptible to bacterial contamination and occasionally PEEK implants were removed from patients because of infection. To address this problem, a combination of anti-fouling and bactericidal polymers are grafted onto PEEK. The originality is that anti-fouling (modified poly(ethylene glycol)) and bactericidal (quaternized poly(dimethylaminoethyl acrylate)) moieties are simultaneously and covalently grafted onto PEEK via UV photoinsertion. The functionalized PEEK surfaces are evaluated by water contact angle measurements, FTIR, XPS and AFM. Grafting of anti-fouling and bactericidal polymers significantly reduces Staphylococcus aureus adhesion on PEEK surfaces without exhibiting cytotoxicity in vitro. This study demonstrates that grafting combinations of anti-fouling and bactericidal polymers synergistically prevents bacterial adhesion on PEEK implants. This approach shows clinical relevance as grafting is rapid, does not modify PEEK properties and can be conducted on pre-formed implants.

\section{Introduction}

The introduction of poly(ether ether ketone) (PEEK), almost two decades ago, had a tremendous impact in orthopedics [1,2]. Due to its excellent biocompatibility, radiolucency, inertness, strength and processability, PEEK based implants are overtaking many other materials such as titanium alloys [3]. Importantly, PEEK prostheses can easily be manufactured with modern 3D printing technology, using data originating from the patient's computed tomography scan. As a consequence, the fabrication of customized PEEK patient implants for craniomaxillo-facial reconstruction has been recently 
reported with promising results in terms of aesthetics and biological functionality [4-6]. Such patient specific implants made of PEEK constitute an excellent option for the reconstruction of large and complex defects and represent an emerging alternative for titanium implants. Due to the aforementioned advantages, the use of PEEK implants is currently spreading to other surgical fields, including spinal fusion (PEEK PREVAIL ${ }^{\mathrm{TM}}$ from Medtronic), [7] stabilization of osteotomy (iBalance ${ }^{\circledR}$ HTO implants and anchors from Arthrex), [8] intra-medullar nails (composite carbon-PEEK biomaterial Carbofix from Orthopedics) [9] or dental implants (commercialized by IQ Implant) [10] to mention only some of them.

Unfortunately, contamination of PEEK implants by bacteria is still a recurrent complication and source of printed implant failure, even upon administration of local and systemic anti-bacterial therapy [4-6]. For this reason, several researchers have focused their attention on the chemical modification of the surface of PEEK implants to endow them with antiinfective properties. Two main strategies have been used to prevent implantrelated infection: (1) minimizing the bacterial attachment or (2) killing bacteria present on the surface of the implant. In the first category, Ishihari et al. grafted a coating of poly(2-methacryloyloxyethyl phosphorylcholine) (PMPC), a highly hydrophilic polymer, onto the surface of PEEK via photoinduced graft polymerization of MPC[11] or via aryl-azide UV photoinsertion of PMPC.[12] It was found that the increased surface wettability of PEEK-PMPC $\left(<10^{\circ}\right.$ for PEEK-PMPC compared to $90^{\circ}$ for untreated PEEK) offered a certain degree of protection in vitro against E. coli adhesion [13]. PEEK has also been endowed with anti-fouling properties by UV irradiation-assisted grafting of poly(octafluoropentyl methacrylate) [14] and poly(oligo(ethylene glycol) methacrylate) [15]. However, the efficacy of 
these 'passive' anti-fouling coatings on the surface of medical devices can be questioned, as it generally only decreases partially and temporarily the number of bacteria attaching to the surface of the implants. Consequently, several research teams have conferred 'active' bactericidal protection to PEEK, for example by coating the surface with silver containing formulations [16] or by mixing PEEK granules with silver before implant manufacture [17]. However, the use of silver-based medical devices has several disadvantages, including the development of resistance by bacteria, [18] possible leakage of silver nanoparticles at toxic levels, $[19,20]$ as well as increased surface roughness, which may favor bacterial adhesion [21]. Furthermore, over the course of exposure and due to the accumulation of bacterial debris, the bactericidal groups can be masked and become ineffective against the subsequent bacteria colonizing the implants [22]. In this respect, conferring dual-functionality by grafting a combination of an anti-fouling polymer and a bactericidal agent could be a potent combination to prevent PEEK implant infection. In order to have a clinical impact, the grafting of those moieties must be feasible onto the surface of existing preformed implants, must be rapid and should not alter the physico-chemical and biological properties of the PEEK. Grafting should also be feasible without pre-functionalization or pre-activation steps.

In this respect, the use of aryl-azide groups is an attractive approach, [23] as they are converted into reactive nitrene intermediates upon UV irradiation that can form covalent bonds by insertion into carbon-hydrogen bonds which are present in most polymer substrates. This prompted us to explore a new strategy for the prevention of PEEK implant infection by grafting a combination of anti-fouling polymers as well as bactericidal polymers onto PEEK using aryl-azide UV photoinsertion. 
In this manuscript, we first describe the synthesis and characterization of aryl-azide containing anti-fouling poly(ethylene glycol) (PEG) and bactericidal quaternized poly(dimethylaminoethyl acrylate) (P(qDMAEMA)) polymers. Subsequently, we discuss their covalent grafting onto PEEK via UV photoinsertion. Lastly, we demonstrate the anti-fouling and bactericidal potential of the modified PEEK surfaces during in vitro exposure to planktonic bacteria and validate the absence of leachable toxic compounds from the final biomaterials on fibroblasts. To the best of our knowledge, this is the first report describing a one-step chemical functionalization of PEEK with synergistically operating anti-fouling and bactericidal polymers. This chemical approach is of great interest as it can potentially be applied not only to PEEK, but also to the vast majority of other polymer-based implants as it requires only substrates with available carbon-hydrogen bounds.

\section{Experimental section}

\section{Materials}

4-Azidobenzoic acid was purchased from TCI Europe (Zwijndrecht, The Netherlands). 4-Aminophenol hydrochloride, sodium nitrite, sodium azide, 2-cyano-2-propyl benzodithioate (CPDB), 2,2'-azobis(2-methylpropionitrile) (AIBN), 2-(dimethylamino)ethyl methacrylate (DMAEMA), 1-iododecane, DL-1-amino-2-propanol, methacryloyl chloride, phosphate buffered saline (PBS), 4-(dimethylamino)pyridine (DMAP), p-toluenesulfonic acid, triethylamine (TEA), 4,4'-azobis(4-cyanopentanoic acid) (ABCPA), poly(ethylene glycol) methyl ether $\left(M_{n}=2.000,5.000\right.$ and $12.000 \mathrm{~g} \cdot \mathrm{mol}^{-1}$, mPEG2K, mPEG5K and mPEG12K, respectively) and dicyclohexylcarbodiimide (DCC) were all obtained from Sigma-Aldrich (StQuentin Fallavier, France). 4-Azidophenol,[24] 2-hydroxypropyl 
methacrylamide (HPMA)[25] and 4-(dimethylamino)pyridinium-4toluenesulfonate (DPTS)[26] were synthesized as reported previously. Dichloromethane (DCM) and TEA were dried over calcium hydride and potassium hydroxide, respectively, and distilled prior to use. Tryptic soy agar (TSA) plates were purchased from Oxoid (Wesel, Germany) and were checked for sterility by overnight incubation at $37^{\circ} \mathrm{C}$. Tryptic soy broth (TSB) was bought from Oxoid (Basingstoke Hampshire, England). 2Propanol was purchased from Roth (Karlsruhe, Germany). Two bacterial strains were used in this study, Staphylococcus aureus (S. aureus, NCTC 12973) and Escherichia coli (E. coli, NCTC 12441). LIVE/DEAD ${ }^{\circledR}$ BacLight $^{\mathrm{TM}}$ Bacterial Viability Kit L13152 was purchased from Molecular Probes (Leiden, the Netherlands). Pooled human serum from two donors (non-heat inactivated) was collected and kindly donated by the Blutspendedienst SRK Graubünden.

\section{Synthesis}

\section{4-azidophenyl methacrylate}

4-Azidophenyl methacrylate (4-APM) was synthesized following a previously reported procedure [27]. Detailed synthetic procedure and ${ }^{1} \mathrm{H}$ NMR peaks assignments are provided in the supplementary data.

\section{(PEG-XK)-P(HPMA-N 3 ) diblock copolymers}

(PEG-XK)-P(HPMA-N 3 ) diblock copolymers were synthesized in 3 steps. $(\mathrm{PEG}-2 \mathrm{~K})_{2}$-ABCPA, (PEG-5K) ${ }_{2}$-ABCPA and (PEG-12K $)_{2}$-ABCPA macro-initiators were first synthesized starting from mPEG2K, mPEG5K and $\mathrm{mPEG} 12 \mathrm{~K}$, respectively according to a procedure previously reported 
[28]. The detailed synthetic procedure and ${ }^{1} \mathrm{H}$ NMR peaks assignments are provided in the supplementary data.

SEC: $M_{n} 3800 \mathrm{~g} \mathrm{~mol}^{-1}, \oslash 1.34\left((\mathrm{PEG}-2 \mathrm{~K})_{2}-\mathrm{ABCPA}\right) ; M_{n} 12700 \mathrm{~g} \mathrm{~mol}^{-1}, \oslash$ 1.17 ((PEG-5K) $\left.{ }_{2}-\mathrm{ABCPA}\right)$; SEC: $M_{n} 20800 \mathrm{~g} \mathrm{~mol}^{-1}$, D 1.24 ((PEG-12K) $)_{2-}^{-}$ ABCPA).

(PEG-2K)-P(HPMA), (PEG-5K)-P(HPMA) and (PEG-12K)-P(HPMA) diblock copolymers were then synthesized starting from (PEG-2K) ${ }_{2}$ ABCPA, (PEG-5K) ${ }_{2}-\mathrm{ABCPA}$ and (PEG-12K) ${ }_{2}-\mathrm{ABCPA}$, respectively.[28] The detailed synthetic procedure and ${ }^{1} \mathrm{H}$ NMR peaks assignments are provided in the supplementary data.

SEC: $M_{n} 4900 \mathrm{~g} \mathrm{~mol}^{-1}, Ð 1.24$ ((PEG-2K)-P(HPMA)); $M_{n} 8800 \mathrm{~g} \mathrm{~mol}^{-1}, Ð$ 1.34 ((PEG-5K)-P(HPMA)); $M_{n} 22600 \mathrm{~g} \mathrm{~mol}^{-1}, Ð 1.07$ ((PEG-12K)$\mathrm{P}(\mathrm{HPMA}))$.

Finally, (PEG-2K)-P(HPMA-N 3 ), (PEG-5K)-P(HPMA-N ${ }_{3}$ ) and (PEG$12 \mathrm{~K})-\mathrm{P}\left(\mathrm{HPMA}-\mathrm{N}_{3}\right)$ diblock copolymers were synthesized starting from (PEG-2K)-P(HPMA), (PEG-5K)-P(HPMA) and (PEG-12K)-P(HPMA), respectively by esterification of $\mathrm{P}(\mathrm{HPMA})$ units with 4-azidobenzoic acid using DMAP as catalyst and dry DMF as solvent. The details of this last synthetic procedure are provided in the supplementary data. The typical ${ }^{1} \mathrm{H}$ NMR peaks assignments of this new family of copolymers are provided below.

${ }^{1} \mathrm{H}$ NMR (300 MHz, DMSO-d 6 , 8): 7.96 (br, 38H, phenyl protons), 7.21 (br, $38 \mathrm{H}$, phenyl protons), 5.00 (br, $\left.19 \mathrm{H}, \mathrm{NH}-\mathrm{CH}_{2}-\mathrm{CH}\left(\mathrm{CH}_{3}\right)-\mathrm{O}-\mathrm{C}=\mathrm{O}\right), 4.72$ (br, $\left.19 \mathrm{H}, \mathrm{CH}\left(\mathrm{CH}_{3}\right)-\mathrm{OH}\right), 3.51$ (m, 510H, PEG protons), 3.24 (s, $3 \mathrm{H}$, $\mathrm{CH}_{3}-\mathrm{O}-\mathrm{CH}_{2}-\mathrm{CH}_{2}$ ), 2.90 (br, 76H, NH-CH$-\mathrm{CH}$ ), 1.4 - 2.0 (br, 76H, 
backbone methylene protons), 0.99 (br, $\left.114 \mathrm{H}, \mathrm{NH}-\mathrm{CH}_{2}-\mathrm{CH}\left(\mathrm{CH}_{3}\right)-\mathrm{O}\right), 0.7$ 0.9 (br, 114H, backbone methyl protons).

\section{$\underline{\text { P(DMAEMA-co-APM) copolymer }}$}

AIBN (13 mg, 7.6.10-2 mmol), CPDB (55 mg, $0.25 \mathrm{mmol}$ ), 4-APM (0.5 g, $2.5 \mathrm{mmol})$ and DMAEMA (0.83 ml, $4.9 \mathrm{mmol})$ were dissolved in $10 \mathrm{ml}$ of dioxane. The solution was stirred at $70{ }^{\circ} \mathrm{C}$ overnight under reflux in an argon atmosphere. The product was subsequently precipitated in cold heptane. P(DMAEMA-co-APM) was obtained by filtration and dried overnight in vacuo to give orange crystals. Yield: $0.42 \mathrm{~g}(33 \%)$. ${ }^{1} \mathrm{H}$ NMR $(300 \mathrm{MHz}$, $\mathrm{CDCl}_{3}, \delta$ ): $6.7-7.2$ (br, $16 \mathrm{H}$, phenyl protons), $4.0-4.2$ (br, $16 \mathrm{H}, \mathrm{O}-\mathrm{CH}_{2-}$ $\left.\mathrm{CH}_{2}-\mathrm{N}\right), 2.5-2.7$ (br, $\left.16 \mathrm{H}, \mathrm{O}_{-} \mathrm{CH}_{2}-\mathrm{CH}_{2}-\mathrm{N}\right), 2.1-2.4$ (br, 48H, N-(CH$)_{2}$ ), 0.8 - 2.1 (60H, backbone methyl and methylene protons). SEC: $M_{n} 2200 \mathrm{~g}$ $\mathrm{mol}^{-1}, \oplus 2.7$.

\section{Quaternization of P(DMAEMA-co-APM)}

P(DMAEMA-co-APM) (0.3 g, $0.14 \mathrm{mmol})$ and 1-iododecane $(2.5 \mathrm{ml}, 12$ mmol) were dissolved in $3 \mathrm{ml}$ dioxane. The solution was stirred at $70{ }^{\circ} \mathrm{C}$ overnight under reflux in an argon atmosphere. The product was dissolved in DCM and precipitated in cold heptane. P(qDMAEMA-co-APM) was obtained by filtration and dried overnight in vacuo to a give a light brown powder. Yield: $0.48 \mathrm{~g}(80 \%)$.

\section{Characterization of monomer and polymers}

${ }^{1} \mathrm{H}$ NMR spectra were recorded on a Bruker AMX300 spectrometer at 25 ${ }^{\circ} \mathrm{C}$. Compounds were dissolved in $\mathrm{CDCl}_{3}, \mathrm{DMSO}_{-} \mathrm{d}_{6}$ or $\mathrm{D}_{2} \mathrm{O}$ at a concentration of $15 \mathrm{mg} \mathrm{ml}^{-1}$. As a standard, residual internal $\mathrm{CHCl}_{3}(\delta 7.26)$, DMSO $(\delta 2.50)$ or $\mathrm{H}_{2} \mathrm{O}(\delta 4.79)$ was used. 
The number-average and weight-average molar masses $\left(M_{n}\right.$ and $M_{w}$, respectively) and dispersity $\left(\nexists, M_{w} / M_{n}\right)$ of the polymers were determined by size exclusion chromatography (SEC). For the (PEG-XK) ${ }_{2}-\mathrm{ABCPA}$ and (PEG-XK)-P(HPMA) polymers, a Viscotek GPCmax VE2100 liquid chromatograph equipped with a Viscotek VE3580 refractive index detector operating at $35{ }^{\circ} \mathrm{C}$ was employed. Tetrahydrofuran was used as the eluent and the flow rate was set up at $1.0 \mathrm{ml} \mathrm{min}^{-1}$. Two LT5000L $300 \times 7.8 \mathrm{~mm}$ columns operating at $29{ }^{\circ} \mathrm{C}$ were used. Calibrations were performed with polystyrene standards. For the P(DMAEMA-co-APM) polymer, measurements were performed on a system consisting of a Waters 515 HPLC pump and a Waters 410 refractive index detector operating at $40^{\circ} \mathrm{C}$. Dimethylformamide with $0.1 \% \mathrm{LiBr}$ was used as the eluent and the flow rate was set up at $1.0 \mathrm{ml} \mathrm{min}{ }^{-1}$. One Polargel M precolumn $(50 \times 7.5 \mathrm{~mm})$ and two Polargel $\mathrm{M}$ columns $(300 \times 7.5 \mathrm{~mm})$ operating at $60{ }^{\circ} \mathrm{C}$ were used. Calibrations were performed with poly(methyl methacrylate) standards.

\section{Preparation of functionalized PEEK surfaces}

Medical grade PEEK (PEEK-OPTIMA ${ }^{\text {TM }}$, Invibio Ltd, UK) discs of $13 \mathrm{~mm}$ in diameter and $2 \mathrm{~mm}$ in height were used. Anti-fouling polymer (PEG$\mathrm{XK})-\mathrm{P}\left(\mathrm{HPMA}_{3}\right)$ ), bactericidal polymer (P(qDMAEMA-co-APM)) or a combination of both $(1 / 1 \mathrm{w} / \mathrm{w})$ was dissolved in ethanol at a concentration of $5 \mathrm{mg} \mathrm{ml}^{-1}$. After filtration through $0.45 \mathrm{~mm}$ syringe filters, the solution was applied on the surface of PEEK discs using an Infinity airbrush. After evaporation of the ethanol, the PEEK discs were irradiated for $30 \mathrm{~s}$ using a Dymax PC-1200 UV curing flood lamp (mercury lamp, 400 W). After irradiation the surfaces were washed in ethanol for 10 min using an ultrasonic 
bath, followed by drying in vacuo. The surface functionalization procedure was repeated twice.

\section{Characterization of functionalized PEEK surfaces}

The functionalized PEEK surfaces were characterized by Fourier Transform Infrared Spectroscopy (FTIR) analyses, X-ray photoelectron spectroscopy (XPS) analyses, and Atomic Force Microscopy (AFM) analyses. Static contact angle measurements were also performed. Details of equipment and procedures are provided as supplementary data.

\section{In vitro assessment of anti-fouling properties}

Following the surface modification of the complete PEEK surface and prior to biological testing, the discs were cleaned by sonication in 2-propanol (15 min at $400 \mathrm{~W}), 70 v / v \%$ ethanol $(10 \mathrm{~min}$ at $400 \mathrm{~W})$ and $\mathrm{dH}_{2} \mathrm{O}(10 \mathrm{~min}$ at $400 \mathrm{~W}$ ) in a sonication bath (Sonorex Bandelin, Berlin, Germany). The PEEK discs were allowed to dry under sterile laminar flow overnight. PEEK discs grafted with (PEG-XK)-P(HPMA-N $\left.{ }_{3}\right)(\mathrm{n}=3)$ were incubated using standard culture condition $\left(37^{\circ} \mathrm{C}\right.$ and $\left.5 \% \mathrm{CO}_{2}\right)$ for $1 \mathrm{~h}$ in $3 \mathrm{ml}$ of $S$. aureus or E. coli suspension in PBS (calibrated to an $O D_{600}$ of 0.05 , corresponding to approximately $3.0 \times 10^{7}$ colony forming units $(\mathrm{CFU}) \mathrm{ml}^{-1}$ ) and kept under mild agitation using a shaker $(30 \mathrm{rpm})$. After $1 \mathrm{~h}$, the discs were removed from the bacterial suspension, washed three times in fresh solutions of PBS, and subsequently sonicated in a sonication bath at $400 \mathrm{~W}$ for $5 \mathrm{~min}$ in $5 \mathrm{ml}$ of PBS to remove adherent bacteria from the PEEK discs. The sonicates were serial diluted in PBS $(1 / 10 v / v)$ and $20 \mu \mathrm{l}(\mathrm{n}=6)$ of each dilution was plated on TSA plates. CFU were counted manually on the agar plates after $18 \mathrm{~h}$ of incubation $\left(37^{\circ} \mathrm{C}\right.$ and $\left.5 \% \mathrm{CO}_{2}\right)$. 


\section{In vitro assessment of combined anti-fouling and bactericidal properties}

PEEK discs grafted with (PEG-XK)-P(HPMA-N 3 ) and/or P(qDMAEMAco-APM) were cleaned by sonication in alcohol solutions as described previously. The discs were incubated for $1 \mathrm{~h}$ or for $24 \mathrm{~h}$ in $3 \mathrm{ml}$ of $S$. aureus suspension in PBS $\left(O D_{600}=0.05\right)$. In this experiment, the bacteria suspensions were supplemented with $2 \% v / v$ human serum and $2 \% v / v$ TSB to simulate semi-physiological conditions. After $1 \mathrm{~h}$ or $24 \mathrm{~h}$ incubation under shaking (30 rpm), the discs were washed 3 times by transferring them to fresh solutions of PBS. The remaining adherent bacteria were sonicated, plated and counted as described previously. For every investigated PEEK disc, a sonicate bacterial dispersion was stained with a LIVE/DEAD ${ }^{\circledR}$ BacLight ${ }^{\mathrm{TM}}$ Bacterial Viability Kit L13152 according to manufacturer's instructions and subsequently imaged by a ZEISS LSM 800 confocal microscope (Carl Zeiss, Feldbach, Switzerland).

\section{Stability of coating during alcohol cleaning versus steam sterilization}

Alcohol cleaning of PEEK discs was done in three steps as described previously. Steam sterilization of PEEK discs occurred at standard conditions of $121{ }^{\circ} \mathrm{C}$ for $20 \mathrm{~min}$. PEEK discs ( $\mathrm{n}=3$ ) cleaned/sterilized by one of both methods were exposed to $S$. aureus suspension in PBS as described earlier.

\section{Evaluation of cytotoxicity via extraction method (ISO 10993-5)}

A detailed procedure concerning the evaluation of the cytotoxicity of the coatings is provided in the supplementary data. 


\section{Murine subcutaneous infection model}

PEEK implants (diameter $=5 \mathrm{~mm}$, height $=2 \mathrm{~mm}$ ) were subcutaneously implanted on the lower backs of anesthetized BALB/c mice. Every mouse bore two subdermal implants, one on the right side of the spine and one on the left side. After implantation of the PEEK implant, $10 \mu \mathrm{L}$ of bacterial suspension $\left(3.6 \cdot 10^{8} \mathrm{CFU} / \mathrm{mL}\right)$ was pipetted on top of the implant and the incisions were subsequently closed with sutures. After 24 hours, the mice were euthanized by means of cervical dislocation and the implants and surrounding soft tissues were harvested. The PEEK implants were washed in PBS and subsequently sonicated in PBS for 10 minutes in glass vials and the soft tissues were homogenized. The rinsing PBS, homogenized soft tissues and the implant sonication buffer were serial diluted and $200 \mu \mathrm{L}$ was spread on agar plates.

The experiments were approved by the Cantonal Ethics Committee (TVB number : 18_2018)

\section{Results and discussion}

\section{Synthesis and characterization of anti-fouling and bactericidal polymers}

PEEK exhibits unprecedented advantages compared to commonly used materials in implantology, with one of the most attractive ones being its processability by additive manufacturing [4]. Nevertheless, as with any other implant material, PEEK is not free of bacterial contamination $[6,29,30]$. We covalently grafted a combination of anti-fouling and bactericidal polymers on the PEEK surface as a potentially synergistic, powerful combination to prevent PEEK implant infection (Figure 8.1). 


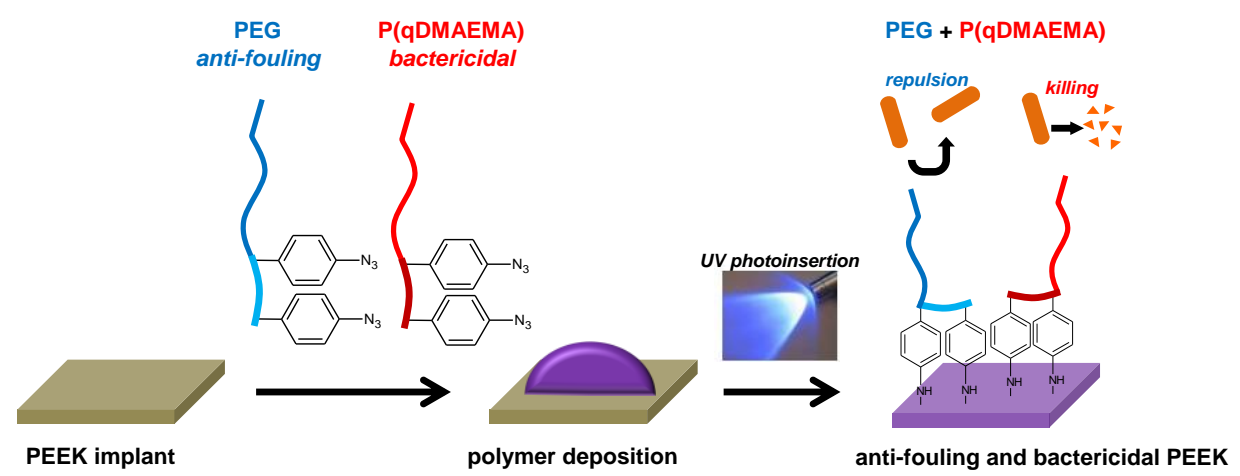

Figure 8.1 - Schematic illustration of the grafting process of synergistically operating anti-fouling PEG and bactericidal P(qDMAEMA) onto PEEK via aryl-azide UV photoinsertion.

As anti-fouling polymer we selected PEG, as its immobilization onto a biomaterial surface has been shown to increase hydrophilicity and decrease protein adsorption, thereby preventing cells to attach [31]. The PEG block was extended with a second polymer block, poly(2-hydroxypropyl methacrylamide) (P(HPMA)), to allow for subsequent P(HPMA) functionalization with aryl-azide groups (Figure 8.2), which facilitate grafting onto the PEEK surface via UV photoinsertion. We chose P(HPMA) as the second polymer block because of its hydrophilicity, biocompatibility, nonimmunogenicity and the possibility for straightforward chemical modification via derivatization of its pendant hydroxyl groups [35]. Arylazide functionalized PEG-P(HPMA) block copolymers with a PEG molecular weight of 2, 5 or $12 \mathrm{~kg} \mathrm{~mol}^{-1}$ were prepared to allow investigation of the PEG length on the anti-fouling and bactericidal behavior. The synthesis of aryl-azide functionalized PEG-P(HPMA) with a PEG molecular weight of $5000 \mathrm{~g} \cdot \mathrm{mol}^{-1}\left((\mathrm{PEG}-5 \mathrm{~K})-\mathrm{P}\left(\mathrm{HPMA}-\mathrm{N}_{3}\right)\right)$, which is representative for its analogs (PEG-2K)-P(HPMA-N 3 ) and (PEG-12K)-P(HPMA-N ${ }_{3}$, is discussed below. 
In the first step, the macro-initiator (PEG-5K) 2 -ABCPA was prepared using a previously reported procedure.[28] Its structure was confirmed by ${ }^{1} \mathrm{H}$ NMR (Figure 8.S1). Similarly to our previous finding, the SEC trace of (PEG-5K) $2_{2}$ ABCPA (Figure 8.S2) reveals the presence of a small amount of PEG-5K homopolymer that originates from the macroinitiator synthesis as discussed elsewhere [32]. Other groups showed that the separation of the (PEG-5K) ${ }_{2}-$ ABCPA (10 kg mol$\left.{ }^{-1}\right)$ and PEG-5K homopolymer via common separation techniques, was impractical, expensive and not efficient due to their close molecular weights [33]. For this reason, the macro-initiator (PEG-5K) $2_{2}$ ABCPA was subsequently used without further purification for the preparation of (PEG-5K)-P(HPMA) block copolymer via free radical polymerization of HPMA to allow for the introduction of multiple aryl-azide groups in a next step via modification of the hydroxyl side groups in the P(HPMA) block (Figure 8.2). This absence of purification is not detrimental for the final goal as will be highlighted in the next parts.
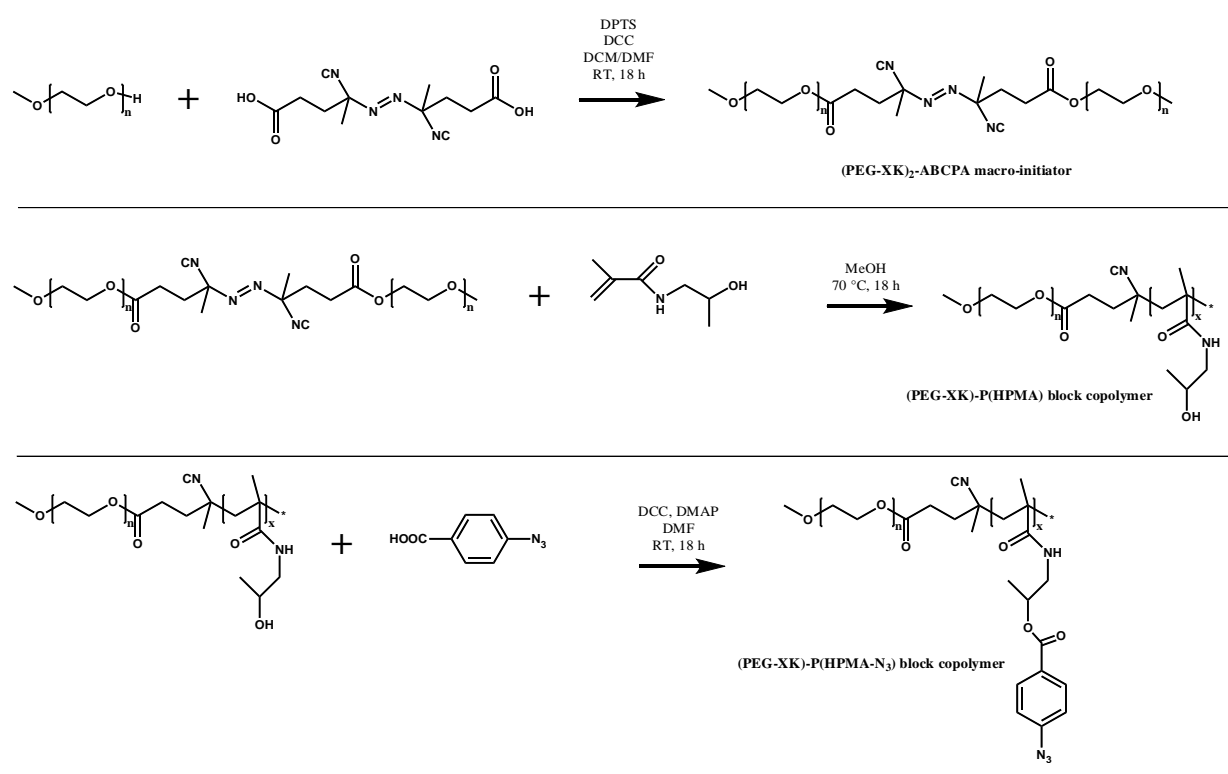

Figure 8.2 - Synthesis scheme for the preparation of (PEG-XK)-P(HPMA-N 3 ). 
An average degree of polymerization (DP) of 40 was calculated by ${ }^{1} \mathrm{H}$ NMR analysis for the P(HPMA) block (copolymer $M_{n} 10.7 \mathrm{~kg} \mathrm{~mol}^{-1}$ ). This result was obtained by comparison between the integral of the peak accounting for the HPMA pendant methine protons and the integral of the peak accounting for PEG protons (Figure 8.S3). SEC analysis of (PEG-5K)-P(HPMA) (Figure 8.S2) shows the presence of PEG-5K that was already present in the starting (PEG-5K) ${ }_{2}$-ABCPA, but that could also be due to chain transfer and primary radical recombination. This chain termination could be limited by using atom transfer radical polymerization (ATRP) [34] and reversible addition-fragmentation chain transfer (RAFT) polymerization [35]. This improvement was not carried out in the frame of this work as PEG-5K is not functionalized with aryl-azide groups in the next step, and therefore is not grafted on the PEEK surface. A $Ð$ of 1.34 was found for (PEG-5K)P(HPMA), close to what was reported elsewhere using a similar procedure [32].

In the final step of the synthetic procedure, aryl-azide groups were introduced via an esterification reaction between 4-azidobenzoic acid and the hydroxyl side groups in the P(HPMA) block. In the ${ }^{1} \mathrm{H}$ NMR spectrum of the resulting (PEG-5K)-P(HPMA-N ${ }_{3}$ ) signals at 7.96 and $7.21 \mathrm{ppm}$ are visible (Figure 8.3A), corresponding to the phenyl protons of the aryl-azide groups. By comparing the ${ }^{1} \mathrm{H}$ NMR integrals of the aromatic protons and methine protons, it was calculated that $50 \%$ of the HPMA units was functionalized with aryl-azide groups. Although no quantitative functionalization was achieved, possibly due to the relatively low amount of 4-azidobenzoic acid in the reaction mixture (equimolar relative to hydroxyl groups) in combination with steric hindrance effects, each (PEG-5K)$\mathrm{P}\left(\mathrm{HPMA}-\mathrm{N}_{3}\right)$ polymer contains approximately 15-20 aryl-azide groups for 
grafting onto PEEK surfaces via UV photoinsertion. The non-quantitative functionalization of (PEG-5K)-P(HPMA-N ${ }_{3}$ ) could be beneficial in view of its grafting to the PEEK surface as a larger distance and hence higher flexibility between the aryl-azide moieties likely promotes multiple attachments to the surface [23].

(A)
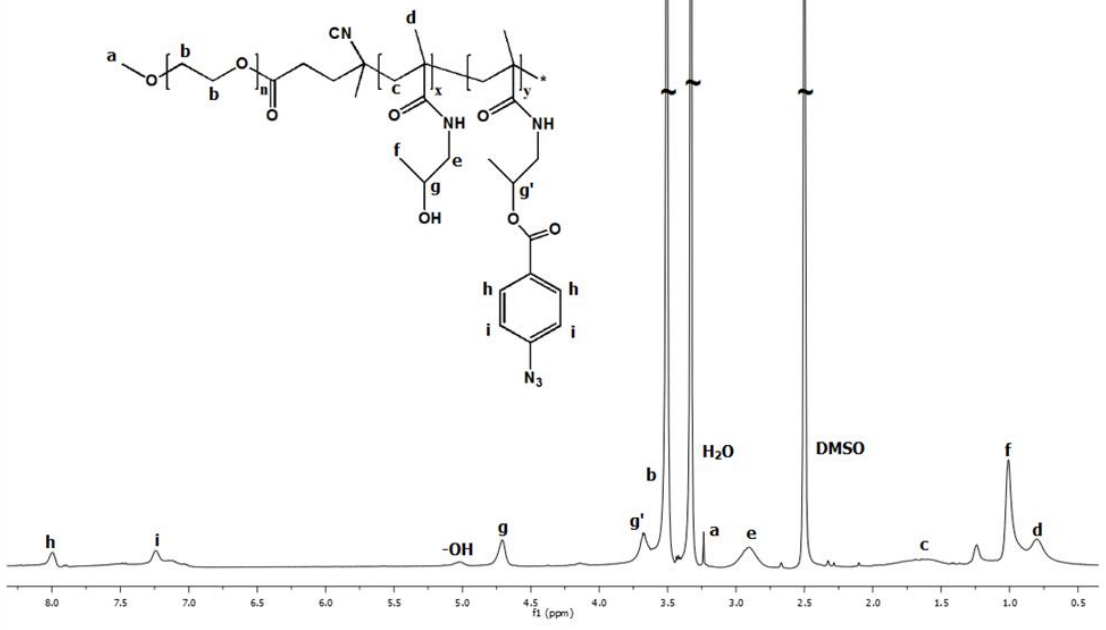

(B)
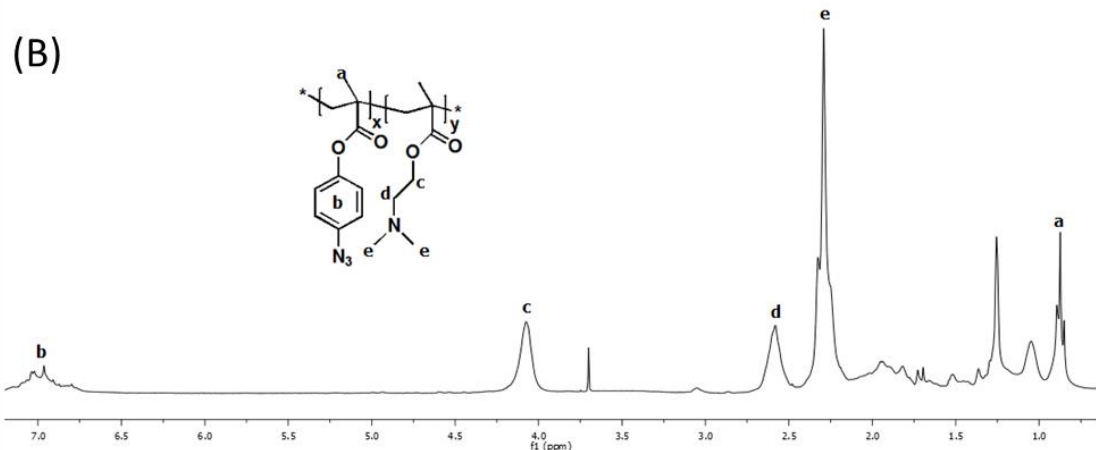

Figure 8.3 - Typical ${ }^{1} \mathrm{H}$ NMR spectra of photoactivable copolymers. Unassigned peaks correspond to backbone protons (Solvent: $\mathrm{CDCl}_{3}$ ).

(A) ${ }^{1}$ H NMR spectrum of (PEG-5K)-P(HPMA-N 3 ) (Solvent: DMSO-d ${ }_{6}$ );

(B) ${ }^{1} \mathrm{H}$ NMR spectrum of P(DMAEMA-co-APM). The signal at $3.70 \mathrm{ppm}$ corresponds to trace amounts of dioxane. 
Following the same three-step synthetic procedure (Figure 8.2), (PEG-2K)$\mathrm{P}\left(\mathrm{HPMA}-\mathrm{N}_{3}\right)$ and (PEG-12K)-P(HPMA-N ${ }_{3}$ ) block copolymers were prepared, having a P(HPMA) DP of 40 and a degree of functionalization of the HPMA units with aryl-azide groups of approximately $75 \%$.

We selected poly(2-(dimethylamino)ethyl methacrylate) P(DMAEMA) [36] as a bactericidal polymer based on our previous study [37] which showed that short $\left(<10 \mathrm{~kg} \mathrm{~mol}^{-1}\right)$ PDMAEMA provides a high antibacterial activity with limited toxic effects on cells. In order to obtain bactericidal polymers which can be grafted onto PEEK surfaces via UV photoinsertion, we synthesized PDMAEMA based polymers with tertiary amines as well as arylazide groups, followed by quaternization of the tertiary amines (Figure 8.4).
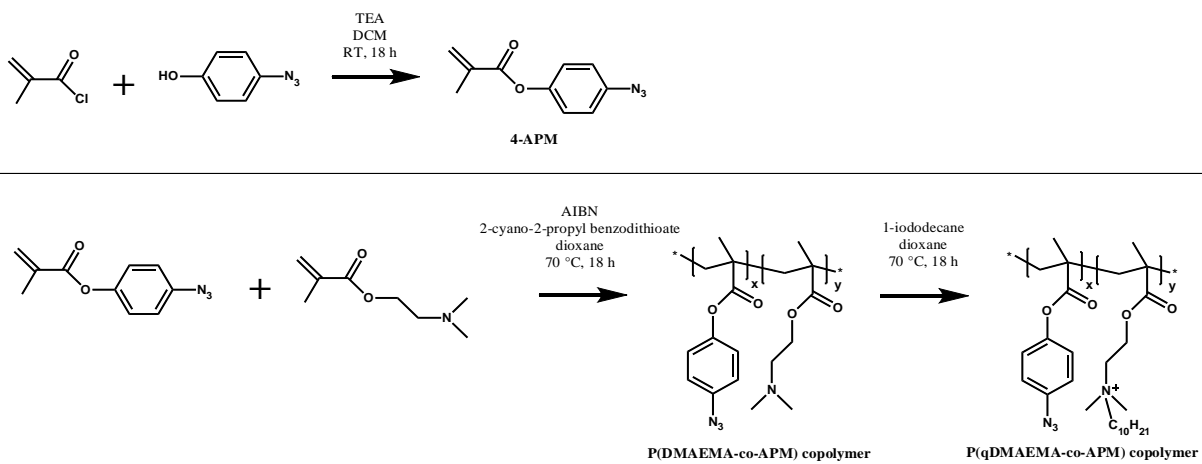

Figure 8.4 - Synthesis scheme for the preparation of P(qDMAEMA-co-APM).

The monomer 4-azidophenyl methacrylate (4-APM) was synthesized by reaction of 4-azidophenol and methacryloyl chloride [27]. After purification, the product was obtained in $60 \%$ yield and its structure was confirmed by ${ }^{1} \mathrm{H}$ NMR spectroscopy (Figure 8.S4). P(DMAEMA-co-APM) copolymer was synthesized by RAFT copolymerization of 4-APM and DMAEMA. Comparison of the ${ }^{1} \mathrm{H}$ NMR spectra of the reaction mixture before and after $18 \mathrm{~h}$ of polymerization indicated full conversion of the monomers (data not 
shown). After purification the polymer was obtained in $32 \%$ yield. By comparing the ${ }^{1} \mathrm{H}$ NMR integrals of the aromatic signals in the 4-APM units and the pendant methylene protons in the DMAEMA units (Figure 8.3B), the DMAEMA/4-APM ratio was calculated to be $2 / 1$, in accordance with the feed ratio. SEC analysis showed a $M_{n}$ of approximately $2.200 \mathrm{~g} \mathrm{~mol}^{-1}$, which is significantly lower than the targeted $M_{n}\left(4.500 \mathrm{~g} \mathrm{~mol}^{-1}\right)$. Since full monomer conversion was achieved, yet the targeted polymer $M_{n}$ was not reached, it is thought that significant oligomerization took place resulting from preliminary primary radical combination and chain transfer. Furthermore, azido groups can undergo cycloaddition to carbon double bonds at higher temperature. These low-molecular weight products are likely removed during purification, which may also explain the relatively low polymer yield (32\%). The limited control over the polymerization results in a relatively high $Ð(2.7)$ as determined by SEC (Figure 8.S5). Li et al. reported good control $(\nexists<1.3)$ over the RAFT homopolymerization of 4-APM and its copolymerization with acrylate monomers at room temperature using the redox initiator system benzoyl peroxide/N,N-dimethylaniline.[27] However, in our experience, RAFT copolymerization of 4-APM with DMAEMA at room temperature resulted in very low conversions of less than $10 \%$. Copolymerization at $70{ }^{\circ} \mathrm{C}$ led to full monomer conversion, but these conditions may also have caused side reactions and a limited control over the copolymerization due to the sensitivity of the azido group to high temperatures. Our current efforts focus on improving the control over the copolymerization of 4-APM and DMAEMA as well as elucidating the arrangement of 4-APM and DMAEMA units along the copolymer chain.

After the synthesis of P(DMAEMA-co-APM), we applied a straightforward quaternization reaction involving 1-iododecane (Figure 8.4). FTIR 
confirmed quaternization of the P(DMAEMA-co-APM) copolymer as signals corresponding to the $\mathrm{C}-\mathrm{H}$ stretching vibrations of 1 -iododecane appeared in the spectrum of P(qDMAEMA-co-APM) (Figure 8.S6). Comparison of the ${ }^{1} \mathrm{H}$ NMR integrals of the aromatic signals in the 4-APM units and the 1-iododecane methylene protons indicated full quaternization of the DMAEMA units (data not shown).

\section{Surface functionalization of PEEK with anti-fouling and bactericidal polymers via photoinsertion}

The PEEK surfaces were analyzed for hydrophilicity by static water contact angle measurements (Figure 8.5). The results demonstrate successful covalent attachment of (PEG-XK)-P(HPMA-N ${ }_{3}$ ) at the surface of the PEEK discs, as UV irradiated, PEG-sprayed surfaces exhibit a lower contact angle, i.e. a higher hydrophilicity, compared to UV irradiated native PEEK. In line with expectations and previous literature, [38,39] the contact angle decreases with increasing PEG chain length. Functionalization of the PEEK surface with $\mathrm{P}(\mathrm{DMAEMA}-\mathrm{co}-\mathrm{APM})$ also results in a lower contact angle $\left(68^{\circ}\right)$. This value is in good agreement with the water contact angle $\left(66^{\circ}\right)$ on a silicon surface functionalized with a copolymer based on DMAEMA and n-butyl methacrylate in a 2:1 ratio [40]. After quaternization of the DMAEMA groups with 1-iododecane the contact angle increases to $93^{\circ}$, despite the presence of hydrophilic quaternary ammonium groups. Previously it was shown that the contact angle depends on the balance between the hydrophilic charged groups and the hydrophobic parts of macromolecules present on a surface [41]. It is hypothesized that in the present study the hydrophilic effect of the charged ammonium groups is outweighed by the hydrophobicity of the long alkyl chains. 


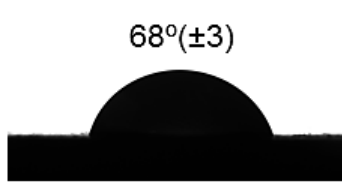

(PEG-2K)-P(HPMA-N $\left.{ }_{3}\right)$

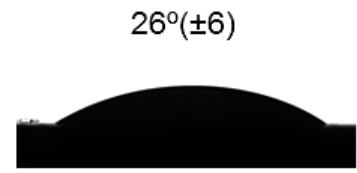

(PEG-5K)-P(HPMA-N 3 ) $18^{\circ}( \pm 4)$

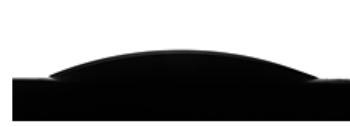

(PEG-12K)-P(HPMA-N $\left.{ }_{3}\right)$

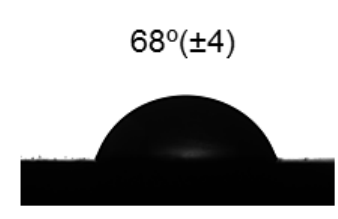

P(DMAEMA-CO-APM)

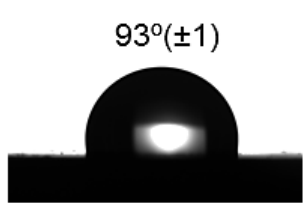

P(qDMAEMA-co-APM)

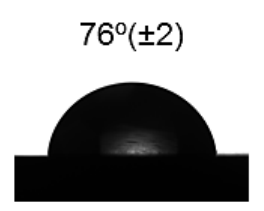

Native PEEK

Figure 8.5 - Contact angles for PEEK surfaces functionalized with various polymers. Contact angles $(n=3)$ are expressed as mean \pm standard deviation.

Upon UV irradiation of (PEG-5K)-P(HPMA-N $\left.{ }_{3}\right)$ and P(qDMAEMA-coAPM) copolymer on PEEK, the FTIR signals corresponding to the $\mathrm{N}_{3}$ stretching vibrations disappear, which confirms the successful photoinsertion of the aryl-azide groups (Figures 8.57 and 8.S8). The emergence of a nitrogen peak in X-ray photoelectron spectra (XPS) of PEEK functionalized with (PEG-5K)-P(HPMA-N 3 ) or P(qDMAEMA-coAPM) (Figure 8.6A) further corroborates the successful grafting of the antifouling and bactericidal polymers. Atomic force microspcopy (AFM) measurements showed that the surface roughness of PEEK increases from $34 \mathrm{~nm}$ to $220 \mathrm{~nm}$ upon functionalization with (PEG-5K)-P(HPMA-N ${ }_{3}$ ) (Figure 8.6B). Overall, the contact angle, FTIR, XPS and AFM experiments clearly demonstrate that PEEK was successfully modified with covalently immobilized anti-fouling (PEG-XK)-P(HPMA-N 3 and bactericidal P(qDMAEMA-co-APM) polymers. 


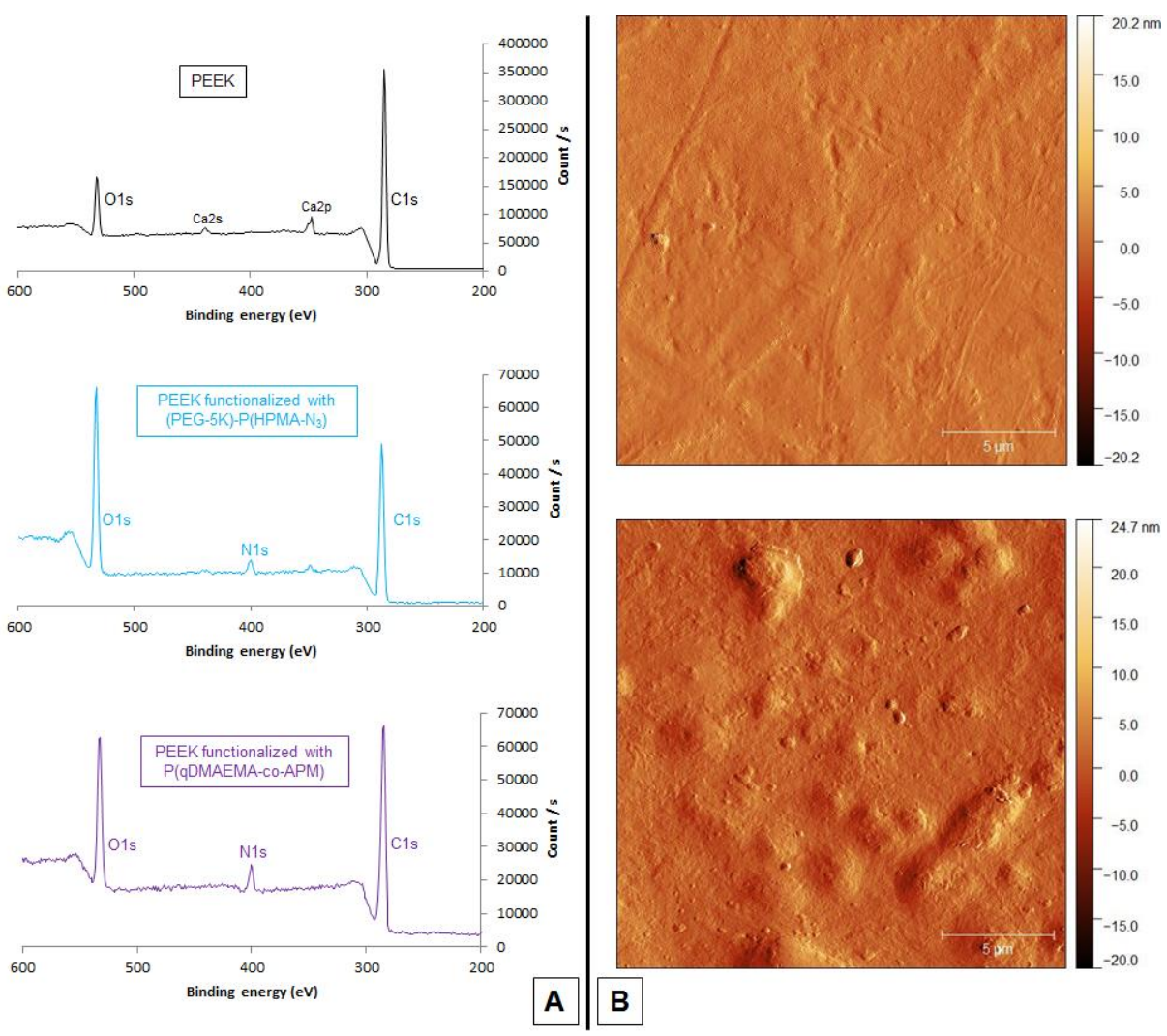

Figure 8.6 - Surface characterization of functionalized PEEK.

A. XPS spectra of PEEK, PEEK functionalized with (PEG-5K)-P(HPMA-N 3 ) and PEEK functionalized with P(qDMAEMA-co-APM).

B. AFM images of unfunctionalized PEEK (top, surface roughness $34 \mathrm{~nm}$ ) and PEEK functionalized with (PEG-5K)-P(HPMA-N 3 ) (bottom, surface roughness 220 $\mathrm{nm})$.

In vitro assessment of anti-fouling and bactericidal properties of functionalized PEEK surfaces

The anti-fouling test against Gram-positive $S$. aureus, presented in Figure 8.7A, revealed that all (PEG-XK)-P(HPMA-N 3 ) coatings could significantly reduce bacterial adhesion after $1 \mathrm{~h}$ of incubation in PBS, with stronger effects observed for longer PEG chain lengths. Against Gram-negative E. coli, the 
effects were less apparent, with the shortest PEG coating lacking significant differences in CFU (colony forming units) attachment compared with native PEEK (Figure 8.7B). For both bacterial strains, increasing PEG chain length negatively affects the ability of bacteria to adhere on the surface of (PEG$\mathrm{XK}-\mathrm{P}\left(\mathrm{HPMA}-\mathrm{N}_{3}\right)$ functionalized PEEK. Thus, the best anti-fouling effect was observed with (PEG-12K)-P(HPMA-N 3 ), which caused a CFU adhesion reduction of $92 \%$ and $60 \%$ for $S$. aureus and E. coli respectively (Figure 8.7A and $8.7 \mathrm{~B})$.

Repeating the same experiment with $S$. aureus in medium containing human serum showed different behavior after $1 \mathrm{~h}$ (Figure 8.7C). Indeed, due to the protein presence in the suspension medium, the initial anti-fouling effect observed in Figures 8.7A and 8.7B was eliminated. Longer incubation time $(24 \mathrm{~h})$ was necessary to observe the anti-fouling effect of (PEG-XK)$\mathrm{P}\left(\mathrm{HPMA}-\mathrm{N}_{3}\right)$ coatings and its previously observed dependence on the chain length, albeit not as pronounced as reported when only PBS was employed as incubating medium. On PEEK surfaces only modified with P(qDMAEMA-co-APM), no diminution of CFU was noted compared to unmodified PEEK over the course of the experiment. For combined coatings, the PEG chain length influences the degree in which the shorter $\mathrm{P}$ (qDMAEMA-co-APM) polymers are exposed to the bacterial suspension and thus their potential bactericidal effect. 
A.

S. aureus

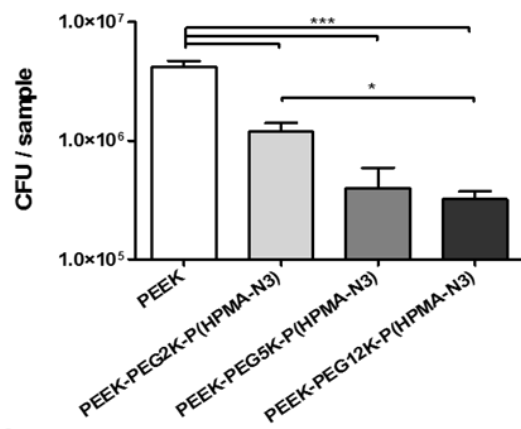

C.

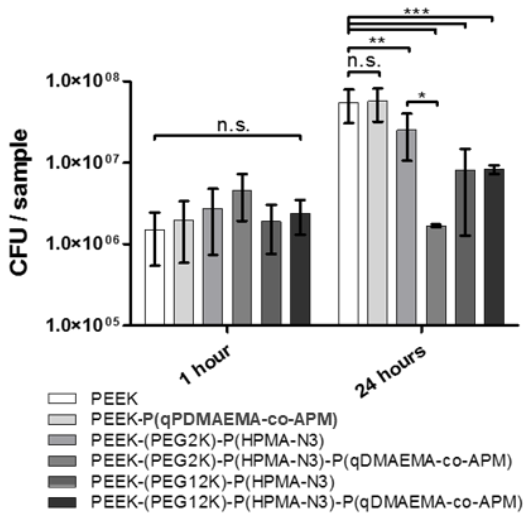

B.

E. coli

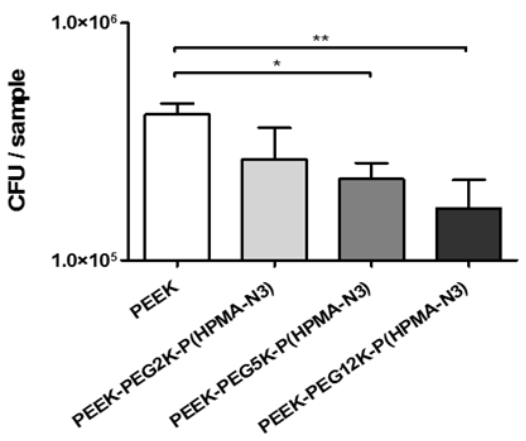

D.

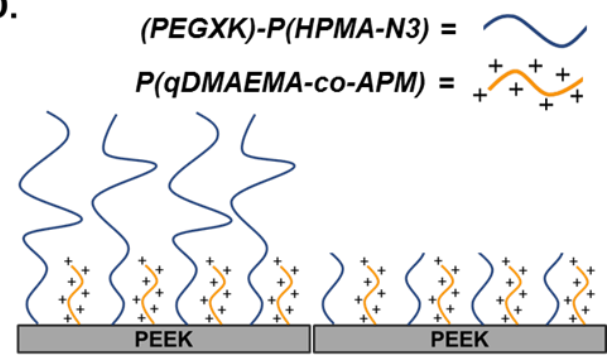

PEG $M_{W}=12.000 \mathrm{~g} / \mathrm{mol} \quad$ PEG $M_{W}=2.000 \mathrm{~g} / \mathrm{mol}$

Figure 8.7 - In vitro anti-fouling and bactericidal testing. All data was subjected to 2 -way ANOVA with Bonferroni post-test $(*=\mathrm{p}<0.05, * *=\mathrm{p}<0.01$ and $* * *=\mathrm{p}<$ 0.001).

A. Assessment of anti-fouling properties of functionalized PEEK surfaces against $S$. aureus.

B. Assessment of anti-fouling properties of the functionalized PEEK surfaces against $E$. Coli.

C. Combined anti-fouling and/or bactericidal effects of (PEG-XK)-P(HPMA-N 3 ), P(qDMAEMA-co-APM) and combined coatings against $S$. aureus over $24 \mathrm{~h}$ in semiphysiological conditions.

D. Schematic representation of (PEG-XK)-P(HPMA-N $)_{3}$ and P(qDMAEMA-coAPM) polymers on PEEK surfaces. 
The bactericidal effect of the P(qDMAEMA-co-APM) chains becomes clearly detectable only when associated with (PEG-2K)-P(HPMA-N $\mathrm{N}_{3}$ ) and after $24 \mathrm{~h}$ of incubation as evidenced by a live/dead staining (Figure 8.S9). A synergistic effect in terms of reduction of $S$. aureus adhesion can thus be seen only when P(qDMAEMA-co-APM) is combined with (PEG-2K)$\mathrm{P}\left(\mathrm{HPMA}-\mathrm{N}_{3}\right)$, and not with the longer (PEG-12K)-P(HPMA-N $\mathrm{N}_{3}$ ) (which exhibited the best anti-fouling property as presented Figure 8.7A and 8.7B). This is evidenced by the similar values of adherent CFU for the (PEG-12K)$\mathrm{P}\left(\mathrm{HPMA}_{3}\right)$ and (PEG-12K)-P(HPMA-N $\left.{ }_{3}\right)+\mathrm{P}(\mathrm{qDMAEMA-co-APM})$ groups. When the PEG chains have an approximately equal size as $\mathrm{P}(\mathrm{qDMAEMA-co-APM}$ ) chains (as is the case for (PEG-2K)-P(HPMA$\left.\mathrm{N}_{3}\right)$ ), close to a 2-log $(99 \%)$ reduction could be observed compared to the PEEK control group. Combination of P(qDMAEMA-co-APM) chains with much longer PEG chains resulted in a loss of bactericidal capacity. This phenomenon can be explained by steric hindrance and masking of the P(qDMAEMA-co-APM) chains, which prevents interaction with the bacterial wall due to the larger size of the PEG chains (Figure 8.7D).

Ishihari et al. reported $91 \%$ reduction in S. mutans adherence on PEEK after grafting poly(2-methacryloyloxyethyl phosphorylcholine) (PMPC) onto the surface of PEEK via aryl-azide UV photoinsertion of PMPC [12]. This value is comparable with the anti-fouling effect we observed for the (PEG-12K)$\mathrm{P}\left(\mathrm{HPMA}-\mathrm{N}_{3}\right)$ functionalized PEEK (92\% reduction for $S$. aureus). The significantly higher reduction in bacterial adhesion (99\%, Figure 8.7C) when PEG is combined with P(qDMAEMA-co-APM) confirms our hypothesis that grafting a combination of anti-fouling polymer and bactericidal polymer is a relevant synergistic approach to prevent bacterial adhesion on PEEK implants. 
To facilitate the clinical translation of any medical device endowed with antiinfectious properties, it is important to assess their stability through sterilization. The chemical stability of the grafted polymers on the PEEK surface was demonstrated by comparing $S$. aureus adhesion on alcohol cleaned discs versus steam sterilized discs (Figure 8.S10). Results between the cleaning and sterilization methods are comparable in terms of CFU reduction of adhesion, with no significant $(\alpha=0.05)$ differences between the two methods. These results confirm that both (PEG-XK)-P(HPMA-N $\left.{ }_{3}\right)$ and P(qDMAEMA-co-APM) coatings are compatible with steam sterilization.

\section{In vitro cytotoxicity of functionalized PEEK surfaces}

The extraction method was used to assess the cytotoxicity of the functionalized PEEK surfaces. Figure 8.8 shows the viability of fibroblast cells treated with undiluted extracts of PEEK samples and various reference materials relative to the untreated control (extraction vehicle), as determined with the CellTiter-Glo luminescence assay. According to the ISO 10993-5 guidelines, a reduction in cell viability of more than $30 \%$ is considered as a cytotoxic effect. Following expectations, $0.25 \%$ zinc dibutyldithiocarbamate polyurethane (ZDBC PU) film as well as $60 \mathrm{mM}$ phenol solution (positive controls) greatly reduce the cell viability. In contrast, fibroblast viability in contact with extracts from the functionalized PEEK surfaces is more than $70 \%$, with values comparable to the toxicity of polyethylene film (negative control) and unfunctionalized PEEK. The cytotoxicity was further evaluated using the lactate dehydrogenase (LDH) activity assay. Figure 8.S11 shows the amount of LDH, a cytosolic enzyme which is released upon cell membrane damage, in the cell culture medium after $24 \mathrm{~h}$. The undiluted extracts of the PEEK samples show a very low toxicity towards fibroblasts after $24 \mathrm{~h}$, 
comparable to the toxicity of the polyethylene extracts (negative control) and the extraction vehicle, corroborating the cytotoxicity results obtained with the CellTiter-Glo luminescence assay. As expected, treatment with diluted extracts of the PEEK samples resulted in similar low cytotoxicity values (data not shown). These in vitro experiments demonstrate that despite clear antifouling and bactericidal effects for the PEEK surfaces (Figure 8.7), the surface functionalization does not induce cytotoxicity for the surrounding medium.

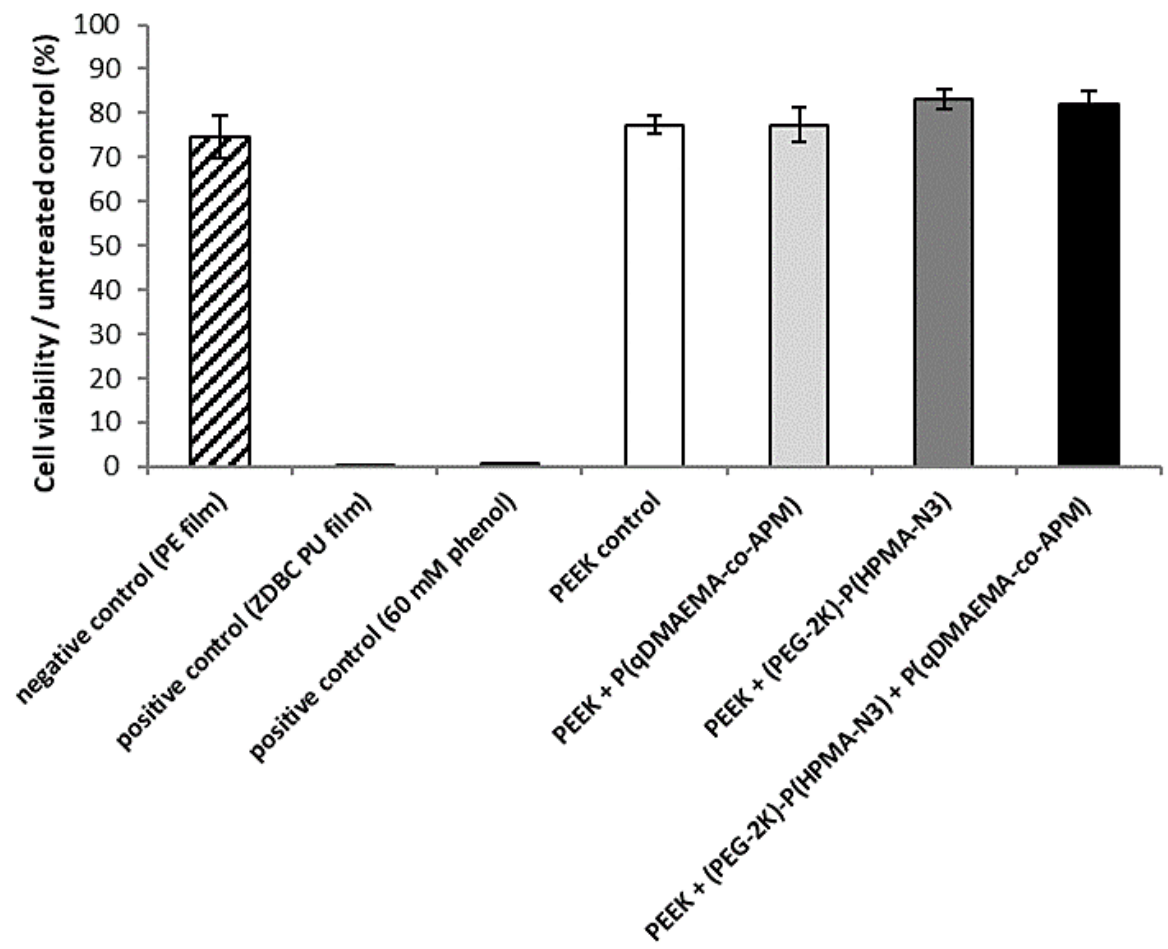

Figure 8.8 - Viability of fibroblasts grown in contact with extracts for $24 \mathrm{~h}$. Data (n $=3$ ) are expressed as mean \pm standard deviation. The viability of cells without any treatment (growth medium only) was taken as $100 \%$. According to the ISO 10993-5 guidelines, a reduction in cell viability of more than $30 \%$ is considered as a cytotoxic effect. 
Perspective of this work will be to validate the antibacterial protection offered by this synergistic combination of anti-fouling and bactericidal polymers under in vivo conditions. In addition, as implants made of PEEK can easily be processed using additive manufacturing based on patient radiographic scans, [4] it would be of interest to validate the developed workflow on preformed patient-specific implants made of PEEK. In this regard, the ability to autoclave the functionalized PEEK samples is highly important for future in vivo assessments and possible clinical application.

\section{Validation of subcutaneous mouse infection model}

As an initial model validation, non-coated PEEK implants (diameter $=5$ $\mathrm{mm}$, height $=2 \mathrm{~mm}$ ) were tested in the subcutaneous murine infection model. While a sigificant deviation of quantified bacteria is observed in the soft tissue, the number of bacteria found on the implants is consistant among 10/12 replicates. With this preliminary study, we can conclude that the used subcutaneous mouse infection model is a valid model to study the antimicrobial and bactericidal effects of functionalized PEEK surfaces.

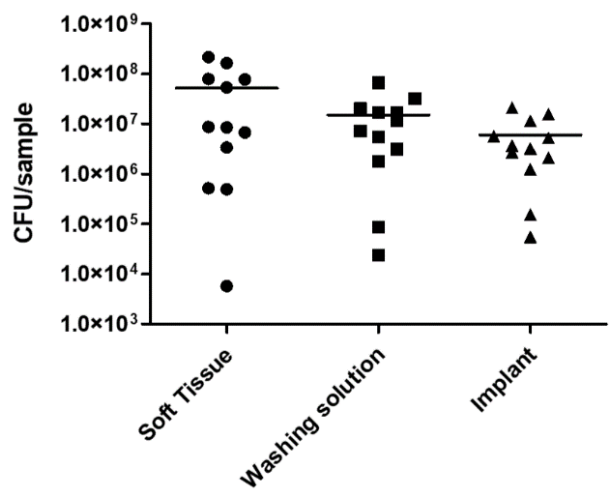

Figure 8.9 - Quantification of $S$. aureus from the murine subcutaneous $S$. aureus infection model with uncoated PEEK implants. 


\section{In vivo assessment of antimicrobial and bactericidal PEEK implants}

Native PEEK (PEEK), PEEK-(PEG-2K)-P(HPMA-N3) (PEEK-PEG), PEEK-P(qDMAEMA-co-APM) (PEEK-PQA) and combined PEEKPEG-PQA coatings were applied on rough (R) and smooth (S) surfaces and tested in the previously described subcutaneous mouse infection model. In Figure 8.10 the quantification of bacteria from the different PEEK implants (A.), washing solutions (B.) and soft tissues (C.) is reported.

The lack of differences of bacteria present on the functionalized PEEK implants (Figure 8.10A) with either rough of smooth surfaces do not correlated well with the in vitro results (Figure 8.7). This is not uncommon and could be due to reduced anti-fouling and antibacterial effect of the modified PEEK surfaces due to high proteins adsorption in the in vivo setup. Chen et al. presented a literature overview of hydrophilic and zwitterionic anti-fouling biomaterials in which a distinction was made between protein absorption and cell adhesion [42]. Even though many materials were able to prevent initial cell adhesion, almost all coatings were subject to significant protein absorption. This protein absorption diminishes the anti-fouling potential of biomaterial coatings over time and facilitates further biofouling of cells and bacteria. In our in vitro set-up we tried to mimic physiological conditions by adding human plasma to a concentration of $2 \% \mathrm{v} / \mathrm{v}$ to the bacterial suspensions that were added to the PEEK implants, with significant reductions of bacterial fouling observed for the combined anti-fouling and bactericidal coating [43]. However, the protein concentration present in the murine subcutaneous environment is most likely higher than what was used in the in vitro experiment. Studies on human subcutaneous protein concentration of edema patients showed that a tot protein concentration of 
$58.4 \mathrm{~g} / \mathrm{L}$ was found in the subcutaneous interstitial fluid [44]. Serum total protein concentration in human plasma, as used in our in vitro experiment, can be averaged at approximately $70 \mathrm{~g} / \mathrm{L}$, resulting in a protein concentration in the bacterial suspensions of just $1.4 \mathrm{~g} / \mathrm{L}$. This discrepancy in protein exposure of the PEEK implants might have contributed to the reduced antifouling and bactericidal properties of the PEEK coatings in the in vivo experiments (Figure 8.10).
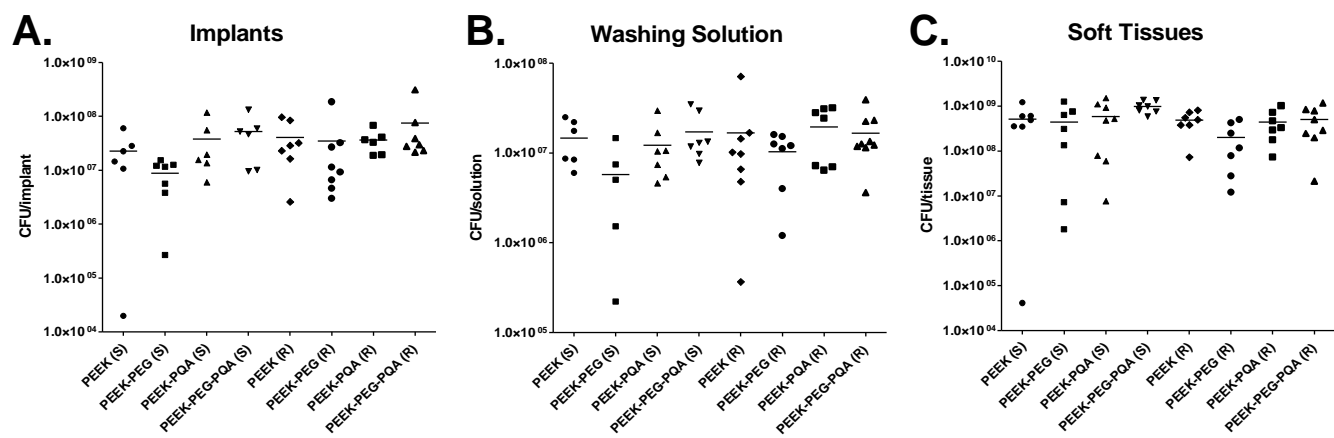

Figure 8.10 - Bacterial quantification from the subcutaneous murine infection model

A. Quantification of $S$. aureus present on the implants.

B. Quantification of $S$. aureus present in washing solutions

C. Quantification of $\boldsymbol{S}$. aureus present in soft tissues surrounding the implant

Definition of implant nomenclature: Native PEEK (PEEK), PEEK-(PEG-2K)P(HPMA-N3) (PEEK-PEG), PEEK-P(qDMAEMA-co-APM) (PEEK-PQA) and combined coatings (PEEK-PEG-PQA).

\section{Conclusion}

We developed a new method to decrease PEEK implant infection by grafting both anti-fouling and bactericidal polymers onto the surface of PEEK via aryl-azide UV photoinsertion. (PEG-XK)-P(HPMA-N 3 ) and P(qDMAEMA-co-APM) were prepared via three-step and two-step procedures, respectively, and their structure was confirmed via ${ }^{1} \mathrm{H}$ NMR. 
Spray coating of polymer solutions onto PEEK and subsequent UV irradiation yielded functionalized PEEK surfaces. The photoinserted polymers on the PEEK surface significantly improved the in vitro anti-fouling and bactericidal properties. A synergistic effect between (PEG-XK)$\mathrm{P}\left(\mathrm{HPMA}-\mathrm{N}_{3}\right)$ and $\mathrm{P}(\mathrm{qDMAEMA}-\mathrm{co}-\mathrm{APM})$ could only be observed when both polymers were of approximately equal chain length. The study also revealed that the presence of proteins in the bacteria-containing medium is an important factor to consider in the evaluation of anti-bacterial surfaces. In vitro cytotoxicity experiments on fibroblasts validated the biocompatibility of the biomaterial as no leachable toxic compounds were released from the modified PEEK samples. These functionalized PEEK surfaces show significant promise in diminishing the susceptibility of native PEEK to bacterial attachment. However, functionalized PEEK implants failed to show similar anti-fouling and bactericidal properties in a subdermal murine infection model that were observed in vitro. The efficacy of the surface coatings was most likely reduced due to biofouling by the high protein concentration in the murine subcutaneous environment. This indicates that the used in vitro experiments are not predictive of the in vivo murine model. Future in vitro experiments may include higher plasma concentrations to better mimic the in vivo environment.

\section{Acknowledgements}

We would like to thank Valérie Flaud (Institut Charles Gerhardt, Montpellier) and Michel Ramonda (Centrale de Technologie en Micro et nanoélectronique, Montpellier) for help with the XPS and AFM measurements, respectively.

\section{Funding Sources}


The authors would like to acknowledge the funding AO CMF-17-02N (PEEK-CAM).

\section{References}

1 Williams, D. F., McNamara, A. \& Turner, R. M. Potential of polyetheretherketone (PEEK) and carbon-fibre-reinforced PEEK in medical applications. Journal of Materials Science Letters 6, 188-190 (1987).

2 Kurtz, S. M. \& Devine, J. N. PEEK biomaterials in trauma, orthopedic, and spinal implants. Biomaterials 28, 4845-4869, doi:10.1016/j.biomaterials.2007.07.013 (2007).

3 Kurtz, S. M. Plastics Design Library. PEEK Biomaterials Handbook.1st edition.Waltham, MA: William Andrew (2012).

4 Alonso-Rodriguez, E. et al. Polyetheretherketone custom-made implants for craniofacial defects: Report of 14 cases and review of the literature. Journal of CranioMaxillofacial Surgery 43, 1232-1238 (2015).

Hanasono, M. M., Goel, N. \& DeMonte, F. Calvarial reconstruction with polyetheretherketone implants. Annals of Plastic Surgery 62, 653-655 (2009).

Rosenthal, G. et al. Polyetheretherketone implants for the repair of large cranial defects: a 3-center experience. Neurosurgery 75, 523-529 (2014).

7 Nemoto, O. et al. Stand-alone anchored cage versus cage with plating for singlelevel anterior cervical discectomy and fusion: a prospective, randomized, controlled study with a 2-year follow-up. European Journal of Orthopaedic Surgery \& Traumatology 25, 127-134 (2015).

8 Cotic, M. et al. A matched-pair comparison of two different locking plates for valgus-producing medial open-wedge high tibial osteotomy: peek-carbon composite plate versus titanium plate. Knee Surgery, Sports Traumatology, Arthroscopy 23, 2032-2040 (2015).

9 Steinberg, E. L. et al. Vol. 17 221-228 (2013).

10 Alexakou, E. et al. PEEK High Performance Polymers: A Review of Properties and Clinical Applications in Prosthodontics and Restorative Dentistry. The European journal of prosthodontics and restorative dentistry 27, 113-121, doi:10.1922/EJPRD_01892Zoidis09 [doi] (2019).

11 Kyomoto, M. et al. Self-initiated surface grafting with poly (2-methacryloyloxyethyl phosphorylcholine) on poly (ether-ether-ketone). Biomaterials 31, 1017-1024 (2010).

12 Ishihara, K. et al. Reliable surface modification of dental plastic substrates to reduce biofouling with a photoreactive phospholipid polymer. Journal of Applied Polymer Science 135, 46512 (2018).

13 Tateishi, T., Kyomoto, M., Kakinoki, S., Yamaoka, T. \& Ishihara, K. Reduced platelets and bacteria adhesion on poly (ether ether ketone) by photoinduced and self-initiated graft polymerization of 2-methacryloyloxyethyl phosphorylcholine. Journal of Biomedical Materials Research Part A 102, 1342-1349 (2014).

14 Amdjadi, P. et al. Ultraviolet-induced surface grafting of octafluoropentyl methacrylate on polyether ether ketone for inducing antibiofilm properties. Journal of Biomaterials Applications 32, 3-11 (2017).

15 Yousaf, A. et al. Vol. 83 70-75 (2014). 
Kakinuma, H. et al. Antibacterial polyetheretherketone implants immobilized with silver ions based on chelate-bonding ability of inositol phosphate: Processing, material characterization, cytotoxicity, and antibacterial properties. Journal of Biomedical Materials Research Part A 103, 57-64 (2015).

Jaekel, D. J. Development and fabrication of silver composite PEEK to prevent microbial attachment and periprosthetic infection. Vol. 74 (2012).

Silver, S. Bacterial silver resistance: molecular biology and uses and misuses of silver compounds. FEMS microbiology reviews 27, 341-353 (2003).

Albers, C. E., Hofstetter, W., Siebenrock, K. A., Landmann, R. \& Klenke, F. M. In vitro cytotoxicity of silver nanoparticles on osteoblasts and osteoclasts at antibacterial concentrations. Nanotoxicology 7, 30-36 (2013).

Mijnendonckx, K., Leys, N., Mahillon, J., Silver, S. \& Van Houdt, R. Antimicrobial silver: uses, toxicity and potential for resistance. Biometals 26, 609-621 (2013).

Rochford, E. T. J. et al. Vol. 113 213-222 (2014).

Klibanov, A. M. Permanently microbicidal materials coatings. Journal of Materials Chemistry 17, 2479-2482 (2007).

23 Schulz, A. et al. Direct Photomodification of Polymer Surfaces: Unleashing the Potential of Aryl-Azide Copolymers. Advanced Functional Materials 28, 1800976 (2018).

24 Dunkin, I., El Ayeb, A., Gallivan, S. \& Lynch, M. 4H-Azepin-4-ones from 4 azidophenols in low-temperature matrices. Journal of the Chemical Society, Perkin Transactions 2, 1419-1428 (1997).

Ulbrich, K. et al. Polymeric drugs based on conjugates of synthetic and natural macromolecules: I. Synthesis and physico-chemical characterisation. Journal of Controlled Release 64, 63-79 (2000).

Wu, H., Yang, Y. \& Cao, Y. C. Synthesis of colloidal uranium- dioxide nanocrystals. Journal of the American Chemical Society 128, 16522-16523 (2006).

Li, G., Zheng, H. \& Bai, R. A facile strategy for the preparation of azide polymers via room temperature RAFT polymerization by redox initiation. Macromolecular rapid communications 30, 442-447 (2009).

Neradovic, D., Van Nostrum, C. F. \& Hennink, W. E. Thermoresponsive polymeric micelles with controlled instability based on hydrolytically sensitive Nisopropylacrylamide copolymers [6]. Macromolecules 34, 7589-7591 (2001).

Khonsari, R. H., Berthier, P., Rouillon, T., Perrin, J.-P. \& Corre, P. Vol. 26 477482 (2014).

30 Järvinen, S. et al. Vol. 47 1072-1076 (2019).

31 Konradi, R., Acikgoz, C. \& Textor, M. Polyoxazolines for nonfouling surface coatings - a direct comparison to the gold standard PEG. Macromolecular rapid communications 33, 1663-1676 (2012).

32 Buwalda, S. et al. Reversibly core-crosslinked PEG-P (HPMA) micelles: Platinum coordination chemistry for competitive-ligand-regulated drug delivery. Journal of colloid and interface science 535, 505-515 (2019).

33 Fliervoet, L. A. L., Najafi, M., Hembury, M. \& Vermonden, T. Heterofunctional Poly (ethylene glycol)(PEG) Macroinitiator Enabling Controlled Synthesis of ABC Triblock Copolymers. Macromolecules 50, 8390-8397 (2017).

34 Matyjaszewski, K. Atom transfer radical polymerization (ATRP): current status and future perspectives. Macromolecules 45, 4015-4039 (2012). 
35 Stenzel, M. H. RAFT polymerization: an avenue to functional polymeric micelles for drug delivery. Chemical Communications, 3486-3503 (2008).

36 Jain, A. et al. Antimicrobial Polymers. Advanced Healthcare Materials 3, 1969-1985, doi:10.1002/adhm.201400418; 22 10.1002/adhm.201400418 (2014).

37 El Habnouni, S. et al. Toward potent antibiofilm degradable medical devices: A generic method for the antibacterial surface modification of polylactide. Acta biomaterialia 9, 7709-7718 (2013).

38 Benhabbour, S. R., Sheardown, H. \& Adronov, A. Protein resistance of PEGfunctionalized dendronized surfaces: Effect of PEG molecular weight and dendron generation. Macromolecules 41, 4817-4823 (2008).

39 Dong, B., Manolache, S., Wong, A. C. L. \& Denes, F. S. Antifouling ability of polyethylene glycol of different molecular weights grafted onto polyester surfaces by cold plasma. Polymer Bulletin 66, 517-528 (2011).

$40 \mathrm{Xu}, \mathrm{C}$. et al. Solution and Surface Composition Gradients via Microfluidic Confinement: Fabrication of a Statistical-Copolymer-Brush Composition Gradient. Advanced Materials 18, 1427-1430 (2006).

41 Berndt, E., Behnke, S. \& Ulbricht, M. Vol. 47 2379-2390 (2011).

42 Chen, S., Li, L., Zhao, C. \& Zheng, J. Surface hydration: Principles and applications toward low-fouling/nonfouling biomaterials. Polymer 51, 5283-5293, doi:https://doi.org/10.1016/i.polymer.2010.08.022 (2010).

43 Buwalda, S. et al. Synergistic anti-fouling and bactericidal poly(ether ether ketone) surfaces via a one-step photomodification. Materials Science and Engineering: C 111, 110811, doi:https://doi.org/10.1016/i.msec.2020.110811 (2020).

44 Haaverstad, R., Romslo, I., Larsen, S. \& Myhre, H. O. Protein concentration of subcutaneous interstitial fluid in the human leg. A comparison between the wick technique and the blister suction technique. Int J Microcirc Clin Exp 16, 111-117, doi:10.1159/000179159 (1996).

\section{Supplementary information and data}

\section{Synthetic procedures}

\section{4-azidophenyl methacrylate}

Briefly, TEA ( $5.8 \mathrm{ml}, 42 \mathrm{mmol})$ was added to a solution of 4-azidophenol $(4.8 \mathrm{~g}, 35 \mathrm{mmol})$ in $\mathrm{DCM}(50 \mathrm{ml})$ at $0{ }^{\circ} \mathrm{C}$ and the mixture was stirred for 5 min. Subsequently a solution of methacryloyl chloride $(3.8 \mathrm{ml}, 39 \mathrm{mmol})$ in $\operatorname{DCM}(5 \mathrm{ml})$ was added dropwise, the reaction mixture was allowed to warm to room temperature and stirred overnight. After the reaction, the mixture was filtered over a fritted glass filter to remove the TEA salt and the organic 
phase was washed with brine, dried with $\mathrm{Na}_{2} \mathrm{SO}_{4}$, filtered and concentrated. The product was purified by column chromatography on silica gel with DCM. 4-APM was obtained as a yellow solid. Yield: $5.1 \mathrm{~g}(60 \%) .{ }^{1} \mathrm{H}$ NMR (300 $\mathrm{MHz}, \mathrm{CDCl}_{3}, \delta$ ): 7.13 (d, 2H, phenyl protons), 7.05 (d, 2H, phenyl protons), $6.35(\mathrm{~s}, 1 \mathrm{H},=\mathrm{CH}), 5.77(\mathrm{~s}, 1 \mathrm{H},=\mathrm{CH}), 2.06\left(\mathrm{~s}, 3 \mathrm{H}, \mathrm{C}\left(\mathrm{CH}_{3}\right)=\mathrm{CH}\right)$.

\section{(PEG-XK) 2 -ABCPA macro-initiators}

(PEG-5K) ${ }_{2}$-ABCPA was synthesized following a previously published procedure [1]. Briefly, ABCPA (0.11 g, $0.40 \mathrm{mmol})$, mPEG5K (4.0 g, 0.80 $\mathrm{mmol}$ ) and DPTS (38 mg, $0.13 \mathrm{mmol}$ ) were dissolved in $22 \mathrm{ml}$ of a mixture of DCM and dry DMF $(10 / 1 v / v)$. After cooling the solution in an ice bath, a solution of DCC $(0.25 \mathrm{~g}, 1.2 \mathrm{mmol})$ in $5 \mathrm{ml}$ DCM was added dropwise under stirring. The mixture was allowed to warm to room temperature and stirred overnight in an argon atmosphere. The reaction mixture was filtered, concentrated and precipitated in cold diethyl ether. (PEG-5K) 2 -ABCPA was obtained by filtration and dried in vacuo to give a white powder. Yield: $3.4 \mathrm{~g}$ (85 \%). ${ }^{1} \mathrm{H}$ NMR $\left(300 \mathrm{MHz}, \mathrm{CDCl}_{3}, \delta\right): 4.25$ (m, 4H, terminal PEG protons), 3.63 (m, 1020H, PEG protons), 3.37 (s, $6 \mathrm{H}, \mathrm{CH}_{3}-\mathrm{O}-\mathrm{CH}_{2}-\mathrm{CH}_{2}$ ), 2.30 - 2.64 (br, 8H, C- $\left.\mathrm{CH}_{2}-\mathrm{CH}_{2}-\mathrm{C}=\mathrm{O}\right), 1.66$ and 1.72 (s, 6H, C- $\left.\mathrm{CH}_{3}\right)$. (PEG-2K)2-ABCPA and (PEG-12K)2-ABCPA were prepared following analogous procedures, starting from $\mathrm{mPEG} 2 \mathrm{~K}$ and $\mathrm{mPEG} 12 \mathrm{~K}$, respectively.

\section{(PEG-XK)-P(HPMA) diblock copolymers}

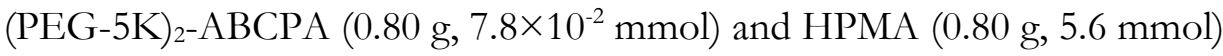
were dissolved in $10 \mathrm{ml}$ of acetonitrile. The solution was stirred at $70{ }^{\circ} \mathrm{C}$ overnight under reflux in an argon atmosphere. After removal of the acetonitrile by rotary evaporation, the product was dissolved in DCM and 
precipitated in cold diethyl ether. (PEG-5K)-P(HPMA) was obtained by filtration and dried overnight in vacuo to give a white powder. Yield: $1.3 \mathrm{~g}$ (83 \%). ${ }^{1} \mathrm{H}$ NMR (300 MHz, $\left.\mathrm{D}_{2} \mathrm{O}, \delta\right): 4.02$ (br, 38H, NH-CH${ }_{2}-\mathrm{CH}\left(\mathrm{CH}_{3}\right)-$ $\mathrm{OH}), 3.80$ (m, 510H, PEG protons), 3.48 (s, $3 \mathrm{H}, \mathrm{CH}_{3}-\mathrm{O}-\mathrm{CH}_{2}-\mathrm{CH}_{2}$ ), 3.1 3.4 (br, 76H, NH-CH${ }_{2} \mathrm{CH}$ ), 1.7 - 2.2 (br, 76H, backbone methylene protons), 1.29 (br, $\left.114 \mathrm{H}, \mathrm{NH}-\mathrm{CH}_{2}-\mathrm{CH}\left(\mathrm{CH}_{3}\right)-\mathrm{OH}\right), 1.0-1.2$ (br, $114 \mathrm{H}$, backbone methyl protons). SEC: $M_{n} 8800 \mathrm{~g} \mathrm{~mol}^{-1}, Ð$ 1.34. (PEG-2K)$\mathrm{P}(\mathrm{HPMA})$ and (PEG-12K)-P(HPMA) were prepared following analogous procedures, starting from (PEG-2K) ${ }_{2}-\mathrm{ABCPA}$ and $(\mathrm{PEG}-12 \mathrm{~K})_{2}-\mathrm{ABCPA}$, respectively.

\section{(PEG-XK)-P(HPMA-N 3 ) diblock copolymers}

For the synthesis of (PEG-5K)-P(HPMA-N 3 ), (PEG-5K)-P(HPMA) (0.75 g, $8.1 \times 10^{-2} \mathrm{mmol}$ ), 4-azidobenzoic acid (0.43 g, $\left.2.6 \mathrm{mmol}\right)$ and DMAP $(38 \mathrm{mg}$, $0.31 \mathrm{mmol}$ ) were dissolved in $10 \mathrm{ml}$ dry DMF. After cooling the solution in an ice bath, a solution of DCC $(0.54 \mathrm{~g}, 2.6 \mathrm{mmol})$ in $5 \mathrm{ml}$ DMF was added dropwise under stirring. The mixture was allowed to warm to room temperature and stirred overnight in an argon atmosphere. The reaction mixture was filtered, concentrated and precipitated in cold diethyl ether. (PEG-5K)-P(HPMA-N ${ }_{3}$ ) was obtained by filtration and dried in vacuo to give a light brown powder. Yield: $0.73 \mathrm{~g}(62 \%)$.

${ }^{1} \mathrm{H}$ NMR (300 MHz, DMSO-d 6 , 8$): 7.96$ (br, 38H, phenyl protons), 7.21 (br, $38 \mathrm{H}$, phenyl protons), 5.00 (br, $\left.19 \mathrm{H}, \mathrm{NH}-\mathrm{CH}_{2}-\mathrm{CH}\left(\mathrm{CH}_{3}\right)-\mathrm{O}-\mathrm{C}=\mathrm{O}\right), 4.72$ (br, $\left.19 \mathrm{H}, \mathrm{CH}\left(\mathrm{CH}_{3}\right)-\mathrm{OH}\right), 3.51$ (m, 510H, PEG protons), 3.24 (s, $3 \mathrm{H}$, $\mathrm{CH}_{3}-\mathrm{O}-\mathrm{CH}_{2}-\mathrm{CH}_{2}$ ), 2.90 (br, 76H, NH-CH${ }_{2}-\mathrm{CH}$ ), 1.4 - 2.0 (br, 76H, backbone methylene protons), 0.99 (br, $\left.114 \mathrm{H}, \mathrm{NH}-\mathrm{CH}_{2}-\mathrm{CH}\left(\mathrm{CH}_{3}\right)-\mathrm{O}\right), 0.7$ - 
0.9 (br, 114H, backbone methyl protons). (PEG-2K)-P(HPMA-N ${ }_{3}$ ) and (PEG-12K)-P(HPMA-N $\mathrm{N}_{3}$ ) were prepared following analogous procedures, starting from (PEG-2K)-P(HPMA) and (PEG-12K)-P(HPMA), respectively.

\section{Characterization of functionalized PEEK surfaces}

FTIR spectra were recorded using a Perkin-Elmer Spectrum 100 FTIR spectrometer. Static water contact angles were obtained via the sessile drop method. A PEEK disc was placed in a system consisting of a sample holder, a light source and a Dataphysics OCAH200 CDD camera. Subsequently one drop of distilled water (approximately $50 \mu \mathrm{l}$ ) was placed on the PEEK disc using a pipette. The drop profile was captured with the camera and analyzed with ImageJ 1.51 software employing the DropSnake plug-in. Three sample points were collected for each PEEK sample. XPS analyses were performed on an ESCALAB 250 photoelectron spectrometer (Thermo Electron). The excitation source was the $\mathrm{Al} \mathrm{K \alpha} \mathrm{X}$-ray at $1486.6 \mathrm{eV}$. The spectra were collected from a surface with a diameter of $500 \mu \mathrm{m}$ and calibrated to the binding energy of the C-C component of the $C_{1 \mathrm{~s}}$ peak to $284.8 \mathrm{eV}$. Atomic Force Microscopy (AFM) studies were performed using a Nanoman atomic force microscope (Bruker Metrology, Santa Barbara, USA) equipped with a closed loop and run by Nanoscope 5 software (Bruker Instruments). Using an NCL cantilever (Nanosensors, Neuchatel, Switzerland) images were recorded in tapping mode under ambient conditions. Images were processed using Gwyddion 2.50 software. 


\section{Evaluation of cytotoxicity via extraction method (ISO 10993-5) Extract preparation}

PEEK samples $(\mathrm{n}=3)$ were autoclaved and Dulbecco's modified Eagle's medium (Sigma, $4.5 \mathrm{~g} \mathrm{l}^{-1} \mathrm{D}$-glucose) supplemented with $5 \%$ fetal bovine serum (Gibco), $2 \mathrm{mM} \mathrm{L-glutamine} \mathrm{(Gibco),} 100$ units $\mathrm{ml}^{-1}$ penicillin (Sigma) and $1 \%$ streptomycin (Sigma), named extraction vehicle, was added to achieve an extraction ratio of $3 \mathrm{~cm}^{2} \mathrm{ml}^{-1}$ (ISO 10993-12). Extraction was performed for $72 \mathrm{~h}$ at $37^{\circ} \mathrm{C}$ under stirring. The same protocol was used to prepare extracts from standard reference materials (ISO 10993-5, $\mathrm{n}=3$ ). In this study, high density polyethylene film and $0.25 \%$ zinc dibutyldithiocarbamate (ZDBC) polyurethane film (both from Hatano Research Institute, Japan) were used as negative and positive control, respectively.

\section{Test on L929 cells}

L929 fibroblasts, tested to be free of mycoplasma, were seeded at $1 \cdot 10^{4}$ cells per well (96-well plate) and allowed to adhere overnight. Subsequently extracts were added to the L929 cell monolayer. The following concentrations of the test item extracts were tested: $25 \%, 50 \%, 75 \%$ and $100 \%(v / v)$. For the negative control only the $100 \%$ extract was tested. Dilutions were prepared using the extraction vehicle. The cell viability was assessed after $24 \mathrm{~h}$ incubation at $37^{\circ} \mathrm{C}$ under humidified atmosphere with 5 $\% \mathrm{CO}_{2}$ using the CellTiter-Glo luminescence assay (Promega) and the lactate dehydrogenase activity assay (LDH assay, Pierce). Briefly, for the CellTiterGlo luminescence assay, CellTiter-Glo reagent was added in each well with a volume equal to the volume initially present in each cell culture well. After 10 min incubation at room temperature, luminescence was recorded on a 
BMG Labtech CLARIOstar ${ }^{\circledR}$ microplate-reader. The luminescent signal is proportional to the amount of ATP present in cell medium after cell lysis and hence to the number of cells in the well. For the LDH assay, equal amounts of cell supernatant and LDH reaction mixture were mixed and incubated for $30 \mathrm{~min}$ at room temperature. Subsequently, the absorbance was read at $490 \mathrm{~nm}$ using a BMG Labtech CLARIOstar ${ }^{\circledR}$ microplate-reader.

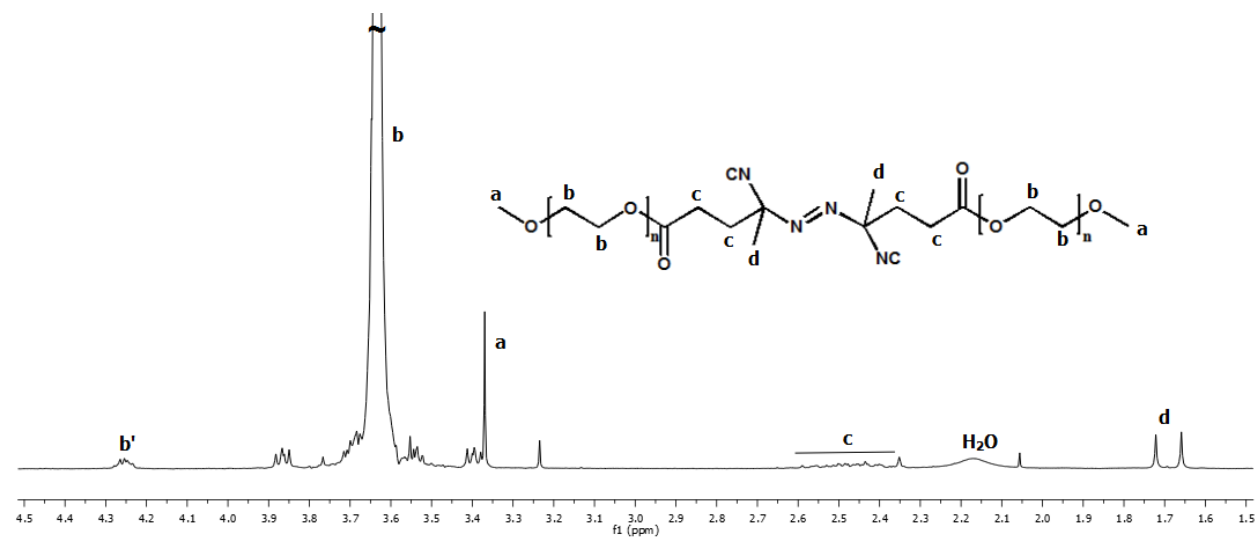

Figure 8.S1. ${ }^{1} \mathrm{H}$ NMR spectrum of (PEG-5K) ${ }_{2}$-ABCPA macro-initiator. Peak b' corresponds to terminal PEG protons. Solvent: $\mathrm{CDCl}_{3}$.

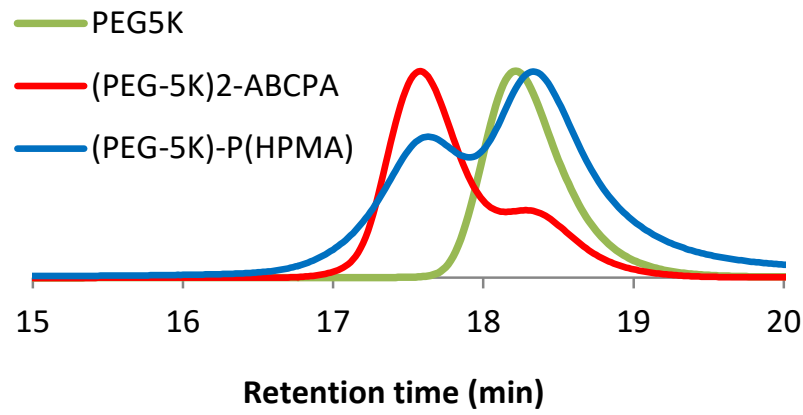

Figure 8.S2. Normalized SEC traces of PEG-5K, (PEG-5K) 2 -ABCPA and (PEG5K)-P(HPMA). 


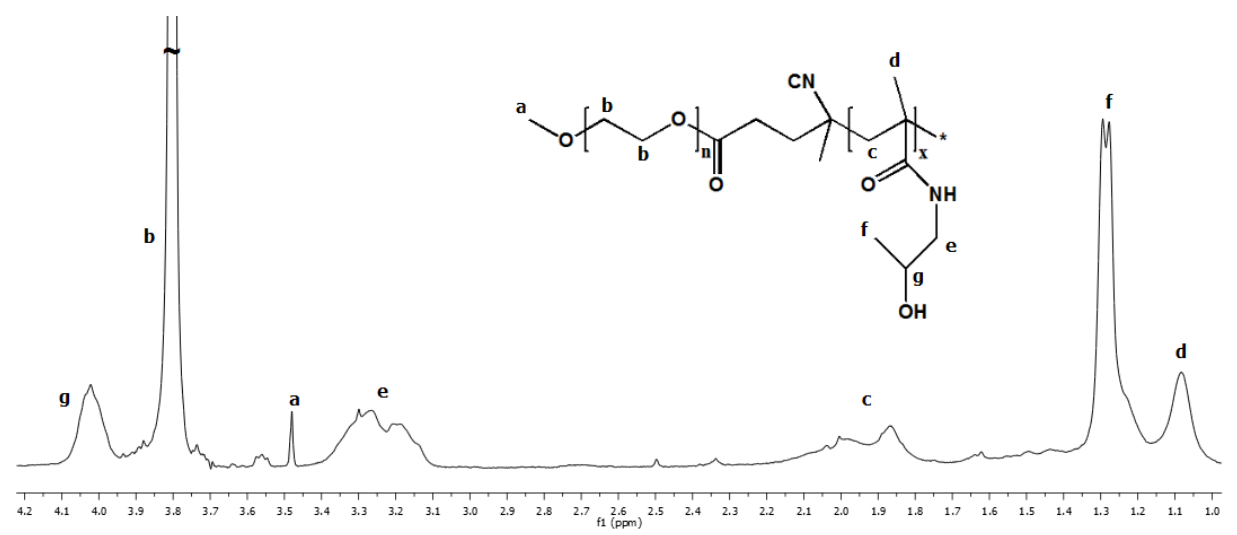

Figure 8.S3. ${ }^{1} \mathrm{H}$ NMR spectrum of (PEG-5K)-P(HPMA) block copolymer. Unassigned peaks correspond to $\mathrm{P}(\mathrm{HPMA})$ backbone protons. Solvent: $\mathrm{D}_{2} \mathrm{O}$.
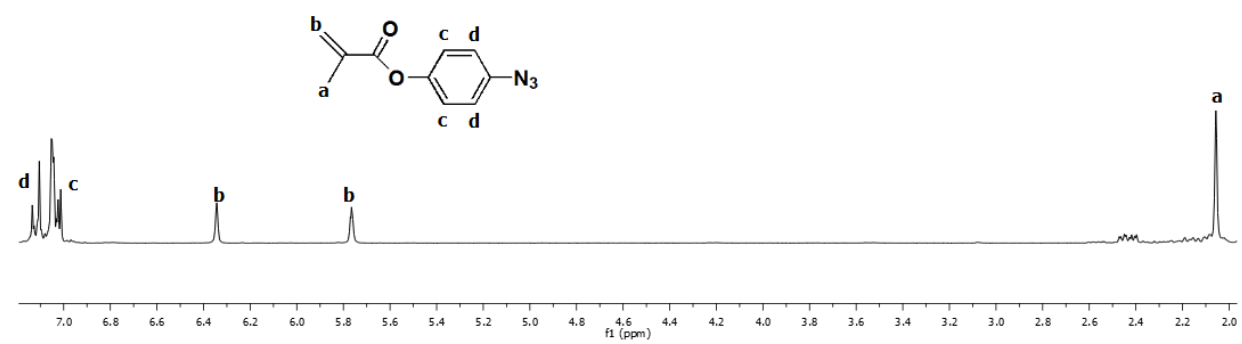

Figure 8.S4. ${ }^{1} \mathrm{H}$ NMR spectrum of 4-azidophenyl methacrylate (4-APM). Solvent: $\mathrm{CDCl}_{3}$.

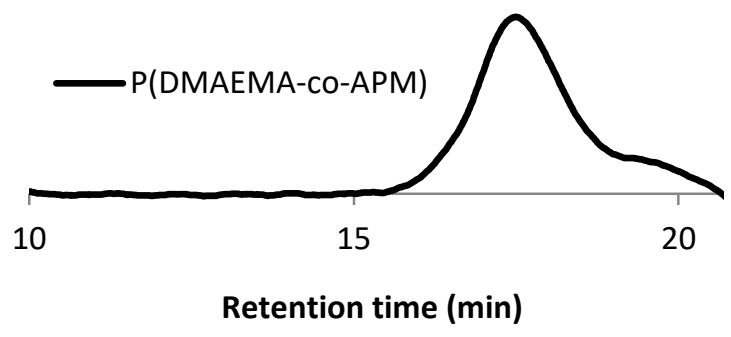

Figure 8.S5. SEC trace of P(DMAEMA-co-APM). 


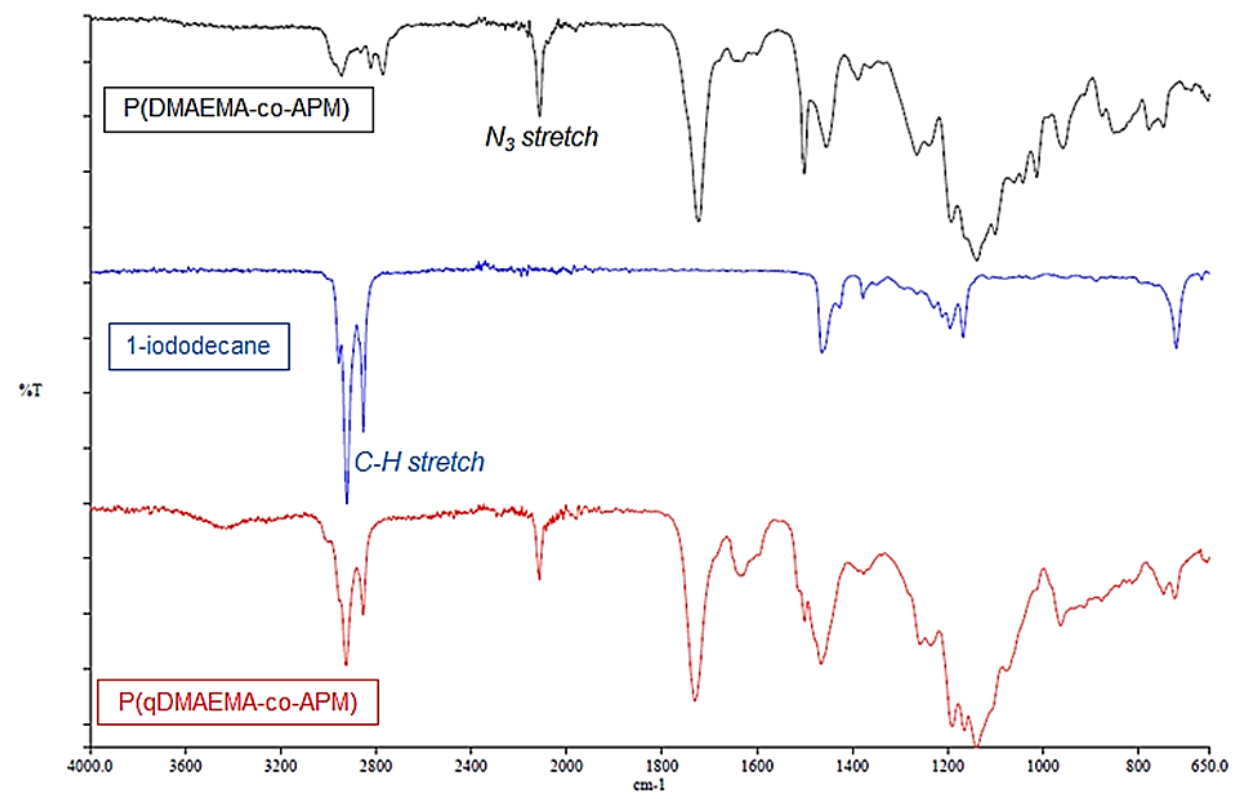

Figure 8.S6. FTIR spectra of P(DMAEMA-co-APM), 1-iododecane and P(qDMAEMA-co-APM).

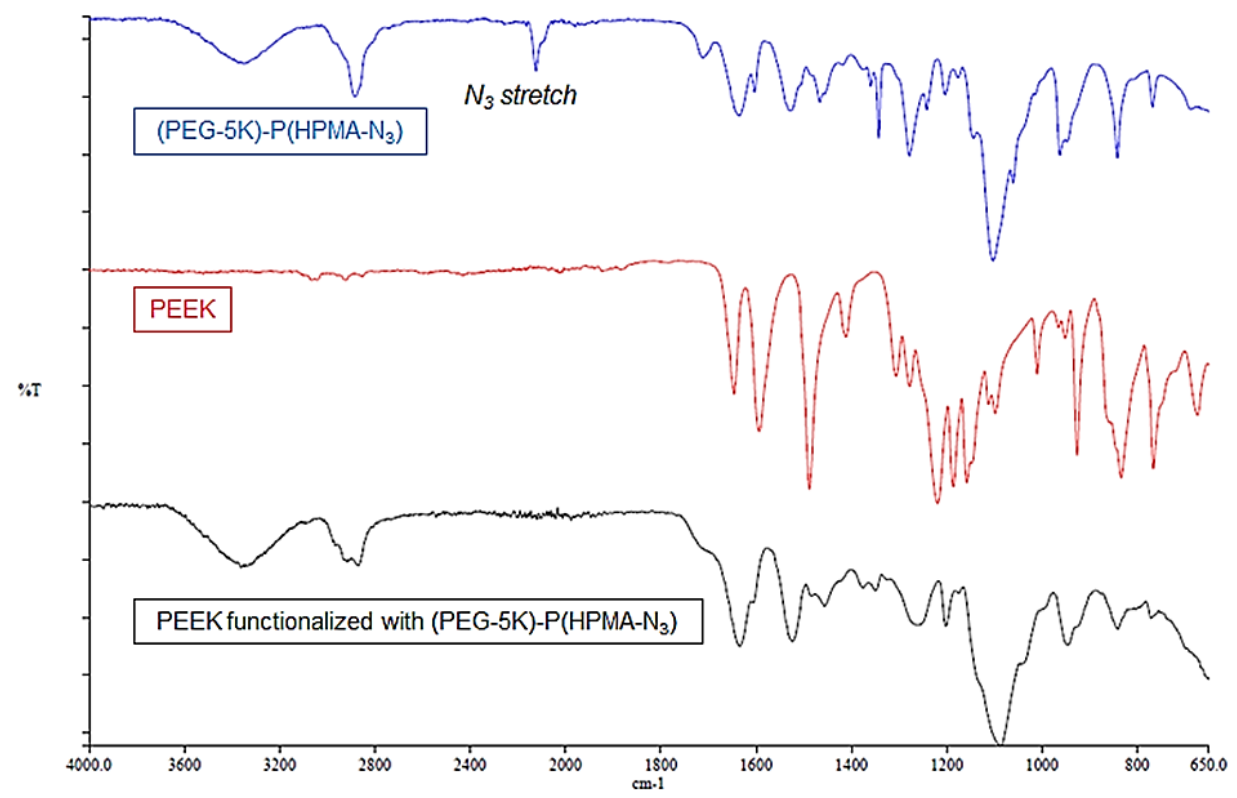

Figure 8.S7. FTIR spectra of (PEG-5K)-P(HPMA-N 3$)$, PEEK, and PEEK functionalized with (PEG-5K)-P(HPMA-N 3 ). 


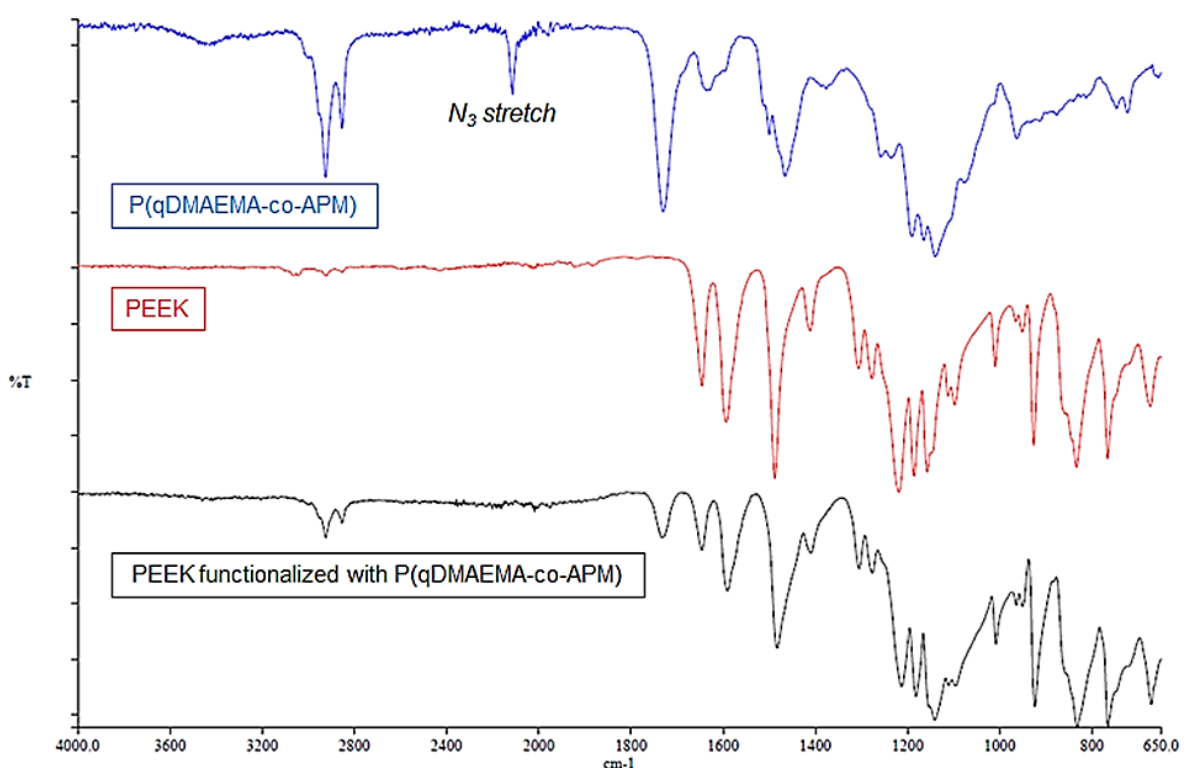

Figure 8.S8. FTIR spectra of P(qDMAEMA-co-APM), PEEK and PEEK functionalized with P(qDMAEMA-co-APM).
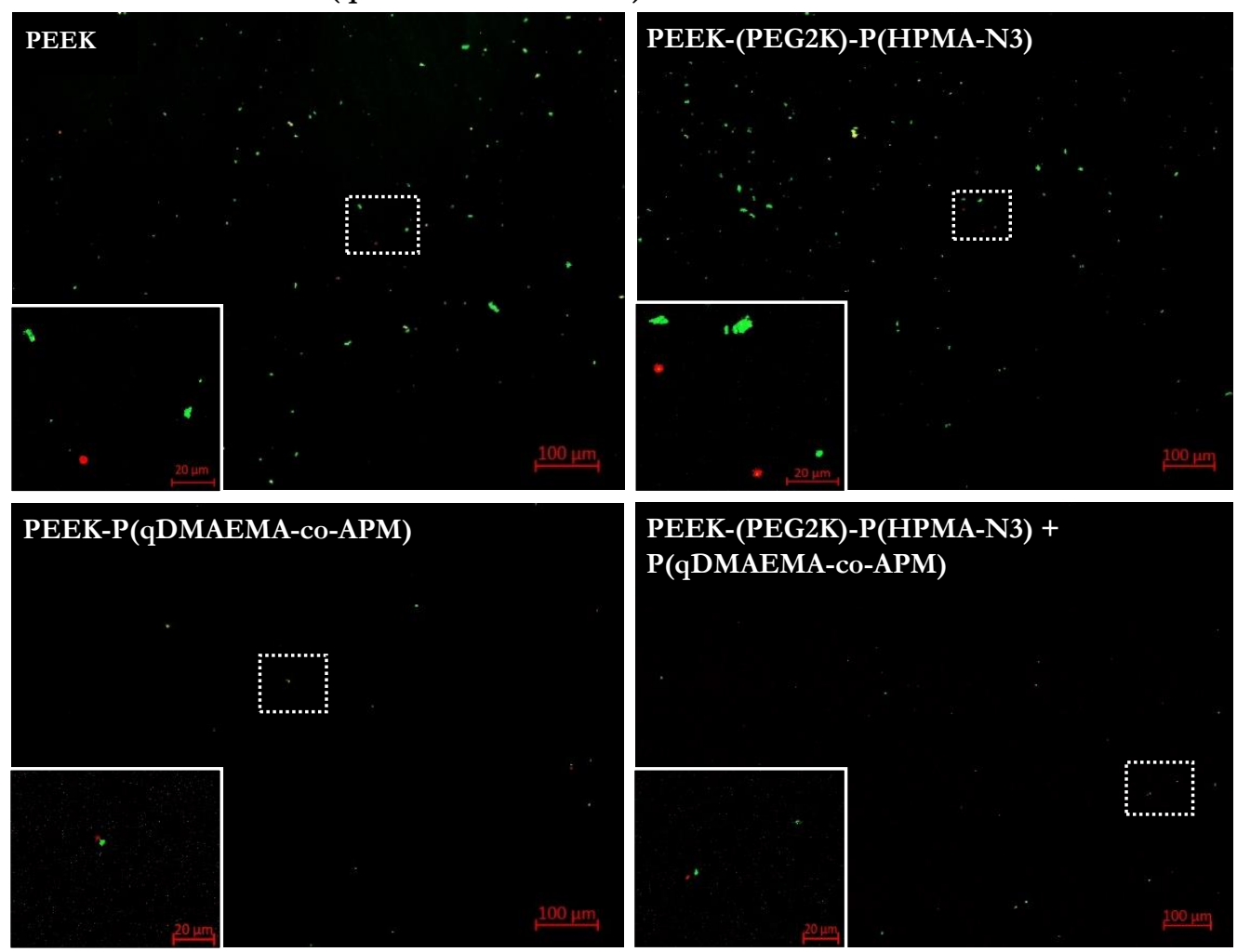

Figure 8.S9. Live/Dead staining of bacteria cultured for 24 hours on PEEK surfaces. 


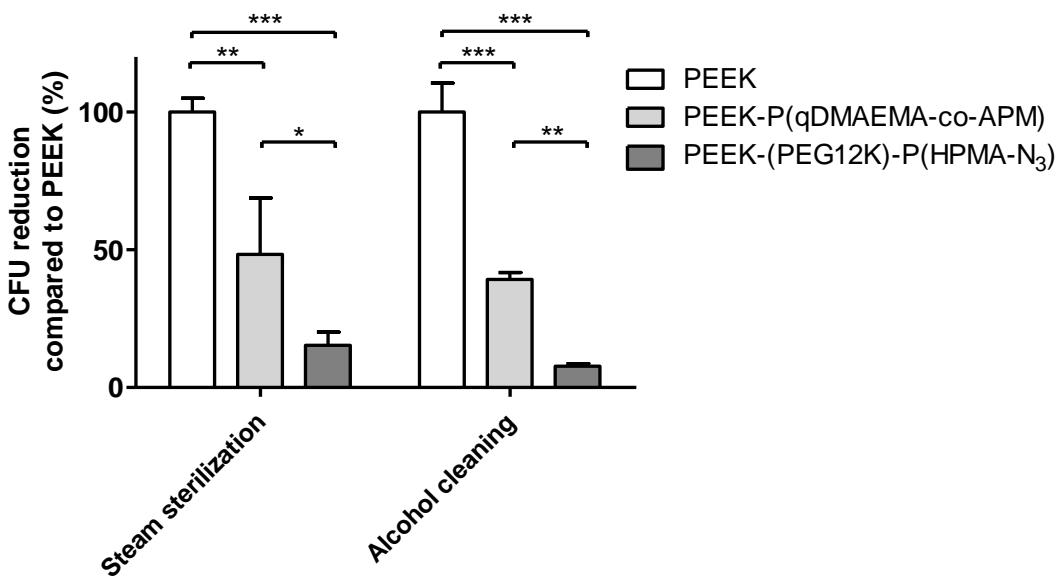

Figure 8.S10. Direct assessment of cleaning and sterilization compatibility of the grafted polymers on the PEEK surface. Results within each method were normalized to the corresponding PEEK group. The data was subjected to 2-way ANOVA with Bonferroni post-test $(*=\mathrm{p}<0.05, * *=\mathrm{p}<0.01$ and $* * *=\mathrm{p}<0.001)$.

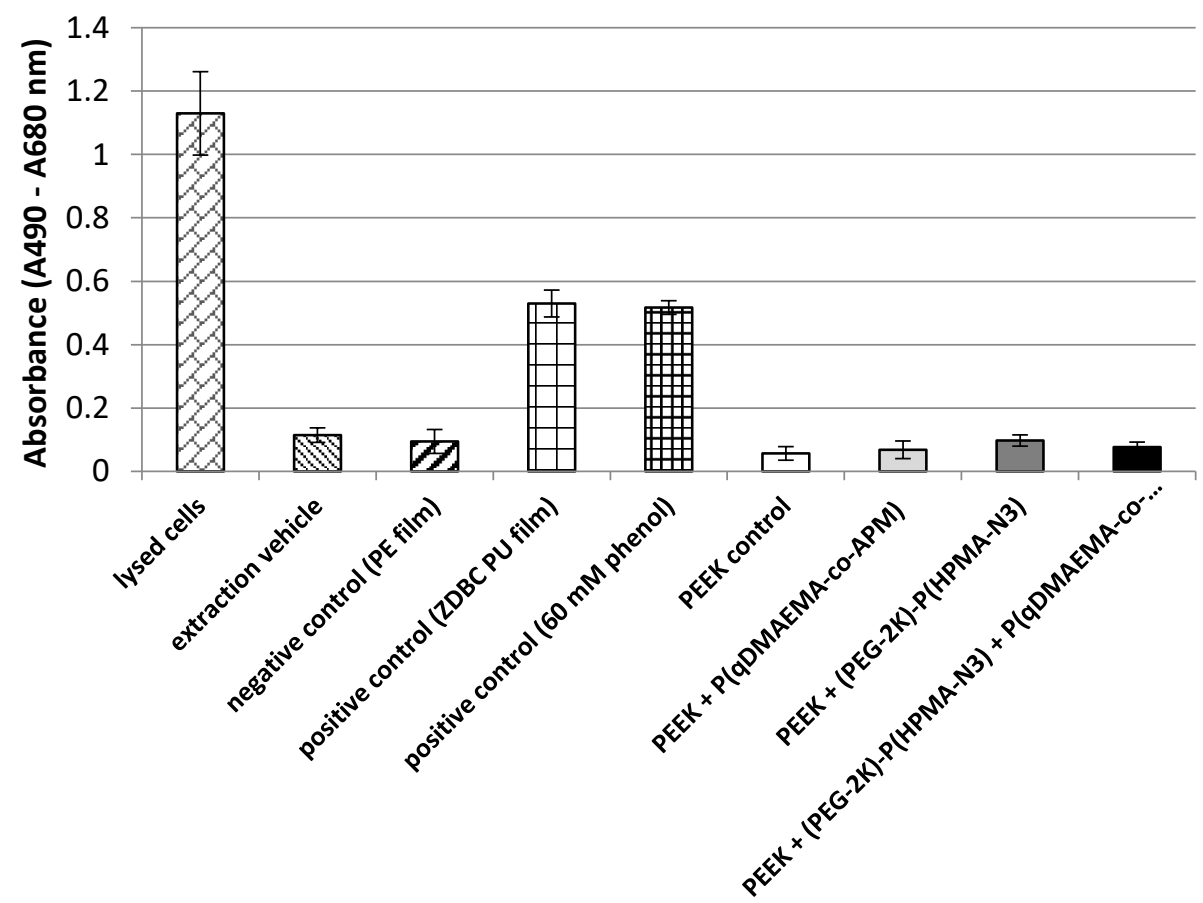

Figure 8.S11. LDH cytotoxicity assay of fibroblasts grown in contact with extracts for $24 \mathrm{~h}$. Data $(n \geq 3)$ are expressed as mean \pm standard deviation. 
Chapter 9

General discussion and future perspectives 


\section{General discussion}

While bacterial infectious diseases can cause systemic host reactions, the infection pathology is often of a local nature. The efficacy of systemic antibiotic treatments is thus heavily reliant on the vascularity of the local infection site. In the case of osteomyelitis $(\mathrm{OM})$, the vascularity of the affected necrotic bone or the debridement site is severely impaired, limiting the efficacy of systemic antibiotics. Similarly, orthopedic implants such as fracture fixation devices or defect fillers are especially susceptible to bacterial colonization due to the lack of vascularity and reduced immunological responses at the implant interface. Such avascular environments are likely to benefit from local administration of antibiotics, i.e. direct placement if antibiotic within the affected tissue. Ever since the 1970's, antibiotic-loaded biomaterials for local implantation have contributed to improved OM treatment outcomes, but treatment failure occurs relatively frequently with an infection recurrence rate of $20-30 \%$ within a year [1]. Antibiotic release from biomaterials is most often diffusion-driven or in some situations established through degradation of the biomaterial drug carrier [2]. In both scenarios, proximity of the antibiotic loaded biomaterial to the infected tissue would be beneficial to maintain high local concentrations of the released antibiotic, with no adverse systemic side effect due to low antibiotic serum concentrations.

To achieve high antibiotic concentrations at the site of OM, bone-targeting microsphere delivery systems have been fabricated in this thesis and extensively tested in vitro. Tailoring antibiotic release from such delivery systems can be achieved by selecting or altering the material properties and microsphere characteristics, including molecular weight, 
hydrophilicity/hydrophobicity, material density/porosity and microsphere diameter. In this work we investigated different fabrication strategies with poly( $\epsilon$-caprolactone) (PCL) and poly(L-lactic acid), eventually settling on an $\mathrm{O} / \mathrm{W}$ emulsion of PCL solutions prepared by probe sonication. Future work could include the alteration of antibiotic diffusion and microsphere biodegradation through utilization of polymer blends with higher hydrophilicity than those used in this thesis or by designing porous microspheres. These additional parameters could be investigated in the pursuit of optimized microspheres for sustained antibiotic release. A major difficulty lies in the lack of scientific consensus on the optimal dosage and duration of local antibiotic therapies [3]. Having this knowledge would be of tremendous benefit for the optimization of antibiotic delivery systems and their efficacy in vivo.

As bone targeting moieties, bisphosphonates (BP) molecules are the most frequently employed in current bone binding (pro-)drugs and commercial fluorescent bone-markers [4]. When using BP's as bone targeting groups for drug delivery, potential therapeutic side effects on the bone remodeling capacity must be carefully considered [5]. That is why short oligopeptides of aspartic acid (ASP), which have no known adverse therapeutic side effects and near equal affinity to hydroxyapatite (HAP), have been implemented in Chapter 4 as alternative bone targeting moieties. [6]. The increased efficacy of delivering antibiotics with PCL/cPVA-ASP compared to nonfunctionalized PCL/cPVA, as seen in Chapter 4, provided the proof of concept for our antibiotic delivery systems and should be further tested in pre-clinical infection models. 
In Appendix A, Gentamicin-AOT (GM-AOT) was loaded in PCLpoly(ethylene glycol) (PCL-PEG) micelle nanoparticles. Antibiotic release from PCL-PEG nanoparticles was rapid with a near complete antibiotic release observed in 48 hours. This fast release kinetic of GM-AOT could make for a good prophylactic approach in orthopedic surgery, however for implementation as a treatment for $\mathrm{OM}$ the release of antibiotic was arguably too fast. Hence, the $\mathrm{O} / \mathrm{W}$ emulsion fabrication of antibiotic loaded PCL microspheres was favored in further assessments of HAP affinity.

Additional applications of the bone targeting drug delivery systems as implant coatings could be envisioned. Many long-term prosthesis that aim for osteointegration are coated with mineralized surfaces [7] which could be coated with the developed drug delivery systems. Previous advances in antimicrobial coating of prosthesis include surface adsorbed antibiotics with complete release reached in 24 hours [8]. Stacked coatings in which antibiotics and PLGA is spray-dried on HAP coatings have also been developed showing antibiotic release over 7 days [9]. However, the PLGA coating does mask the biological effects of the underlying HAP coating until the PLGA is sufficiently degraded. Antimicrobial coatings implementing antibiotic loaded microspheres would be able to be dosed to a desired surface coverage and antibiotic presence to maintain antimicrobial properties for several days, as was evidenced by release studies in Chapter 3 and Chapter 4 .

Even though more efficient antibiotic therapy is beneficial in slowing the development of antibiotic resistance of bacteria, alternative antimicrobials for the treatment of antibiotic resistant infections is of increasing urgency. In Chapter 5 bacteriophages (viruses of microbes) are introduced as alternative antimicrobials with potential to treat antibiotic resistant 
infections. As bacteriophages benefit from hydrated environments, hydrogels have been investigated as a bacteriophage delivery platform. Thermo-responsive HA-pNIPAM hydrogels can be injected at the infected bone site and transition into a gelled state after the hydrogel has flowed into the infected site, facilitating close-contact delivery of its antimicrobial payload. In contrast to many antibiotics, bacteriophages are characterized by amplification cycle upon bacterial infection. After a phage successfully infects a bacterial host cell, the bacteriophage can be extensively replicated before the bacteria is lysed, meanwhile phage can also be inactivated due to in vivo environmental cues which brings balance to the phage titers present at an infection site. Therefore, bacteriophages can be seen as a self-dosing antimicrobial as phage amplification ceases in absence of bacterial host and the equilibrium shifts towards phage inactivation. Due to differences in bacteriophage characteristics (e.g. size, surface proteins, architecture) phage delivery system requires extensive testing for every different phage. Depending on the loaded phage, difference in phage release kinetics were observed in this thesis, with considerable differences found in release kinetics of $S$. aureus phage ISP and P. aeruginosa phage LUZ19. As was discussed in Chapter 6, this was not size dependent as LUZ19 is a smaller phage than ISP, but probably due to chemical interactions related to the different content of hydrophobic amino acid in the phage proteins. This claim has to be verified with follow-up research on release of different fully characterized and sequenced bacteriophages from hydrogel systems. As most phage therapies involve the administration of phage cocktails, release rates and activity maintenance of all cocktail sub-populations inside the biomaterial should be investigated before clinical implementation. 
A distinctly different approach to infection prevention was addressed in Chapter 7. Orthopedic implants have been recognized as surfaces that are susceptible to biofilm formation due to impaired immune response at the material interface. By synthesizing anti-fouling and bactericidal PEEK surface we set out to reduce implant vulnerability to bacterial colonization. While surfaces grafted with anti-fouling poly(ethylene glycol) and contactkilling polymers containing quarternized ammonium were able to synergistically reduce the number of bacteria adhering to the surface in our in vitro experiments (Chapter 7), it did not completely prevent bacterial adhesion. Therefore, the benefit of these surfaces lies in delaying the onset of device-related infection but would most likely not be able to prevent it. The development of surfaces that would deny bacteria to adhere to the implant and simultaneously release antimicrobials would be a promising prospect that would allow killing of bacteria without the need for direct contact with the surface. Based on the experiences and results acquired in this $\mathrm{PhD}$ thesis, a proposal for further development of such surfaces has been outlined. A schematic of the envisioned surfaces can be seen in Figure 9.1. The release of antimicrobials at the initial phase of implantation is envisioned to eradicate most bacteria at the implant site, while any persisting bacteria would be repulsed by high molecular weight hydrophilic/polyelectrolyte polymer brushes. The surfaces described here could be utilized in a wide array of medical applications such as orthopedics, venous access devices or urinary catheters. 


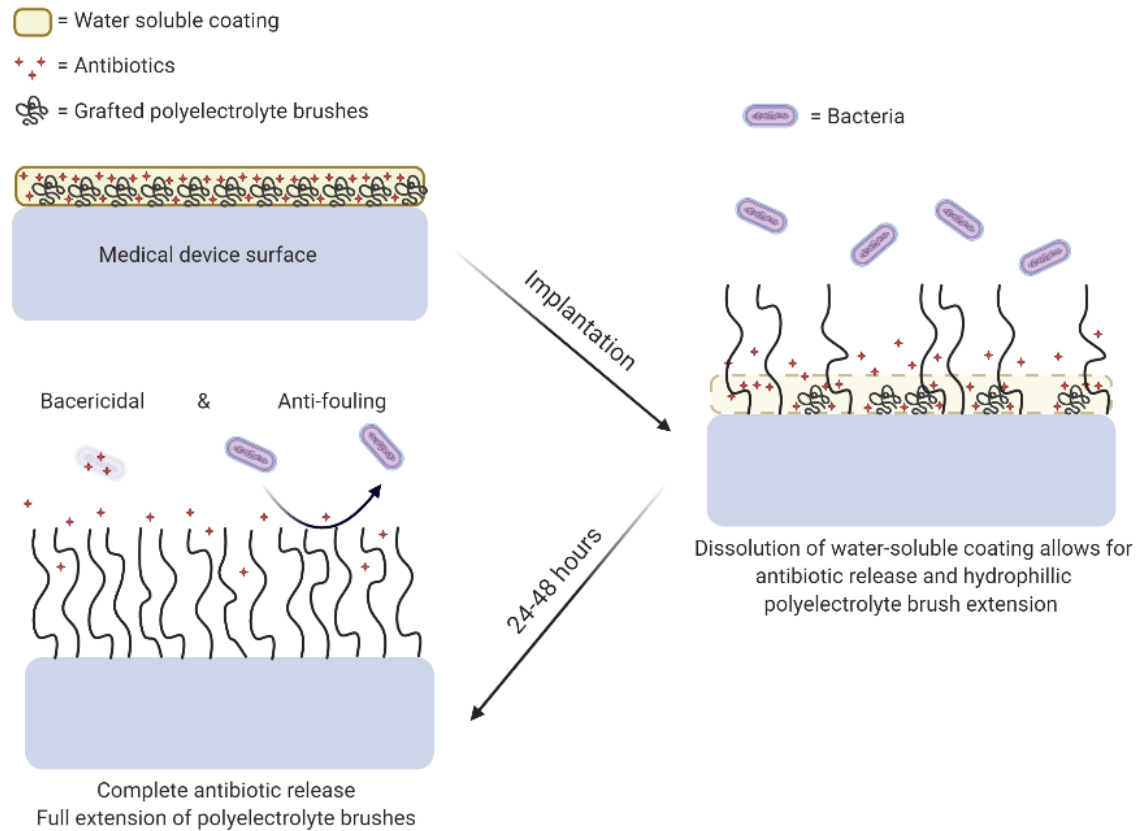

Figure 9.1 - Schematic overview of the envisioned bactericidal and antifouling coatings for future research

Generally, the preliminary in vivo data shown in this thesis highlights that generated in vitro results are not always translatable to a pre-clinical setting, underscoring the importance of thorough design of biologically relevant in vitro assays and preclinical models. A literature review by Sjollema et al. on in vitro experimental design for testing antimicrobial surfaces gives a great overview this topic [10]. For example, suitable bacterial challenge, addition of realistic quantities of fouling proteins, static/dynamic culture conditions or the implementation of clinically relevant bone defects should be considered. Deviations from clinically relevant scenarios due to practical concerns can be considered when designing pre-clinical models, but care must be taken to prevent misinterpretation of the results.

To conclude, the biomaterial approaches investigated in this thesis show potential to improve treatment options for OM. Ultimately, bone-targeting 
antibiotic delivery, bacteriophage releasing hydrogels and antimicrobial implant surfaces can all contribute to strengthening the arsenal of orthopedic surgeons in the treatment or prevention of $\mathrm{OM}$.

\section{References}

1 Tice, A. D., Hoaglund, P. A. \& Shoultz, D. A. Outcomes of osteomyelitis among patients treated with outpatient parenteral antimicrobial therapy. Am J Med 114, 723-728, doi:10.1016/s0002-9343(03)00231-6 (2003).

2 Gao, P., Nie, X., Zou, M., Shi, Y. \& Cheng, G. Recent advances in materials for extended-release antibiotic delivery system. The Journal of Antibiotics 64, 625-634, doi:10.1038/ja.2011.58 (2011).

3 Dudareva, M. et al. Short or Long Antibiotic Regimes in Orthopaedics (SOLARIO): a randomised controlled open-label non-inferiority trial of duration of systemic antibiotics in adults with orthopaedic infection treated operatively with local antibiotic therapy. Trials 20, 693, doi:10.1186/s13063-019-3832-3 (2019).

4 Farrell, K. B., Karpeisky, A., Thamm, D. H. \& Zinnen, S. Bisphosphonate conjugation for bone specific drug targeting. Bone Rep 9, 47-60, doi:10.1016/j.bonr.2018.06.007 (2018).

5 Savaridas, T., Wallace, R. J., Salter, D. M. \& Simpson, A. H. R. W. Do bisphosphonates inhibit direct fracture healing? The Bone \& Joint Journal 95-B, 1263-1268, doi:10.1302/0301-620X.95B9.31562 (2013).

6 Murphy, M. B., Hartgerink, J. D., Goepferich, A. \& Mikos, A. G. Synthesis and in Vitro Hydroxyapatite Binding of Peptides Conjugated to Calcium-Binding Moieties. Biomacromolecules 8, 2237-2243, doi:10.1021/bm070121s (2007).

7 Vedantam, R. \& Ruddlesdin, C. The fully hydroxyapatite-coated total hip implant: Clinical and roentgenographic results. The Journal of Arthroplasty 11, 534-542, doi:https://doi.org/10.1016/S0883-5403(96)80106-9 (1996).

8 Stigter, M., Bezemer, J., de Groot, K. \& Layrolle, P. Incorporation of different antibiotics into carbonated hydroxyapatite coatings on titanium implants, release and antibiotic efficacy. Journal of Controlled Release 99, 127-137, doi:https://doi.org/10.1016/j.jconrel.2004.06.011 (2004).

9 Neut, D. et al. A biodegradable gentamicin-hydroxyapatite-coating for infection prophylaxis in cementless hip prostheses. European cells \& materials 29, 42-56, doi:10.22203/ecm.v029a04 (2015).

10 Sjollema, J. et al. In vitro methods for the evaluation of antimicrobial surface designs. Acta Biomaterialia 70, 12-24, doi:https://doi.org/10.1016/j.actbio.2018.02.001 (2018). 


\section{Summary}

Bone infection is a dreaded complication in orthopedic trauma surgery which can severely delay or prevent successful patient recovery. Due to impaired vascularity at the trauma site, systemic antibiotic therapies are limited in their capacity to clear the local infection. Local antibiotics are required to improve outcome of infection treatment, with antibiotic loaded bone cements or collagen sponges being frequently utilized in clinics. However, these materials are associated with unfavorable characteristics such as inappropriate antibiotic release kinetics or non-biodegradability. This thesis reports on the development of biomaterials for implementation as local antibiotic delivery systems for treatment or prevention of orthopedic infections.

Biodegradable microspheres have been developed which can be efficiently loaded with hydrophobic antibiotics and their surfaces can be subsequently functionalized with bone targeting molecules. This approach yielded promising in vitro results leading to enhanced antibiotic delivery to mineralized substrates.

As antibiotic resistance becomes an increasingly troublesome complication in infection treatment, delivery of alternative and effective antimicrobials to infected bone tissue is becoming an urgent clinical need. Bacteriophages were embedded in a thermoresponsive hydrogel system and these hydrogels allowed for sustained antibiotic release over a 3-week period. Bacteriophage lytic activity and release kinetics could be improved by incorporation of alginate based microbeads into the hydrogels.

Biofilm formation on orthopedic devices also contribute to treatment failure of antimicrobial treatments. Due to the impaired immune response on the 
device interface, these surfaces are susceptible to bacterial adhesion and biofilm formation which can lead to persisting infections. Hydrophilic antifouling coatings and cationic bactericidal coatings were introduced in this thesis, and showed that in an in vitro set-up adhesion of viable bacteria could be synergistically reduced when both coatings were applied to poly(ether ether ketone) surfaces. These anti-fouling and antimicrobial surfaces can slow the process of device colonization, allowing prophylactic antibiotics sufficient time to prevent the development of a device-related infection.

This thesis is comprised of the development of multiple biomaterials designed for antimicrobial strategies to treat or prevent bone infection. Enhanced antibiotic delivery was achieved by designing bone targeting microspheres. Additionally, common complications such as antibiotic resistance and device-related infection have been addressed with new treatment solutions that should be further developed in pre-clinical models. 


\section{Acknowledgements}

My $\mathrm{PhD}$ studies have undoubtedly been the biggest undertaking that I have ever pursued. The four years that I spend on the various projects described in this thesis have not only improved my academic capabilities but also influenced my personal development. My PhD-journey started in the summer of 2016, when during my MSc projects a job-advert for a $\mathrm{PhD}$ position at the AO Research Institute Davos was hanging in the coffee corner of the Zuidhorst. With a brief but interesting project description and images of the beautiful Davos scenery, I realized that this was a golden opportunity for me. Thus, I was very excited to move to Davos only a few weeks later. There I would meet many great colleagues and wonderful friends that made my years in Davos fly by very quickly. Now, as I am finishing my doctoral thesis, I am grateful for every lesson learned and all the happy memories that I have made during my time at the AO Research Institute. Many people have been a great help to me throughout my time in Davos and I would like to express my thanks to all of them below.

I would like to start with thanking my promotor and supervisor Prof. Dr. David Eglin. You were available to me almost daily and were a constant help throughout my studies. In our many project meetings I could always discuss the newest results and obstacles that I was facing at that moment. I would always end these meetings with fresh ideas and increased motivation to overcome the current difficulties I was facing.

Another big thank you goes out to Prof. Dr. Dirk Grijpma for being my promotor and academic advisor. It was you who gave me the determination to apply for the $\mathrm{PhD}$ position and I always see you as the matchmaker between me and my supervisors in Davos. Even though my visits to Twente 
were usually brief and not that frequent, you could always find the time to meet with me for discussing results and for guiding me in the right direction. As our correspondence intensified in the final months of my $\mathrm{PhD}$, you gave me the extra motivation required to finish the thesis in a relatively short timeframe.

I would also like to thank Dr. Olivier Guillaume, not only for all your scientific input but also for writing the grant that funded my project! I am thankful for your guidance and sharing an office with you during the first two years of my $\mathrm{PhD}$ allowed me to constantly learn from you. Having such a determined and skilled mentor as you to get help me get familiarized with all aspects of research is something that I wish for every $\mathrm{PhD}$ student.

My thanks go out to Dr. Fintan Moriarty for his help and guidance he provided during my $\mathrm{PhD}$ studies. My knowledge in microbiology prior to my studies at ARI was non-existent, so your input and suggestions on my research were thus always great leaning experiences. By involving me in ARI's exciting bacteriophage projects, you allowed me to broaden my experiences even more, for which I am very grateful.

I would like to express gratitude to Prof. Dr. Marcel Karperien, Prof. Dr. Jai Prakash, Prof. Dr. Benjamin Nottelet and Prof. Dr. Tingting Tang for being part of my PhD committee and for assessing this thesis. Additionally, I thank Prof. Tang and his group for allowing me to visit his lab and assisting me with my in vivo investigations. My time and experiences in Shanghai were extremely useful and very pleasant due to my warm welcome I received in your group. Minqi, thank you so much for being my guide in China and sticking with me during the animal surgeries, I could not have managed to perform the surgeries without your help! 
The help of Karin at the BST secretariat in assisting me in arranging the necessary paperwork for the graduation phase of the $\mathrm{PhD}$ defense ceremony is also much appreciated. Your expertise and helpful pointers have surely helped me navigate the administrative hassles associated with finalizing a $\mathrm{PhD}$. Thank you very much!

From my first workday at ARI I was warmly invited to join the Lunch-group and since then I have never had to eat lunch alone. Gert-Jan, Simona, Yann, Marina, Ana-Maria, Dalila, Karen, Romain, Phelipe, Amir, Alejandro, Marc and so many others that have joined the Lunch-group over the years, thank you for sharing your meals, recipes and company with me. This way, lunch was always a fun and social event that provided the necessary break of the day.

I would like to thank my family for their support throughout my life. Mom and Dad, you have always made sure to look out for me and set me on the path that has led to this thesis. Lieke and Mireille, you are great sisters to have and we have a lot of fun together whenever we see each other. Thanks as well for your many visits to the Swiss Alps. Mom and Marien, I enjoyed the many hikes that we did in and around Davos and when I came to the Netherlands, I always felt like coming home. Also, your shipments of Dutch products made sure that I have never felt homesick! Dad and Lisette, we have enjoyed your visits very much and you can always drop by in Davos whenever you are on transit to Italy. We look forward to having you again soon.

Also, a big thank you to my family-in-law, Pedar, Maman, Honye, Donya and Ehsan. We have traveled together in Europe and you also showed me the most beautiful sights in Iran, for which I am forever grateful. You have 
opened my eyes to a beautiful culture and whenever you were our guests you filled our home with warmth and positive energy. I hope to see all of you more in the near future, whether it be in Iran or in Davos!

Finally, I would like to express my most heartfelt gratitude and love to my wife Reihane. I met you on my first day on the job and it was the best thing that happened to me; much more than I could ever wish for. You supported me in the difficulties that are undeniably connected to doing a $\mathrm{PhD}$ and made my life more colorful. I am excited to continue walking down the path of life with you by my side.

Thank you all!

\section{Stijn}

